
Wolf Beck

Verhaltenskodizes im Lauterkeitsrecht

Dieses Werk ist lizenziert unter einer

Creative Commons

Namensnennung - Weitergabe unter gleichen Bedingungen

4.0 International Lizenz.

(). (1) (-) 
erschienen in der Reihe der Universitätsdrucke im Universitätsverlag Göttingen 2015 


\section{Wolf Beck}

\section{Verhaltenskodizes im Lauterkeitsrecht}

Zur Frage der Unzulässigkeit von

Verstößen gegen Verhaltenskodizes und von sonstigen Verhaltensweisen im Zusammenhang mit Verhaltenskodizes nach dem UWG

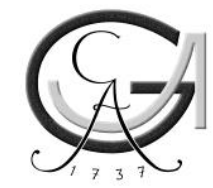

Universitätsverlag Göttingen 2015 


\section{Bibliographische Information der Deutschen Nationalbibliothek}

Die Deutsche Nationalbibliothek verzeichnet diese Publikation in der Deutschen Nationalbibliographie; detaillierte bibliographische Daten sind im Internet über $<$ http://dnb.dnb.de $>$ abrufbar.

Anschrift des Autors

Wolf Beck

E-Mail: wolf.beck@web.de

Dieses Buch ist auch als freie Onlineversion über die Homepage des Verlags sowie über den Göttinger Universitätskatalog (GUK) bei der Niedersächsischen Staats- und Universitätsbibliothek Göttingen (http://www.sub.uni-goettingen.de) erreichbar. Es gelten die Lizenzbestimmungen der Onlineversion.

Satz und Layout: Jan Kaluza

(C) 2015 Universitätsverlag Göttingen http:/ / univerlag.uni-goettingen.de ISBN: 978-3-86395-228-0 
Meiner Familie 



\section{Vorwort}

Die vorliegende Arbeit wurde im Sommersemester 2015 von der Juristischen Fakultät der Georg-August-Universität Göttingen als Dissertation angenommen. Mein Dank gilt meinem Doktorvater Herrn Professor Dr. Andreas Wiebe, LL.M für die hervorragende Betreuung der Arbeit. Herrn Professor Dr. Torsten Körber, LL.M danke ich für die Übernahme des Zweitgutachtens.

Besonderer Dank gebührt zudem meinen geliebten Eltern, die mich nicht nur während der Promotion, sondern Zeit meines Lebens in jeglicher Hinsicht bedingungslos unterstützt haben. Darüber hinaus danke ich Dir, liebste Clara, für die Inspiration, diese Arbeit anzugehen und die wunderschöne Zeit während ihrer Erstellung.

Juli 2015

Wolf Beck 



\section{Inhaltsverzeichnis}

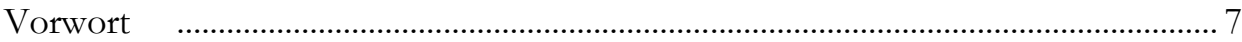

Inhaltsverzeichnis ...............................................................................................

Abkürzungsverzeichnis......................................................................................... 14

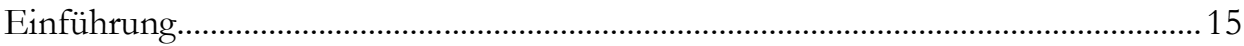

Kapitel 1 Grundsätzliches zu Verhaltenskodizes........................................................19

A. Begriffsverständnis im allgemeinen Sprachgebrauch ...................................19

B. Historische Entwicklung von Verhaltenskodizes und ihre

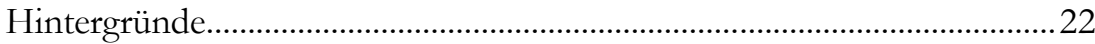

C. Kategorien von Verhaltenskodizes ……………………………………....26

I. Unternehmenseigene Verhaltenskodizes.................................................26

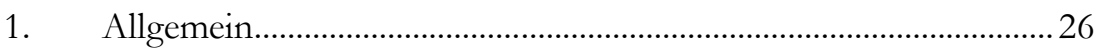

2. Beispiel: Verhaltenskodizes der Otto Gruppe..................................27

a. Verhaltenskodex Handelsware.......................................................22

b. Verhaltenskodex Dienstleistungen und Nicht-Handelsware ...29

II. Gemeinsame Verhaltenskodizes unabhängiger Unternehmen............29

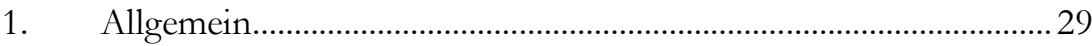

2. Beispiel: Responsible Care Programme des VCI und des VCH ... 30

III. Verhaltenskodizes von Unternehmen unter Beteiligung unabhängiger, nichtwirtschaftlicher Dritter............................................... 33

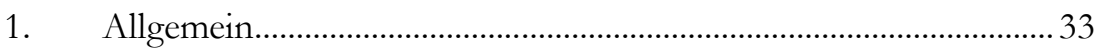

2. Beispiel: UN Global Compact ........................................................... 33

D. Bindungswirkung von Verhaltenskodizes....................................................35

Kapitel 2 Die Bedeutung von Verhaltenskodizes im UWG....................................... 39

A. Unter dem UWG 1909 und dem UWG 2004 _.................................................39

B. Unter dem UWG 2008............................................................................... 41

I. Europäischer Ursprung der Regelungen zu Verhaltenskodizes im

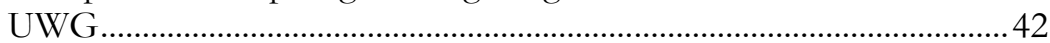

1. Grundsätzliches zur UGP-Richtlinie .................................................. 42

a. Historie, Anwendungsbereich und Ziel....................................... 42

b. Aufbau und Systematik .............................................................. 44 
2. Verhaltenskodizes in der UGP-Richtlinie ...........................................46

a. Bedeutung der Selbstregulierung in der Gesetzgebungshistorie der UGP-Richtlinie.............................................................. 46

b. Regelungen zur Verhaltenskodizes in der UGP-Richtlinie........ 48

II. Regelungen zu Verhaltenskodizes im UWG 2008.................................50

Kapitel 3 Der Begriff des Verhaltenskodexes i. S. d. UWG.......................................53

A. Freiwilligkeit der Verpflichtung ...................................................................54

B. Beschränkung auf Verpflichtungen von Unternehmern .............................55

C. Gegenstand der Verpflichtung.....................................................................58

D. Notwendiger Grad der Verpflichtung ..........................................................61

E. Notwendiger Inhalt von Verhaltenskodizes ...................................................62

F. Kartellrechtliche Problematik von Verhaltenskodizes i. S. d.

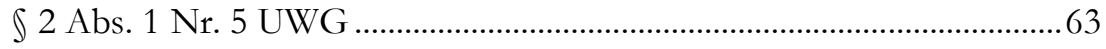

I. Verhaltenskodizes als unzulässige Kartellvereinbarung..........................64

II. Freistellung gemäß $\$ 2$ GWB beziehungsweise Art. 101 Abs. 3 AEUV.

III. Anerkennung von Verhaltenskodizes als Wettbewerbsregel

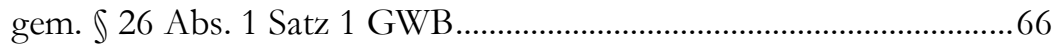

G. Zusammenfassung .................................................................................. 67

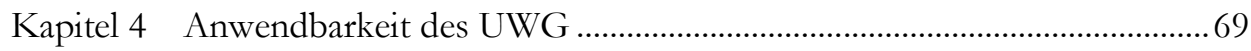

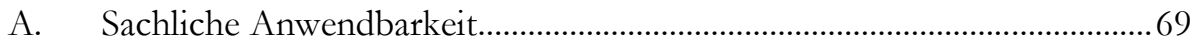

I. Grundsätzliches zur geschäftlichen Handlung ........................................70

II. Voraussetzungen der geschäftlichen Handlung ......................................72

1. Verhalten einer Person zugunsten des eigenen oder eines fremden Unternehmens ...................................................................... 72

2. Objektiver Zusammenhang mit der Förderung des Absatzes oder des Bezuges von Waren oder Dienstleistungen oder mit dem Abschluss oder der Durchführung eines Vertrages über Waren und Dienstleistungen .................................................................76

a. Objektiver Zusammenhang im b2c-Bereich............................... 77

b. Objektiver Zusammenhang im b2b-Bereich............................... 80

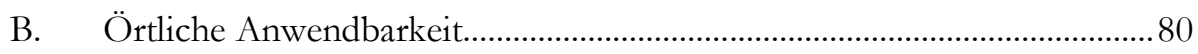

C. Persönliche Anwendbarkeit........................................................................ 84 
Kapitel 5 Spezialregelungen zu geschäftlichen Handlungen im

Zusammenhang mit Verhaltenskodizes .87

A. Geschäftliche Handlungen im Zusammenhang mit

Verhaltenskodizes im Anhang zu $\int 3$ Abs. 3 UWG. 88

I. Die Grundregelung in $\ 3$ Abs. 3 UWG............................................. 88

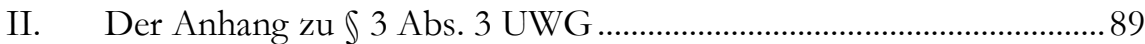

1. Unwahre Angabe zu den Unterzeichnern eines

Verhaltenskodexes zu gehören, Nr. 1 des Anhangs zu \ 3

Abs. 3 UWG

2. Unwahre Angabe über die Billigung eines Verhaltenskodexes, Nr. 3 des Anhangs zu \ 3 Abs. 3 UWG .......................................... 94

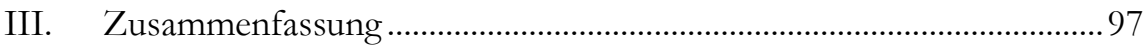

B. Irreführende Angabe über die Einhaltung eines Verhaltenskodex, $\ 5$

Abs. 1 Satz 2 Nr. 6 UWG ......................................................................... 98

I. Systematik der Norm, Prüfungszusammenhang mit

$\int 3$ Abs. 1 UWG.

II. Die Voraussetzungen der Irreführung aus

$\int 5$ Abs. 1 Satz 2 UWG

III. Die Voraussetzungen der Nr. 6 des $\int 5$ Abs. 1 Satz 2 UWG .............102

1. Verbindliche Verpflichtung auf Verhaltenskodex .........................102

2. Hinweis auf Bindung an Verhaltenskodex......................................105

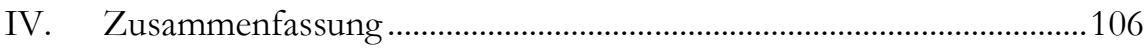

Kapitel 6 Bewertung geschäftlicher Handlungen im Zusammenhang mit Verhaltenskodizes nach den sonstigen Regelungen des UWG zur Unlauterkeit.

A. Unlauterkeit von Verhaltensweisen im Zusammenhang mit

Verhaltenskodizes gemäß \ 4 UWG

I. Hinweis auf Verhaltenskodex als Beeinträchtigung der

Entscheidungsfreiheit durch unangemessene, unsachliche

Einflussnahme, \ 4 Nr. 13 . Alt. UWG

1. Rechtsprechung zur gefühlsbetonten Werbung...

a. Frühere Rechtsprechung zur gefühlsbetonten Werbung........111

b. Auslöser der Rechtsprechungsänderung zur gefühlsbetonten Werbung.

c. Aktuelle Rechtsprechung zur gefühlsbetonten Werbung.......114

2. Verbliebener Anwendungsbereich des $\int 4$ Nr. 1 3. Alt. UWG....115

3. Zusammenfassung..... 
II. Gezielte Behinderung von Mitbewerbern durch Verhaltenskodizes, \4 Nr. 10 UWG

III. Verstoß gegen eine gesetzliche Vorschrift, \ 4 Nr. 11 UWG ............118

1. Richtlinienkonforme Auslegung ....................................................118

2. Begriff der gesetzlichen Vorschrift................................................119

3. Zusammenfassung .................................................................... 120

B. Irreführung durch aktives Handeln, \5 UWG ......................................121

I. Irreführung durch unwahre Angaben aus Nr. 1 und Nr. 3 des Anhangs zu \3 Abs. 3 UWG im b2b-Bereich .....................................122

1. Grundsätzliches zur Irreführung bei unwahren Angaben .............122

2. Unwahre Angabe, zu den Unterzeichnern eines

Verhaltenskodexes zu gehören.....................................................124

3. Unwahre Angabe über Billigung eines Verhaltenskodex...............127

II. Irreführung trotz objektiver Richtigkeit der Angaben aus Nr. 1 und Nr. 3 des Anhangs zu \3 Abs. 3 UWG....

1. Irreführung trotz tatsächlicher Billigung eines Verhaltenskodexes durch eine öffentliche oder eine andere Stelle

a. Irreführung wegen Fehlens der Voraussetzungen der Billigung.....

b. Irreführung aufgrund fehlender Neutralität der billigenden Stelle.

c. Irreführung aufgrund der Bezeichnung der billigenden Stelle.

2. Irreführung trotz tatsächlicher Unterzeichnung eines Verhaltenskodexes

III. Irreführung durch weitere Angaben im Zusammenhang mit Verhaltenskodizes

1. Irreführung durch Angaben über den Inhalt eines

Verhaltenskodexes

2. Irreführung durch Angaben zur Überprüfung der Einhaltung eines Verhaltenskodexes ....

IV. Irreführung aufgrund einer Verwechslungsgefahr.............................137

1. Beschränkung der betroffenen geschäftlichen Handlungen ........137

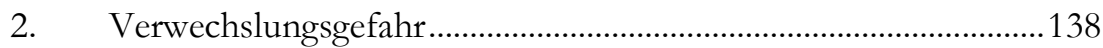

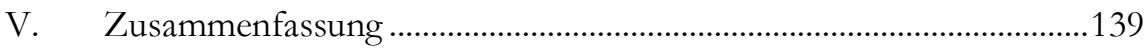

C. Irreführung durch Unterlassen bei geschäftlichen Handlungen im Zusammenhang mit Verhaltenskodizes, \5a UWG

I. Geschäftliche Handlungen gegenüber Unternehmern. 
1. Aufklärungspflicht über die bestehende oder nicht (mehr) bestehende Bindung an einen Verhaltenskodex

2. Aufklärungspflicht über den Verstoß gegen einen

Verhaltenskodex 146

II. Geschäftliche Handlungen gegenüber Verbrauchern ........................... 146

1. Wesentlichkeit der Informationen.................................................. 148

a. Wesentlichkeit nach $\int 5 a$ Abs. 3 UWG ...................................148

aa. Erfasste geschäftliche Handlungen ......................................150

bb. Informationen im Zusammenhang mit Verhaltenskodizes als wesentliche Information gemäß $₫ 5 \mathrm{a}$ Abs. 3 UWG

b. Wesentlichkeit nach $\int 5 a$ Abs. 4 UWG ...................................156

c. Wesentlichkeit nach $\int 5$ a Abs. 2 UWG .....................................157

2. Vorenthalten einer wesentlichen Information................................160

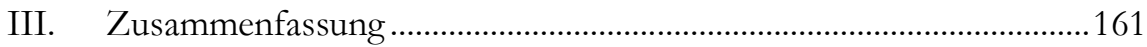

D. Unlauterkeit beziehungsweise Unzulässigkeit von Verstößen gegen Verhaltenskodizes gemäß der Generalklauseln aus $\ 3$ Abs. 1 und Abs. 2 Satz 1 UWG

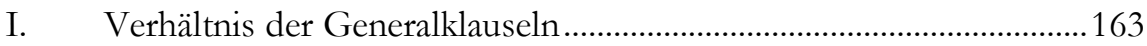

II. Bedeutung von Verstößen gegen Verhaltenskodizes im Rahmen der Generalklauseln ............................................................................... 166

1. Ansatz der Rechtsprechung: Indizwirkung für Unlauterkeit .......166

2. Bewertung der Rechtsprechung, insbesondere im Hinblick auf ihre Vereinbarkeit mit der UGP-Richtlinie ............................... 169

a. Beschränkung auf Branchenregelungen ................................... 169

b. Kein Automatismus zwischen dem Verstoß einer geschäftlichen Handlung gegen einen Verhaltenskodex und deren Unlauterkeit.

c. Ermittlungshilfe zur Bestimmung der fachlichen Sorgfalt / Indizwirkung von Verstößen gegen Verhaltenskodizes.

III. Zusammenfassung ............................................................................. 175

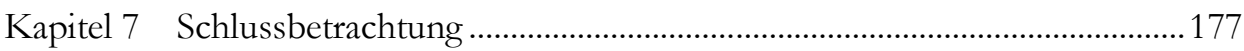

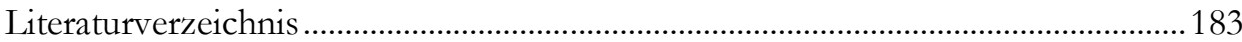




\section{Abkürzungsverzeichnis}

$\begin{array}{ll}\text { AEUV } & \text { Vertrag über die Arbeitsweise der Europäischen } \\ & \text { Union } \\ \text { BeckRS } & \text { Beck Rechtsprechung } \\ \text { bspw. } & \text { beispielsweise } \\ \text { BVDVA } & \text { Bundesverband Deutscher Versandapotheken } \\ \text { bzgl. } & \text { bezüglich } \\ \text { bzw. } & \text { beziehungsweise } \\ \text { ca. } & \text { circa } \\ \text { CSR } & \text { Corporate Social Responsibility } \\ \text { DPMA } & \text { Deutsches Marken- und Patentamt } \\ \text { E-Commerce-Richtlinie } & \text { Richtlinie über den elektronischen Geschäftsverkehr } \\ \text { Einf. } & \text { Einführung } \\ \text { etc. } & \text { et cetera } \\ \text { ETI } & \text { Ethic Trade Initiative } \\ \text { FAQ } & \text { Frequently Asked Questions } \\ \text { ggü. } & \text { gegenüber } \\ \text { MarkenG } & \text { Markengesetz } \\ \text { PreisKlG } & \text { Preisklauselgesetz } \\ \text { sog. } & \text { sogenannt } \\ \text { TMG } & \text { Telemediengesetz } \\ \text { u.a. } & \text { unter anderem } \\ \text { UGP-Richtlinie } & \text { Richtlinie über unlautere Geschäftspraktiken } \\ \text { USA } & \text { United States of America, Vereinigte Staaten von } \\ \text { UVP } & \text { Amerika } \\ \text { VCH } & \text { Unverbindliche Preisempfehlung } \\ \text { VCI } & \text { Verband Chemiehandel } \\ \text { WWF } & \text { Verband der Chemischen Industrie } \\ & \text { World Wildlife Fund } \\ & \end{array}$

Im Übrigen wird verwiesen auf Kirchner, Hildebert, Abkürzungsverzeichnis der Rechtssprache, 7. völlig neu bearbeitete und erweiterte Auflage, 2013. 


\section{Einführung}

Elemente der Selbstregulierung waren dem deutschen Lauterkeitsrecht unter dem UWG 1909 sowie dem UWG 2004 ebenso fremd wie der Begriff des Verhaltenskodexes selbst. ${ }^{1}$ Zwar waren von Privaten geschaffene Wettbewerbsregeln beziehungsweise Wettbewerbsrichtlinien Gegenstand der Rechtsprechung sowie der Literatur. Jedoch spielte die Selbstregulierung als solche im deutschen Lauterkeitsrecht im Gegensatz zu dem anderer Mitgliedstaaten der Europäischen Union wie beispielsweise dem Vereinigten Königreich, den Niederlanden oder Italien² kaum eine Rolle. Entsprechend war eine Sanktionierung von Verstößen gegen Verhaltenskodizes nur mit Hilfe von „rechtlich kreativen“ Lösungen auf Basis der lauterkeitsrechtlichen Generalklausel möglich. ${ }^{3}$

Dies hat sich mit dem am 22.12.2008 vom Deutschen Bundestag beschlossenen und am 30.12.2008 in Kraft getretenen ${ }^{4}$ „Ersten Gesetz zur Änderung des Gesetzes gegen den unlauteren Wettbewerb" zur Umsetzung der am 11.05.2005 erlassenen Richtlinie 2005/29/EG über unlautere Geschäftspraktiken (im Folgenden: UGP-

\footnotetext{
${ }^{1}$ Begründung der Bundesregierung für den Gesetzentwurf zur Änderung des UWG vom 20.08.2008, BT.-Drs. 16/10145, S. 12.

2 Schulze/Schulte-Nölke, S. 8 ff.

${ }^{3}$ Kocher, Rechtliche Instrumente, insbesondere zur Durchsetzung und Umsetzung von Codes of Conduct im deutschen Recht, in Köpke/Röhr, S. 179 f.

${ }^{4}$ Am Tag nach seiner Verkündung im BGBl., Jahrgang 2008, Teil I, Nr. 64, 2949 ff.
} 
Richtlinie) erheblich geändert. So enthält das UWG 2008 entsprechend der Vorgaben der UGP-Richtlinie nicht nur die Legaldefinition eines Verhaltenskodexes ${ }^{5}$, sondern neben Regelungen zur Unzulässigkeit von irreführenden Angaben im Zusammenhang mit Verhaltenskodizes ${ }^{6}$ insbesondere auch eine Regelung zur Irreführung von Verstößen gegen Verhaltenskodizes. ${ }^{7}$ Insbesondere die Regelung über die Irreführung von Verstößen gegen Verhaltenskodizes wurde in der Literatur skeptisch aufgenommen. So wurde die Frage aufgeworfen, ob das Lauterkeitsrecht nunmehr zum Motor für die Durchsetzung vertraglicher Verpflichtungen werde. ${ }^{8}$ Diese Skepsis gründet auch auf der erheblichen Verbreitung von Verhaltenskodizes und dem damit einhergehenden potenziellen Wirkungsbereich der Norm. Denn Verhaltenskodizes haben bei den Unternehmen der westlichen Welt mittlerweile eine solche Verbreitung erfahren, dass ihr Vorliegen zumindest bei international tätigen Unternehmen als Standard bezeichnet werden kann. ${ }^{9}$ So dürfte es heutzutage der sprichwörtlichen Suche nach der Nadel im Heuhaufen gleichkommen, ein in diesen Ländern börsennotiertes Unternehmen ohne Verhaltenskodex zu finden. Zudem haben mittlerweile auch viele Universitäten ${ }^{10}$, NROen ${ }^{11}$ und sogar die Politik ${ }^{12}$ Verhaltenskodizes unterzeichnet.

Aufgrund des auf geschäftliche Handlungen begrenzten Anwendungsbereiches des UWG stehen jedoch nur die Verhaltenskodizes von Unternehmen im Blickpunkt dieser Arbeit. Dabei beschränkt sich diese nicht auf die Frage, unter welchen Voraussetzungen der Verstoß gegen einen Verhaltenskodex eine unzulässige geschäftliche Handlung darstellt. Sie untersucht vielmehr vollumfänglich, welche Verhaltensweisen im Zusammenhang mit Verhaltenskodizes nach dem UWG unzulässig sind. Die Arbeit beginnt in Kapitel 1 zunächst mit dem Verständnis des Begriffs des Verhaltenskodexes im allgemeinen Sprachgebrauch. Im Anschluss hieran stellt sie die historische Entwicklung von Verhaltenskodizes dar sowie die unterschiedlichen Kategorien von Verhaltenskodizes anhand von Beispielen vor.

$5 \int 2$ Abs. 1 Nr. 5 UWG.

${ }^{6}$ Nr. 1 und Nr. 3 des Anhangs zu $₫ 3$ Abs. 3 UWG i. V. m. $\int 3$ Abs. 3 UWG.

$7 \int 5$ Abs. 1 Satz 2 Nr. 6 UWG.

8 Dreyer, WRP 2007, 1294, 1295 die bereits in der Unterzeile der Überschrift ihres Artikels die Frage stellt, ob dass Wettbewerbsrecht mit dem „Ersten Gesetz, zur Änderung des Gesetzes gegen den unlauteren Wettbewerb“ „zum Motor für die Durchsetzung vertraglicher Verpflichtungen wird".

${ }^{9}$ So haben sich bspw. sämtliche 30 im Deutschen Aktien Index gelisteten Unternehmen Verhaltenskodizes abgegeben.

${ }^{10}$ Vgl. „,Verbaltenskodex und Ethikregeln gegen Korruption“ der Georg-August Universität Göttingen, , abrufbar unter http://www.uni-goettingen.de/de/51133.html, zuletzt abgerufen am 08.08.2013.

11 Vgl. Verhaltenskodex der VENRO (Bundesverband entwicklungspolitischer Nichtregierungsorganisationen), abrufbar unter http://venro.org/venro/venro-kodizes/, zuletzt abgerufen am 08.08.2013.

12 Vgl. Verhaltenskodex der Abgeordneten des Europäischen Parlamentes, abrufbar unter http://www.europarl.europa.eu/aboutparliament/de/0081ddfaa4/Abgeordnete.html, zuletzt abgerufen am 08.08.2013. 
Den Abschluss von Kapitel 1 bildet eine Untersuchung der Bindungswirkung von Verhaltenskodizes. Kapitel 2 zeigt die Entwicklung der lauterkeitsrechtlichen Gesetzeslage zu Verhaltenskodizes, erläutert den europarechtlichen Hintergrund der benannten Gesetzesänderung und stellt die mit dieser in das UWG aufgenommen Regelungen zu Verhaltenskodizes vor. Kapitel 3 untersucht, welche Regelwerke als Verhaltenskodex i. S. d. $\int 5$ Abs. 1 Nr. 5 UWG anzuerkennen sind. Kapitel 4 stellt die grundsätzlichen Voraussetzungen der Anwendbarkeit des UWG unter besonderer Beachtung der Besonderheiten von Verhaltensweisen im Zusammenhang mit Verhaltenskodizes dar. In Kapitel 5 erfolgt die Untersuchung der Spezialregelungen zu Verhaltenskodizes aus den Nr. 1 und 3 des Anhangs zu \ 3 Abs. 3 UWG und $\int 5$ Abs. 1 Satz 2 Nr. 6 UWG. In Kapitel 6 wird dargestellt, wie geschäftliche Handlungen im Zusammenhang mit Verhaltenskodizes von den sonstigen Regelungen des UWG erfasst werden. Kapitel 7 behandelt die vom UWG für unzulässige geschäftliche Handlungen im Zusammenhang mit Verhaltenskodizes vorgesehen Sanktionen. Die Schlussbetrachtung in Kapitel 8 fasst die Ergebnisse der Arbeit zusammen. 



\section{Kapitel 1}

\section{Grundsätzliches zu Verhaltenskodizes}

\section{A. Begriffsverständnis im allgemeinen Sprachgebrauch}

Bevor die Bedeutung von Verhaltenskodizes im deutschen Lauterkeitsrecht untersucht wird, soll zunächst das Verständnis des Begriffs im allgemeinen Sprachgebrauch festgestellt werden. Dies insbesondere aufgrund der Tatsache, dass der allgemeine Sprachgebrauch Rückschlüsse auf das Verständnis des Durchschnittsbürgers zulässt. Denn dieses Verständnis des Durchschnittsverbrauchers spielt im deutschen wie im europäischen Lauterkeitsrecht eine entscheidende Rolle. So erklärt die UGP-Richtlinie „,den Durchschnittsverbraucher, der angemessenen gut unterrichtet und angemessen aufmerksam und kritisch ist", zum Maßstab ihrer Regelungen und Bewertungen. ${ }^{13}$ Hierbei bezieht sich die UGP-Richtlinie ausdrücklich ${ }^{14}$ auf die vom EuGH bei der Auslegung des Gemeinschaftsrechts heranzuziehende fiktive Person des „durchschnittlich informierten, aufmerksamen und verständigen Durchschnittsverbrau-

13 UGP-Richtlinie, Erwägungsgrund 18.

14 UGP-Richtlinie, Erwägungsgrund 18. 


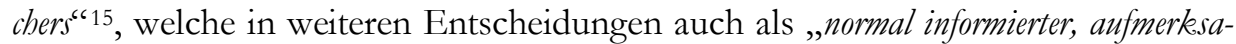
mer und verständiger Durchschnittsverbraucher"16 und „normal informierter und angemessen aufmerksamer und verständiger Durchschnittsverbraucher" $" 17$ bezeichnet wird. Dieses gemeinschaftsrechtliche Verbraucherleitbild wurde mittlerweile vom BGH ${ }^{18}$ auch für Deutschland übernommen und gilt für das gesamte Lauterkeitsrecht. ${ }^{19}$

Einen ersten Ansatz für das Verständnis von zusammengesetzten Begriffen wie dem des Verhaltenskodexes bietet das Verständnis der Einzelbegriffe. Dabei ist der Begriff des Kodexes in der heutigen Zeit weniger als Gesetzessammlung ${ }^{20}$ $\mathrm{zu}$ verstehen, sondern vielmehr als „Sammlung der Normen, Regeln eines Sacbbereiches" ${ }^{\text {" }} 1$ beziehungsweise "die ungeschriebenen Regeln des Verbaltens, des Handelns, an denen sich eine (gesellschaftliche) Gruppe orientiert"22. Ein Verhaltenskodex ist dementsprechend die Sammlung von Verhaltensregeln, an die sich eine bestimmte Person oder Gruppe freiwillig gebunden hat. Das Merkmal der Freiwilligkeit ergibt sich dabei aus dem Begriff der ungeschriebenen Regeln. Dieser ist in diesem Zusammenhang nicht wörtlich zu verstehen, sondern dahingehend, dass die Regelungen nicht (durch einen Gesetzgeber) vorgeschrieben sind.

Einen weiteren Ansatzpunkt stellen bestehende Begriffsbestimmungen dar. Bei diesen muss jedoch ein besonderes Augenmerk auf den Hintergrund der bestimmenden Person und den thematischen Zusammenhang gelegt werden. Die Europäische Kommission definiert einen Verhaltenskodex im Grünbuch CSR ${ }^{23}$ als ,formelle Erklärung zu den Werten und Aktivitäten eines Unternehmens, vielfach auch dessen Zulieferer." Ein solcher Verhaltenskodex gibt nach dem Verständnis der Europäischen Kommission „Mindeststandards vor in Verbindung mit einer Verpflichtung des Unternehmens, diese Standards einzubalten und auch von Vertragsunternebmen, Nachunternehmern, Zulieferern und Liženznehmern deren Einhaltung zu verlangen. \{...\}.“"24 Auffällig bei dieser Definition ist zunächst die Beschränkung auf Erklärungen von Unter-

${ }^{15}$ EuGH, Urteil vom 16.07.1998 - C-210/94 (Gut Springenheide) in GRUR Int 1998, S. 795, 797, Tz 31, 37.

16 EuGH, Urteil vom 06.05.2003 - C-104/01 (Libertel) in GRUR 2003, S. 604, 607, Tz 46.

${ }^{17}$ EuGH, Urteil vom 16.09.2004 - C-329/02 (SAT.1) in GRUR Int 2005, S. 44, 45, Tz 24.

${ }^{18}$ Beispiel für die Rechtssprechungsänderung: Einerseits BGH, Urteil vom 20.03.1986 - I ZR 228/83 (KG Berlin) (umgekehrte Versteigerung I) in GRUR 1986, S. 622 f. und andererseits BGH, Urteil vom 13.03.2003 - I ZR 212/00 (OLG Düsseldorf) (umgekehrte Versteigerung II) in GRUR 2003, S. $465 \mathrm{f}$.

${ }^{19}$ Bis zu seiner Rechtsprechungsänderung (Fn. 18) legte die Rspr. bei der Anwendung des UWG das Leitbild des ,flüchtigen" bzw. ,unkritischen" Verbrauchers zugrunde (vgl. unter anderem BGH, Urteil vom 29.04.1982 - I ZR 111/80 (OLG Stuttgart) (Elsässer Nudeln) in GRUR 1982, S. $564,566$.

${ }^{20}$ Entsprechend seiner Bedeutung im römischen Recht.

${ }^{21}$ http://www.duden.de/rechtschreibung/Kodex, Nr. 3 der Bedeutungsübersicht.

22 http://www.duden.de/rechtschreibung/Kodex, Nr. 4 der Bedeutungsübersicht, zuletzt abgerufen am 15.07.2013.

23 Grünbuch CSR, Anhang Begriffsbestimmungen, S. 28.

${ }^{24}$ Grünbuch CSR, Anhang Begriffsbestimmungen, S. 28. 
nehmen. Diese erklärt sich jedoch augenscheinlich mit dem mit dem Grünbuch CSR behandelten Themenbereich der sozialen Verantwortung von Unternehmen. Darüber hinaus stellt diese Definition der Europäischen Kommission zur Form von Verhaltenskodizes fest, dass es sich um formelle Erklärungen handelt. Zum Inhalt von Verhaltenskodizes kann ihr entnommen werden, dass Verhaltenskodizes Angaben zu Werten und Aktivitäten von Unternehmen enthalten und Mindeststandards vorgeben. Damit entspricht diese Definition der Europäischen Kommission im Grünbuch CSR im Wesentlichen der vielfach ${ }^{25}$ angeführten Definition von Verhaltenskodizes durch Scherrer und Greven als „schriftlich niedergelegte Richtlinien, die als Grundlage für das Verhalten transnationaler Konzerne gegenüber den staatlichen Bebörden, ibren Belegschaften, Subunternehmen und Zulieferern und der Umwelt im jeweiligen Gastland dienen." 26

Darüber hinaus ergeben sich Hinweise auf das Verständnis von Begriffen im allgemeinen Sprachgebrauch durch die Heranziehung von synonym verwendeten Begriffen. Als Synonym für den Begriff des Verhaltenskodexes wird in Deutschland der Begriff der (freiwilligen) Selbstverpflichtung verwendet. ${ }^{27}$ Dieser ist als zusammengesetzter Begriff als selbstauferlegte Verpflichtung zu verstehen. Der teilweise vorangestellte Begriff der Freiwilligkeit ist daher nur als Verdeutlichung zu betrachten, da sich die Freiwilligkeit bereits aus der Tatsache ergibt, dass die Verpflichtung selbstauferlegt ist. Ein solches Begriffsverständnis legt auch das BMU zugrunde. Dies ergibt sich aus der Definition der Selbstverpflichtungen durch das BMU als ,rechtlich unverbindliche Erklärungen von Unternehmen und Verbänden, in denen umweltpolitische Ziele festgelegt werden, die in einem vorgegebenen Zeitraum durch eigenverantwortliches Handeln der Wirtschaft realisiert werden sollen" bestimmt. ${ }^{28}$ Dabei wird durch den Verweis auf die rechtliche Unverbindlichkeit wie beim Kodex herausgestellt, dass entsprechende Verhaltensweisen nicht durch den Gesetzgeber vorgeschrieben sind. Die abermalige Beschränkung auf Erklärungen von Unternehmen und Verbänden ist dabei wie die Beschränkung auf umweltpolitische Ziele als Inhalt von Selbstverpflichtungen dem Handlungsbereich des BMU geschuldet.

Entsprechend dieser Wortbedeutungen und der bestehenden Definitionen sowohl des Begriffs des Verhaltenskodexes selbst als auch seines Synonyms der Selbstverpflichtung kann festgestellt werden, dass Verhaltenskodizes im allgemeinen Sprachgebrauch als formelle, aber selbstauferlegte Verpflichtungen auf bestimmte Verhaltensweisen verstanden werden. Trotz der entsprechenden Be-

\footnotetext{
${ }^{25}$ So unter anderem von Biedermann, S. 13 und Röns, S. 1.

${ }^{26}$ Scherrer/Greven, Instrumente zur Durchsetzung fundamentaler Arbeiterrechte, in: Blickle, Dietzfelbinger, Hütter, Scherer, Globalisierung und Sozialstandards, S. 163.

${ }^{27}$ Köpke/Röhr, S. 10.

28 http://www.bmu.de/wirtschaft_und_umwelt/selbstverpflichtungen/doc/36514.php, zuletzt abgerufen am 15.07.2013.
} 
schränkungen in den angeführten Definitionen können Verhaltenskodizes nach ihrem Verständnis im allgemeinen Sprachgebrauch jedoch nicht nur von Unternehmen, sondern auch von der Politik ${ }^{29}$, von Universitäten ${ }^{30}$ und von NROen ${ }^{31}$ abgegeben werden.32 Ansatzpunkte zum konkreten Gegenstand der mit einem Verhaltenskodex eingegangen Verpflichtungen können dem Begriff des Verhaltenskodexes selbst zwar nicht entnommen werden, jedoch verbindet der allgemeine Sprachgebrauch Verhaltenskodizes grundsätzlich mit einer über die gesetzlichen Anforderungen hinausgehenden Verpflichtung zu korrektem und anständigen Verhalten. Dies betrifft bei Unternehmen beispielsweise den Umgang mit Arbeitskräften, den Umweltschutz sowie den Verzicht auf Korruption. Bei der Politik, bei Universitäten oder NROen betrifft es dahingegen hauptsächlich die Transparenz von Entscheidungsabläufen sowie die Vermeidung von Interessenskonflikten und Korruption. 33

\section{B. Historische Entwicklung von Verhaltenskodizes und ihre Hintergründe}

Entsprechende freiwillige Verpflichtungen von Unternehmen verbreiteten sich in Deutschland erstmals Anfang der 1970er Jahre im Bereich des Umweltschutz. ${ }^{34}$ Ausgehend von der Selbstverpflichtung zur Kennzeichnung enzymhaltiger Waschmittel im Jahr 1971 verpflichtete sich die Wirtschaft in der Folge zur Unterlassung einer Vielzahl von umweltschädlichen Verhaltensweisen. ${ }^{35}$ Bis 1995 entstanden in Deutschland ca. $80^{36}$ Verhaltenskodizes im Umweltbereich, so unter anderem zu dem Umgang mit umweltschädlichen oder wiederverwertbaren Abfällen ${ }^{37}$, über den Verzicht auf umweltschädliche Inhaltsstoffe ${ }^{38}$, zu dem Verzicht der Einleitung bestimmter Stoffe in das Wassersystem und zur Reduzierung von $\mathrm{CO}_{2}$-Emissionen. ${ }^{39}$ Formell erfolgten sämtliche dieser Verhaltenskodizes mit einer Ausnahme im Rahmen von einseitigen Erklärungen der Wirtschaft ohne formelle

\footnotetext{
${ }^{29}$ vgl. Verhaltenskodex der Abgeordneten des Europäischen Parlamentes.

${ }^{30}$ vgl. „Verbaltenskodex und Ethikregeln gegen Korruption“ der Georg-August Universität Göttingen

${ }^{31}$ vgl. Verhaltenskodex der VENRO (Bundesverband entwicklungspolitischer Nichtregierungsorganisationen).

32 Biedermann, S. 16 f.

33 Vgl. Fn. 29, 30 \& 31.

${ }^{34}$ Frenz, Einf., S. 1.

35 Vgl. Fn. 34.

$36 \mathrm{Im}$ Gebiet der Europäischen Union sogar über 350, Für eine Übersicht über die Staaten der Europäischen Union im Jahre 1995 vgl. KOM (96) 561, Anlage.

37 Wie bspw. Batterien, alten Fahrzeugen, Papier.

38 Asbest, FCKW.

${ }^{39} \mathrm{KOM}$ (96) 561 endg., Anlage.
} 
Beteiligung der Behörden. ${ }^{40}$ Die Behörden wirkten jedoch informell bei deren Erstellung mit und erkannten bestimmte Verhaltenskodizes durch Pressemitteilungen oder -konferenzen der beteiligten Behörden informell an. ${ }^{41}$ Hintergrund dieser Selbstverpflichtungen im Umweltbereich war neben dem gestiegenen Verantwortungsbewusstsein der beteiligten Unternehmen und Wirtschaftskreise zumeist entweder ein mehr oder weniger sanfter Druck der Politik auf die Unternehmen, andernfalls gesetzliche Regelungen zu treffen ${ }^{42}$ oder die Erwartung der Wirtschaft, dass der Staat im Gegenzug auf eine gesetzliche Regulierung verzichtet. ${ }^{43}$ So steht die Verschärfung der $\mathrm{CO}_{2}$-Minderungsziele aus der Klimaschutzerklärung der deutschen Wirtschaft vom 09.11.2000 gegenüber ihrer Erklärung von 1996 sicherlich auch im Zusammenhang mit der zu diesem Zeitpunkt diskutierten Einführung eines verpflichtenden Emissionshandelssystems in Europa. Ein solcher Verzicht auf eine staatliche Regulierung Zug-um-Zug gegen die freiwillige Verpflichtung von Unternehmen ist jedoch nicht nur für die Unternehmen vorteilhaft, sondern auch für die Politik und die Gesellschaft. Denn wenn die Unternehmen diese Selbstverpflichtungen einhalten, können die mit diesen verfolgten Umweltzielen schneller, effizienter und flexibler erreicht werden, als dies bei einer gesetzlichen Regelung möglich ist. ${ }^{4}$

Außerhalb von Deutschland gelten die Sullivan-Principles aus dem Jahr 197745, welche — einhergehend mit der US-amerikanischen Tradition bei der Benennung von Rechtsakten — nach ihrem Schöpfer benannt sind, als wichtiger historischer Ausgangspunkt für die Entwicklung von Verhaltenskodizes. ${ }^{46}$ Leon H. Sullivan, Pastor und als Mitglied des Aufsichtsrates von General Motors das erste afro-amerikanische Mitglied eines solchen Gremiums in einem USamerikanischen Großkonzern, entwickelte diesen Verhaltenskodex für in Südafrika tätige Unternehmen, um das dort herrschende Apartheitsregime unter Druck zu setzen. Die Sullivan-Principles enthielten unter anderem die Verpflichtung zur

\footnotetext{
40 Vgl. Fn. 39.

${ }^{41}$ Vgl. Fn. 39.

42 Vgl. Fn. 39.

43 http://www.bmu.de/wirtschaft_und_umwelt/selbstverpflichtungen/doc/36514.php, zuletzt abgerufen am 15.07.2013.

${ }^{44}$ http://www.bmu.de/wirtschaft_und_umwelt/selbstverpflichtungen/doc/36514.php, zuletzt abgerufen am 15.07.2013.
}

${ }^{45}$ Sechs Jahre nach Ende der Apartheit in Südafrika wurden die Sullivan-Principles 1999 als die „Global Sullivan Principles for Corporate Social Responsibility" neu aufgelegt, um Unternehmen Leitlinien für ihr Handeln vorzuschlagen.

${ }^{46} \mathrm{Köpke} / \mathrm{Röhr}, \mathrm{S}$. 23. Selbstverständlich gab es auch schon weit früher Regelungen von Unternehmen, die unter den nur schwer abgrenzbaren Begriff des Verhaltenskodexes subsumiert werden können. So beispielsweise die Betriebs-, Fabrik- oder Arbeitsordnungen, die Unternehmen Ende des 19. Jahrhunderts erliesen. Inhaltlich handelt es sich dabei aber gerade nicht um unternehmerische Selbstverpflichtungen i. S. v. Verpflichtungen der Unternehmen, sondern vielmehr um Verhaltensmaßgaben der Arbeiter. Vgl. hierzu Willemsen, S. 7 ff. 
Aufgabe der körperlichen Trennung nach Rassen sowie die Verpflichtung zur Fortbildung von „blacks and other nonwhites" und deren Beförderung auf Führungspositionen. ${ }^{47}$ Neben General Motors, dem zu dieser Zeit größten Arbeitsgeber der „Blacks“ in Südafrika, verpflichteten sich mehr als 200 in Südafrika tätige USamerikanische Unternehmen auf diesen Verhaltenskodex. ${ }^{48}$ Ebenfalls im Jahr 1977 beschlossen die neun Mitgliedstaaten der damaligen Europäischen Gemeinschaft einen Verhaltenskodex für Unternehmen mit Tochtergesellschaften, Zweigniederlassungen oder Vertretungen in Südafrika. Dieser wurde von trotz der Freiwilligkeit seiner Umsetzung und entsprechend fehlender Sanktionsmöglichkeiten für Verstöße von dem Großteil der in Südafrika tätigen deutschen Unternehmen eingehalten. ${ }^{49}$

Zwar gab es im Anschluss daran vereinzelte Verhaltenskodizes wie die als Reaktion auf die Katastrophe von Bhopal ${ }^{50}$ vom kanadischen Chemieverband unter der Bezeichnung Responsible-Care veröffentlichten Leitlinien und Prinzipien für verantwortliches Handeln ${ }^{51}$, jedoch kam es erst ab Anfang der 1990er Jahre ausgehend von den USA zu einer signifikanten Verbreitung von Verhaltenskodizes. Hintergrund dieser Verbreitung waren Kampagnen von NROen in diesem Zeitraum, durch welche Missstände bei der Produktion im Hinblick auf Umwelt- und Arbeitsbedingungen aufgedeckt wurden. Insbesondere Markenunternehmen aus der Bekleidungs- und Sportartikelindustrie waren dabei für die zumeist an der Aufdeckung der Missstände mitwirkenden NROen ein attraktives Ziel. Denn sie versprachen einen hohen medialen Effekt und damit Aufmerksamkeit und waren beziehungsweise sind, da sie ihre Produkte zu einem erheblichen Maße aufgrund ihres Images verkaufen, in besonderem Maße auf dieses bedacht. ${ }^{22}$ Aber auch in Europa starteten NROen entsprechende Kampagnen. Dort war zunächst das Bekleidungs- und Handelsunternehmen C\&A betroffen, welchem die niederländische Organisation SOMO53 in ihrem 1989 veröffentlichte Buch „C\&A - De stille Gigant" Arbeitsrechtsverletzungen bei Zulieferern nachwies. ${ }^{54} \mathrm{Um}$ diesen Vorwürfen zu begegnen veröffentlichte C\&A 1995 als eines der ersten europäi-

\footnotetext{
47 Igbanugo, S. 12.

48 Köpke/Rohr, S. 23.

49 Vgl. BT-Drs. 11/1749, Antwort auf Frage 2. Vergleichbare Regelungen gab es auch in Australien und Kanada.

${ }^{50}$ In Bhopal kam es im Jahr 1984 zur Explosion einer Chemiefabrik, durch welche tausende Menschen getötet und eine vielfache Anzahl verletzt wurden.

${ }^{51}$ Eine ausführliche Darstellung des Responsible-Care Programms erfolgt im Rahmen der Beispiele für Verhaltenskodizes in Kapitel 1C.II.2.

52 Biedermann, S. 16, 2007, S. 16 ff. Als erste Unternehmen waren die Firmen Levi Strauss und Nike von entsprechenden Kampagnen betroffen.

${ }^{53}$ SOMO steht für Stichting Onderzoek Multinationale Ondernemingen. Übersetzt bedeutet dies Zentrum für Forschung über multinationale Vereinigungen.

${ }^{54}$ Werner/Weiss, S. 278.
} 
schen Unternehmen einen Verhaltenskodex, der sowohl soziale als auch ökologische Standards in der Lieferkette festlegte. ${ }^{55}$

Ein gutes Beispiel für die Hintergründe der Abgabe eines Verhaltenskodexes sozusagen als Versprechen der Besserung gibt die Entstehungsgeschichte der 1998 verkündeten „Standards of Engagement" von adidas. Nachdem im Vorfeld der Fußball-Europameisterschaft 1996 bekannt wurde, dass die offiziellen Spielbälle teils in Kinderarbeit hergestellt wurden, stand adidas unter starkem Rechtfertigungsdruck. So skandierten beispielsweise 150 Vertreter der US Gewerkschaft United im Rahmen eines Spiels von Steffi Graf bei den US Open 1995 unter den Augen der Weltöffentlichkeit Slogans gegen die Ausbeutung von Kindern und Arbeitern durch adidas. ${ }^{56}$ Dies kam für adidas zu einem besonders ungünstigen Zeitpunkt, da in diesem Zeitraum der Börsengang des Unternehmens vorgesehen war. ${ }^{57}$

Waren entsprechende Verhaltenskodizes trotz der Kampagnen und des entstehenden öffentlichen Drucks auch Anfang der 1990er Jahre noch die Ausnahme, nahm ihre Ausbreitung in den folgenden Jahren massiv zu. So existierten nach einer Untersuchung der ILO im Jahr 1998 weltweit bereits 215 Verhaltenskodizes. ${ }^{58}$ Die OECD zählte im Rahmen einer Studie im Jahr 1999 bereits 233 Verhaltenskodizes. ${ }^{59}$ Aufgrund der starken Ausbreitung verlagerte man sich nachfolgend auf Schätzungen, da eine seriöse Angabe der konkreten Anzahl aufgrund der fehlenden (zentralen) Registrierung unmöglich wurde. So schätzte beispielsweise die Weltbank die Zahl der Kodizes im Jahre 2003 bereits auf ca. 1000.60 Mittlerweile haben Verhaltenskodizes eine derartige Marktdurchdringung erreicht, dass ihr Vorhandensein bei international tätigen Unternehmen nicht mehr die Ausnahme, sondern vielmehr die Regel ist. ${ }^{61}$ Grund hierfür ist, dass sich ein Unternehmen, das auf einen solchen Verhaltenskodex verzichtet, wohl mit öffentlichen Angriffen zu rechnen hätte, die sich geschäftsschädigend auswirken würden. ${ }^{62}$ Inhaltlich betreffen diese Verhaltenskodizes heute zumeist Sozialstandards als Oberbegriff

\footnotetext{
55 Werner/Weiss, S. 278.

56 Artikel des Nachrichtenmagazins FOCUS vom 15.10 .1995 mit dem Titel: „ADIDAS - Stinkende Socken“, http://www.focus.de/finanzen/news/adidas-stinkende-socken_aid_155395.html, zuletzt abgerufen am 24.11.2011.

57 Vgl. Fn. 56.

${ }^{58} \mathrm{ILO}(\mathrm{Hg})$, Overview of global Developments an Office Activities concerning Code of Conduct, Social Labelling and other private sector Initiatives adressing Labour Issues, 1998, GB.273/WP/SDL/1 (Rev.1), 273 ${ }^{\text {rd }}$ Session.

59 OECD (Hg), Codes of Corporate Conduct: An inventory, 1999, S. 4.

${ }^{60}$ Worldbank, Company Codes of Conduct and international Standards: an analytical Comparison, 2003, S. 2.

${ }^{61} \mathrm{Vgl}$. Fn. 9.

${ }^{62}$ Köpke/Rohr, S. 24.
} 
Grundsätzliches zu Verhaltenskodizes

für Standards bei der Ausgestaltung von Arbeitsverträgen im Bezug auf Arbeitszeit, Lohn, soziale Absicherung sowie Arbeitnehmerrechte und Arbeitsschutz. ${ }^{63}$

\section{Kategorien von Verhaltenskodizes}

Verhaltenskodizes treten in den unterschiedlichsten Formen mit den unterschiedlichsten Inhalten auf. Eine Kategorisierung im Hinblick auf die mit ihnen eingegangenen Verpflichtungen birgt die Problematik, dass Verhaltenskodizes inhaltlich durchaus unterschiedliche Bereiche wie beispielsweise den Umweltschutz sowie soziale Mindeststandards umfassen können. Aus diesem Grund empfiehlt es sich, Verhaltenskodizes im Hinblick auf ihre Beteiligten zu kategorisieren. Dabei kann neben der Anzahl der Beteiligten auch auf ihre Funktion abgestellt werden. An diesen Merkmalen orientiert sich die nachfolgende Kategorisierung, innerhalb welcher zur besseren Veranschaulichung innerhalb jeder Kategorie auf ein Beispiel abgestellt wird.

\section{Unternehmenseigene Verhaltenskodizes}

\section{Allgemein}

Sozusagen die Basisversion, aber auch die anteilig weitverbreitetste Variante von Verhaltenskodizes im Hinblick auf die Anzahl und Art der Beteiligten stellen unternehmenseigene Verhaltenskodizes dar. ${ }^{64}$ Entsprechende Verhaltenskodizes betreffen in der Regel nur das Unternehmen selbst ${ }^{65}$, so dass dieses allein über den Inhalt und damit die Qualität des Verhaltenskodexes bestimmt. Hierfür ist auch ohne Belang, dass die jeweiligen Unternehmen bei der Erstellung ihrer Verhaltenskodizes oftmals auf die Expertise von NROen sowie von staatlichen Einrichtungen wie dem „Runden Tisch Verhaltenskodizes“"66 oder der vergleichbaren Ethical Trading Initiative in Großbritannien ${ }^{67}$ zurückgreifen.

${ }^{63}$ Schlussbericht der Enquete-Kommission „Globalisierung der Weltwirtschaft — Herausforderungen und Antworten“", BT.-Drs. 14/9200, S. 169.

${ }^{64}$ Zimmer, S. 149.

${ }^{65}$ Jedoch zumeist unter Einschluss sämtlicher Schwester- und Tochtergesellschaften.

66 Dieser wurde im Januar 2001 ins Leben gerufen wurde. Er versteht sich selbst als ein für Deutschland einmaliges Multistakeholder-Forum im Bereich Verhaltenskodizes und Sozialstandards, mit welchem die teilnehmenden Unternehmen, Wirtschaftsverbände, Gewerkschaften, NROen und nicht zuletzt Bundesministerien unter anderem das Ziel verfolgen, die Sozialstandards in Entwicklungsländern zu verbessern, vgl. die Internetseite des Runden Tisches Verhaltenskodizes, http://www.coc-runder-tisch.de/coc-runder-tisch/, zuletzt aufgerufen am 28.11.2011.

${ }^{67}$ Weitere Informationen unter http://www.ethicaltrade.org/about-eti, zuletzt aufgerufen am 28.11.2011. 
Sollten durch den Verhaltenskodex auch Lieferanten, Subunternehmern und sonstigen Geschäftspartnern verpflichtet werden, sind diese dahingegen zumeist nicht in der Position, inhaltlichen Einfluss auf den Verhaltenskodex zu nehmen. Vielmehr handelt es sich bei entsprechenden Verhaltenskodizes schlussendlich um nichts anderes als Vertragsbedingungen, da ohne ihre Anerkennung eine Auftragsvergabe nicht erfolgt. 68 Üblich ist eine solche Erstreckung auf Lieferanten und Subunternehmer in Branchen wie der Konsumgüter- und Bekleidungsindustrie, in denen die Markenunternehmen oftmals keine eigene Fertigung unterhalten, sondern die Produktion der von ihnen entwickelten und designten Güter bei anderen Unternehmen in Auftrag geben, die oftmals in Entwicklungsländern ansässig sind.

\section{Beispiel: Verhaltenskodizes der Otto Gruppe}

Auf eine solche unternehmenseigene Regelung beruft sich beispielsweise die Otto Gruppe, wobei diese jeweils einen Verhaltenskodex für den Bereich Handelsware und einen Verhaltenskodex für den Bereich Dienstleistungen und NichtHandelsware unterhält. ${ }^{69}$ Die Otto Gruppe ist mit ca. 50.000 Beschäftigten und ca. 11,5 Milliarden Euro Umsatz eines der weltweit größten Handelsunternehmen. ${ }^{70}$ Sie unterhält als Handelsunternehmen keine eigenen Produktionsstätten, sondern kauft die von ihr gehandelten Waren weltweit ein.

\section{a. Verhaltenskodex Handelsware}

Für die Lieferanten ihrer Handelsware initiierte die Otto Gruppe 1996 einen Verhaltenskodex ${ }^{71}$, der 1997 in Kraft trat und seither mehrmals überarbeitet wurde. ${ }^{72}$ Dieser bildet die Grundlage sämtlicher Geschäftsbeziehungen der Otto Gruppe in Bezug auf alle Produktionsabläufe und -stätten für die von ihr bezogenen Handelswaren und gilt für die gesamte Otto Gruppe, deren direkte Geschäftspartner und deren Subunternehmer. ${ }^{73}$ Der Verhaltenskodex beruht dabei inhaltlich im Wesentlichen auf den Anforderungen der Grundsatzerklärung der ILO, der Allgemeinen Erklärung der Menschenrechte der Vereinten Nationen, den UNKonventionen über die Rechte des Kindes und zur Beseitigung jeder Form der

\footnotetext{
${ }^{68}$ Um eine solche Verpflichtung Dritter durchsetzen zu können, bedarf es eines gewissen Einflusses auf diese. Ein solcher besteht in der Regel oftmals dort, wo einzelne Auftraggeber nahezu die gesamte Produktion eines Lieferanten abnimmt bzw. der Hauptauftraggeber des Subunternehmers ist.

${ }^{69}$ Beide Verhaltenskodizes sind abrufbar unter http://www.ottogroup.com/de/die-otto-group/ strategie/corporate-responsibility/lieferanten/code-of-conduct.php.

70 Otto Group, Corporate Responsibility Report 2011, S. 4.

${ }^{71}$ Verhaltenskodex Handelsware, abrufbar vgl. Fn. 69.

${ }^{72}$ Vgl. Fn. 70, S. 96.

${ }^{73}$ Nr. 1 „Geltungsbereich“ des Verhaltenskodex Handelsware der Otto Gruppe, vgl. Fn 69.
} 
Diskriminierung von Frauen, den Prinzipien des UN Global Compact und der OECD-Guidelines. ${ }^{74} \mathrm{Er}$ sieht unter anderem ein Verbot der Kinder- und Zwangsarbeit, der Diskriminierung sowie der Bestechung und Korruption vor. ${ }^{75}$ Darüber hinaus enthält der Verhaltenskodex Handelsware Bestimmungen über die Arbeit von Jugendlichen, die Vereinigungsfreiheit, die zulässigen Arbeitszeiten, die Vergütung, die Gesundheit, die Sicherheit und den Umweltschutz. ${ }^{76}$ Zudem regelt der Verhaltenskodex Handelsware die Verpflichtung zur Einhaltung der jeweiligen nationalen Gesetze beziehungsweise der internationalen Gesetze und Vorschriften $^{77}$, falls letztere strengere Anforderungen stellen. Die Einhaltung dieser durch den Verhaltenskodex Handelsware aufgestellten Grundsätze wird durch die aufgrund des Verhaltenskodexes Verpflichteten garantiert. ${ }^{78}$ Dabei sind die Geschäftspartner der Otto Gruppe für die Einführung eines Managementsystems zur Umsetzung, Einhaltung und Überprüfung zuständig. ${ }^{79}$ Die Überwachung der Einhaltung des Verhaltenskodexes obliegt jedoch der Otto Gruppe. ${ }^{80}$ Stellt sie Verstöße fest, ist der Geschäftspartner zur unverzüglichen Einleitung von Abhilfemaßnahmen verpflichtet. ${ }^{81}$ Für diese hat die Otto Gruppe ihm jedoch ausreichend Zeit zur Verfügung zu stellen und den Geschäftspartner bei diesen zu unterstützen. ${ }^{82}$ Zudem besteht in diesen Fällen ein Recht der Otto Gruppe zur Beendigung der Geschäftsbeziehung. ${ }^{83}$ Jedoch ist eine solche nur ultima ratio. ${ }^{84}$ Vielmehr setzt die Otto Gruppe auch bei Verstößen gegen den Verhaltenskodex auf die Entwicklung und Qualifizierung ihrer Geschäftspartner und auf langfristige Beziehungen. ${ }^{85}$ Hierdurch verspricht sich die Otto Gruppe eine dauerhafte Verbesserung der Verhältnisse in den jeweiligen Ländern. ${ }^{86}$

\footnotetext{
74 Vgl. Fn 75, Präambel.

75 Nr. 1 - 14 des Verhaltenskodex Handelsware der Otto Gruppe, vgl. Fn 69.

76 Vgl. Fn. 75.

77 Hierunter fallen nach Nr. 2 „Einhaltung von Gesetzen“ des Verhaltenskodex Handelsware der Otto Gruppe, vgl. Fn 69 auch ,industrielle Mindeststandards, Konventionen der ILO und der UN sowie andere relevante Bestimmungen."

${ }^{78} \mathrm{Nr}$. 1, letzter Satz „Geltungsbereich“ des Verhaltenskodex Handelsware der Otto Gruppe, vgl. Fn 69.

${ }^{79}$ Nr. 15 „Managementsysteme“ des Verhaltenskodex Handelsware der Otto Gruppe, vgl. Fn 69.

${ }^{80} \mathrm{Nr} .16$ „Überwachung Verhaltenskodex“ des Verhaltenskodex Handelsware der Otto Gruppe, vgl. Fn 69.

${ }^{81} \mathrm{Nr} .17$ „Sanktionen und Abhilfemaßnahmen“ des Verhaltenskodex Handelsware der Otto Gruppe, vgl. Fn 69.

82 Vgl. Fn. 81.

83 Vgl. Fn. 81.

84 Otto Group, Corporate Responsibility Report 2011, S. 99.

85 Vgl. Fn. 84.

86 Vgl. Fn. 84.
} 
b. Verhaltenskodex Dienstleistungen und Nicht-Handelsware

Im März 200987 hat die Otto Gruppe zudem einen weiteren Verhaltenskodex für den Bereich Dienstleistungen und Nicht-Handelsware ${ }^{88}$ erlassen, der jedoch nur die Erwartungen der Otto Gruppe an ihre Geschäftspartner enthält und die Otto Gruppe selbst außen vor lässt. Wie der Verhaltenskodex Handelsware enthält der Verhaltenskodex Dienstleistungen und Nicht-Handelsware zunächst die Verpflichtung zur Einhaltung sämtlicher gesetzlicher Bestimmungen ${ }^{89}$, ein Verbot der Kinderarbeit, Diskriminierung, Bestechung und Korruption sowie Regelungen zur Arbeit von Jugendlichen, zu Arbeiterrechten, zur Vergütung, zur Arbeitssicherheit sowie zum Umweltschutz. ${ }^{90}$ Komprimiert im Vergleich zum Verhaltenskodex Handelsware sind dahingegen die Regelungen zur Überwachung und zum Umgang mit Verstößen. So haben die Geschäftspartner ein Audit bezüglich der Einhaltung nicht zwingend, sondern nur auf Anforderung der Otto Gruppe durchzuführen. Werden Verstöße festgestellt, ist wie beim Verhaltenskodex Handelsware der Geschäftspartner zur Einleitung von Abhilfemaßnahmen verpflichtet. Auch ihm ist dabei jedoch ausreichend Zeit zu gewähren. ${ }^{11}$ Zwar wird das Kündigungsrecht der Otto Gruppe auch bei diesem Verhaltenskodex hierdurch nicht eingeschränkt. Jedoch wird die Kündigungsmöglichkeit in der Praxis ebenfalls nur als letzte Möglichkeit genutzt. ${ }^{92}$

II. Gemeinsame Verhaltenskodizes unabhängiger Unternehmen

1. Allgemein

Eine weitere Variante sind gemeinsame Verhaltenskodizes von unabhängigen Unternehmen. Dabei stammen die beteiligten Unternehmen zumeist aus einem Wirtschaftsbereich. Beteiligt an ihnen sind mehrere oder im Idealfall sogar alle Unternehmen einer Branche aus einer Region, einem Land oder sogar der ganzen Welt. Wie unternehmenseigene Verhaltenskodizes betreffen sie neben dem Verhalten der abgebenden Unternehmen oftmals auch das von Lieferanten, Subunternehmern und sonstige Geschäftspartnern. Vielfach werden solche Verhaltenskodizes auf Anregung oder in Zusammenarbeit mit einem Verband aus der jeweiligen Branche entwickelt. Eine solche gleichlautende Verpflichtung von Unter-

\footnotetext{
87 Otto Group, Corporate Responsibility Report 2011, S. 96.

88 Abrufbar unter http://www.ottogroup.com/de/die-otto-group/strategie/corporate-responsibility /lieferanten/code-of-conduct.php.

${ }^{89}$ Nr. 1 „Einhaltung von Gesetzen“ des Verhaltenskodex Dienstleistungen und Nicht-Handelsware der Otto Gruppe.

${ }^{90}$ Nr. $2-8$ des Verhaltenskodex Dienstleistungen und Nicht-Handelsware der Otto Gruppe.

${ }^{91}$ Nr. 9 „Überwachung Verhaltenskodex“ des Verhaltenskodex Dienstleistungen und NichtHandelsware der Otto Gruppe.

92 Vgl. Fn. 84.
} 
Grundsätzliches zu Verhaltenskodizes

nehmen einer Branche hat den Vorteil, dass die mit einem Verhaltenskodex einhergehenden wirtschaftlichen Nachteile wie beispielsweise höhere Produktionskosten aufgrund von erhöhten Umweltstandards zumindest die beteiligten Unternehmen in gleichem Maße belasten, so dass kein Wettbewerbsnachteil entsteht. ${ }^{93}$

\section{Beispiel: Responsible Care Programme des VCI und des VCH}

Eine solche Branchenregelung ist das Responsible Care (zu Deutsch: verantwortliches Handeln) Programm der Chemischen Industrie. Es wurde im Jahr 1985 vor dem Hintergrund der Katastrophe von Bhupol/Indien ${ }^{94}$ vom kanadischen Chemieverband ${ }^{95}$ begründet. Mittlerweile hat es sich zu einer internationalen Initiative entwickelt, der sich aktuell Verbände der Chemischen Industrie aus 53 Ländern angeschlossen haben. ${ }^{96}$ Ziel des Responsible Care Programms ist die stetige Verbesserung in den Bereichen Gesundheit, Sicherheit und Umweltschutz über die gesetzlichen Anforderungen hinaus sowie eine offene Kommunikation mit allen Stakeholdern. ${ }^{97}$ Dabei ist eines der neun Schlüsselelemente des unter dem Dach des Chemieweltverbandes ${ }^{98}$ angesiedelten Programms, dass die internationalen Anforderungen durch die nationalen Chemieverbände in nationale Responsible Care Programme umgesetzt werden. ${ }^{99}$ Bei dieser Umsetzung haben die nationalen Verbände jedoch aufgrund der allgemeingehaltenen Leitlinien einen relativen weiten Spielraum. Dies zeigt sich deutlich an den zwei in Deutschland bestehenden Responsible Care Programmen des VCI und des VCH, auch wenn die Verbände diesbezüglich unter Einbindung des Verbandes der europäischen Chemischen Industrie kooperieren. So ist jedes Mitglied des VCI ${ }^{100}$ aufgrund eines Mitgliederbeschlusses automatisch Mitglied im 1995 eingeführten ${ }^{101}$ Responsible Care Programms des Verbandes, ohne das es eines formalen Beitrittsaktes bedarf. ${ }^{102}$ Damit ist jedes Mitgliedsunternehmen grundsätzlich verpflichtet, die Responsible Care

\footnotetext{
93 Selbstverständlich können aber Wettbewerbsnachteile gegenüber solchen Unternehmen bestehen, die sich nicht auf den Verhaltenskodex der Branche verpflichten.

${ }^{94}$ In Bhupol/Indien kam es im Jahr 1984 zu einem Unfall in der Chemiefabrik eines amerikanischen Unternehmens, durch welchen mehrere tausend Menschen zu Tode kamen und eine nicht zu beziffernde Anzahl an Menschen schwere gesundheitliche Schäden erlitt, vgl. Kapitel 1B.

${ }^{95}$ Belanger/Topalovic/Krantzberg, S. 6.

${ }^{96}$ Information des VCI, vgl. https://www.vci.de/Nachhaltigkeit/Responsible-Care/Initiative/ Seiten/Responsible-Care-im-Ueberblick.aspx, zuletzt aufgerufen am 30.01.2013

${ }^{97}$ Einleitung Responsible Care Global Charter, abrufbar unter http://www.icca-chem.org/Home/ ICCA-publications $/$ Publications-Search-Results $/$ type $=$ Strategy+Document, zuletzt abgerufen am 30.01.2013.

98 International Council of Chemical Associations, http://www.icca-chem.org/.

${ }^{99}$ Schlüsselelement 2 der Responsible Care Global Charter.

100 Einschließlich der Fachverbände.

${ }_{101}$ Daniel, Chemie Report Special, 09/2011, S. 2 f.

102 Der VCI wirkt jedoch gegenüber seinen Mitgliedern auf die Abgabe förmlicher Teilnahmeerklärungen sowie auf eine öffentliche Bekanntgabe der Teilnahme hin.
} 
Leitlinien des VCI 103 einzuhalten, welche im Jahr 2007 auf Grundlage der 2006 in Dubai verabschiedeten Responsible Care Global Charter ${ }^{104}$ überarbeitet ${ }^{105}$ wurden. Diese betreffen insbesondere den Schutz von Mensch und Umwelt sowie die Information der Öffentlichkeit. Wesentlich ist dabei die Ankündigung, die Beteiligten Unternehmen würden „ungeachtet der wirtschaftlichen Interessen die Vermarktung von Produkten einschränken oder deren Produktion einstellen, falls nach den Ergebnissen einer wissenschaftlichen Risikobewertung die Vorsorge zum Schutz vor Gefahren für Gesundheit und Umwelt dies erfordert."106 Eine Kontrolle der Einhaltung der Leitlinien seines Responsible Care Programms nimmt der VCI jedoch nicht vor und greift allenfalls ein, wenn das Responsible Care Logo unberechtigt genutzt wird. Ebenso gibt es keine verbindliche Berichtspflicht über die Umsetzung der Leitlinien. ${ }^{107}$ Entsprechend ist die grundsätzlich bestehende Möglichkeit eines Verbandsausschlusses aufgrund eines Verstoßes gegen die Leitlinien nur in Extremfällen zu erwarten.

Dahingegen baut das Responsible Care Programm des $\mathrm{VCH}^{108}$ auf einer förmlichen Beitrittserklärung auf ${ }^{109}$ und stellt konkrete Forderungen an die Teilnehmer, damit diese das Responsible Care Logo führen dürfen. So müssen die teilnehmenden Unternehmen zunächst einen Responsible Care Beauftragten benennen. ${ }^{110}$ Dieser muss sodann eine Selbsteinschätzung des Unternehmens anhand eines standardisierten Fragebogens abzugeben, die von einem sachverständigen Dritten auf Plausibilität geprüft wird und bei Beanstandung korrigiert werden kann.111 Erscheint die Beantwortung auch nach einer zweiten Beanstandung nicht plausibel, verliert das Unternehmen die Berechtigung zur Verwendung des Logos. ${ }^{112}$ In

103 Abrufbar unter https://www.vci.de/Themen/Umwelt-Sicherheit/Responsible-Care/Seiten/ Leitlinien-des-deutschen-Responsible-Care-Programms-2007.aspx\#, zuletzt abgerufen am 30.01.2013.

104 Abrufbar unter http://www.icca-chem.org/Home/ICCA-publications/Publications-SearchResults/?type $=$ Strategy + Document, zuletzt abgerufen am 30.01.2013.

105 Vgl. Fn. 96.

106 Punkt 7 der Leitlinien des deutschen Responsible Care Programms.

107 Trotzdem beteiligen sich an den jährlichen Berichten jeweils ca. 800 Mitgliedsunternehmen, die wiederum ca. 90\% der Beschäftigen in der chemischen Industrie in Deutschland repräsentieren, vgl. Fn. 96.

108 Wie im Übrigen auch das Responsible Care Programm des Fachverbandes der Chemischen Industrie Österreichs, somit des Pendants des VCI, vgl. hierzu http://www.fcio.at/DE/fcio/Schwerpunktthemen/Responsible $\% 20$ Care $\% 20 \% 28$ RC $\% 29$ /Responsible+Care.aspx, zuletzt abgerufen am 31.01.2013.

109 Punkt 1 „Teilnahmeerklärung der Geschäftsleitung“ des Responsible Care Programms des VCH, abrufbar unter http://www.vch-online.de/index.php?option=com_content\&task=view \&id=20\&Itemid=27, zuletzt abgerufen am 30.01.2013.

110 Punkt 2 „Bestellung eines Responsible Care Beauftragten“ des Responsible Care Programms des $\mathrm{VCH}$.

111 Punkt 4 „Selbsteinschätzung anhand eines Fragebogens“ des Responsible Care Programms des $\mathrm{VCH}$.

112 Vgl. Fn. 111. 
einem auf der Selbsteinschätzung aufbauenden weiteren Schritt muss jedes Unternehmen einen Dreijahresplan aufstellen, der kontinuierliche Verbesserungen in den Bereichen Sicherheits-, Gesundheits- und Umweltschutz umfasst und jährlich vom Responsible Care Beauftragten des Unternehmens geprüft werden muss. ${ }^{113}$ Auch dieser Dreijahresplan wird wie seine Erfüllung nach Ablauf der Dreijahresfrist von einem unabhängigen, sachverständigen Dritten ${ }^{114}$ geprüft. ${ }^{115}$ Ergibt diese Überprüfung nach drei Jahren, dass eine kontinuierliche Verbesserung nicht eingetreten ist, bleibt dem Unternehmen eine Nachfrist von sechs Monaten zur Vornahme der notwendigen Korrekturen. ${ }^{116}$ Reichen diese nach Einschätzung des Sachverständigen abermals nicht aus, hat das Unternehmen unter Hilfestellung des VCH eine weitere Chance zur Korrektur, wobei der Nachbesserungszeitraum auf insgesamt zwölf Monate begrenzt ist. ${ }^{117}$ Kann auch dann keine entsprechende Verbesserung festgestellt werden, wird ebenfalls die Berechtigung zur Nutzung des Logos entzogen. ${ }^{118}$ Darüber hinaus sind die teilnehmenden Unternehmen verpflichtet, jährlich über Umsetzung der Leitlinien in die praktische Arbeit des Unternehmens zu berichten ${ }^{119}$ und alle Maßnahmen im Rahmen des Programms zu dokumentieren. ${ }^{120}$ Als Bestandteil des Responsible Care Programms des VCH sind darüber hinaus die „Leitlinien des Chemiehandels zum sicheren Umgang mit Chemikalien, deren Vermarktung sowie zur Unterrichtung der Öffentlichkeitt"121 umzusetzen. Diese von der Mitgliederversammlung des VCH bereits 1995 beschlossenen Leitlinien enthalten unter anderem Verhaltensvorgaben zur Anlagen- und Transportsicherheit ${ }^{122}$, zur Abgabe von (besonders sensiblen) Chemikalien ${ }^{123}$, zur Information der Öffentlichkeit ${ }^{124}$ sowie die strikte Einhaltung der eingegangen Verhaltenskodizes und Selbstverpflichtungsabkommen. ${ }^{125}$

\footnotetext{
113 Punkt 5 „Kontinuierliche Verbesserung - Aufstellung eines Dreijahresplanes und jährliche Überprüfungen" des Responsible Care Programms des VCH.

114 Die Anforderungen an den Sachverständigen werden in Punkt 8 „Verifizierung durch sachverständigen Dritten“ des Responsible Care Programms des VCH festgelegt.

115 Punkt 6 „Widerruf des Logos“ des Responsible Care Programms des VCH.

$116 \mathrm{Vgl}$. Fn. 115.

117 Vgl. Fn. 115.

118 Vgl. Fn. 115

119 Punkt 7 „Indikatoren zur Leistungsmessung“ des Responsible Care Programms des VCH.

120 Punkt 9 „Dokumentation“ des Responsible Care Programms des VCH.

121 Diese Leitlinien sind abrufbar unter http://www.vch-online.de/index.php?option= com_content\&task $=$ view\&id $=28 \&$ Itemid $=60$, zuletzt abgerufen am 30.01.2013.

$122 \mathrm{Nr} .2$ und 3 der Leitlinien.

$123 \mathrm{Nr} .4$ und 5 der Leitlinien.

124 Nr. 6 der Leitlinien.

125 Nr. 8 der Leitlinien.
} 
III. Verhaltenskodizes von Unternehmen unter Beteiligung unabhängiger, nichtwirtschaftlicher Dritter

\section{Allgemein}

Die Kategorie mit dem höchsten Renommee für Unternehmen sind Verhaltenskodizes, die gemeinsam mit einer unabhängigen, nichtwirtschaftlichen Organisation abgeschlossen werden. Hierbei kann es sich um Gewerkschaften, NROen, staatliche Organisationen und sogar Staaten handeln. Entsprechende Verhaltenskodizes sind jedoch im Verhältnis zu den vorgenannten Kategorien deutlich seltener. Grund hierfür ist, dass die unabhängigen, nichtwirtschaftlichen Organisationen oftmals auf verbindliche Regelungen bestehen, die Unternehmen jedoch keine verbindlichen Regelungen eingehen wollen. Die unabhängigen, nichtwirtschaftlichen Organisationen wiederum wollen ihre Reputation, die im Wesentlichen auf ihrer Unabhängigkeit und ihrem Idealismus gründet, nicht dadurch gefährden, dass sie als Steigbügelhalter der Imagepflege von Unternehmen wahrgenommen werden. ${ }^{126}$ Aus diesem Grund sehen diese Organisationen und insbesondere die NROen ihre Aufgabe hauptsächlich in der Kontrolle der Einhaltung der Verhaltenskodizes von Unternehmen und der Veröffentlichung von festgestellten Verstößen. Diese Aufgabe kann in ihrer Bedeutung nur schwerlich überschätzt werden, da allein die Gefahr einer entsprechenden Kampagne über die Nichteinhaltung ihrer Verhaltenskodizes die Unternehmen zu mehr Disziplin anhält. ${ }^{127}$

\section{Beispiel: UN Global Compact}

Der UN Global Compact (Globaler Pakt der Vereinten Nationen) geht auf eine Initiative der Vereinten Nationen zurück, mit welcher sich diese erstmals nicht an Staaten, sondern direkt an private Organisationen gewandt haben. So forderte der damalige Generalsekretär der Vereinten Nationen Kofi Annan im Rahmen einer Rede im Januar 1999 auf dem Weltwirtschaftsforum in Davos die Vertreter der anwesenden Wirtschaftsunternehmen auf, gemeinsam mit den Vereinten Nationen einen Pakt über die gemeinsamen Werte und Prinzipien aus den Bereichen Menschenrechte, Arbeitsstandards und Umweltschutz zu schließen. ${ }^{128}$ Unter dem sanften Druck der Androhung einer verbindlichen internationalen Regelung ${ }^{129}$

\footnotetext{
${ }^{126}$ So steht insbesondere der WWF als eine der größten internationalen Naturschutzorganisationen ob seiner Nähe zur Industrie und seiner Finanzierung durch Großspender aus der Wirtschaft in der Kritik, vgl. hierzu insbesondere Huismann, Schwarzbuch WWF — Dunkle Geschäfte im Zeichen des Pandas.

127 Vgl. Kapitel 1B.

128 Wortlaut der Rede abrufbar unter http://www.un.org/News/Press/docs/1999/ 19990201.sgsm6881.html, zuletzt abgerufen am 31.01.2013.

${ }^{129}$ Vgl. Fn. 128
} 
erklärten viele führende Unternehmen ${ }^{130}$ aus den verschiedensten Branchen und Regionen sowie mehrere NROen, Verbände und Gewerkschaften ihre Unterstützung der Initiative. ${ }^{131}$ Dies führte zu einer zeitnahen Gründung des Global Compact am 26.07.2000 unter Geltung von zunächst neun Prinzipen aus den in der Rede von Kofi Annan in Davos angekündigten Bereichen. Dabei stammen die Prinzipien $1^{132}$ und $2^{133}$ über Menschenrechte aus der Allgemeinen Erklärung der Menschenrechte der Vereinten Nationen aus dem Jahr 1948, die Prinzipien 3 bis $6^{134} \mathrm{zu}$ Arbeitsbeziehungen aus der Erklärung über grundlegende Prinzipien und Rechte bei der Arbeit der ILO und die Prinzipien 7 bis 9135 zum Umweltschutz aus der Erklärung von Rio de Janeiro zu Umwelt und Entwicklung. Die Prinzipien wurden dabei bewusst aus bestehenden und allgemein anerkannten Regelwerken entnommen, um deutlich zu machen, dass der UN Global Compact keine neuen Regelungen aufstellt, sondern lediglich Bezug auf als allgemeingültig anerkannte Werte nimmt. ${ }^{136}$ Diesen neun Prinzipien wurde 2004 als zehntes Prinzip die Korruptionsbekämpfung hinzugefügt. ${ }^{137}$

Mit ihrer Teilnahme am Global Compact verpflichten sich die an ihm beteiligten Wirtschaftsunternehmen ${ }^{138}$,

${ }^{130}$ Gründungsmitglieder waren unter anderem die deutschen Großkonzerne BASF, Bayer, Daimler und die Deutsche Bank.

131 Fenner, S. 53.

132 Prinzip 1: Unternehmen sollen den Schutz der internationalen Menschenrechte unterstützen und achten.

133 Prinzip 2: Unternehmen sollen sicherstellen, dass sie sich nicht an Menschenrechtsverletzungen mitschuldig machen.

134 Prinzip 3: Unternehmen sollen die Vereinigungsfreiheit und die wirksame Anerkennung des Rechts auf Kollektivverhandlungen wahren.

Prinzip 4: Unternehmen sollen sich für die Beseitigung aller Formen der Zwangsarbeit einsetzen.

Prinzip 5: Unternehmen sollen sich für die Abschaffung von Kinderarbeit einsetzen.

Prinzip 6: Unternehmen sollen sich für die Beseitigung von Diskriminierung bei Anstellung und Erwerbstätigkeit einsetzen.

135 Prinzip 7: Unternehmen sollen im Umgang mit Umweltproblemen dem Vorsorgeprinzip folgen. Prinzip 8: Unternehmen sollen Initiativen ergreifen, um größeres Umweltbewusstsein zu fördern.

Prinzip 9: Unternehmen sollen die Entwicklung und Verbreitung umweltfreundlicher Technologien beschleunigen.

136 Vgl. Fn. 131, S. 55.

137 Prinzip 10: Unternehmen sollen gegen alle Arten der Korruption eintreten, einschließlich Erpressung und Bestechung.

138 Pressemitteilung der UNO (UNIC/275): „Der Globale Pakt - Eine Initiative von UNOGeneralsekretär Kofi Annan zur Einbindung der Privatwirtschaft und der Zivilgesellschaft in die Bemühungen zur Bewältigung der Globalisierung.", abrufbar unter http://www.unric.org/de/uno-und-privatwirtschaft-pressemitteilungen/4543, zuletzt abgerufen am 31.01.2013. 
1) eine eindeutige Unterstützungserklärung für den „Globalen Pakt“ und seine Grundsätze abzugeben und sich öffentlich für den Pakt einzusetzen;

2) einmal im Jahr auf der Internetseite des „Globalen Paktes“ (www.unglobalcompact.org) ein konkretes Beispiel bei der Umsetzung der Prinzipien erzielter Fortschritte oder gemachter Erfahrungen - sei es durch Änderungen in der internen Unternehmensführung oder durch konkrete betriebliche Erfahrungen - zu veröffentlichen.

3) sich in Partnerschaft mit UNO-Organisationen bei Vorhaben zu engagieren, die der Umsetzung der Prinzipien dienen, oder in strategische Partnerschaften zur Förderung großer UNO-Ziele, wie etwa der Beseitigung der Armut, einzutreten.

Mittlerweile hat der UN Global Compact mehr als 10.000 Beteiligte, wovon mehr als 7.000 Unternehmen oder Unternehmensorganisationen sind. ${ }^{139}$ Allerdings sieht der UN Global Compact keine Überwachung der Einhaltung dieser Verpflichtungen und auch keine Sanktionen für Verstöße gegen diese vor. ${ }^{140}$ Dies betrifft nicht nur die Einhaltung der zehn Prinzipien, sondern bereits die Abgabe des jährlichen Berichts. ${ }^{141}$ Gerade diese fehlende Verbindlichkeit ist neben der weiten Formulierung der Prinzipien Hauptgegenstand der Kritik am UN Global Compact, die hauptsächlich von NROen vorgebracht wird. ${ }^{142}$

\section{Bindungswirkung von Verhaltenskodizes}

Die Bindungswirkung von Verhaltenskodizes kann nicht pauschal festgestellt werden, sondern nur für jeden Einzelfall durch die Analyse seiner konkreten Ausgestaltung, die jeweils allein den an dem Verhaltenskodex Beteiligten obliegt. Denn Verhaltenskodizes unterliegen als Regelungen des Privatrechts dem Prinzip der Privatautonomie und der in dieser enthaltenden Vertragsfreiheit, welche wiederum Ausfluss der durch Art. 1, 2 GG garantierten allgemeinen Handlungsfreiheit ist. ${ }^{143}$ Die Parteien sind somit in der Ausgestaltung ihrer Verhaltenskodizes grundsätzlich frei ${ }^{144}$, soweit die Verhaltensmaßgaben nicht gegen Gesetze verstoßen. Diese

\footnotetext{
139 Stand 23.10.2012, vgl. http://www.unglobalcompact.org/ParticipantsAndStakeholders /index.html, zuletzt abgerufen am 31.01.2013.

140 Aus diesem Fehlen an Überwachung und Sanktionen folgert die UNO, dass der UN Global Compact keinen Verhaltenskodex darstelle, vgl. Fn. 138. Diese Schlussfolgerung widerspricht jedoch dem allgemeinen Sprachgebrauch des Begriffs Verhaltenskodex, der keine Überwachung und Sanktionen voraussetzt, vgl. Kapitel 1A.

141 Vgl. Fn. 131, S. 60 in Fn. 132.

142 Vgl. Fn. 131, S. 88.

143 Olzen in: Staudinger, BGB, Einleitung zum Schuldrecht, Rn. 49.

${ }^{144}$ Frenz, S. 102.
} 
Gestaltungsfreiheit ist nur in den in der Praxis höchst seltenen Fällen eingeschränkt, in welchen der Staat als Partner an dem Verhaltenskodex beteiligt ist ${ }^{145}$ oder zumindest über seinen Inhalt bestimmt. ${ }^{146}$ Die Beurteilung der Bindungswirkung muss daher auf Grundlage der allgemeinen Regelungen des Zivilrechts erfolgen. Insofern setzt eine Bindungswirkung an die in einem Verhaltenskodex benannten Verhaltensweisen voraus, dass der Verhaltenskodex tatsächlich eine Verpflichtung auf diese enthält. Auch wenn die Allgemeinheit eine solche Verpflichtung nach ihrem Verständnis des Begriffs wohl voraussetzt ${ }^{147}$, wird eine solche Verpflichtung im rechtlichen Sinne in einer Vielzahl von Verhaltenskodizes umgangen. So beispielsweise indem der Verhaltenskodex lediglich eine Strebsamkeits148 oder Unterstützungserklärung ${ }^{149}$ enthält oder ausdrücklich als unverbindlich gekennzeichnet wird.

Aber selbst wenn der Verhaltenskodex eine eindeutige Verpflichtung enthält ${ }^{150}$, bedeutet dies keine Bindungswirkung des abgebenden Unternehmens in der Gestalt, dass ein unabhängiger Dritter die Einhaltung der Verpflichtung beanspruchen kann. Denn im Zivilrecht stellt sich nicht nur die Frage, wozu man sich verpflichtet hat, sondern auch, wem gegenüber man verpflichtet ist. Letzteres ist insbesondere bei unternehmenseigenen Verhaltenskodizes ${ }^{151}$ problematisch, da diese hierzu in aller Regel keine Angaben enthalten. Entsprechend ergibt sich die tatsächliche Unverbindlichkeit solcher unternehmenseigener Verhaltenskodizes, die den Großteil der vorhandenen Verhaltenskodizes darstellen ${ }^{152}$, selbst bei Vorliegen einer entsprechend verbindlich auszulegenden Formulierung aus dem Fehlen eines Anspruchsberechtigten. ${ }^{153}$ Anders ist die Situation dahingegen bei Verhaltenskodizes mit mehreren Beteiligten, da diese die Grundvoraussetzung eines Vertrages - die Beteiligung von mindestens zwei Parteien ${ }^{154}$ - als Voraussetzung für die Begründung eines Schuldverhältnisses ${ }^{155}$ erfüllen. Dies bedeutet je-

\footnotetext{
145 Vgl. Kapitel 1C.III.

146 Vgl. Fn. 144.

147 Vgl. Kapitel 1A.

148 Im Sinne von: Das Unternehmen XY strebt an, die ILO-Kernarbeitsnormen einzuhalten sowie einen zur Deckung der jeweiligen örtlichen Lebensverhältnisse ausreichenden Lohn zu zahlen.

149 Wie bspw. der UN Global Compact, vgl. Kapitel 1C.III.2, Nr. 1 der von den Unternehmen abzugebenden Verpflichtungen.

150 Im Sinne von: Das Unternehmen XY verpflichtet sich, die ILO-Kernarbeitsnormen einzuhalten sowie einen zur Deckung der jeweiligen örtlichen Lebensverhältnisse ausreichenden Lohn zu zahlen

151 Vgl. Kapitel 1C.I.

152 Zimmer, S. 149.

153 Knauff, S. 249, Beyer, S. 271, a. A. Frenz, S. 99 ff., S. 228 ff., der entsprechende Selbstverpflichtungen aufgrund ihrer öffentlichen Bekanntgabe wie empfangsbedürftige Willenserklärungen

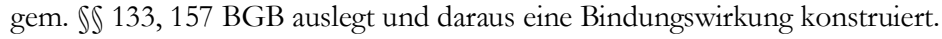

154 Frenz, S. 88.

155 Dies ergibt sich aus $₫ 311$ Abs. 1 BGB.
} 
doch nicht, dass jeder Verhaltenskodex mit mindestens zwei Parteien als Vertrag einzuordnen wäre. Vielmehr ist dies nur dann der Fall, wenn der Verhaltenskodex eine Verpflichtung eines Beteiligten gegenüber einem anderen Beteiligten enthält und somit als Vertrag einzuordnen ist. ${ }^{156}$ Dies ist dann nicht der Fall, wenn ein Verhaltenskodex wie die unternehmenseigenen Verhaltenskodizes als unilaterale, d. h. als einseitige Verpflichtungserklärung einzuordnen ist. Zudem bedeutet auch die Einordnung als Vertrag nicht zwangsläufig, dass das verpflichtete Unternehmen zur Einhaltung der niedergelegten Verhaltensweisen gezwungen werden kann. Denn auch die Sanktionierung für den Verstoß gegen eine Verpflichtung unterliegt der Vertragsfreiheit der Beteiligten. Hierbei kommen insbesondere Strafzahlungen oder ein Ausschluss aus dem Verhaltenskodex in Betracht. ${ }^{157} \mathrm{Zu}$ dem besteht die Bindungswirkung bei Verträgen grundsätzlich nur zwischen den Parteien, so dass eine Durchsetzung der vertraglichen Verpflichtungen durch unbeteiligte Dritte ausscheidet. Aus diesem Grund sind die oftmals in Verhaltenskodizes enthaltenen Klauseln, wonach von Dritten keinerlei Ansprüche aus diesen geltend gemacht werden können ${ }^{158}$, lediglich klarstellender Natur.

Als rein privatrechtliche Regelungen können Verhaltenskodizes auch nicht dem sogenannten Soft Law zugerechnet werden. ${ }^{159}$ Denn Voraussetzung für die Zuordnung einer Regelung zum Soft Law ist die Mitwirkung eines Hoheitsträgers, so dass allein von Privaten geschaffene Regelungen nicht umfasst sind. ${ }^{160}$ Zwar ist der Begriff des Soft Law nicht verbindlich definiert ${ }^{161}$, jedoch ergibt sich die $\mathrm{V}_{\mathrm{O}-}$ raussetzung der Beteiligung eines Hoheitsträgers unter Rückgriff auf die ursprüngliche Begriffsverwendung im Völkerrecht. ${ }^{162}$ Dort wurde der Begriff nur für solche Regelungsformen verwendet, die auf Völkerrechtssubjekte rückführbar waren. ${ }^{163}$ In der Folgezeit wurden dahingegen die unterschiedlichsten Regelungen unter den Begriff des Soft Laws gefasst. Dabei reichte es oftmals in Form einer

156 Frenz, S. 86 ff., 197 ff., Knauff, S. 249.

157 So auch in dem unter Kapitel 1C.II.2 gewählten Beispiel des Responsible Care Programm des $\mathrm{VCH}$.

158 So bspw. in der VW Sozialcharta (abrufbar unter http://www.vwgroupsupply.com/b2bpub/ zusammenarbeit/nachhaltigkeit/leitlinien.html, zuletzt abgerufen am 05.02.2013, welche zwischen dem Vorstand, dem Weltkonzernbetriebsrat und dem internationalen Metallgewerkschaftsbund geschlossen wurde. Dort heißt es in $\ 2$ Abs. 4: „Aus dieser Erklärung können durch Dritte keinerlei Ansprïche geltend gemacht werden."

159 Knauff, S. 241 ff.

${ }^{160}$ Für eine Zuordnung zum Soft Law dahingegen: Walter, in: M. Lang/J. Schuch/ C. Staringer, Soft Law in der Praxis, S. 23 ff., Schlussbericht der Enquete-Kommission „Globalisierung der Weltwirtschaft - Herausforderungen und Antworten“, S. 443 ff., Europäisches Parlament, Arbeitsdokument zu institutionellen und rechtlichen Auswirkungen der Anwendung der Instrumente des „Soft Law“, v. 22.02.2007 (Berichterstatter: Manuel Medina Ortega), PE 384.581v02-00, Dokumentennummer: 653346, S. 2.

161 Arndt, S. 39 ff. unter Darstellung verschiedener Ansätze zur Definition des Begriffs.

162 Vgl. Fn. 159, S. 217.

163 Vgl. Fn. 159, S. 217. 
negativen Abgrenzung als Begründung aus, dass Regelungen — wie die Selbstregulierung durch Private — nicht dem vom traditionellen (demokratisch legitimierten) Gesetzgeber geschaffenen, kodifizierten und damit rechtsverbindlichen Hard Law ${ }^{164}$ zugeordnet werden konnte. ${ }^{165}$ Dies führt jedoch zu einem Konturverlust des Begriffs Soft Law, der nicht durch einen damit einhergehenden Erkenntnisgewinn gerechtfertigt werden kann. ${ }^{166}$ Wenn überhaupt kommt eine Zuordnung zum Soft Law daher nur für die Sonderfälle eines Verhaltenskodexes unter staatlicher Beteiligung in Betracht. ${ }^{167}$ Schlussendlich ist dies jedoch zumindest für die Bindungswirkung unerheblich, da die Unverbindlichkeit begrifflicher Bestandteil des Soft Law ist. ${ }^{168}$ Entsprechend ist die Unverbindlichkeit auch Bestandteil der für die Europäische Union maßgeblichen ${ }^{169}$ Definition des Soft Law von Senden ${ }^{170}$ als „,...) rules of conduct that are laid down in instruments which have not been attributed legally binding force as such, but nevertheless may have certain (indirect) legal effects, and that are aimed at and may produce practical effects. "171

\footnotetext{
164 Arndt, S. 39 ff.

165 Schlussbericht der Enquete-Kommission „Globalisierung der Weltwirtschaft — Herausforderungen und Antworten" unter Verweis auf die Europäische Kommission, S. 444.

166 Vgl. Fn. 159, S. 218.

167 Vgl. Fn. 159, S. 218 f.

168 Vgl. Fn. 159, S. 225.

169 Sie bildet die Grundlage des Arbeitsdokumentes des Europäischen Parlamentes zum Soft Law, vgl. Fn. 160.

170 Senden, S. 112.

171 Übersetzung entsprechend Fn. 170, S. 2: „V Verhaltensregeln, die in Instrumenten enthalten sind, denen keine verbindliche Rechtswirkung als solche verliehen wurde, die aber dennoch bestimmte - indirekte - rechtliche Wirk.ungen haben und durch die Wirkungen in der Praxis bezweckt und unter Umständen ausgelöst werden. "
} 


\section{Kapitel 2}

\section{Die Bedeutung von Verhaltenskodizes im UWG}

\section{A. Unter dem UWG 1909 und dem UWG 2004}

Verhaltenskodizes spielten im deutschen Lauterkeitsrecht bis zur Reformierung des UWG im Jahr 2008 zur Umsetzung der UGP-Richtlinie nur eine sehr eingeschränkte Rolle. So war der Begriff selbst dem deutschen Lauterkeitsrecht bis dahin unbekannt. ${ }^{172}$ Inhaltlich waren Verhaltenskodizes jedoch Gegenstand der Literatur zum Lauterkeitsrecht sowie gerichtlicher Auseinandersetzungen, soweit es sich bei ihnen um „Wettbewerbsrichtlinien“"173 beziehungsweise „Wettbewerbsregeln“'174 handelte. Dabei mussten Verhaltensweisen im Zusammenhang mit Verhaltenskodizes wie beispielsweise der Verstoß gegen einen Verhaltenskodex mangels Spezialregelungen unter die allgemeinen Vorschriften des jeweils gültigen UWG subsumiert werden. Dies erfolgte auf Grundlage des UWG 1909 über den in die-

\footnotetext{
172 Begründung der Bundesregierung für den Gesetzentwurf zur Änderung des UWG vom 20.08.2008, BT.-Drs. 16/10145, S. 12.

${ }^{173}$ Urteil des BGH 08.11.1990 - I ZR 48/89 (OLG Hamm) (Wettbewerbsrichtlinie der Privatwirtschaft) in GRUR 1991, 462.

${ }^{174}$ Urteil des BGH v. 7. 2. 2006 - KZR 33/04 (OLG Hamburg) (Probeabonnement) in NJW 2006, $2627 \mathrm{ff}$.
} 
sem maßgeblichen Begriff der, „guten Sitten“ aus der Generalklausel des $\ 1$ UWG 1909. Da bei deren Ermittlung die im Verkehr bestehenden Auffassungen und dabei insbesondere die des verständigen, durchschnittlichen Gewerbetreibenden zugrunde gelegt wurden, griffen die Gerichte gerne auf Erklärungen der organisierten Marktbeteiligten zu bestimmten Verhaltensweisen sowie auf deren Wettbewerbsrichtlinien zurück. ${ }^{175}$ Zwar verneinte der BGH dabei einen Automatismus (Verstoß gegen Wettbewerbsrichtlinie = Unlauterkeit), gestand Wettbewerbsrichtlinien jedoch eine Indizwirkung bei der Beurteilung der (Un-)Lauterkeit des ursächlichen Verhaltens zu. ${ }^{176}$ Die Beschränkung auf eine Indizwirkung begründete der BGH dabei damit, dass Wettbewerbsrichtlinien ansonsten anstelle des Gesetzes treten würden ${ }^{177}$ und die aktuellen Wettbewerber zudem mit einer besonders strengen Wettbewerbsrichtlinie die Freiheit des Wettbewerbs beschränken könnten, was den Zielen des UWG entgegenlaufen würde. ${ }^{178}$ Trotz dieser Rechtsprechung kam die Indizwirkung einem solchen Automatismus jedoch in manchen Entscheidungen des BGH sehr nahe. ${ }^{179}$ Dies veranlasste Sosnitza eine anfänglich „allizu willfährige Übernabme derartiger Regelwerke durch die Rechtsprechung" zu beklagen, die sich jedoch auch nach seiner Ansicht in eine deutlich kritischere Haltung wandelte. ${ }^{180} \mathrm{Im}$ Jahr 2006 verdeutlichte der BGH seine Rechtsprechung bezüglich der Auswirkungen von Verstößen gegen Verhaltenskodizes unter Zugrundelegung der geänderten Rechtslage des UWG 2004181 mit seiner ProbeabonnementEntscheidung ${ }^{182}$, indem er den oben dargestellten Automatismus auch für die Fall verneinte, in welchen die Wettbewerbsregel, gegen die verstoßen wurde, von der Kartellbehörde als solche des GWB anerkannt worden war. ${ }^{183}$ Zur Begründung führte der BGH aus, dass es verfassungsrechtlich bedenklich sei, „wenn Wettbewerbsregeln zur Ausfuillung der lauterkeitsrechtlichen Generalklauseln und zur Konkretisierung unbestimmter Rechtsbegriffe herangezogen würden. "184 Gleichzeitig schwächte der BGH

\footnotetext{
175 Sosnitza, S. 212.

176 Urteil des BGH 08.11.1990 - I ZR 48/89 (OLG Hamm) (Wettbewerbsrichtlinie der Privatwirtschaft) in GRUR 1991, 462.

177 Fn. 176, S. 463.

178 Fn. 176, S. 463.

${ }^{179}$ So bezeichnete der BGH die Werberichtlinien des Verbandes der Zigarettenindustrie aus dem Jahr 1980, die auf vertraglicher Grundlage die Pflicht zum Abdruck eines Warnhinweises in der Anzeigenwerbung regeln, in seinem Leitsatz zum Urteil v. 25.11.1993 - I ZR 259/91 (KG) (Warnhinweis) in NJW 1994, $730 \mathrm{ff}$. als Ausdruck einer allgemeinen sittlichen Verpflichtung. Für weitere Beispiele vgl. Sosnitza in: MünchKommUWG, Grundl, Rn. 55 ff.

180 Sosnitza in FS für Bechtold, S. 516.

181 Novellierung des UWG im Jahr 2004.

182 Urteil des BGH v. 7. 2. 2006 - KZR 33/04 (OLG Hamburg) (Probeabonnement) in NJW 2006, $2627 \mathrm{ff}$.

${ }^{183}$ Leitsatz 2 der Probeabonnement-Entscheidung, vgl. Fn. 182.

${ }^{184}$ Urteil des BGH v. 7. 2. 2006 - KZR 33/04 (OLG Hamburg) (Probeabonnement), Tz. 21 in NJW 2006, $2627 \mathrm{ff}$.
} 
mit dieser Entscheidung zudem die Indizwirkung von Wettbewerbsregeln deutlich $\mathrm{ab}$, indem er ihnen nunmehr ,allenfalls eine indizielle Bedeutung für die Frage der Unlauterkeit" zugestand. ${ }^{185}$ Hintergrund dieser Verschärfung war ein grundsätzlicher Wandel bei der Beurteilung der Lauterkeit. So wurde unter Geltung des UWG 1909 maßgeblich auf das Anstandsgefühl der billig und gerecht Denkenden abgestellt wurde, da \1 UWG 1909 für die Unzulässigkeit von Handlungen darauf abstellte, ob diese ,gegen die guten Sitten verstoßen“". Als im Rahmen der Novellierung zum UWG 2004 der Begriff der guten Sitten durch den Begriff der Unlauterkeit ersetzt wurde, ${ }^{186}$ entstand Einigkeit darüber, ,dass der Wettbewerb in bedenklicher Weise beschränkt würde, wenn das Übliche zur Norm erhoben würde. "187 In der Literatur 188 wurden es jedoch — unter Anerkennung der vorstehend dargestellten Rechtslage — weiterhin grundsätzlich für möglich erachtet, Verstöße gegen Verhaltenskodizes als unlauter zu klassifizieren, wenn besondere zusätzliche Voraussetzungen erfüllt werden. Entsprechende Sachverhalte wurden jedoch keiner gerichtlichen Klärung unterzogen.

\section{B. Unter dem UWG 2008}

Grundsätzlich geändert hat sich die Gesetzeslage im Zusammenhang mit Verhaltenskodizes durch das am 22.12.2008 vom Deutschen Bundestag beschlossene „Erste Gesetz zur Änderung des Gesetzes gegen den unlauteren Wettbewerb”. 189 In diesem Zusammenhang wurde der Begriff des Verhaltenskodexes legaldefiniert ${ }^{190}$ und Spezialregelungen zu geschäftlichen Handlungen im Zusammenhang mit Verhaltenskodizes aufgenommen. ${ }^{191}$ Hintergrund war jedoch weniger ein eigenes Interesse des bundesdeutschen Gesetzgebers an Verhaltenskodizes ${ }^{192}$, sondern vielmehr die Verpflichtung zur Umsetzung der UGP-Richtlinie in deutsches Recht. ${ }^{193}$

\footnotetext{
185 Vgl. Fn. 182, S. 2629.

186 Begründung der Bundesregierung für den Gesetzentwurf eines Gesetzes gegen den unlauteren Wettbewerb vom 22.08.2003, BT.-Drs. 15/1487, S. 16.

187 Urteil des BGH v. 7. 2. 2006 - KZR 33/04 (OLG Hamburg) (Probeabonnement), Tz. 19 in NJW 2006, 2627 mit weiteren Nennungen.

188 Kocher, GRUR 2005, 647, 651 f., Kocher, Rechtliche Instrumente, insbesondere zur Durchsetzung und Umsetzung von Codes of Conduct im deutschen Recht, in Köpke/Röhr, S. 150 ff.

${ }^{189}$ Die in diesem Zuge eingeführten Regelungen zu Verhaltenskodizes bestehen in der aktuellen Fassung des UWG unverändert fort.

${ }^{190}$ Hierzu vertieft unter Kapitel 3.

${ }^{191}$ Hierzu vertieft unter Kapitel 5.

192 Vgl. „Punkt A. Problem und Ziel“ des Gesetzentwurfes der Bundesregierung, BT-Drs. 16/10145 unter Verweis auf den in Art. 19 UGP-Richtlinie benannten Umsetzungszeitpunkt.

193 Art. 249 Abs. 3 EG-Vertrag verpflichtet die Mitgliedstaaten zwar nicht zur direkten Übernahme europäischer Richtlinien in nationales Recht, jedoch sind die Richtlinien hinsichtlich des zu erreichenden Ziels verbindlich. Den Mitgliedstaaten bleibt es lediglich überlassen, die Form und
} 
Dies zeigt sich insbesondere in der Begründung des Gesetzentwurfes der Bundesregierung, wo an den entsprechenden Stellen oftmals lediglich auf die entsprechenden Regelungen der UGP-Richtlinie verwiesen wird, ${ }^{194}$ was in der Literatur unter anderem als ,lustlos" kritisiert wurde. 195

\section{Europäischer Ursprung der Regelungen zu Verhaltenskodizes im UWG}

Aufgrund dieses Ursprungs der Regelungen zu Verhaltenskodizes im UWG aus der UGP-Richtlinie sowie der mit dieser von den Richtliniengebern ${ }^{196}$ angestrebten Vollharmonisierung sind die UGP-Richtlinie sowie ihre Regelungen zu Verhaltenskodizes von herausragender Bedeutung für das Verständnis und die Auslegung des UWG im Allgemeinen und der dortigen Regelungen zu Verhaltenskodizes im Besonderen.

\section{Grundsätzliches zur UGP-Richtlinie}

a. Historie, Anwendungsbereich und Ziel

Die UGP-Richtlinie geht zurück auf das am 02.10.2001 vorgelegte Grünbuch Verbraucherschutz ${ }^{197}$, in welchem sich die Europäische Kommission für eine Förderung von der Selbst- und Ko-Regulierung ausgesprochen hat. ${ }^{198}$ Auf dieses Grünbuch aufbauend legte die Generaldirektion Gesundheit und Verbraucher der Europäischen Kommission einen Vorschlag für eine UGP-Richtlinie vor. ${ }^{199}$ Dieser setzte sich gegen einen ungefähr zeitgleich vorgelegten Vorschlag 200 der traditionell für das Lauterkeitsrecht zuständigen Generaldirektion Binnenmarkt durch und mün-

die Mittel zur Umsetzung zu wählen. Die hierfür angesetzte Frist lief im Falle der UGPRichtlinie am 12.06.2007 ab, Art. 19 UGP-Richtlinie.

194 So beispielsweise auf S. 12, S. 24 des Gesetzentwurfes der Bundesregierung, BT-Drs. 16/10145.

195 Birk, GRUR 2011, 196, 199.

196 Richtliniengeber sind das Europäische Parlament sowie der Rat der Europäischen Union, die Kommission unterbreitet formal lediglich einen Vorschlag.

${ }^{197} \mathrm{Zu}$ dessen Hintergrund und zur vertieften Historie der UGP-Richtlinie siehe Glöckner in: HarteBavendamm/Henning-Bodewig, UWG, Einl. B., Rn. 220 ff.

198 Grünbuch über den Verbraucherschutz in der Europäischen Union, $\operatorname{KOM}(2001), 531$ endgültig, S. 16 f., Ziffer 4.4 .

${ }^{199} \mathrm{KOM}(2003) 356$ endgültig.

200 Die Generaldirektion Binnenmarkt legte 2001 einen Vorschlag für eine neue Verordnung über Sales Promotion vor (ergänzte Fassung vom 25.10.2002, KOM (2002) 585 endg.). Der Vorschlag wurde jedoch nicht weiter verfolgt und galt zunächst als ,pending“, d.h. er sollte weiterhin geprüft werden, bevor er von der Kommission am 27. September 2005 - und somit nach dem Erlass der UGP-Richtlinie am 11. Mai 2005 — zurückgezogen wurde (KOM (2005) 462 endg.) 
dete in der am 11.05.2005 vom Europäischen Parlament und Rat als Richtlinie 2005/29/EG verabschiedeten UGP-Richtlinie. ${ }^{201}$

Der Anwendungsbereich der UGP-Richtlinie ist wie die Vorschlags- und Regelungskompetenz der federführenden Generaldirektion Gesundheit und Verbraucherschutz auf geschäftliche Handlungen von Unternehmern gegenüber Verbrauchern, den sogenannten b2c ${ }^{202}$-Bereich, beschränkt. Geschäftliche Handlungen zwischen Unternehmern werden von ihr nicht erfasst. ${ }^{203}$ Die UGP-Richtlinie schützt nach ihrem Erwägungsgrund 8 aber mittelbar auch die rechtmäßig handelnden Unternehmer vor Mitbewerbern, die sich nicht an Regelungen der UGPRichtlinie (bei Ihren geschäftlichen Handlungen gegenüber Verbrauchern) halten. Eine Erweiterung des Anwendungsbereiches auf geschäftliche Handlungen zwischen Unternehmern, den sogenannten b2 b 204 -Bereich, wäre aufgrund der beschränkten Kompetenz der Generaldirektion Gesundheit und Verbraucher innerhalb der Europäischen Kommission nur unter Mitwirkung der Generaldirektion Binnenmarkt zu erreichen gewesen. ${ }^{205}$ Die dahingehend gehegte Hoffnung, die beiden Vorschläge würden sich ergänzen, erwies sich jedoch als Illusion. ${ }^{206}$ Somit stellt die UGP-Richtlinie zwar unbestreitbar das Herzstück ${ }^{207}$ der mit Beginn der 1980 er Jahre begonnenen Vereinheitlichung des europäischen Lauterkeitsrechts ${ }^{208}$ dar. Sie führt aber gleichzeitig zu einer Aufspaltung der Bereiche b2c und b2b, die zuvor einheitlich geregelt waren ${ }^{209}$, was im deutschsprachigen Raum einhellig

201 Die Richtlinie wurde am 11. Juni 2005 im Amtsblatt veröffentlicht und trat am 12. Juni 2005 in Kraft.

202 Business to Consumer.

${ }^{203}$ Die UGP-Richtlinie ,erfasst und berübrt nicht die nationalen Rechtsvorscbriften in Bezug auf unlautere Geschäftspraktiken, die lediglich die wirtschaftlichen Interessen von Mitbewerbern schädigen oder sich auf ein Rechtsgeschäft zwischen Gewerbetreibenden beziehen ", Erwägungsgrund 6 der UGP-Richtlinie.

204 Business to Business.

205 Wiebe, JBl. 2007, 69, 71.

${ }^{206}$ Henning-Bodewig, GRUR Int. 2005, 629, 629.

207 Hoeren, BB 2008, 1182.

${ }^{208}$ Die erste Harmonisierung erfolgte mit der Richtlinie 84/450/EWG zur Angleichung der Rechtsund Verwaltungsvorschriften der Mitgliedstaaten über irreführende Werbung, welche durch die Richtlinie 97/55/EG auf den Bereich der vergleichenden Werbung erweitert wurde. Ihr folgten verschiedene lauterkeitsrechtliche Regelungen, die jedoch zumeist in Richtlinien über andere Rechtsbereiche enthalten waren. So bspw. in der E-Commerce-Richtlinie 2000/31/EG oder in der Richtlinie 2002/58/EG über die Verarbeitung personenbezogener Daten. Darüber hinaus entstanden Richtlinien, die das Lauterkeitsrecht in bestimmten Sektoren betrafen wie bspw. die Richtlinie 2003/22/EG über die Angleichung der Rechts- und Verwaltungsvorschriften über Werbung und Sponsoring zugunsten von Tabakerzeugnissen.

209 So enthielt die Richtlinie 84/450/EWG zur Angleichung der Rechts- und Verwaltungsvorschriften der Mitgliedstaaten über irreführende Werbung bereits in ihrer Grundfassung im Jahr 1984 in ihrem Art. 1 die sogenannte Schutzzwecktrias (,Zweck dieser Richtlinie ist der Schutz der Verbraucher, der Personen, die einen Handel oder ein Gewerbe betreiben oder ein Handwerk oder einen freien Beruf ausüben, sowie der Interessen der Allgemeinheit gegen irrefübrende Werbung und deren unlautere Auswirkungen."), welche erst im Jahr 2004 in das UWG aufgenommen wurde. Diese umfassende Schutzbestim- 
kritisiert wurde. 210 Darüber hinaus haben die Richtliniengeber den Anwendungsbereich der UGP-Richtlinie auch innerhalb des b2c-Bereichs beschränkt. So ist die UGP-Richtlinie gegenüber anderen gemeinschaftsrechtlichen Regelungen subsidiär ${ }^{211}$, verzichtet auf Regelungen für das Vertragsrecht ${ }^{212}$, die Zuständigkeit von Gerichten ${ }^{213}$ sowie für Berufsregelungen reglementierter Berufe ${ }^{214}$ und enthält Ausnahmen für die abweichende Regelungen der Mitgliedstaaten in Bezug auf die Gesundheits- und Sicherheitsaspekte von Produkten. ${ }^{215}$

Innerhalb ihres Anwendungsbereiches verfolgt die UGP-Richtlinie das Ziel, das europäische Lauterkeitsrecht zu vereinheitlichen und einen das gesamte Lauterkeitsrecht umfassenden Rechtsrahmen zu schaffen. ${ }^{216}$ Die Richtlinie führt somit zur Vollharmonisierung 217 des europäischen Lauterkeitsrechts in dem von ihr betroffenen Bereich, so dass die von der UGP-Richtlinie aufgestellten Standards von den Mitgliedstaaten weder über- noch unterschritten werden dürfen.218 Dies gilt jedoch erst seit dem 12.06.2013, da Art. 3 Abs. 5 UGP-Richtlinie den Mitgliedstaaten unter bestimmten Voraussetzungen gestattete, bis zu diesem Zeitpunkt nationale Vorschriften beizubehalten, ,die restriktiver oder strenger sind als diese Ricbtlinie und zur Umsetzung von Richtlinien erlassen wurden und die Klauseln viber eine Mindestangleichung enthalten."

b. Aufbau und Systematik

Entsprechend dem üblichen Aufbau von europäischen Richtlinien enthält Art. 1 den Zweck der Richtlinie. ${ }^{219}$ Art. 2 definiert die wesentlichen Begriffe der Richtlinie, wobei im Hinblick auf den eingeschränkten Anwendungsbereich ${ }^{220}$ die Defi-

mung wurde mit Art. 14 der UGP-Richtlinie dahingehend verkürzt, dass nunmehr nur noch der Schutz von Gewerbetreibenden umfasst ist.

210 Wiebe, JBl. 2007, 69, 71.

211 Art. 3 Abs. 4 UGP-Richtlinie: „Kollidieren die Bestimmungen dieser Ricbtlinie mit anderen Rechtsvorschriften der Gemeinschaft, die besondere Aspekte unlauterer Geschäftspraktiken regeln, so gehen die Letzteren vor und sind für diese besonderen Aspekte maßgebend."

212 Art. 3 Abs. 2 UGP-Richtlinie.

213 Art. 3 Abs. 7 UGP-Richtlinie.

214 Art. 3 Abs. 8 UGP-Richtlinie.

215 Art. 3 Abs. 3 UGP-Richtlinie.

${ }^{216}$ Erwägungsgrund 6 der UGP-Richtlinie, Art. 1 UGP-Richtlinie: „Zweck der Richtlinie ist es, durch Angleichung der Rechts- und Verwaltungsvorschriften der Mitgliedstaaten über unlautere Geschäftspraktiken, die die wirtschaftlichen Interessen der Verbraucher beeinträchtigen, zu einem reibungslosen Funktionieren des Binnenmarktes und zum Erreichen eines hohen Verbraucherschutzniveaus beizutragen."

${ }^{217}$ Erwägungsgrund 13 der UGP-Richtlinie: „,\{...\}. Das durch diese Richtlinie eingeführte einzige, gemeinsame generelle Verbot $\{\ldots\}$.."

218 Nordemann, Rn. 17.

219 Zum Inhalt von Art. 1 vgl. Fn. 216.

${ }^{220}$ Vgl. Kapitel 2B.I.1.a. 
nitionen des Verbrauchers ${ }^{221}$ und des Gewerbetreibenden 222 von besonderer Bedeutung sind. Darüber hinaus wird auch der Begriff des Verhaltenskodexes definiert. ${ }^{223}$ Art. 3 regelt den Anwendungsbereich der UGP-Richtlinie. ${ }^{224}$ Die sogenannte Binnenmarktklausel in Art. 4 enthält die Überreste des Versuchs der Kommission, der UGP-Richtlinie kollisionsrechtlich das Herkunftslandprinzip zugrunde zulegen. ${ }^{225} \mathrm{Im}$ Anschluss daran bestimmten die Artikel 5 bis 9 auf der Grundlage des Verbots unlauterer Geschäftspraktiken ${ }^{226}$, welche Geschäftspraktiken als unlauter gelten. Dies ist zunächst jede Geschäftspraktik, die „,den Erfordernissen der beruflichen Sorgfaltspflicht227 widerspricht“228 und „,das wirtschaftliche Verhalten des Durchschnittsverbrauchers ${ }^{22}\{\ldots\}$ wesentlich beeinflusst oder dazu geeignet ist, es wesentlich zu beeinflussen". ${ }^{230}$ Dabei müssen beide Tatbestandsvoraussetzungen kumulativ vorliegen. Als besondere Beispielsfälle der Unlauterkeit („,insbesondere $e^{6}$ ) gelten irreführende und aggressive Geschäftspraktiken, ${ }^{231}$ die in den Artikeln 6 und 7 (irreführende Handlungen und Unterlassungen) sowie den Artikeln 8 und 9 (aggressive Geschäftspraktiken) konkretisiert werden. Dabei setzt die UGP-Richtlinie jeweils voraus, dass der von der jeweiligen Geschäftspraktik betroffene Durchschnittsverbraucher durch diese zu einer geschäftlichen Entscheidung ${ }^{232}$ veranlasst wird, die er ansonsten nicht getroffen hätte, oder dass die Geschäftspraktik grundsätzlich geeignet ist, ihn zu einer geschäftlichen Entscheidung zu veranlassen. Diese Voraussetzung gilt jedoch nicht für die in Anhang I der UGP-Richtlinie aufgeführten irreführenden (Nr. 1 bis $\mathrm{Nr}$. 23) und aggressiven (Nr. 24 bis 31) Geschäftspraktiken. Denn diese sind nach Art. 5 Abs. 5 UGP-Richtlinie ,unter allen

221 Art. 2 lit a) UGP-Richtlinie.

222 Art. 2 lit b) UGP-Richtlinie.

223 Art. 2 lit f) UGP-Richtlinie.

224 Vgl. Kapitel 2B.I.1.a.

225 Vgl. Kapitel 4B.

226 Durch den als Generalklausel bezeichneten Art. 5 Abs. 1 UGP-Richtlinie: „Unlautere Geschäftspraktiken sind verboten."

227 Die berufliche Sorgfalt wird wiederum von Art. 2 lit h) UGP-Richtlinie als „der Standard an Fachkenntnissen und Sorgfalt, bei denen billigerweise davon ausgegangen werden kann, dass der Gewerbetreibende sie gegenüber dem Verbraucher gemäß den anständigen Marktgepflogenheiten und/ oder dem allgemeinen Grundsatz von Treu und Glauben in seinem Tätigkeitsbereich anwendet" definiert.

228 Art. 5 Abs. 2 lit. a) UGP-Richtlinie.

${ }^{229}$ Der Durchschnittverbraucher ist jedoch nur solange als Maßstab anzusetzen, als die Geschäftspraktik nicht in für den Gewerbetreibenden vorhersehbarer Art und Weise nur eine besonders schutzbedürftige Gruppe (bspw. aufgrund ihres Alters, geistiger oder körperlicher Gebrechen) beeinflusst. In diesen Fällen ist auf die Perspektive eines durchschnittlichen Mitglieds dieser Gruppe abzustellen, Art. 5 Abs. 3 UGP-Richtlinie.

230 Art. 5 Abs. 2 lit. b) UGP-Richtlinie.

231 Art. 5 Abs. 4 UGP-Richtlinie.

232 Art. 2 lit. k) UGP-Richtlinie: ,geschäftliche Entscheidung “ jede Entscheidung eines Verbraucher darüber, ob, wie und unter welchen Bedingungen er einen Kauf tätigen, eine Zablung insgesamt oder teilweise leisten, ein Produkt behalten oder abgeben oder ein vertragliches Recht im Zusammenhang mit dem Produkt ausïben will, unabhängig davon, ob der Verbraucher beschließt, tätig zu werden oder ein Tätigwerden zu unterlassen." 
Die Bedeutung von Verhaltenskodizes im UWG

Umständen als unlauter anzusehen." An dieses Herzstück der UGP-Richtlinie schlieBen sich ein allein aus dem Art. 10 bestehendes Kapitel 3 über Verhaltenskodizes $^{233}$ sowie die Schlussbestimmungen an. Letztere enthalten neben Regelungen über die Verpflichtungen der Mitgliedstaaten zur Implementierung von wirksamen und geeigneten Mitteln zur Bekämpfung des unlauteren Wettbewerbs ${ }^{234}$, zur Festlegung von wirksamen, verhältnismäßigen und abschreckenden Sanktionen ${ }^{235}$ und zur Information der Verbraucher über die Umsetzungsmaßnahmen der Richtlinie ${ }^{236}$, die mit der UGP-Richtlinie beschlossenen Änderungen bereits bestehender Richtlinien ${ }^{237}$ sowie zum weiteren Umgang mit der Richtlinie ${ }^{238}$, ihrer Umsetzung $^{239}$, ihrem Inkrafttreten ${ }^{240}$ und ihren Adressaten. ${ }^{241}$ Den Abschluss der UGPRichtlinie bilden der bereits benannte Anhang I mit der Auflistung der in jedem Fall unlauteren Geschäftspraktiken sowie der Anhang II. Letzterer enthält eine nicht abschließende Auflistung der im Gemeinschaftsrecht festgelegten Informationspflichten, deren Vorenthalten im Rahmen der Irreführung durch Unterlassen von Bedeutung ist. ${ }^{242}$

\section{Verbaltenskodizes in der UGP-Richtlinie}

a. Bedeutung der Selbstregulierung in der Gesetzgebungshistorie der UGPRichtlinie

Die Förderung der Selbstregulierung war ein wesentlicher Bestandteil der im Grünbuch Verbraucherschutz ${ }^{243}$ dargelegten Überlegungen der Europäischen Kommission zur Neuausrichtung des Verbraucherschutzes. ${ }^{244}$ Denn die Europäische Kommission sah die Selbstregulierung aufbauend auf ihrem ebenfalls 2001 veröffentlichten Weißbuch Europä̈sches Regieren ${ }^{245}$ als Möglichkeit an, ihre Verbraucherschutzziele bei gleichzeitiger Verminderung des Rechtssetzungs- und KoRegulierungsbedarfs zu erreichen. ${ }^{246}$ Als Voraussetzung für eine wirksame Selbst-

\footnotetext{
233 Vgl. hierzu sogleich nachfolgend Kapitel 2B.I.2.

234 Art. 11 und 12 UGP-Richtlinie.

235 Art. 13 UGP-Richtlinie.

236 Art. 17 UGP-Richtlinie.

237 Art. 14 bis 16 UGP-Richtlinie.

238 Art. 18 UGP-Richtlinie.

239 Art. 19 UGP-Richtlinie.

240 Art. 20 UGP-Richtlinie.

241 Art. 21 UGP-Richtlinie.

242 Art. 7 Abs. 5 und Erwägungsgrund 15 UGP-Richtlinie, vgl. Kapitel 6C.II.1.b.

243 Auf welches die UGP-Richtlinie zurückgeht, vgl. Kapitel 2B.I.1.a.

244 Wiebe, JBl. 2007, 69, 70, Grünbuch zum Verbraucherschutz in der Europäischen Union, KOM (2001) 0531 endgültig.

245 Weißbuch Europäisches Regieren, KOM (2001) 428 endgültig, S. 26 ff.

246 Grünbuch zum Verbraucherschutz in der Europäischen Union, KOM (2001) 0531 endgültig, S. 16, Ziffer 4.4 .
} 
regulierung erachtete das Grünbuch Verbraucherschutz dabei, dass ,,\{...\} die Nichterfüllung einer gegenüber einem Verbraucher freiwillig eingegangenen Verpflichtung durch ein Unternehmen als eine irrefübrende oder unlautere Geschäftspraxis definiert" 247 wird, so dass der Verstoß gegen solche freiwilligen Verpflichtungen eine Sanktionierung aufgrund des Lauterkeitsrecht nach sich zieht. Diese Forderung nach einer staatlichen Sanktionierung von Verstößen gegen Verhaltenskodizes anstelle einer Selbstkontrolle begründete die Europäische Kommission in ihrer „Mitteilung über Folgemaß-

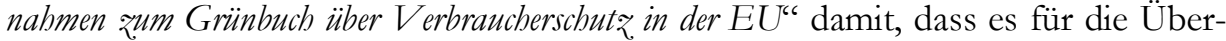
zeugung von Mitgliedstaaten, in welchen Verhaltenskodizes nicht üblich sind, wesentlich sei, sicherzustellen, dass Verpflichtungen aus Verhaltenskodizes auch eingehalten würden. ${ }^{248}$

Im Rahmen der Konsultationen zur Vorbereitung der UGP-Richtlinie wurde dementsprechend ein besonderes Augenmerk auf diejenigen Mitgliedstaaten gerichtet, die statt auf eine umfassende gesetzliche Regelung des Lauterkeitsrechts auf eine Selbstregulierung gesetzt hatten. So waren beispielsweise der Fair Trading Act im Vereinigten Königreich und der Stichting Reclame Code in den Niederlanden elementarer Bestandteil des Lauterkeitsrechts, da es dort keine gesetzliche Regulierung des lauteren Wettbewerbes ${ }^{249}$ beziehungsweise kein eigenes Lauterkeitsrecht mit Spezialregelungen ${ }^{250}$ gab. Entsprechende Verhaltenskodizes wurden von Organisationen und Verbänden der Wirtschaft oder sogar gemeinsam durch staatliche und private Organisationen (z. B. in Dänemark) aufgestellt ${ }^{251}$ und von diesen auch durchgesetzt. ${ }^{252}$

Diese Vorstellungen der Europäischen Kommission aus dem Grünbuch Verbraucherschutz, Verhaltenskodizes über das Konzept der Ko-Regulierung fest in das System des europäischen Lauterkeitsrechts aufzunehmen, konnten sich jedoch aufgrund erheblicher Kritik insbesondere aus den Mitgliedstaaten ohne eine Tradition der Selbstregulierung im Lauterkeitsrecht wie insbesondere Deutschland ${ }^{253}$

247 Grünbuch zum Verbraucherschutz in der Europäischen Union, KOM (2001) 0531 endgültig, S. 16, Ziffer 4.4. Als zweites entscheidendes Element für eine wirksame Selbstregulierung machte das Grünbuch Verbraucherschutz aus, dass sich die Regelungen „nicht nur auf Unternebmen erstrecken, die mit Verbrauchern Handel treiben, sondern auch auf Unternehmensverbände und andere Organisationen, die Empfehlungen ₹u Geschäftspraktiken ausgesprochen und Regeln etc. aufgestellt haben." Grünbuch zum Verbraucherschutz in der Europäischen Union, KOM (2001) 0531 endgültig, S. 17, Ziffer 4.4 .

248 KOM (2002) 289 endgültig, S. 13, Tz. 29.

${ }^{249}$ Im Vereinigten Königreich, vgl. Schulze/Schulte-Nölke, S. 8.

${ }^{250}$ In den Niederlanden wurden die Grundzüge des Wettbewerbsrechts aus der zentralen Vorschrift des Deliktsrechts abgeleitet und von Einzelregelungen ergänzt, die über vielerlei Gesetze verstreut waren, vgl. Schulze/Schulte-Nölke, S. 12.

251 Schulze/Schulte-Nölke, S. 18.

${ }^{252}$ Für die Niederlande erfolgt dies durch die Reclame Code Commissie und das College van Beroep, vgl. Schulze/Schulte-Nölke, S. 20 f.

253 Zum Inhalt der Kritik Wiebe, WRP 2002, 283, 288 ff. 
nicht durchsetzen. ${ }^{254}$ So griff bereits der Entwurf der UGP-Richtlinie, für welchen sich das System eines Spezialgesetzes mit lauterkeitsrechtlicher Generalklausel durchgesetzt hatte ${ }^{255}$, den Ansatz der Selbstregulierung nur insoweit auf, als er Kontrolle von Verhaltenskodizes durch deren Urheber neben den eigentlichen Gerichts- und Verwaltungsverfahren nicht ausschloss. ${ }^{256}$ In der verabschiedeten Fassung der UGP-Richtlinie fanden sich die Vorstellungen der Europäischen Kommission dann in noch rudimentärerer Form wieder. ${ }^{257}$ So wurde der Begriff der Kodizes auf Gemeinschaftsebene, dessen Aufnahme noch als Zeichen der besonderen Wertschätzung von Verhaltenskodizes eingeordnet wurde ${ }^{258}$, aus Art. 10 gestrichen. Stattdessen wurde in diesem noch einmal besonders hervorgehoben, dass die Kontrolle durch die Urheber der Verhaltenskodizes keinen Verzicht auf die Rechtsbehelfe vor einem Gericht oder einer Verwaltungsbehörde bedeutet. Durchgesetzt hat sich die Europäische Kommission dahingegen insoweit, als materielle Regelungen zur Unlauterkeit von Verhaltensweisen im Zusammenhang mit Verhaltenskodizes in die UGP-Richtlinie aufgenommen wurden.

b. Regelungen zur Verhaltenskodizes in der UGP-Richtlinie

Die UGP-Richtlinie enthält neben den Definitionen der Begriffe Verhaltenskodex 259 und Urheber eines Kodex ${ }^{260}$ im materiellen Teil drei Regelungen zur Unlauterkeit von Geschäftspraktiken im Zusammenhang mit Verhaltenskodizes. So betrifft die Nr. 1 des Anhangs I zur UGP-Richtlinie die „Behauptung eines Gewerbetreibenden, zu den Unterzeichnern eines Verhaltenskodexes zu gehören, obgleich dies nicht der Fall ist." Eine solche Behauptung ist nach Art. 5 Abs. 5 UGP-Richtlinie in jedem Fall unlauter und damit gemäß Art. 5 Abs. 1 UGP-Richtlinie verboten. Gleiches gilt nach Nr. 3 des Anhangs I für die „Behauptung, ein Verhaltenskodex sei von einer öffentlichen oder einer anderen Stelle gebilligt, obgleich dies nicht der Fall ist. "261 Dass sich in der Auflistung in

${ }^{254}$ Micklitz in: MünchKommUWG, EG E, Rn. 196, Wiebe, JBl. 2007, 69, 70.

255 Art. 5 Abs. 1 des Vorschlags der Kommission für die UGP-Richtlinie, KOM (2003) 356 endgültig.

256 Art. 10 des Vorschlags der Kommission für die UGP-Richtlinie, KOM (2003) 356 endgültig.

${ }^{257}$ Micklitz in: MünchKommUWG, EG D, Rn. 70, 142 ff.

258 Köhler/Lettl, WRP 2003, 1019, 1047 f.

259 Art. 2 lit f) UGP-Richtlinie: „,,Verbaltenskodex“ eine Vereinbarung oder ein Vorschriftenkatalog, die bæw. der nicht durch die Rechts- und Verwaltungsvorschriften eines Mitgliedstaates vorgeschrieben ist und das Verbalten der Gewerbetreibenden definiert, die sich in Bezug auf eine oder mehrere Geschäftspraktiken oder Wirtschafts zweige auf diesen Kodex verpflichten."

260 Art. 2 lit g) UGP-Richtlinie: „„,Urheber eines Kodex“ jede Rechtspersönlichkeit, einschließlich einzelner Gewerbetreibender oder Gruppen von Gewerbetreibenden, die für die Formulierung und Überarbeitung eines Verbaltenskodex und/ oder für die Überwachung der Einhaltung dies Kodexes durch alle diejenigen, die sich darauf verpflichtet haben, zuständig ist".

${ }^{261}$ Eine genauere Untersuchung der Bedeutung und Reichweite dieser Regelungen erfolgt im Rahmen der Untersuchung ihrer Umsetzung in deutsches Recht, vgl. hierzu Kapitel 5A. 
Anhang I zwei Geschäftspraktiken im Zusammenhang mit Verhaltenskodizes an die Nummern eins und drei befinden, macht die Bedeutung deutlich, welche die Richtliniengeber diesem Regelungsbereich zugemessen haben. ${ }^{262}$ Die dritte Regelung zu Geschäftspraktiken im Zusammenhang mit Verhaltenskodizes findet sich in Art. 6 Abs. 2 lit. b) ${ }^{263}$ UGP-Richtlinie. Sie setzt den Willen der Richtliniengeber ${ }^{264}$ um, die Nichteinhaltung von durch Unternehmen gegenüber Verbrauchern freiwillig eingegangenen Verpflichtungen zu sanktionieren. Diese Regelung führt zur Irreführung und damit zur Unlauterkeit von Geschäftspraktiken, welche „,die Nichteinhaltung von Verpflichtungen, die der Gewerbetreibende im Rabmen von Verbaltenskodizes, auf die er sich verpflichtet hat, eingegangen ist, $\{\ldots\}$ " enthalten. Voraussetzung ist jedoch, dass es sich bei dem Verhaltenskodex ,nicht um eine Absichtserklärung, sondern um eine eindeutige Verpflichtung handelt, deren Einhaltung nachprüfbar ist, ${ }^{265}$ und der Gewerbetreibende im Rahmen einer Geschäftspraxis darauf binweist, dass er durch den Kodex gebunden ist."266 Wie im Rahmen der Irreführung üblich, ist eine solche Geschäftspraktik allerdings nur dann auch irreführend, ,wenn sie im konkreten Fall unter Berücksichtigung aller tatsächlichen Umstände einen Durchschnittsverbraucher zu einer geschäftlichen Entscheidung veranlasst oder zu veranlassen geeignet ist, die er ansonsten nicht getroffen bätte. “267

Neben diesen die Unlauterkeit von Geschäftspraktiken im Zusammenhang mit Verhaltenskodizes betreffenden Regelungen enthält die UGP-Richtlinie als Überbleibsel der Bestrebungen der Europäischen Kommission zur konzeptionellen Einbindung der Ko-Regulierung in die UGP-Richtlinie das aus dem Art. 10 bestehendes Kapitel 3. Dabei ist sowohl das Kapitel als auch der Artikel mit „Verhaltenskodizes" überschrieben. ${ }^{268}$ Art. 10 UGP-Richtlinie stellt klar, dass die UGPRichtlinie die Kontrolle unlauterer Geschäftspraktiken durch die Urheber von Kodizes sowie die Inanspruchnahme solcher Einrichtungen durch Dritte nicht ausschließt, sofern gewährleistet ist, dass parallel auch Gerichts- oder Verwaltungsverfahren hoheitlicher Natur zur Verfügung stehen und die Inanspruchnahme der privaten Kontrolleinrichtungen keinen Verzicht auf einen Rechtsbehelf gegenüber Gericht oder Verwaltungsbehörde bedeutet. ${ }^{269}$ Auch wenn sich die Richtliniengeber von dieser ausdrücklichen Gestattung der Selbstkontrolle eine

\footnotetext{
262 In dem ersten Entwurf der UGP-Richtlinie waren diese beiden Geschäftspraktiken sogar an Nr. 1 und Nr. 2 des Anhangs I gesetzt, KOM (2003), 356 endgültig.

${ }^{263}$ Die Norm wurde im UWG in $\ 5$ Abs. 1 S. 2 Nr. 6 umgesetzt, vgl. Begründung der Bundesregierung für den Gesetzentwurf zur Änderung des UWG vom 20.08.2008, BT.-Drs. 16/10145, S. 17.

264 Vgl. Kapitel 2B.I.2.a.

265 Art. 6 Abs. 2 lit b) i) UGP-Richtlinie.

266 Art. 6 Abs. 2 lit b) ii) UGP-Richtlinie.

267 Art. 6 Abs. 2 UGP-Richtlinie.

268 Vgl. Kapitel 2B.I.2.a.

${ }^{269}$ KOM (2003), 356 endgültig, Punkt 75 der Begründung.
} 
Entlastung der Verwaltungsbehörden und Gerichte versprachen ${ }^{270}$, kommt doch durch die zurückhaltende Formulierung („Die Richtlinie schließt $\{\ldots\}$ nicht aus $\{\ldots\}$. .) zum Ausdruck, dass sich die Richtliniengeber mit ihrem Bestreben nach einer Institutionalisierung der Selbstregulierung nicht durchsetzen konnten. ${ }^{271}$ Insofern kommt Art. 10 UGP-Richtlinie im wesentlichen symbolische Bedeutung zu, indem er die in Mitgliedstaaten mit einer entsprechenden lauterkeitsrechtlichen Tradition gelebte Praxis der Selbstkontrolle ausdrücklich weiterhin zulässt und damit die Kontrolle der Lauterkeit des Wettbewerbes zumindest teilweise weiterhin parallel $^{272}$ in die Selbstverwaltung der beteiligten Wirtschaftskreise stellt. ${ }^{273}$ Darüber hinaus sieht die UGP-Richtlinie in Art. 11 Abs. 1 Unterabsatz 4 lit. b) die Möglichkeit vor, dass sich die von der UGP-Richtlinie vorgesehenen Rechtsbehelfe auch gegen den Urheber eines Verhaltenskodexes richten können. Dies gilt jedoch nur für den Fall, dass der betreffende Kodex der Nichteinhaltung rechtlicher Vorschriften Vorschub leistet.

\section{Regelungen zu Verhaltenskodizes im UWG 2008}

Diese Regelungen zu Verhaltenskodizes aus der UGP-Richtlinie wurden vom deutschen Gesetzgeber nur teilweise umgesetzt. So wurde der Begriff des Verhaltenskodexes entsprechend der Vorgabe der UGP-Richtlinie legaldefiniert ${ }^{274}$ sowie die materiellen Spezialregelungen zu geschäftlichen Handlungen im Zusammenhang mit Verhaltenskodizes übernommen. ${ }^{275}$ Nicht in das UWG übernommen wurden dahingegen die Regelungen aus Art. 11 Abs. 1 Unterabsatz 4 lit. b) UGPRichtlinie sowie aus Art. 10 UGP-Richtlinie. Hinsichtlich Art. 10 UGP-Richtlinie begründete der deutsche Gesetzgeber seinen Verzicht auf gesetzgeberische Maßnahmen damit, dass das geltende Recht die von Art. 10 UGP-Richtlinie angesprochene Selbstkontrolle nicht ausschließe. Dem ist zuzustimmen, da das Lauterkeitsrecht dem Deliktsrecht zuzuordnen ist und es den Parteien damit wie bei jedem zivilrechtlichen Anspruch freisteht, die Angelegenheit außergerichtlich, wenn gewünscht unter Beiziehung eines unabhängigen Schiedsgerichts oder Mediators, beizulegen. Entsprechend hat der Gesetzgeber zurecht auf die bereits im UWG 2004 bestehende Regelung des \15 UWG verwiesen. Diese eröffnet die Möglichkeit, Einigungsstellen der Industrie- und Handelskammern anzurufen. ${ }^{276}$

\footnotetext{
270 Erwägungsgrund 20 der UGP-Richtlinie.

271 Vgl. Kapitel 2B.I.2.a.

272 Denn unabhängig davon werden alle Mitgliedstaaten verpflichtet, in jedem Fall eine Kontrolle des Lauterkeitsrechts durch Verwaltungsbehörden und Gerichte sicherzustellen.

273 Veelken, WRP 2004, S. 1, 27 f.

274 Hierzu vertieft unter Kapitel 3.

275 Hierzu vertieft unter Kapitel 5.

276 Begründung der Bundesregierung für den Gesetzentwurf zur Änderung des UWG vom 20.08.2008, BT.-Drs. 16/10145, S. 18.
} 
Seinen Verzicht auf die Umsetzung von Art. 11 Abs. 1 Unterabsatz 4 lit. b) UGPRichtlinie hat der deutsche Gesetzgeber dahingegen nicht begründet. Dies war jedoch auch nicht notwendig, da die UGP-Richtlinie es diesbezüglich ausdrücklich jedem Mitgliedstaat überlassen hat, zu entscheiden, ob er die Regelung in nationales Recht umsetzt. ${ }^{277} \mathrm{Da}$ der deutsche Gesetzgeber somit beide Regelungen der UGP-Richtlinie, welche den Begriff des Urhebers eines Kodex verwenden, nicht übernommen hat, wird dieser im UWG auch nicht verwendet. Entsprechend hat der deutsche Gesetzgeber auch auf seine Definition im Rahmen von $\int 2$ Abs. 1 UWG verzichtet. ${ }^{278}$ 20.08.2008, BT.-Drs. 16/10145, S. 12. 



\section{Kapitel 3}

\section{Der Begriff des Verhaltenskodexes i. S. d. UWG}

Zentrale Bedeutung bei der Beurteilung der mit dem UWG 2008 eingeführten Regelungen zur Unlauterkeit geschäftlicher Handlungen im Zusammenhang mit Verhaltenskodizes kommt der Frage zu, wann ein Verhaltenskodex i. S. d. UWG vorliegt. Ausgangspunkt hierfür ist die Definition in $\int 5$ Abs. 1 Nr. 5 UWG, welche sich an die Definition des Verhaltenskodexes in der UGP-Richtlinie ${ }^{279}$ anlehnt. ${ }^{280}$ Demnach sind Verhaltenskodizes

„Vereinbarungen oder Vorschriften über das Verhalten von Unternehmern, zu welchem diese sich im Bezug auf Wirtschaftszweige oder einzelne geschäftliche Handlungen ver-

${ }^{279}$ Art. 2 lit. f) UGP-Richtlinie: ,,,V Verbaltenskodex“ eine Vereinbarung oder ein Vorschriftenkatalog, die brw. der nicht durch die Rechts- oder Verwaltungsvorschriften eines Mitgliedstaates vorgeschrieben ist und das Verbalten der Gewerbetreibenden definiert, die sich in Bezug auf eine oder mebrere spezielle Geschäftspraktiken oder Wirtschaftsziveige auf diesen Kodex verpflichten."

280 Begründung der Bundesregierung für den Gesetzentwurf zur Änderung des UWG vom 20.08.2008, BT.-Drs. 16/10145, S. 21. Aufgrund fehlender anderslautender Hinweise ist im Rahmen der ohnehin richtlinienkonform vorzunehmenden Auslegung somit davon auszugehen, dass die marginalen Abweichungen zur Definition der UGP-Richtlinie allein redaktionelle Gründe hatten und keine inhaltliche Unterscheidung bewirken sollten. 
pflichtet haben, obne dass sich solche Verpflichtungen aus Gesetzes- oder Verwaltungsvorschriften ergeben." 281

Zwar ist das negative Tatbestandsmerkmal der Definition („obne dass sich solche Verpflichtungen aus Gesetzes- oder Verwaltungsvorscbriften ergeben."), aus welchem sich das nach dem allgemeinen Sprachgebrauch wesentliche Merkmal der Freiwilligkeit ergibt ${ }^{282}$, eindeutig. Jedoch ist der positiv formulierte Teil der Definition (,Vereinbarungen oder Vorschriften über das Verhalten von Unternehmern, zu welchem diese sich im Bezug auf Wirtschaftszweige oder einzelne geschäftliche Handlungen verpflichtet haben") so weit gefasst, dass diesbezüglich eine einschränkende Auslegung geboten ist. Dies gilt insbesondere für den inhaltlichen Gegenstand eines Verhaltenskodexes. ${ }^{283}$ Denn andernfalls wären auch Regelwerke als Verhaltenskodizes i. S. d. \2 Abs. 1 Nr. 5 UWG einzuordnen, die weder nach dem allgemeinen Sprachgebrauch ${ }^{284}$ noch nach dem Begriffsverständnis der Richtliniengeber mit einem Verhaltenskodex in Verbindung gebracht werden können. Unabhängig von der konkreten Ausgestaltung der Definition ist es in jedem Fall äußerst begrüßenswert, dass die Richtliniengeber den schwierigen Begriff des Verhaltenskodexes für das Lauterkeitsrecht definiert haben, nach dem der Begriff in früheren Richtlinien zwar verwendet ${ }^{285}$, aber nicht bestimmt wurde. ${ }^{286}$.

\section{A. Freiwilligkeit der Verpflichtung}

Als eindeutigste, weil gesondert negativ formulierte Voraussetzung eines Verhaltenskodexes kann der Definition des $\ 2$ Abs. 1 Nr. 5 UWG die Freiwilligkeit der Verpflichtung entnommen werden. Freiwilligkeit bedeutet in diesem Zusammenhang, dass das jeweilige Unternehmen nicht aufgrund eines Gesetzes oder einer Verwaltungsvorschrift auf das jeweilige Verhalten verpflichtet ist. Da der Begriff der Verwaltungsvorschrift aus der UGP-Richtlinie übernommen wurde, ist er an dieser Stelle nicht so zu verstehen, wie er in der deutschen Rechtsterminologie verwendet wird, sondern entsprechend dem Verständnis der Richtliniengeber. Denn nach der deutschen Rechtsterminologie sind Verwaltungsvorschriften, generell abstrakte Anordnungen einer Behörde an nachgeordnete Bebörden oder eines Vorgesetżten an die ihm unterstellten Verwaltungsbediensteten", welche entweder die innere Ordnung einer Behörde oder das sachliche Verwaltungshandeln betreffen. ${ }^{287}$ Als solche

\footnotetext{
${ }^{281}$ So die Legaldefinition in $₫ 2$ Abs. 1 Nr. 5 UWG.

282 Vgl. Kapitel 1A.

283 Vgl. hierzu nachfolgend Kapitel 3C

${ }^{284}$ Vgl. hierzu Kapitel 1A.

285 So bspw. in Art. 8 Abs. 2, Art. 10 Abs. 2 und Art. 16 E-Commerce-Richtlinie.

286 Micklitz in: MünchKomm, EG E, Rn. 198.

287 Maurer, $\ 24$, Rn. 1.
} 
haben Verwaltungsvorschriften nur innerhalb der Verwaltung eine unmittelbare Rechtswirkung, während sie im Außenbereich der Verwaltung lediglich mittelbar rechtlich wirken. ${ }^{288}$ Entsprechend kann sich aus ihnen nie eine direkte Verpflichtung von Privaten ergeben, so dass eine entsprechende Begriffsbedeutung in der UGP-Richtlinie ausscheidet. Nach dem Verständnis der Richtliniengeber sollten durch den Begriff der Verwaltungsvorschrift vielmehr sämtliche Rechtsakte erfasst werden, die eine mit Gesetzen vergleichbare Verbindlichkeit haben. ${ }^{289}$

Allerdings ist diese Voraussetzung insoweit einzuschränken, als auch deklaratorische Erklärungen, also Erklärungen, die lediglich zu rechtstreuem Verhalten verpflichten, indem sie sich auf die Wiederholung des Gesetzeswortlautes beziehungsweise die vereinfachte Wiedergabe des Regelungsinhaltes der gesetzlichen Regelung beschränken, ${ }^{290}$ als Verhaltenskodizes einzuordnen sind. Grund hierfür ist, dass entsprechende Erklärungen die Unternehmen auch außerhalb der Grenzen der Länder binden, nach deren Gesetzen oder Verwaltungsvorschriften sie in diesen zu dem jeweiligen Verhalten verpflichtet sind. ${ }^{291}$ Zudem spricht bereits die Häufigkeit entsprechender Verhaltenskodizes dafür, dass die Richtlinien- beziehungsweise der Gesetzgeber sie ausdrücklich ausgeschlossen hätte, wenn dies gewollt gewesen wäre. ${ }^{292}$

\section{B. Beschränkung auf Verpflichtungen von Unternehmern}

So unbestimmt die Definition im Hinblick auf den Inhalt von Verhaltenskodizes ist, so eindeutig bestimmt sie den persönlichen Anwendungsbereich (,Verhalten von Unternehmern, zu welchem diese ... sich verpflichtet haben ", Hervorhebung d. d. Verf.). Verhaltenskodizes i. S. d. UWG sind damit entgegen dem Begriffsverständnis im allgemeinen Sprachgebrauch ${ }^{293}$ nur Verhaltenskodizes von Unternehmern. Dies entspricht auch der Definition der UGP-Richtlinie, wobei diese nicht den Begriff des Unternehmers, sondern den des Gewerbetreibenden verwendet (, Verhalten der Gewerbetreibenden $\{\ldots\}$, die sich $\{. .$.$\} auf diesen Kodex verpflichten", Hervorhebung d. d.$ Verf.). Wer Unternehmer ist, ergibt sich dabei aus dem speziellen lauterkeitsrecht-

\footnotetext{
288 Maurer, $\ 24$, Rn. 16 ff. zur grundsätzlich allein bestehenden Innenwirkung, Rn. 20 ff. zur mittelbaren Außenwirkung.

289 Alexander, GRURInt 2012, 965, 969, der als Beispiele Allgemeinverfügungen und Verwaltungsakte benennt.

290 Dreyer, WRP 2007, 1294, 1297.

${ }^{291}$ So beispielsweise, wenn ein deutsches Unternehmen die in Deutschland gesetzlich vorgeschriebenen Urlaubsregelungen des Bundesurlaubsgesetzes für alle seine Angestellten weltweit für anwendbar erklärt.
}

292 Dreyer, WRP 2007, 1294, 1297.

${ }^{293}$ Vgl. Kapitel 1A. 
lichen Unternehmerbegriff des $₫ 2$ Abs. 1 Nr. 6 UWG. ${ }^{294}$ Dieser setzt die Definition des Gewerbetreibenden aus der UGP-Richtlinie ${ }^{295}$ inhaltlich ohne Abweichung um und ersetzt lediglich den Begriff des Gewerbetreibenden durch den des Unternehmers. ${ }^{296}$ Zur Begründung für diesen Begriffswechsel verweist der Gesetzgeber auf die Bedeutung des Begriffs des Gewerbetreibenden im deutschen Recht. Denn dieser umfasst im Gegensatz zu dem der UGP-Richtlinie keine handwerklichen und (anderen) beruflichen Tätigkeiten. ${ }^{297}$ Diese zu Recht vermiedene uneinheitliche Begriffsbedeutung hielt den Gesetzgeber jedoch nicht davon ab, als Ersatz den Begriff des Unternehmers auszuwählen und diesen abweichend von seiner Definition in $\ 12 \mathrm{BGB}^{298}$ für das UWG individuell zu definieren. So umfasst der Unternehmerbegriff des UWG im Gegensatz zu dem des BGB auch Personen, ,die im Namen oder Auftrag " eines Unternehmers handeln. Damit bestimmt das UWG (Hilfs)Personen, wie z. B. Mitarbeiter, formal zu Unternehmern, die gerade nicht unternehmerisch tätig sind. Andererseits werden der Rechtsfähigkeit nur angenäherte Personengesellschaften ${ }^{299}$ vom Wortlaut des lauterkeitsrechtlichen Unternehmerbegriffs nicht umfasst. ${ }^{300}$ Letzteres dürfte jedoch insofern ohne praktische Auswirkungen bleiben, als der Unternehmerbegriff des UWG richtlinienkonform auszulegen ist und entsprechende Personengesellschaften unter den Begriff der juristischen Person i. S. d. UGP-Richtlinie zu fassen sind. 301

Diese Beschränkung auf Verhaltenskodizes von Unternehmern bedeutet im Umkehrschluss, dass Verhaltenskodizes, die nicht von Unternehmern, sondern beispielsweise von Gewerkschaften, Nichtregierungsorganisationen, berufsständische Organisationen, Universitäten, Regierungen, Parlamenten und internationa-

\footnotetext{
294 \2 Abs. 1 Nr. 6 UWG: „,„Unternehmer“ jede natürliche oder juristische Person, die geschäftliche Handlungen im Rahmen ibrer gewerblichen, bandwerklichen oder beruflichen Tätigkeit vornimmt, und jede Person, die im Namen oder Auftrag einer solchen Person handelt;"

295 Art. 2 lit. b) UGP- Richtlinie: ,„,Gewerbetreibender" jede natürliche oder juristische Person, die im Geschäftsverkehr im Sinne dieser Richtlinie im Rahmen ibrer gewerblichen, handwerklichen oder beruflichen Tätigkeit handelt, und jede Person, die im Namen oder Auftrag des Gewerbetreibenden handelt;"“

296 Begründung der Bundesregierung für den Gesetzentwurf zur Änderung des UWG vom 20.08.2008, BT.-Drs. 16/10145, S. 21.

297 Vgl. Fn. 296.

298 S 14 BGB definiert den Unternehmer als ,eine natürliche oder juristische Person oder eine rechtsfähige Personengesellschaft, die bei Abschluss eines Recbtsgeschäfts in Ausübung ibrer gewerblichen oder selbständigen beruflichen Tätigkeit handelt. "Dieser Unternehmerbegriff gilt nicht nur im BGB, sondern wird in der gesamten deutschen (Zivil)Rechtsordnung verwendet. So verweist unter anderem $\$ 6$ PreisKIG auf $\ 14$ BGB. Auch das UWG 2004 verwies für die Definition des Unternehmers auf $\ 14$ BGB.

299 Wie die OHG oder die KG, IS 105, 124, 161 HGB.

${ }^{300}$ Entgegen $\int 14$ BGB, der hierfür in seinem Abs. 2 extra eine Sonderdefinition der rechtsfähigen Personengesellschaft aufstellt.

301 Sosnitza, WRP 2008, 1014, 1015.
} 
len Organisationen stammen, per definitionem außer Betracht bleiben. ${ }^{302}$ Ebenfalls nicht umfasst sind berufsständische Verhaltenskodizes. Dies ergibt sich aus einem Rückgriff auf die UGP-Richtlinie, welche diese laut ihrem Art. 3 Abs. 8 ausdrücklich unberührt lässt. Die Beschränkung auf Verhaltenskodizes, durch welche Unternehmer verpflichtet werden, bedeutet jedoch nicht, dass Verhaltenskodex auch von diesen erstellt werden müssen. Dies ergibt sich aus Definition des Urhebers eines Kodex in der UGP-Richtlinie ${ }^{303}$, die auch herangezogen werden kann und muss, obwohl der deutsche Gesetzgeber den Begriff selbst nicht in das UWG übernommen hat. ${ }^{304}$ Nach dieser können Verhaltenskodizes von jeder Rechtspersönlichkeit geschaffen werden, so dass neben den von Unternehmern und Unternehmensverbänden geschaffenen Verhaltenskodizes auch von natürlichen Personen, Umwelt- und Verbraucherschutzorganisationen und öffentlichen Stellen erstellte Regelwerke Verhaltenskodizes i. S. d. UWG sein können. ${ }^{305}$ Insbesondere die Beteiligung von Verbraucherverbänden an der Ausarbeitung von Verhaltenskodizes haben die Richtliniengeber ausdrücklich als sinnvoll erachtet, um das mit der UGP-Richtlinie verfolgte Ziel eines hohen Verbraucherschutzniveaus zu erreichen. ${ }^{306}$ Eine entsprechende Pflicht, diese zu beteiligen oder auch nur zu informieren, besteht jedoch nicht. ${ }^{307}$

Hinsichtlich der Anzahl der durch den Verhaltenskodex verpflichteten Unternehmer ist es notwendig, aber auch ausreichend, dass sich ein Unternehmer durch den Verhaltenskodex verpflichtet. ${ }^{308}$ Gegenteiliges ergibt sich auch nicht daraus, dass sowohl die Definition im UWG als auch die Definition in der UGPRichtlinie jeweils den Plural („von Unternehmern“ beziehungsweise „der Gewerbetreibenden") verwenden. ${ }^{309}$ Denn eine solche Einschränkung widerspricht zunächst einmal dem Willen der Richtliniengeber, da durch sie auch Verhaltenskodizes zwischen einem Unternehmen und einer NRO oder einer Gewerkschaft ausgeschlossen würden, welche in jedem Fall umfasst sein sollten. Zudem steht dieser einschränkenden Auslegung entgegen, dass die gesamte Definition im Plural abgefasst ist, jedoch in keinem Fall vorausgesetzt werden kann, dass gleich mehrere Vereinbarungen oder Vorschriften getroffen werden. Insofern ist der Wortlaut diesbezüglich — trotz der Verwendung des Plurals — nicht eindeutig, so dass eine solche einschränkende Lesart der Definition ausscheidet.

\footnotetext{
302 Ohnehin wäre das UWG auf diese Verhaltenskodizes nur in den seltensten Fällen anwendbar, da diesen der Marktbezug fehlt, so dass bereits keine geschäftliche Handlung vorliegt, vgl. hierzu nachfolgend Kapitel 4A.

303 Art. 2 lit. g) UGP-Richtlinie, vgl. zum Inhalt Fn. 260.

$304 \mathrm{Vgl}$. Kapitel 2B.II.

305 Büllesbach, S. 22.

306 Erwägungsgrund 20 UGP-Richtlinie.

307 Alexander, GRURInt 2012, 965, 967.

308 Alexander, GRURInt 2012, 965, 967.

309 A. A. Birk, GRUR 2011, 196, 199.
} 


\section{Gegenstand der Verpflichtung}

Im Hinblick auf den Gegenstand der mit einem Verhaltenskodexes eingegangenen Verpflichtung beschränkt sich die Definition aus $\int 2$ Abs. 1 Nr. 5 UWG auf den Oberbegriff des Verhaltens. Somit reicht nach dem Wortlaut der Definition eine Verpflichtung zu jeglichem Tun oder Unterlassen eines Unternehmens aus. ${ }^{310}$ Unter Hinweis auf den Sinn und Zweck der UGP-Richtlinie wird jedoch vielfach als ungeschriebene Voraussetzung für die Einordnung als Verhaltenskodex i. S. d. UWG gefordert, dass die Verpflichtung das lautere Verhalten des Unternehmens im Wettbewerb betreffen müsse. ${ }^{311}$ Als Begründung hierfür wird auf Erwägungsgrund 20 der UGP-Richtlinie verwiesen. ${ }^{312}$ Aus diesem ergibt sich, dass der Richtliniengeber es als zweckmäßig erachtet, ,die Möglichkeit von Verhaltenskodizes vorzusehen, die es Gewerbetreibenden ermöglichen, die Grundsätze dieser Richtlinie in spezifischen Wirtschaftsbranchen wirksam anzuwenden."313 In besonderer Vehemenz erkennt Büllesbach314 sogar nur solche Verhaltenskodizes als Verhaltenskodizes i. S. d. UGPRichtlinie an, die mit dem Ziel aufgestellt werden, ,die Gebote des lauteren Wettbewerbs zu konkretisieren“. Verhaltenskodizes auf anderen, nicht unmittelbar lauterkeitsbezogen Gebieten sollen dahingegen nicht dem Begriff des Verhaltenskodexes i. S. d. UGP-Richtlinie unterfallen. ${ }^{315}$ Als Beispiele hierfür nennt Büllesbach unter anderem Verhaltenskodizes über die Einhaltung bestimmter Umweltstandards in der Produktion. 316

Eine solche Begrenzung auf Verhaltenskodizes mit lauterkeitsrechtlichem Bezug mit dem damit verbundenen Ausschluss von Verhaltenskodizes aus dem Bereich des Umweltschutzes oder der sozialen Mindeststandards steht jedoch im Widerspruch zum Begriffsverständnis der Europäischen Kommission. Deren Begriffsverständnis ergibt sich aus dem von der Generaldirektion Gesundheit und Verbraucher ${ }^{317}$ erstellten Arbeitspapier „Leitlinien zur Umsetzung/Anwendung der Richtlinie 2005/29/EG über unlautere Geschäftspraktiken".318 Dieses 2009 — und damit nach der Dissertation von Büllesbach — veröffentlichte Arbeitspapier beruht „auf den Ergebnissen der Zusammenarbeit der Kommission mit den Mitgliedstaaten und den

\footnotetext{
310 Hoeren, WRP 2009, 789, 793, Dreyer, WRP 2007, 1294, 1295 ff., Schmidbauer, WRP 2010, 593, 597.

311 Dreyer, WRP 2007, 1294, 1296, Beater, \ 16, Rn. 1273 und Köhler in: Köhler/Bornkamm, UWG, \2, Rn. 113, a. A. Lamberti/Wendel, WRP 2009, 1479, 1482 f., denen ein lauterkeitsrechtlichen Bezug nicht zwingend erscheint.

312 Dreyer, WRP 2007, 1294, 1296, Beater, $\mathbb{1 6}$, Rn. 1273 und Köhler in: Köhler/Bornkamm, UWG, $\$ 2$, Rn. 113.

313 Erwägungsgrund 20 der UGP-Richtlinie.

314 Büllesbach, S. 43.

315 Vgl. Fn. 314.

316 Vgl. Fn. 314.

317 Auf welche die UGP-Richtlinie zurückgeht, vgl. Kapitel 2B.I.1.a.

318 Leitlinien zur Umsetzung der UGP-Richtlinie, SEK(2009)1666.
} 
betroffenen Parteien in den Jahren nach der Annahme der Richtlinie“. Es sollte dazu dienen, „Leitlinien zu den wichtigsten als problematisch eingeschätzten Konzepten und Bestimmungen (der UGP-Richtlinie, Ergänzung d. d. Verf.) bereitzustellen“. Hierzu geht das Arbeitspapier auf zahlreiche Fragen von einzelstaatlichen Behörden und Betroffenen ein und bedient es sich dabei praktischer Beispiele. ${ }^{319}$ Eines dieser Beispiele betrifft Verstöße gegen Verhaltenskodizes mit Umweltschutzverpflichtungen und sieht in diesen Verstößen einen möglichen Fall der Irreführung nach Art. 6 Abs. 2 lit. b) UGP-Richtlinie. ${ }^{320}$ Dabei lautet das Beispiel wie folgt:

„Ein Gewerbetreibender hat einen verbindlichen Verhaltenskodex unterzeichnet, der eine $V$ erpflichtung zur nachbaltigen Nutzung von Holz enthält, und verwendet das Logo des Verhaltenskodex auf seiner Website. Der Verhaltenskodex besagt, dass die Unterzeichner kein Hartholz aus nicht nachbaltig bewirtschafteten Wäldern verwenden dïrfen. Es wird jedoch festgestellt, dass die auf der Website beworbenen Produkte Holz aus Rodungsgebieten enthalten"

Da dieser Verhaltenskodex allein die Verpflichtung nur nachhaltigen Holznutzung enthält und keinerlei lauterkeitsrechtlichen Bezug hat, ist nicht nur die strenge Ansicht von Büllesbach, sondern jegliches Erfordernis eines lauterkeitsrechtlichen Bezuges als ungeschriebenes Tatbestandsmerkmal eines Verhaltenskodexes i. S. d. UGP-Richtlinie — und damit auch i. S. d. UWG — abzulehnen. Dies gilt trotz des fehlenden formalrechtlichen Status des Arbeitspapiers ${ }^{321}$ und der Tatsache, dass in letzter Instanz selbstverständlich der EuGH und nicht die Europäische Kommission für die Auslegung der UGP-Richtlinie zuständig ist.

Unabhängig von der Ablehnung eines lauterkeitsrechtlichen Bezuges als ungeschriebenes Tatbestandsmerkmal reicht es anderseits jedoch nicht aus, lediglich auf den Wortlaut der Definition aus $\int 2$ Abs. 1 Nr. 5 UWG abzustellen und die Verpflichtung zu jeglichem Verhalten ausreichen zu lassen. ${ }^{322}$ Denn dies würde dazu führen, dass auch Regelwerke als Verhaltenskodizes i.S.d. \2 Abs. 1 Nr. 5 UWG einzuordnen wären, die weder nach dem allgemeinen Sprachgebrauch $^{323}$ noch nach dem Begriffsverständnis der Richtliniengeber mit einem Verhaltenskodex in Verbindung gebracht werden können. ${ }^{324}$ Letztere verstehen Verhaltenskodizes in weitestgehender Übereinstimmung mit dem allgemeinen

319 Leitlinien zur Umsetzung der UGP-Richtlinie, SEK(2009)1666, Einleitung, S. 7.

320 Leitlinien zur Umsetzung der UGP-Richtlinie, SEK(2009)1666, Punkt 2.5.6., S. 51 f.

321 Die Leitlinien zur Umsetzung der UGP-Richtlinie (SEK(2009)1666) sind ausweislich ihres „Haftungsausschlusses" auf S. 2 ausdrücklich, f̈̈̈r die Europäische Kommission als Organ nicht bindend" und bieten ,weder eine formelle situationsberogene Auslegung des Gemeinschaftsrechts noch Rechtsberatung zu Fragen einzelstaatlicher Rechtsvorschriften".

322 Hoeren, WRP 2009, 789, 793, Dreyer, WRP 2007, 1294, 1295 ff., Schmidbauer, WRP 2010, 593, 597.

323 Vgl. hierzu Kapitel 1A.

324 Schmidbauer, WRP 2010, S. 593, 598. 
Sprachgebrauch ${ }^{325}$ als ,formelle Erklärungen $z$ den Werten und Aktivitäten eines Unternehmens"326 sowie als „Instrumente zur Förderung grundlegender Menschen-, Arbeits- und Umweltrechte sowie der Korruptionsbekämpfung, insbesondere in Ländern obne diesberügliche Mindeststandards". 327

So wird unter Bezugnahme auf den Wortlaut der Definition eines Verhaltenskodexes aus $\int 5$ Abs. 1 Nr. 5 UWG beispielsweise vertreten, Vertriebsbindungssysteme als Verhaltenskodizes i. S.d. UWG einzuordnen. ${ }^{328}$ Als Vertriebsbindungssysteme werden Regelwerke bezeichnet, durch welche ein Hersteller oder ein von ihm bestimmter Generalimporteur bestimmte Händler auswählt, die seine Waren exklusiv vertreiben dürfen. Dabei können diese Vertriebsbindungssysteme sowohl eine qualitative, als auch eine quantitative Auswahl vornehmen. ${ }^{329}$ Ziel dieser Vertriebsbindungssysteme ist es, dass die Waren des Herstellers nur in einem geschlossenen Händlernetz verfügbar sind. ${ }^{330}$ Aus diesem Grund ist die Verpflichtung der ausgewählten Händler, nicht an systemfremde Wiederverkäufer zu verkaufen, zentrales Element solcher Regelwerke. ${ }^{331}$ Obwohl selbst die Befürworter einer Einordnung von Vertriebsbindungssystemen als Verhaltenskodizes nicht verhehlen, dass diese etwas anderes sind als die Verhaltenskodizes, an welchen sich die UGP-Richtlinie orientiert ${ }^{332}$, erfüllen Vertriebsbindungssysteme sämtliche Voraussetzungen der Definition aus $₫ 5$ Abs. 1 Nr. 5 UWG, soweit nur auf den Wortlaut abgestellt wird. Denn es handelt sich bei ihnen um Vereinbarungen, mit welchen sich Unternehmer auf ein bestimmtes Verhalten verpflichten, ohne dass eine entsprechende Verpflichtung aus Gesetzes- oder Verwaltungsvorschriften besteht. Aber nicht nur Vertriebsbindungssysteme erfüllen die Voraussetzungen der Definition aus $₫ 5$ Abs. 1 Nr. 5 UWG. Vielmehr dürfte dies mit wenigen Ausnahmen für alle Verträge gelten, durch welche sich ein Unternehmer verpflichtet. ${ }^{333}$ Wäre dies der Fall, wären Verstöße gegen entsprechende Verträge gleichfalls Verstöße gegen Verhaltenskodizes, was wiederum Rechtsfolgen aufgrund des Lauterkeitsrechts nach sich ziehen könnte. Dies würde jedoch in fundamentaler Art und Weise dem Willen der Richtliniengeber widersprechen. Denn ausweislich Art. 3 Abs. 2 UGP-Richtlinie, welcher den Anwendungsbereich der UGP-

\footnotetext{
325 Vgl. Kapitel 1A.

326 Grünbuch CSR, Anhang Begriffsbestimmungen, S. 28.

327 Mitteilung der Kommission über die im Nachgang zum Grünbuch CSR erfolgten Konsultationen mit Arbeitgeberorganisationen, Gewerkschaften und Einzelpersonen, KOM (2002) 347 endgültig, S. 15.

${ }^{328}$ Hoeren, WRP 2009, 789, 793 f. sowie insbesondere Lamberti/Wendel, WRP 2009, 1479, 1482 ff.

${ }^{329}$ Rosner, S. 1 f.

330 Rosner, S. 3.

331 Rosner, S. 3, Schmidhuber, WRP 2010, 593, 596.

332 Lamberti/Wendel, WRP 2009, 1479, 1481.

333 Schmidbauer, WRP 2010, S. 593, 597.
} 
Richtlinie bestimmt, lässt diese „das Vertragsrecht und insbesondere die Bestimmungen über die Wirksamkeit, das Zustandekommen oder die Wirk.ungen eines Vertrags unberührt."

Die dementsprechend vorzunehmende Eingrenzung muss direkt über eine einschränkende Auslegung des Begriffs Verbalten erfolgen. Denn weder dem positiv (,Vereinbarungen oder Vorschriften über das Verhalten von Unternehmern, zu welchem diese sich im Bezug auf Wirtschaftszweige oder einzelne geschäftliche Handlungen verpflichtet haben "), noch dem negativ (,ohne dass sich solche Verpflichtungen aus Gesetzes- oder Verwaltungsvorschriften ergeben. ") formulierten Teil der Definition kann eine Eingrenzung der erfassten Verhaltensweisen entnommen werden. Insbesondere trägt die Vorgabe, dass diese Verpflichtung als Vereinbarung und Vorschriften(katalog) ${ }^{334}$ zu erfolgen hat, nicht zur inhaltlichen Eingrenzung bei. Denn eine Vereinbarung beziehungsweise ein Vorschriftenkatalog ist nichts anderes als die formale Hülle um eine Verpflichtung (zu einem bestimmten Verhalten). So ist der Begriff des Verhaltens in der Definition des $\int 5$ Abs. 1 Nr. 5 UWG nicht als ein beliebiges, konkret zu beschreibendes Verhalten zu verstehen ${ }^{335}$, sondern im Sinne eines grundsätzlichen und auf Dauer angelegten verantwortungsbewussten Verhaltens. Voraussetzung für die Einordnung einer Vereinbarung oder Vorschrift als Verhaltenskodizes i. S. d. UWG ist daher, dass diese nach dem Begriffsverständnis der Richtliniengeber und des allgemeinen Sprachgebrauchs als Verhaltenskodex anzusehen ist. Dies dürfte immer dann nicht der Fall sein, wenn die Vereinbarung zwischen zwei wirtschaftlich verbundenen Parteien geschlossen wird und wirtschaftlich äquivalente Ansprüche für die Beteiligten enthält.

\section{Notwendiger Grad der Verpflichtung}

Weitere Voraussetzung für das Vorliegen eines Verhaltenskodexes ist die Verpflichtung des jeweiligen Unternehmers auf dieses Verhalten. Diesbezüglich ist umstritten, welchen Grad der Verpflichtung die Definition voraussetzt. ${ }^{336}$ Aufhänger der Auseinandersetzung sind dabei die sogenannten Absichtserklärungen. Hierunter sind nicht die im Bereich der Wirtschaft verwendeten „Letters of Intent zu verstehen, mit welchen sich die Parteien ohne rechtliche Verpflichtungen gegenseitig versichern in Vertragsverhandlungen zu stehen. Gemeint sind Regelwerke, die keine Verpflichtung auf ein Verhalten vorsehen, sondern nur die Absicht oder das Bemühen bekunden, sich in einer bestimmten Art und Weise zu verhalten. Aus den Definitionen im UWG sowie in der UGP-Richtlinie lässt sich diesbezüglich — wenn überhaupt — nur aus den Begrifflichkeiten Vereinbarung und

\footnotetext{
334 So die Begrifflichkeit in der UGP-Richtlinie, welche bei der Auslegung zugrunde zulegen ist.

335 Wie beispielsweise die Nichtweitergabe von Waren an Wiederverkäufer, wie sie in Vertriebsbindungssystemen vorgeschrieben wird.

${ }^{336}$ Für eine enge Auslegung aufgrund des Wortlautes in der Definition (,sich $\{\ldots\}$ verpflichten“), Büllesbach, S. 43, für eine weite Auslegung Dreyer, WRP 1294, 1296 f.
} 
Vorschriften(katalog) eine besondere Verbindlichkeit ableiten. ${ }^{337}$ Dass tatsächlich die weitere Auslegung geboten ist, ergibt sich jedoch aus $₫ 5$ Abs. 1 Nr. 6 UWG unter Rückgriff auf Art. 6 Abs. 2 lit. b) UGP-Richtlinie. Denn während $\ 5$ Abs. 1 Nr. 6 UWG sich nur auf Angaben über die Einhaltung eines Verhaltenskodexes beschränkt, auf den sich der Unternehmer ,verbindlich verpflichtet hat", nimmt seine Ursprungsregelung in der UGP-Richtlinie explizit Bezug auf Absichtserklärungen, indem sie (nur solche) Geschäftspraktiken als irreführend bestimmt ${ }^{338}$, welche „die Nicbteinhaltung von Verpflichtungen, die der Gewerbetreibende im Rabmen von Verbaltenskodizes, auf die er sich verpflichtet hat, eingegangen ist, sofern i) es sich nicht um eine Absichtserklärung, sondern um eine eindeutige Verpflichtung handelt (Hervorhebung d. d. Verf.) (...)“ beinhalten. Diese Regelung ergibt jedoch nur dann einen Sinn, wenn sie eine Konkretisierung der generellen Anforderungen an Verhaltenskodizes im Allgemeinen für die Fälle des Art. 6 Abs. 2 lit. b) UGP-Richtlinie beinhaltet. Auf dieser Grundlage ergibt sich aus der Tatsache, dass der Richtliniengeber Absichtserklärungen ausdrücklich aus dem Anwendungsbereich von Art. 6 Abs. 2 lit. b) UGPRichtlinie herausnimmt, dass Absichtserklärungen grundsätzlich Verhaltenskodizes i. S. d. UGP-Richtlinie sein können. Dies gilt im Zuge der richtlinienkonformen Auslegung somit auch für Verhaltenskodizes i. S. d. UWG.

\section{E. Notwendiger Inhalt von Verhaltenskodizes}

Teilweise wird in der Literatur vertreten, dass eine Vereinbarung oder Vorschrift nur dann einen Verhaltenskodex i. S. d. UWG darstellen kann, wenn sie Maßnahmen für die Überwachung der mit ihr eingegangenen Verpflichtungen enthält. ${ }^{339}$ Diese aus der Definition des Urhebers eines Kodexes ${ }^{340}$, welchen der deutsche Gesetzgeber nicht in das UWG übernommen hat ${ }^{341}$, entnommene Voraussetzung ist jedoch abzulehnen. Denn es besteht keine berechtigte Veranlassung, die Anforderungen an einen Verhaltenskodex um eine solche Voraussetzung zu erhöhen, die eine Vielzahl der bestehenden Verhaltenskodizes aus dem Anwendungsbereich

\footnotetext{
337 So auch Dreyer, WRP 2007, 1294, 1297 bzgl. der verpflichtenden Natur der Begrifflichkeiten, jedoch konträr bzgl. des Ergebnisses.

338 Wenn sie kumulativ einen Verbraucher zu einer geschäftlichen Handlung veranlasst oder zu veranlassen geeignet ist, die er ansonsten nicht getroffen hätte, Art. 6 Abs. 2 UGP-Richtlinie.

339 Keßler/Micklitz, Special zu Betriebs Berater 2005, Beilage 13, S. 1, 20.

340 Art. 2 lit. g) UGP-Richtlinie: „Urheber eines Kodex“ jede Rechtspersönlichkeit, einschließlich einzelner Gewerbetreibender oder Gruppen von Gewerbetreibenden, die für die Formulierung und Überarbeitung eines Verhaltenskodex und/ oder für die Überwachung der Einhaltung dieses Kodex durch alle diejenigen, die sich darauf verpflichtet baben, zuständig ist;" "

${ }^{341}$ Der Begriff und entsprechend auch seine Definition wurden nicht in das UWG übernommen, da der Gesetzgeber keine Verwendung für ihn sah und entsprechend auch keinen Umsetzungsbedarf, Begründung der Bundesregierung für den Gesetzentwurf zur Änderung des UWG vom 20.08.2008, BT.-Drs. 16/10145, S. 12.
} 
des UWG ausschließen würde. ${ }^{342}$ Zudem könnte eine solche Voraussetzung böswillig ausgenutzt werden, indem sich Unternehmen einem Verhaltenskodex ohne entsprechende Regelungen abgeben und bei Inanspruchnahme der Ansprüche des Lauterkeitsrechts auf die fehlende Qualität als Verhaltenskodex i. S. d. UWG verweisen würden.

\section{F. Kartellrechtliche Problematik von Verhaltenskodizes i. S. d. $\$ 2$ Abs. 1 Nr. 5 UWG}

Auch wenn diese Arbeit die rechtliche Behandlung von Verhaltenskodizes im Lauterkeitsrecht betrifft, soll an dieser Stelle in der gebotenen Kürze auf deren kartellrechtliche Zulässigkeit eingegangen werden. Grund hierfür ist nicht allein das besondere Verhältnis zwischen den sich ergänzenden Rechtsbereichen Lauterkeits- und Kartellrecht, die zusammen das Wettbewerbsrecht bilden, sondern vielmehr die augenscheinlich Nähe von Verhaltenskodizes, an denen mindestens zwei Unternehmen beteiligt sind ${ }^{343}$, zu Kartellvereinbarungen.

Denn gemäß \1 GWB (beziehungsweise inhaltlich deckungsgleich gemäß Art. 101 Abs. 1 AEUV ${ }^{344}$ ) sind Vereinbarungen zwischen Unternehmen über "Verhaltensweisen, die eine Verhinderung, Einschränkung oder Verfälschung des Wettbewerbs bezwecken oder bewirken" verboten. Da das Tatbestandsmerkmal der Einschränkung des Wettbewerbs jede Beschränkung der wettbewerblichen Handlungsfreiheit umfasst, reicht zu seiner Erfüllung die Verpflichtung aus, auf bestimmte Formen der Werbung oder bestimmte Produktionsbedingungen $\mathrm{zu}$ verzichten ${ }^{345}$, die in Verhaltenskodizes i. S. d. UWG zum Standard gehören. Soweit der Verhaltenskodex von einer großen Zahl von Wettbewerbern (beispielsweise von allen Mitgliedern eines Branchenverbandes) ${ }^{346}$ unterzeichnet wurde, wird es den Verhaltenskodex zudem kaum an dem ungeschriebenen Tatbestandsmerkmal der Spürbarkeit fehlen. ${ }^{347}$ Insofern erscheint es mehr als nur möglich, dass $₫ 1$ GWB beziehungsweise Art. 101 Abs. 1 AEUV einschlägig sein können. ${ }^{348}$

\footnotetext{
342 Vgl. hierzu die Beispiele unter Kapitel 1C.

${ }^{343}$ Dabei reicht es für die Annahme der Beteiligung von 2 Unternehmen nicht aus, dass ein Verhaltenskodex, wie in dem unter Kapitel 1C.I.2 dargestellten Beispiel der Otto-Gruppe, einen gesamten Konzern und damit viele, im rechtlichen Sinn selbstständige Unternehmen betrifft. Denn solche Konzerne werden kartellrechtlich als ein Unternehmen behandelt, Bechtold in: Bechtold, Kommentar zum GWB, \& 1, Rn. 23 ff.

344 Art. 101 Abs. 1 AEUV ist anwendbar, wenn der Handel zwischen Mitgliedstaaten betroffen ist.

345 Bechtold in: Bechtold, Kommentar zum GWB, \1, Rn. 28.

346 So beispielsweise das Responsible Care Programm der Chemieindustrie, vgl. Kapitel 1C.II.2

347 Bornkamm in: Festschrift für Griss, S. 90.

348 Sosnitza in: FS für Bechtold, S. 517.
} 
Aus diesem Grund ist Bornkamm zuzustimmen, wenn er es als erstaunlich bezeichnet, dass die UGP-Richtlinie, trotz dieser definitionsbedingten Nähe von Verhaltenskodizes gemäß ihres Art. 2 lit. f) zur Verbotszone des Art. 101 AEUV, mit keinem Wort auf diese Problematik eingeht. ${ }^{349}$

\section{Verhaltenskodizes als unzulässige Kartellvereinbarung}

Unabhängig von der Einschlägigkeit der Tatbestände aus $\int 1$ GWB beziehungsweise Art. 101 Abs. 1 AEUV kommt eine kartellrechtliche Unzulässigkeit unstreitig nicht in Betracht, sofern sich ein Verhaltenskodex inhaltlich auf das Verbot von Verhaltensweisen beschränkt, die nach dem UWG unzulässig sind. Grund hierfür ist, dass das GWB nur den lauteren Wettbewerb schützt, so dass Vereinbarungen über das Verbot des unlauteren Wettbewerbs keine unzulässigen Kartellvereinbarungen gem. $\int 1$ GWB beziehungsweise Art. 101 Abs. 1 AEUV sind. ${ }^{350}$

Umstritten ist dahingegen die kartellrechtliche Zulässigkeit von Verhaltenskodizes, die nach dem UWG zulässige Verhaltensweisen unmittelbar verbieten beziehungsweise, in Umsetzung des Willens der Richtliniengeber ${ }^{351}$, Anforderungen an die fachliche Sorgfalt in ihrer Branche definieren und Verstöße gegen diese fachliche Sorgfalt verbieten. 352

Juristischer Ausgangspunkt dieser Streitfrage ist der Begriff des leistungsgerechten Wettbewerbs, der in der Definition der Wettbewerbsregel aus $\$ Abs. 24 Abs. 2 GWB $^{353}$ gleichberechtigt neben dem Begriff des lauteren Wettbewerbs steht. Diesem entnimmt ein Teil der Literatur, dass nicht nur Regelungen, die sich auf die Unlauterkeitstatbestände des UWG beschränken, keine unzulässigen Kartellvereinbarungen sind, sondern auch solche, die geschäftliche Handlungen untersagen, die sich im Vorfeld der Unlauterkeit befinden beziehungsweise eine Missbrauchstendenz aufweisen. Die Gegenmeinung in der Literatur kritisiert diese Erweiterung des kartellrechtlichen Zulässigkeitsbereiches aufgrund dieses (zu) unbestimmten Rechtsbegriffs und gesteht Wettbewerbsregeln über das Verbot von nach dem UWG unzulässigen geschäftlichen Handlungen hinaus maximal zu, solche Verhaltensweisen zu verbieten, denen eine eindeutige Tendenz zur Unlauterkeit innewohnt. 354

\footnotetext{
${ }^{349}$ Bornkamm in: Festschrift für Griss, S. 89, der entsprechende Verpflichtungen auf ein bestimmtes Verhaltens bzw. dessen Unterlassen an dieser Stelle als "klassisches Kartell" bezeichnet.

350 Urteil des BGH v. 26.10.1961 - KZR $3 / 61$ in NJW 1962, 247, 248, IV, b), Sosnitza in: FS für Bechtold, S. 517

351 Erwägungsgrund 20 der UGP-Richtlinie.

352 Bornkamm in: Festschrift für Griss, S. 90.

${ }^{353}$ Nach $\ 24$ Abs. 2 GWB sind Wettbewerbsregeln „Bestimmungen, die das Verhalten von Unternehmen im Wettbewerb regeln zu dem Zweck, einem den Grundsätzen des lauteren oder der Wirksamkeit eines leistungsgerechten Wettbewerbs zuwiderlaufenden Verbalten im Wettbewerb entgegenzwowirken und ein diesen Grundsätzen entsprechendes Verbalten im Wettbewerb anzuregen."

354 Sosnitza in: FS für Bechtold, S. 518.
} 
Nach Ansicht des Verfassers ist die weitere Auslegung vorzugswürdig, da andernfalls die gesetzliche Regelung und damit der Wille des Gesetzgebers unzulässig beschnitten werden würde. Denn ohne Zweifel wollte der Gesetzgeber den Bereich der kartellrechtlich zulässigen Wettbewerbsregeln durch die Aufnahme des Begriffs des leistungsgerechten Wettbewerbs erweitern. ${ }^{355}$ Zudem würde eine Begrenzung des kartellrechtlich zulässigen Inhalts auf durch das UWG verbotene geschäftliche Handlungen bei Verhaltenskodizes, welche, wie von den Richtliniengebern ausdrücklich gewünscht ${ }^{356}$, Anforderungen an die fachliche Sorgfalt enthalten und deren Nichteinhaltung verbieten, zu mit dem allgemeinen Rechtsempfinden nicht zu vereinbarenden Ergebnissen führen.

In diesen Fällen wäre unter Zugrundelegung der engeren Auslegung für die Entscheidung über die kartellrechtliche Zulässigkeit nämlich ausschlaggebend, ob sich die Anforderungen an die fachliche Sorgfalt in dem Verhaltenskodex mit den Anforderungen decken, welche die Rechtsprechung an die fachliche Sorgfalt in der jeweiligen Branche stellt. Decken sich die Anforderungen an die fachliche Sorgfalt, wäre der Verhaltenskodex kartellrechtlich zulässig, da er lediglich Verhaltensweisen untersagt, die auch nach dem UWG verboten sind. ${ }^{357}$ Decken sich die Anforderungen an die fachliche Sorgfalt jedoch nicht, wären die in dem Verhaltenskodex verbotenen Verhaltensweisen lauterkeitsrechtlich zulässig und der Verhaltenskodex damit kartellrechtlich unzulässig.

Dies ist mit dem allgemeinen Rechtsempfinden nicht zu vereinbaren, da die UGP-Richtlinie selbst in Erwägungsgrund 20 die eindeutige Aufforderung enthält, die nach Ansicht der erstellenden Branchenvereinigungen geltenden Anforderungen an die fachliche Sorgfalt in ihrer Branche in ihre Verhaltenskodizes aufzunehmen. ${ }^{358}$ Es kann aber nicht angehen, dass die Beteiligten an einem solchen Verhaltenskodex aufgrund der Tatsache, dass sie dem Willen der Richtliniengeber entsprechen, der Gefahr von Maßnahmen nach dem Kartellrecht ausgesetzt werden. Zumindest Verhaltenskodizes, die Anforderungen an die berufliche Sorgfalt definieren, dürfen aus diesem Grund keine unzulässigen Kartellvereinbarungen darstellen.

${ }^{355}$ Der Begriff des leistungsgerechten Wettbewerbs wurde durch die 2. GWB-Novelle im Jahr 1973 eingefügt.

356 Erwägungsgrund 20 der UGP-Richtlinie. Dieser verwendet zwar den Begriff der beruflichen Sorgfalt, dieser ist jedoch mit dem im UWG verwendeten Begriff der fachlichen Sorgfalt inhaltlich identisch, Regierungsentwurf und Begründung zum UWG vom 20.08.2008, BT.-Drs. 16/10145, S. 22 f., vgl. hierzu Kapitel 6D.I).

357 Zur Unzulässigkeit von Verstöße gegen die fachliche Sorgfalt vgl. Kapitel 6D.

358 Zur beschränkten Bedeutung der Angaben zur fachlichen Sorgfalt in Verhaltenskodizes zur Ermittlung der fachlichen Sorgfalt durch die Justiz vgl. Kapitel 6D.II.2.c. 
II. Freistellung gemäß $₫ 2$ GWB beziehungsweise Art. 101 Abs. 3 AEUV

Unstreitig von keiner der vorgenannten Ansichten umfasst sind dahingegen Verhaltenskodizes mit ökologischen, ethischen oder sozialen Verpflichtungen, so dass diese zunächst einmal $\ 1$ GWB beziehungsweise Art. 101 Abs. 1 AEUV unterfallen. Dies ist jedoch im Hinblick auf die kartellrechtliche Zulässigkeit solcher Verhaltenskodizes irrelevant, da diese die Anforderungen an eine Freistellung vom Verbot der unzulässigen Kartellvereinbarungen gemäß $\ 2$ Abs. 1 GWB beziehungsweise Art. 101 Abs. 3 AEUV erfüllen. ${ }^{359}$ Denn es ist aufgrund der nicht unbegrenzt bestehenden Ressourcen eine Förderung des wirtschaftlichen Fortschritts ${ }^{360}$, wenn im Rahmen der Produktion von Waren nur nachhaltig an- und abgebaute Rohstoffe verwendet werden und auf umweltschädliche Substanzen verzichtet wird. Gleiches gilt für eine angemessene Entlohnung sowie Maßnahmen zum Schutz der Gesundheit von Arbeitskräften. ${ }^{361}$ An dem Gewinn dieses wirtschaftlichen Fortschritts sind die Verbraucher auch angemessen beteiligt ${ }^{362}$, da sie bessere, weil nachhaltige( $r$ ) und ethisch korrekt(er) erzeugte Produkte angeboten bekommen. Eine Freistellung würde (nur) dann ausscheiden, wenn der jeweilige Verhaltenskodex zur Erreichung seiner Ziele unnötige Beschränkungen enthalten würde oder geeignet wäre, den Wettbewerb auszuschalten. ${ }^{363}$

Dahingegen ist eine Förderung des wirtschaftlichen Fortschritts bei Verhaltenskodizes, die Regelungen zur Lauterkeit bestimmter Verhaltensweisen enthalten, nicht in dieser Eindeutigkeit zu erkennen. Selbst wenn eine solche angenommen würde, ist eine Beteiligung der Verbraucher an dem dadurch entstehenden Gewinn nur schwer zu erkennen. Da für diese Verhaltenskodizes eine Freistellung gemäß \2 Abs. 1 GWB beziehungsweise Art. 101 Abs. 3 AEUV damit ausschei$\operatorname{det}^{364}$, wären diese unter Anwendung der engen Auslegung ${ }^{365}$ kartellrechtswidrig.

III. Anerkennung von Verhaltenskodizes als Wettbewerbsregel gem. \26 Abs. 1 Satz 1 GWB

Gemäß $\$ Abs. 26 Abs. 1 Satz 1 GWB können Wettbewerbsregeln, die keine unzulässige Kartellvereinbarung gemäß $\$ 1$ GWB darstellen, durch die Kartellbehörde

\footnotetext{
359 Bornkamm in: Festschrift für Griss, S. 90.

${ }^{360}$ Die Förderung des wirtschaftlichen Fortschritts ist eine der Tatbestandsvoraussetzungen der Freistellung gem. \& 2 Abs. 1 GWB.

361 Bornkamm in: Festschrift für Griss, S. 90.

362 Die angemessene Beteiligung der Verbraucher an dem erzielten Gewinn ist eine weitere Tatbestandsvoraussetzungen der Freistellung gem. \ 2 Abs. 1 GWB.

${ }^{363}$ Entsprechend der Rückausschlusstatbestände gemäß $\int 2$ Abs. 1 Nr. 1 und Nr. 2 GWB bzw. Art. 101 Abs. 3 lit. a) oder lit. b) AEUV.

${ }^{364}$ Gleiches gilt für die Freistellung gemäß $₫ 3$ GWB, da diese Verhaltenskodizes auch keine Rationalisierung wirtschaftlicher Vorgänge zum Gegenstand haben.

365 Vgl. Kapitel 3F.I.
} 
anerkannt werden. Diese Anerkennung hat zur Folge, dass die Kartellbehörde sich verpflichtet, von den ihr zustehenden Maßnahmen keinen Gebrauch zu machen. ${ }^{366}$ Allerdings bindet $\int$ Abs. 26 Abs. 1 Satz 2 GWB nur die Kartellbehörde, so dass in Drittprozessen die Kartellrechtswidrigkeit entsprechender Wettbewerbsregeln Verfahrensgegenstand sein kann. ${ }^{367}$

Da Verhaltenskodizes i. S. d. \ 2 Abs. 1 Nr. 5 UWG in aller Regel keine unzulässige Kartellvereinbarung gemäß \$1 GWB darstellen, können sie von den Privilegien der Anerkennung gemäß \ Abs. 26 Abs. 1 Satz 1 GWB profitieren, soweit sie der Definition der Wettbewerbsregel aus $\int$ Abs. 24 Abs. 2 GWB unterfallen. ${ }^{368}$ Nach Ansicht von Bornkamm besteht zwischen beiden kein wesentlicher Unterschied $^{369}$ beziehungsweise handelt es sich bei Wettbewerbsregeln gemäß \24 Abs. 2 GWB um Verhaltenskodizes gemäß $\int 2$ Abs. 1 Nr. 5 UWG. ${ }^{370}$ Letzterem ist in jedem Fall zuzustimmen, jedoch unter der Beachtung der Tatsache, dass im Gegenzug nicht jeder Verhaltenskodizes i. S. d. \ 2 Abs. 1 Nr. 5 UWG eine Wettbewerbsregel i. S. d. $\int$ Abs. 24 Abs. 2 GWB darstellt. ${ }^{371}$ Denn wie bereits festgestellt ${ }^{372}$, setzt die Einordnung als Verhaltenskodexes i. S. d. UWG im Gegensatz zur Definition der Wettbewerbsregel aus $\int$ Abs. 24 Abs. 2 GWB nicht voraus, dass die in ihm enthaltenen Regelungen die Lauterkeit im Wettbewerb oder den leistungsgerechten Wettbewerb betreffen. Entsprechend können Verhaltenskodizes aus dem ökologischen, ethischen und sozialen Bereich nicht als Wettbewerbsregeln anerkannt werden. Zudem werden Wettbewerbsregeln i. S. d. GWB nach \ 24 Abs. 1 UWG immer von Wirtschafts- oder Berufsvereinigungen aufgestellt, während der Großteil der Verhaltenskodizes aus unternehmenseigenen Verhaltenskodizes besteht. ${ }^{373}$ Insofern ist das Verhältnis zwischen Verhaltenskodizes i. S. d. UWG zu Wettbewerbsregel i. S. d. GWB das von Oberbegriffen zu Unterbegriffen.

\section{G. Zusammenfassung}

Für die Einordnung als Verhaltenskodex i. S. d. UWG darf nicht allein auf den Wortlaut der Definition in $\int 5$ Abs. 1 Nr. 5 UWG abgestellt werden. Insbesondere kann nicht die Verpflichtung zu jedem Verhalten ausreichen, sondern es muss

$366 \$ 26$ Abs. 1 Satz 2 GWB.

367 Sosnitza in: FS für Bechtold, S. 519.

368 Zur Definition der Wettbewerbsregel aus $₫ 24$ Abs. 2 GWB siehe Fn. 353.

369 Bornkamm in: Köhler/Bornkamm, UWG, \5, Rn. 163, zustimmend hierzu Nordemann, Rn. 368, der unter Verweis auf Bornkamm von einem nicht maßgeblichen Unterschied spricht.

370 Bornkamm in: Köhler/Bornkamm, UWG, \5, Rn. 166.

371 Sosnitza in: FS für Bechtold, S. 515, der Wettbewerbsregeln i. S. d. GWB als "die Spitzee des Eisbergs" und "kleinen Ausschnitt aller möglichen Verbandsregelungen" bezeichnet.

372 Vgl. Kapitel 3C.

373 Vgl. Kapitel 1C.I. 
eine Verhaltensverpflichtung vorliegen, die dem Verständnis der Richtliniengeber der UGP-Richtlinie vom Inhalt eines Verhaltenskodexes entspricht. Gegenstand eines Verhaltenskodexes muss somit entsprechend dem Begriffsverständnis im allgemeinen Sprachgebrauch die Verpflichtung eines Unternehmens auf ein auf Dauer angelegtes Verhalten sein, das sich an den Werten der Gesellschaft orientiert und im Regelfall über die gesetzlichen Anforderungen hinausgeht. Dahingegen besteht keine ungeschriebene Voraussetzung, dass sich die Verpflichtung auf das lautere Verhaltens des Unternehmens im Wettbewerb beziehen muss. Somit reichen auch Verpflichtungen aus dem ökologischen, ethischen oder sozialen Bereich aus. 374

Darüber hinausgehende Anforderungen an den Inhalt von Verhaltenskodizes bestehen nicht. Insbesondere müssen keine Regelungen zur Überwachung und Einhaltung der mit dem Verhaltenskodex eingegangenen Verpflichtung enthalten sein. Hinsichtlich des Umfangs der Verpflichtung reicht es für die Annahme eines Verhaltenskodexes i. S. d. UWG aus, dass sich der Unternehmer verpflichtet, sich zu bemühen, ein bestimmtes Verhalten einzuhalten. Entsprechend können auch Absichtserklärungen Verhaltenskodizes i. S. d. UWG sein. Gleiches gilt für deklaratorische Erklärungen, da das negative Tatbestandsmerkmal der fehlenden Vorgabe der Verpflichtung durch Gesetzes- oder Verwaltungsvorschriften im Bezug auf diese nicht zu einem Ausschluss führen darf.

Trotz der Verwirklichung des Tatbestandes von $\int 1$ GWB beziehungsweise von Art. 101 Abs. 1 AEUV stellen Verhaltenskodizes i. S. d. UWG in aller Regel keine unzulässigen Kartellvereinbarungen dar. Denn diese unterfallen, soweit es sich um Verhaltenskodizes zur Festlegung der fachlichen Sorgfalt in einer Branche handelt, bereits nicht dem Schutzzweck des GWB. Verhaltenskodizes mit ökologischen, ethischen oder sozialen Verpflichtungen sind so genannte freigestellte Vereinbarungen gemäß $₫ 2$ Abs. 1 GWB.

374 A. A. Dreyer, WRP 2007, 1294,1296, Büllesbach, S. 43. 


\section{Kapitel 4}

\section{Anwendbarkeit des UWG}

Grundvoraussetzung für eine Beurteilung von Verhaltensweisen im Zusammenhang mit Verhaltenskodizes nach dem UWG ist selbstverständlich zunächst dessen Anwendbarkeit. Aus diesem Grund werden nachfolgend in der gebotenen Kürze die sachlichen, örtlichen und persönlichen Anwendungsvoraussetzungen des UWG unter Berücksichtigung der Besonderheiten von Verhaltensweisen im Zusammenhang mit Verhaltenskodizes dargestellt.

\section{A. Sachliche Anwendbarkeit}

Sachlich ist das UWG immer dann anwendbar, wenn eine geschäftliche Handlung vorliegt. Denn das UWG dient entsprechend seinem in \$1 S. 1 festgeschriebenen Zweck dem Schutz vor unlauteren geschäftlichen Handlungen. Die Einordnung einer Verhaltensweise als geschäftliche Handlung ist damit erste und unabdingbare Voraussetzung für ihre Unlauterkeit nach dem UWG. Aus diesem Grund handelt es sich bei dem Begriff der geschäftlichen Handlung — neben dem der Unlauter- 
keit - um den Zentralbegriff des UWG. ${ }^{375}$ Denn erst nachdem eine Verhaltensweise als geschäftliche Handlung eingeordnet wurde, stellen sich die Fragen nach ihrer Lauterkeit und Zulässigkeit. Obwohl diese Einordnung in der konkreten Rechtsanwendung natürlich für jeden Einzelfall geprüft werden muss, werden dieser Arbeit die nachfolgend dargestellten fünf typischen Verhaltensweisen im Zusammenhang mit Verhaltenskodizes zugrundlegt:

1. Ein Unternehmen gibt sich einen Verhaltenskodex, ohne dies und diesen öffentlich bekannt zu machen und verwendet diesen beispielsweise als interbetriebliche Verhaltensmaßgabe.

2. Ein Unternehmen gibt sich einen Verhaltenskodex und veröffentlicht diesen beispielsweise im Internetauftritt des Unternehmens oder als Pressemitteilung.

3. Ein Unternehmen gibt sich einen Verhaltenskodex und unternimmt Marketingmaßnahmen mit dem ausschließlichen Inhalt, auf das Vorliegen und/oder den Inhalt des Verhaltenskodexes hinzuweisen.

4. Ein Unternehmen gibt sich einen Verhaltenskodex und weist im Rahmen der üblichen (Produkt)Werbung auf diesen und/oder seinen Inhalt hin.

5. Ein Unternehmen weist im Rahmen einer individuellen Ansprache gegenüber einem oder mehreren (potenziellen) Kunden auf seinen Verhaltenskodex und/oder dessen Inhalt hin.

I. Grundsätzliches zur geschäftlichen Handlung

Der Begriff der geschäftlichen Handlung selbst wurde im Jahr 2008 im Rahmen der Umsetzung der UGP-Richtlinie ${ }^{376}$ in das UWG eingeführt ${ }^{377}$ und in $\ 2$ Abs. 1 Nr. 1 UWG legaldefiniert als, ,jedes Verbalten einer Person zugunsten des eigenen oder eines fremden Unternehmens vor, bei oder nach einem Geschäftsabschluss, das mit der Förderung des Absatzes oder des Bezugs von Waren oder Dienstleistungen oder mit dem Abschluss oder der Durchfübrung eines Vertrags über Waren und Dienstleistungen objektiv zusammenbängt". Grundlage dieser Begriffsbestimmung war die Definition der Geschäftspraktik durch die UGP-Richtlinie. 378 Allerdings wurde der Begriff der Geschäftspraktik

375 Köhler in: Köhler/Bornkamm, UWG, \$ 2, Rn. 3.

376 Regierungsentwurf und Begründung zum UWG vom 20.08.2008, BT.-Drs. 16/10145, S. 20.

377 Er ersetzte den mit dem UWG 2004 eingeführten Begriff der „Wettbewerbshandlung “, welcher den bis dahin gültigen Begriff der „Handlung im geschäftlichen Verkebr zu Zwecken des Wettbewerbs" ersetzt hatte.

378 Art. 2 lit. d) UGP-Richtlinie: „,,Geschäftspraktiken im Geschäftsverkehr zwischen Unternehmen und Verbrauchern" (nachstehend auch "Geschäftspraktiken" genannt) jede Handlung, Unterlassung, Verbaltensweise oder Erklärung, kommerzielle Mitteilung einschließlich Werbung und Marketing eines Gewerbetreibenden, die unmittelbar mit der Absatzförderung, dem Verkauf oder der Lieferung eines Produkts an Verbraucher zusammenbängt;"“ 
aufgrund der negativen Belegung im deutschen Sprachgebrauch nicht übernommen $^{379}$ sowie einige Veränderungen ${ }^{380}$ und Erweiterungen ${ }^{381}$ vorgenommen. Auffälligste Erweiterung der Definition aus der UGP-Richtlinie ist die Aufnahme eines Zeitbereiches („vor, bei oder nach einem Geschäftsabschluss“). Dieser Teil der Definition stellt jedoch weniger ein Tatbestandsmerkmal der geschäftlichen Handlung, als vielmehr eine Regelung über den (zeitlichen) Anwendungsbereich des UWG dar, der ebenfalls auf der UGP-Richtlinie beruht. ${ }^{382}$ Die Übernahme dieser zeitlichen Vorgabe aus der UGP-Richtlinie war notwendig, da das UWG bis dahin im Regelfall nur Verhaltensweisen vor Vertragsabschluss erfasste. ${ }^{383}$ Bemerkenswert ist dabei, dass der deutsche Gesetzgeber diese Bestimmung des zeitlichen Anwendungsbereiches entgegen der Systematik der UGP-Richtlinie ${ }^{384}$ in die Definition der geschäftlichen Handlung aufgenommen hat. ${ }^{385}$ Denn dies hat zur Folge, dass sie für alle geschäftlichen Handlungen im Anwendungsbereich des UWG auch solche im b2b-Bereich — gilt. Hätte der deutsche Gesetzgeber die nicht von der UGP-Richtlinie erfassten Verhaltensweisen ${ }^{386}$ zwischen Unternehmern hiervon ausnehmen wollen, hätte er einen entsprechenden Passus beispielsweise in $\int 1$ UWG aufnehmen können. ${ }^{387}$ Aus der gewählten Systematik des UWG und des Wortlautes von \ 2 Abs. 1 Nr. 1 UWG lässt sich dies jedoch nicht entnehmen. ${ }^{388}$

\footnotetext{
379 Regierungsentwurf und Begründung zum UWG vom 20.08.2008, BT.-Drs. 16/10145, S. 20.

380 Während die UGP-Richtlinie konkrete Handlungsarten (Tun, Unterlassen) und Beispiele (kommerzielle Mitteilung, Werbung) benennt, beschränkt sich das UWG auf den Oberbegriff des Verhaltens. Darüber hinaus fordert die UGP-Richtlinie einen unmittelbaren Zusammenhang, während das UWG auf einen objektiven Zusammenhang abstellt.

381 Die UGP-Richtlinie bezieht sich nur auf den Absatz (vgl. Art. 2 lit. d) UGP-Richtlinie), das UWG auch auf den Bezug von Waren.

382 Art. 3 Abs. 1 UGP-Richtlinie regelt dies jedoch im Rahmen der Regelungen zum Anwendungsbereich der UGP-Richtlinie.

383 In Ausnahmefällen konnte auch ein Verhalten nach Vertragsschluss sanktioniert werden, wenn sich dieses eine Täuschung vor Vertragsschluss zu nutzen machte. Vgl. Sosnitza in: Piper/Ohly/Sosnitza, UWG, \2, Rn. 21.

384 In der UGP-Richtlinie wird in der Definition der Geschäftspraktik nur indirekt auf den zeitlichen Anwendungsbereich Bezug genommen, indem hinsichtlich des Tatbestandsmerkmales des unmittelbaren Zusammenhangs jeweils ein Obergriff aus dem jeweiligen Zeitraum (Absatzförderung $=$ vor, Verkauf $=$ bei, Lieferung $=$ nach) verwendet wird.

385 Entsprechend der Entscheidung des Gesetzgebers wird der zeitliche Anwendungsbereich nicht getrennt dargestellt, sondern an dieser Stelle im Rahmen des sachlichen Anwendungsbereiches.

386 Vgl. zum Anwendungsbereich der UGP-Richtlinie Kapitel 2B.I.1.a.

387 Bspw. wie folgt: „Dieses Gesetz dient dem Schutz der Verbraucherinnen und Verbraucher vor unlauteren geschäftlichen Handlungen vor, bei und nach einem Geschäftsabschluss sowie dem Schutz von Mitbewerbern und sonstigen Marktteilnehmern vor einem Geschäftsabschluss."
}

388 A. A Köhler in: Köhler/Bornkamm, UWG, \2, Rn. 32 f., der eine entsprechende Beschränkung aus dem Begriff des Geschäftsabschlusses herausliest. 
II. Voraussetzungen der geschäftlichen Handlung

Die Definition der geschäftlichen Handlung aus $₫ 2$ Abs. 1 Nr. 1 UWG lässt sich in zwei Voraussetzungen ${ }^{389}$ aufspalten.

1. Das Verhalten einer Person zugunsten des eigenen oder eines fremden Unternehmens

und

2. den objektiven Zusammenhang dieses Verhaltens mit der Förderung des Absatzes oder des Bezuges von Waren und Dienstleistungen oder mit der dem Abschluss oder der Durchführung eines Vertrages über Waren und Dienstleistungen.

Aufgrund der Vielzahl an auslegungsfähigen, ja sogar auslegungsbedürftigen Ausdrücken und Formulierungen ist die Definition des UWG - wie auch die der Definition der Geschäftspraktik in der UGP-Richtlinie — nicht aus sich heraus eindeutig und somit in Grenzbereichen nicht unproblematisch. Eine Konkretisierung und Auslegung durch Lehre und Judikative, insbesondere den EuGH, war und ist somit unbedingt geboten. Eine grundlegende (Vorab)Entscheidung des EuGH wurde jedoch noch nicht herbeigeführt, so dass im Hinblick auf die Auslegung der Judikative nur auf die Rechtsprechung deutscher Gerichte zurückgegriffen werden kann.

\section{Verbalten einer Person zugunsten des eigenen oder eines fremden Unternehmens}

Eine geschäftliche Handlung erfordert zunächst das Verhalten einer Person. Dabei ist aufgrund des Wortlautes der Definition im UWG sowie des immer gebotenen Rückgriffs auf die UGP-Richtlinie unerheblich, ob es sich um eine natürliche oder eine juristische Person handelt. Denn die Definition der Geschäftspraktik in der UGP-Richtlinie erfasst das Verhalten eines "Gewerbetreibenden“, worunter die

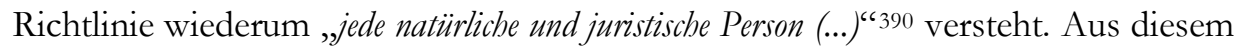
Grund verstößt eine Beschränkung des Wortlautes auf natürliche Personen gegen das Gebot der richtlinienkonformen Auslegung. ${ }^{391}$

Darüber hinaus muss das Verhalten ,zugunsten des eigenen oder eines fremden Unternehmens" erfolgen. Hierbei muss auf den von der Rechtsprechung entwickelten lauterkeitsrechtlichen Unternehmensbegriff abgestellt werden, da das UWG den

389 Das ebenfalls als Tatbestandsmerkmal formulierte zeitliche Kriterium wird dabei außen vor gelassen.

${ }^{390}$ Vgl. zur vollständigen Definition des Gewerbetreibenden nach Art. 2 lit. b) UGP-Richtlinie Fn. 295.

391 A. A. Beater, Unlauterer Wettbewerb, Rn. 882, der seine Einschränkung mit der Passivlegitimation begründet, da juristische Personen nicht als solche handeln könnten. Aus diesem Grund könne Ihnen das Verhalten natürlicher Personen nur zugerechnet werden. 
Begriff des Unternehmens zwar vielfach verwendet ${ }^{392}$, ihn im Gegensatz zum Begriff des Unternehmers ${ }^{393}$ jedoch nicht legaldefiniert. Die Unterscheidung ist jedoch wesentlich, da der Begriff des Unternehmens die betriebliche Tätigkeit und Organisation umfasst, der des Unternehmers dahingegen allein den Inhaber des Unternehmens. Die Rechtsprechung legt den Begriff des Unternehmens weit aus ${ }^{394}$ und fordert lediglich ,eine auf eine gewisse Dauer angelegte, selbständige wirtschaftliche Betätigung, die darauf gerichtet ist, Waren oder Dienstleistungen gegen Entgelt zu vertreiben." 395 Unerheblich für die Einordnung als Unternehmen ist dahingegen die Rechtsform der Organisation. ${ }^{396}$

Hinsichtlich der umfassten Verhaltensweisen beschränkt sich die Definition des UWG im Gegensatz zur Vorlage aus Art. 2 lit. d) UGP-Richtlinie, welche neben der Handlung und dem Unterlassen mit der Erklärung und der kommerziellen Mitteilung einschließlich Werbung und Sponsoring zusätzlich noch klassischerweise vom Lauterkeitsrecht umfasste Verhaltensweisen anführt, auf den Oberbegriff des Verhaltens. Mit dessen Verwendung anstelle des im definierten Begriff der geschäftlichen Handlung sowie in der Vorlage aus der UGP-Richtlinie enthaltenen Begriffs der Handlung wollte der Gesetzgeber hervorheben, dass als geschäftliche Handlung gleichermaßen ein positives Tun wie auch ein Unterlassen in Betracht kommt. ${ }^{397}$ Dabei war dem Gesetzgeber bewusst, dass der Begriff der Handlung in der deutschen Rechtsterminologie 398 ebenfalls beide Verhaltensweisen umfasst. ${ }^{399}$ Obwohl vom deutschen Gesetzgeber nicht übernommen, stellen die von der UGP-Richtlinie benannten Verhaltensweisen aufgrund des Gebots der richtlinienkonformen Auslegung Beispiele des Verhaltens zugunsten eines Unternehmens dar. Während der Begriff der Erklärung dabei entsprechend seiner Wortbedeutung eindeutig als Mitteilung beziehungsweise (offizielle) Äußerung bestimmt werden kann ${ }^{400}$, ist zum Umfang der kommerziellen Mitteilung zunächst festzustellen, dass diese — laut dem Wortlaut UGP-Richtlinie — Werbung und Marketing umfasst. Entsprechend lässt sich eine Verbindung zur kommerziellen Kommunikation aus der E-Commerce-Richtlinie herstellen. Diese umfasst „alle Formen der Kommunikation, die der unmittelbaren oder mittelbaren Förderung des Absatzes

\footnotetext{
392 So bspw. in $\int 2$ Abs. 1 Nr. 1, \4 Nr. 8, \8 Abs. 2 und $₫ 17$.

393 \ 2 Abs. 1 Nr. 6 UWG definiert den Unternehmer als ,jede natürliche und juristische Person, die geschäftliche Handlungen im Rahmen ibrer gewerblichen, handwerklichen oder beruflichen Tätigkeit vornimmt, und jede Person, die im Namen oder Auftrag einer solchen Person handelt;"“.

${ }^{394}$ Köhler in: Köhler/Bornkamm, UWG, \2, Rn. 21.

395 Urteil des BGH v. 04.12.2008 — I ZR 3/06 (OLG Frankfurt a. M.) (Ohrclips) in GRUR 2009, $871,872$.

396 Köhler in: Köhler/Bornkamm, UWG, \2, Rn. 28.

${ }^{397}$ Regierungsentwurf und Begründung zum UWG vom 20.08.2008, BT.-Drs. 16/10145, S. 20.

398 Handlung $=$ Tun und Unterlassen, vgl. Köhler in: Köhler/Bornkamm, UWG, \ 2, Rn. 10.

${ }^{399}$ Regierungsentwurf und Begründung zum UWG vom 20.08.2008, BT.-Drs. 16/10145, S. 20.

${ }^{400}$ Vgl. http://www.duden.de/rechtschreibung/Erklaerung.
} 
von Waren und Dienstleistungen oder des Erscheinungsbildes eines Unternehmens, einer Organisation oder einer natürlichen Person dienen, die eine Tätigkeit in Handel, Gewerbe und Handwerk oder einen reglementierten Beruf ausübt." "401 Die kommerzielle Kommunikation umfasst somit - wie die kommerzielle Mitteilung - Verhaltensweisen, die mit der Absatzförderung (Werbung im klassischen Sinne) beziehungsweise dem Ansehen einer Person oder Organisation (Imagewerbung, Sponsoring) zusammenhängen. Aufgrund dieser Übereinstimmung ist Köbler402 dahingehend zuzustimmen, dass die kommerzielle Mitteilung inhaltlich gleichbedeutend mit dem Begriff der kommerziellen Kommunikation ist. ${ }^{403}$ Dies entspricht im Ergebnis auch dem Willen der Richtliniengeber sowie des deutschen Gesetzgebers, welche die Imagewerbung und Sponsoring ausdrücklich als Verhalten i. S. d. UWG ${ }^{404}$ beziehungsweise als Marketingmaßnahme i. S. d. UGP-Richtlinie ${ }^{405}$ ansehen.

Zugunsten eines Unternehmens erfolgt ein solches Verhalten nicht nur dann, wenn es dem Unternehmen tatsächlich (wirtschaftlich) zugute kommt. Vielmehr reicht hierfür jeder Unternehmensbezug des Verhaltens aus. ${ }^{406}$ Dieses Merkmal dient somit insbesondere zum Ausschluss der Anwendung des UWG auf Handlungen von Privatpersonen zu privaten Zwecken sowie von Handlungen solcher Organisationen, die kein Unternehmen darstellen. 407 Hierbei handelt es sich beispielsweise um Idealvereine und Stiftungen. Gleichfalls keinen Unternehmensbezug haben dienstliche (amtlich-hoheitliche) Handlungen. ${ }^{408}$ Das Merkmal des Verhaltens zugunsten eines Unternehmens nimmt damit inhaltlich das Tatbestandsmerkmal der „Handlung im geschäftlichen Verkehr" auf, das im UWG 1909 noch explizit benannt war. ${ }^{409}$ Dieses war weit auszulegen ${ }^{410}$ und erforderte nichts weiter, „als daß irgendeine der Förderung eines Geschäftszweckes dienende Tätigkeit im Gegensatz, zu einer rein privaten oder amtlichen Betätigung vorliege. "“11 Obwohl das UWG 2004 das Tatbestandsmerkmal der „Handlung im geschäftlichen Verkehr“ im Zuge der Novellierung nicht in den neuen Begriff der Wettbewerbshandlung übernahm, wurde es doch weiterhin für deren Annahme vorausgesetzt. ${ }^{412}$ Aus diesem Grund erscheint es angebracht, die Voraussetzungen der „Handlung im gescbäftlichen Verkehr“

\footnotetext{
401 Art. 2 lit. f) der E-Commerce-Richtlinie.

402 Köhler in: Köhler/Bornkamm, UWG, \2, Rn. 14.

${ }^{403}$ Emmerich, \4, Rn. 2.

${ }^{404}$ Regierungsentwurf und Begründung zum UWG vom 20.08.2008, BT.-Drs. 16/10145, S. 21.

405 Vgl. Kapitel 4A.II.2.a.

406 Köhler in: Köhler/Bornkamm, UWG, 『2, Rn. 17.

407 Köhler in: Köhler/Bornkamm, UWG, \2, Rn. 18.

408 Sosnitza in: Piper/Ohly/Sosnitza, UWG, $\ 2$, Rn. 13.

${ }^{409}$ Das UWG 2009 verwendete anstelle der „geschäftlichen Handlung“ den Begriff der „Handlung im geschäftlichen Verkehr zu Zwecken des Wettbewerbs".

${ }^{410}$ Sosnitza in: Piper/Ohly/Sosnitza, UWG, \2, Rn. 8.

411 Urteil des BGH v. 17.03.1953 - I ZR 118/52 (OLG Düsseldorf) in GRUR 1953, 293, 294.

412 Für das UWG 2004: Urteil des BGH vom 26.01.2006 (OLG Düsseldorf) (AbschleppkostenInkasso) in GRUR 2006, 428, 428.
} 
auf das Tatbestandsmerkmal des „Verhalten zugunsten des eigenen oder eines fremden Unternebmens" zu übertragen, anstatt ersteres als ungeschriebenes Tatbestandsmerkmal parallel anzuwenden. ${ }^{413}$ Dies entspricht auch der richtlinienkonformen Auslegung. Denn der der geschäftlichen Handlung zugrundeliegenden Begriff der "Geschäftspraktike" aus der UGP-Richtlinie lautet korrekt und in voller Länge „Geschäftspraktiken im Geschäftsverkebr zwischen Unternehmen und Verbrauchern". ${ }^{414}$ Die Verkürzung auf den Begriff der Geschäftspraktik dient nur dem besseren Verständnis und der einfacheren Verwendung im weiteren Verlauf der UGPRichtlinie. Die UGP-Richtlinie nimmt somit eine Terminologie des UWG 1909 nur leicht verändert ${ }^{415}$ auf, welche in Deutschland seit dem UWG 2004 verschwunden war. Voraussetzung einer "Handlung im geschäftlichen Verkehr" und damit auch eines „Verbalten zugunsten des eigenen oder eines fremden Unternehmens" ist somit, dass das Verhalten in der Außenwelt in Erscheinung tritt und sich auf gegenwärtige oder mögliche Mitbewerber auswirken kann. ${ }^{416}$ Aus diesem Grund spricht man auch vom Außenbezug einer Handlung. ${ }^{417}$ Dies entspricht auch dem Sinn und Zweck der UGP-Richtlinie, da diese nach dem Willen des Richtliniengebers nur solche Geschäftspraktiken regeln soll, ,die in unmittelbarem Zusammenhang mit der Beeinflussung der geschäftlichen Entscheidungen des Verbrauchers in Bezug auf Produkte stehen." ${ }^{418}$ Eine Beeinflussung kann jedoch nur dann vorliegen, wenn der Verbraucher Kenntnis von dem zu beurteilenden Verhalten erlangen kann. Denn andernfalls kann dieses die Entscheidung des Verbrauchers auch nicht beeinflussen.

Dementsprechend scheidet nur für Verhaltensweisen im Zusammenhang mit Verhaltenskodizes nach Nr. 1 der Auflistung die Annahme einer geschäftlichen Handlung aufgrund eines fehlenden Außenbezuges aus, da in diesen Fällen keine Handlung zugunsten eines Unternehmens vorliegt. Dass sich entsprechende innerbetriebliche Weisungen selbstverständlich mittelbar auf den Wettbewerb auswirken (können), reicht für die Anwendung des UWG nicht aus. ${ }^{419}$ Denn dies würde einen erheblichen Eingriff in den geschützten Kernbereich der unternehmerischen Tätigkeit darstellen, der mit dem verfolgten Schutzzweck des UWG nicht gerechtfertigt werden kann. Dies insbesondere im Hinblick darauf, dass die Anwendung des Lauterkeitsrechts für entsprechende Verhaltensweisen nur aufgeschoben, aber nicht aufgehoben ist. Denn das Verhalten kann in dem Moment auf

\footnotetext{
${ }^{413}$ Sosnitza in: Piper/Ohly/Sosnitza, UWG, $\$ 2, R n .7$.

414 Art. 2 lit. d) UGP-Richtlinie.

415 Geschäftsverkehr (UGP-Richtlinie) gegenüber geschäftlicher Verkehr (UWG 1909).

416 Urteil des BGH vom 15.09.1970 - I ZR 47/69 (OLG München) (Branchenverzeichnis) in GRUR 1971, 119.

417 Beater, Rn. 883.

418 Erwägungsgrund 7 der UGP-Richtlinie.

419 Beater, Rn. 883.
} 
Anwendbarkeit des UWG

seine Lauterkeit überprüft werden, in welchem es Außenwirkung erlangt. ${ }^{420}$ Bei den unter den Nr. 2 bis 5 benannten Verhaltensweisen ist die Voraussetzung des Verhaltens einer Person zugunsten des eigenen oder eines fremden Unternehmens dahingegen erfüllt.

\section{Objektiver Zusammenhang mit der Förderung des Absatzes oder des Bezuges von $W$ aren oder Dienstleistungen oder mit dem Abschluss oder der Durchführung eines $V$ ertrages über $W$ aren und Dienstleistungen}

Die Annahme einer geschäftlichen Handlung nach dem UWG ${ }^{421}$ setzt weiter voraus, dass ein objektiver Zusammenhang besteht zwischen dem Verhalten einer Person zugunsten des eigenen oder fremden Unternehmens ${ }^{422}$ und der Förderung des Absatzes oder des Bezuges von Waren und Dienstleistungen oder der Durchführung eines Vertrages über Waren oder Dienstleistungen.423 Dieses Tatbestandsmerkmal erscheint auf den ersten Blick deutlich komplexer als sein Pendant in der UGP-Richtlinie. ${ }^{424}$ Dies liegt insbesondere an der Entscheidung des deutschen Gesetzgebers, die Definition des Begriffs „Produkt“, welcher von der UGP-Richtlinie getrennt definiert wird ${ }^{425}$, in die Definition der geschäftlichen Handlung aufzunehmen. Grund hierfür war, dass der deutsche Gesetzgeber den Begriff „Produkt" nicht verwenden wollte, da dieser dem deutschen Wettbewerbsrecht fremd ist. ${ }^{426}$ Darüber hinaus hat der deutsche Gesetzgeber nicht nur den Absatz von Produkten geregelt, sondern auch deren Bezug. ${ }^{427}$ Dies erklärt sich aus dem weiteren Anwendungsbereich des UWG. Denn in dem von der UGPRichtlinie geregelten Rechtsverhältnis zwischen Unternehmer und Verbraucher wird der Unternehmer nur in absoluten Ausnahmefällen Produkte von einem Verbraucher beziehen.

Trotz dieser Änderungen des deutschen Gesetzgebers bleibt eindeutig bestimmbar, womit ein objektiver Zusammenhang bestehen muss. Weniger eindeutig ist jedoch, wann ein solcher besteht. Hierbei ist zu beachten, dass sich der

${ }^{420}$ So hat der BGH in seinem Branchenverzeichnisurteil vom 15.09 .1970 (vgl. Fn. 416) bzgl. einer in einer innerbetrieblichen Anweisung verwendeten wettbewerbswidrigen Werbeaussage deutlich gemacht, dass sich die lauterkeitsrechtliche Beurteilung in dem Moment ändert, in welchem diese Werbeaussage außerhalb des Unternehmens verwendet wird.

$421 \rrbracket 2$ Abs. 1 Nr. 1 UWG.

422 Vgl. Kapitel 4A.II.1.

${ }^{423}$ Als Waren gelten dabei auch Grundstücke, als Dienstleistungen auch Rechte und Verpflichtungen, $₫ 2$ Abs. 1 Nr. 1 UWG.

${ }^{424}$ Der „unmittelbare Zusammenhang" mit „der Absatsförderung, dem Verkauf oder der Lieferung eines Produkts" aus Art. 2 lit. d) UGP-Richtlinie.

425 Art. 2 lit. c) UGP-Richtlinie: „,,Produkt“ jede Ware und Dienstleistung, einscbließlich Immobilien, Rechte und Verpflichtungen."

426 Regierungsentwurf und Begründung zum UWG vom 20.08.2008, BT.-Drs. 16/10145, S. 12.

427 Begründung der Bundesregierung für den Gesetzentwurf des UWG vom 22.08.2003, BT.Drs. 15/1487, S. 16, Köhler in: Köhler/Bornkamm, UWG, \2, Rn. 38. 
deutsche Gesetzgeber bewusst für den gegenüber dem „unmittelbaren Zusammenbang" inhaltlich weiteren Begriff des „objektiven Zusammenhangs" entschieden hat, da die Übernahme des Tatbestandsmerkmales des ,unmittelbaren Zusammenhangs" nach seiner Ansicht nicht mit dem gegenüber der UGP-Richtlinie weiteren Schutzbereich des UWG ${ }^{428}$ vereinbar war. ${ }^{429}$ Hintergrund war, dass das UWG auch weiterhin nicht nur für geschäftliche Handlungen von Unternehmern gegenüber Verbrauchern (b2c-Bereich), sondern auch für solche zwischen zwei Unternehmen (b2b-Bereich) gelten sollte und der Gesetzgeber für unlautere Verhaltensweisen gegenüber Mitbewerbern wie beispielsweise Absatz- und Wettbewerbsbehinderungen oder Betriebsstörungen (wie Betriebsspionage) in der Regel keinen solchen unmittelbaren Zusammenhang mit dem Absatz oder Bezug von Waren und Dienstleistungen erkannte.430 Aufgrund des Gebots der richtlinienkonformen Auslegung des UWG ist diese Änderung der Begrifflichkeit jedoch insoweit ohne Belang, als Verhaltensweise im b2c-Verhältnis zu beurteilen sind. In diesen Fällen bleibt allein die Bedeutung des Begriffs des unmittelbaren Zusammenhangs maßgeblich. ${ }^{431}$ Grund hierfür ist die mit der UGP-Richtlinie angestrebte Vollharmonisierung des europäischen Lauterkeitsrechts, die eine einheitliche Auslegung des mit der UGP-Richtlinie geregelten Bereiches erfordert und keine Kürzungen und Erweiterungen zulässt. ${ }^{432}$ Nur soweit es Verhaltensweisen im b2b-Verhältnis betrifft, werden auch die Regelungen und Wertungen des UWG in die Auslegung des Begriffs einbezogen. ${ }^{433}$ Der Begriff des objektiven Zusammenhangs ist somit für Verhaltensweisen im b2c- und b2b-Bereich unterschiedlich auszulegen.

\section{a. Objektiver Zusammenhang im b2c-Bereich}

Bei Verhaltensweisen im b2c-Bereich ist ein objektiver Zusammenhang i. S. d. UWG nur dann gegeben, wenn ein unmittelbarer Zusammenhang i. S. d. UGPRichtlinie vorliegt. Wann ein solcher unmittelbarer Zusammenhang vorliegt ist dabei mangels Vorgaben in der Regelung im Rahmen einer teleologischen und systematischen Auslegung zu ermitteln. ${ }^{434}$ Dabei kann den zu Sinn und Zweck der

428 Vgl. hierzu nachfolgend Kapitel 4C.

${ }^{429}$ Regierungsentwurf und Begründung zum UWG vom 20.08.2008, BT.-Drs. 16/10145, S. 21.

${ }^{430}$ Ein objektiver Zusammenhang sollte dahingegen gegeben sein. Denn diese Verhaltensweisen führen in aller Regel zu positiven Auswirkungen für die unlauter handelnden Unternehmen. Vgl. Regierungsentwurf und Begründung zum UWG vom 20.08.2008, BT.-Drs. 16/10145, S. 21.

${ }^{431}$ Köhler in: Köhler/Bornkamm, UWG, \2, Rn. 43, Keller in: Harte-Bavendamm/HenningBodewig, UWG, $₫ 2$ Rn. 56.

432 Vgl. Kapitel 2B.I.1.a.

${ }^{433}$ Köhler in: Köhler/Bornkamm, UWG, \2, Rn. 52.

${ }^{434}$ Eine historischen Auslegung kommt nicht in Betracht, da die UGP-Richtlinie keine direkte Vorgängerregelung hat. Zwar gab es bereits zuvor europäische Richtlinien zu Teilbereichen des Lauterkeitsrechts (so bspw. die Richtlinie des Rates vom 10. September 1984 über irreführende und vergleichende Werbung - 84/50/EWG), jedoch kein umfassendes, der UGP-Richtlinie vergleichbares Regelwerk. Darüber hinaus berührt die UGP-Richtlinie die Richtlinie über irre- 
Anwendbarkeit des UWG

Norm im Verständnis der Richtliniengeber Hinweis gebenden Erwägungsgründen diesbezüglich nur entnommen werden, dass sich die UGP-Richtlinie auf Geschäftspraktiken bezieht, „die in unmittelbarem Zusammenhang mit der Beeinflussung der geschäftlichen Entscheidungen ${ }^{435}$ des Verbrauchers in Bezug auf Produkte stehen." "436 Hieraus lässt sich jedoch nur entnehmen, womit ein Verhalten unmittelbar zusammenhängen muss — nämlich mit der Beeinflussung der geschäftlichen Entscheidungen und nicht, wann ein unmittelbarer Zusammenhang zwischen dem Verhalten und der Beeinflussung einer geschäftlichen Entscheidung vorliegt.

Ergiebiger ist dahingegen die systematische Auslegung der UGP-Richtlinie. Denn die UGP-Richtlinie regelt nach Art. 6 Abs. 1 lit. c) UGP-Richtlinie auch Geschäftspraktiken, ,„\{...\} die im Zusammenhang mit direktem oder indirektem Sponsoring stehen." Hieraus ergibt sich, dass die Richtliniengeber Sponsoring grundsätzlich als Geschäftspraktik i. S. d. UGP-Richtlinie anerkennen ${ }^{437}$ Dies bedeutet jedoch im Umkehrschluss, dass der unmittelbare Zusammenhang i. S. d. Art. 2 lit. d) UGPRichtlinie keinen unmittelbaren Kausalzusammenhang zwischen der zu beurteilenden Verhaltensweise und der Verbraucherentscheidung beziehungsweise der Absatzförderung voraussetzen kann. ${ }^{438}$ Denn ein solcher Kausalzusammenhang liegt bei Sponsoringmaßnahmen nicht vor, da diese weder einen unmittelbaren Einfluss auf die Entscheidung des Verbrauchers haben noch aus Sicht des Unternehmens unmittelbar absatzfördernd wirken sollen. ${ }^{439}$ Vielmehr sind Sponsoringmaßnahmen eher mit dem Instrument der Imagewerbung vergleichbar. Wie diese bewerben sie nicht ein konkretes Produkt oder eine konkrete Dienstleistung, sondern dienen dazu, den Bekanntheitsgrad der sponsernden Unternehmen zu erhöhen beziehungsweise die positiven Verbindungen des Publikums mit der gesponserten Veranstaltung oder Person auf das sponsernde Unternehmen zu übertragen. Letztendlich soll dies natürlich zu einer Absatzförderung führen, jedoch eben nur mittelbar (über die höhere Bekanntheit, das bessere Image) und nicht unmittelbar. Für eine solche weitere Auslegung des unmittelbaren Zusam-

führende und vergleichende Werbung ausdrücklich nicht, soweit diese vergleichende Werbung und Werbung, die für Unternehmen, aber nicht für Verbraucher irreführend ist, betrifft (Erwägungsgrund 6 der UGP-Richtlinie). Dies hat seinen Grund im weiteren Anwendungsbereich der Richtlinie 84/50/EWG, die nicht nur auf Verhaltensweisen von Unternehmern ggü. Verbrauchern anwendbar ist, sondern auch auf solche zwischen Unternehmen.

435 Der Begriff der geschäftlichen Entscheidung wird in Art. 2 lit. k) UGP-Richtlinie definiert als ,jede Entscheidung eines Verbrauchers darïber, ob, wie und unter welchen Bedingungen er einen Kauf tätigen, eine Zablung insgesamt oder teilweise leisten, eine Produkt behalten oder abgeben oder ein vertragliches Recht im Zusammenhang mit dem Produkt ausüben will, unabbängig davon, ob der Verbraucher beschließt, tätig ₹u werden oder ein Tätigwerden zu unterlassen.“

436 Erwägungsgrund 7 der UGP-Richtlinie.

${ }^{437}$ Als Unterfall einer Marketingmaßnahme, die wiederum eine kommerzielle Mitteilung darstellt, vgl. Art. 2 lit d) UGP-Richtlinie.

438 Köhler, WRP 2007, 1393, 1395.

$439 \mathrm{Vgl}$. Fn. 438. 
menhanges spricht zudem auch die Verwendung des Begriffs der kommerziellen Mitteilung 440 in Art. 2 lit. d) UGP-Richtlinie, der sowohl alle unmittelbaren als auch alle mittelbaren Mitteilungen, die der Förderung des Absatzes oder des Erscheinungsbilds eines Unternehmens dienen, umfasst ${ }^{441}$ und ansonsten eines erheblichen Teils seiner Bedeutung beraubt würde.

Aus diesem Grund besteht nicht nur bei der Produktwerbung (Verhaltensweise Nr. 4.) sowie bei der individuellen Ansprache eines Verbrauchers (Verhaltensweise Nr. 5.) ein unmittelbarer Zusammenhang mit der Absatzförderung, sondern auch bei der mit dem Sponsoring vergleichbaren Imagewerbung (Verhaltensweise Nr. 3) Weniger eindeutig ist die Annahme eines solchen unmittelbaren Zusammenhangs dahingegen für die Verhaltensweisen entsprechend der Nr. 2. Dies insbesondere, da sich die UGP-Richtlinie ausweislich der Erwägungsgründe ${ }^{442}$ nicht auf Verhaltensweisen ${ }^{443}$ beziehen soll, ,die vorrangig anderen Zwecken (als der Beeinflussung der geschäftlichen Entscheidungen von Verbrauchern ${ }^{444}$, Anmerkung d. Verf.) dienen." Als Beispiele 445 für solche Verhaltensweisen nennt die UGP-Richtlinie ,kommerzielle, für Investoren gedachte Mitteilungen, wie Jahresberichte und Unternehmensprospekte“. 446 Im Gegensatz zu den von der UGP-Richtlinie benannten Beispielen erfolgt die Veröffentlichung eines Verhaltenskodexes jedoch nicht vorrangig zu anderen Zwecken als der Beeinflussung von geschäftlichen Entscheidungen. Denn auch wenn es verschiedene Motivationen zur Abgabe eines Verhaltenskodexes gibt, so erfolgt die Information über diese doch vorrangig aus wirtschaftlichen Gründen. Aus diesem Grund ist auch für diese Verhaltensweisen ein unmittelbarer Zusammenhang i. S. d. Art. 2 lit. d) UGP-Richtlinie feststellen.

440 Zur inhaltlichen Gleichsetzung des Begriffs der kommerziellen Mitteilung zum Begriff der kommerziellen Kommunikation vgl. Kapitel 4A.II.1.

${ }^{441}$ Köhler in: Köhler/Bornkamm, UWG, \$ 2, Rn. 47.

${ }^{442}$ Vgl. Fn. 436.

${ }^{443}$ Die UGP-Richtlinie spricht an der nachfolgend zitierten Textstelle nicht von Verhaltensweisen, sondern von Geschäftspraktiken. Hierbei handelt es sich jedoch um eine terminologische Ungenauigkeit. Denn in Erwägungsgrund 7 geht es ja gerade um die Begründung, welche Verhaltensweisen der UGP-Richtlinie unterfallen sollen und welche nicht. Wären alle in Erwägungsgrund 7 als Geschäftspraktik bezeichnete Verhaltensweisen — wie beispielsweise Unternehmensprospekte - Geschäftspraktiken i. S. d. UGP-Richtlinie, wäre die Richtlinie zwangsläufig auch auf diese anwendbar. Dies soll ja aber gerade ausgeschlossen werden.

444 Erwägungsgrund 7 der UGP-Richtlinie.

445 Wie die UGP-Richtlinie benennt auch der deutsche Gesetzgeber Verhaltensweisen, die nicht dem UWG unterfallen. Allgemein sind dies solche Verhaltensweisen, die in keinem objektiven Zusammenhang mit dem Absatz oder Bezug von Waren und Dienstleistungen oder den Fällen der horizontalen Behinderung stehen. So beispielsweise weltanschauliche, wissenschaftliche, redaktionelle oder verbraucherpolitische Äußerungen oder die Reichweitenforschung, vgl. Regierungsentwurf und Begründung zum UWG vom 20.08.2008, BT.-Drs. 16/10145, S. 21.

446 Erwägungsgrund 7 der UGP-Richtlinie. 
b. Objektiver Zusammenhang im b2b-Bereich

Anders als bei Verhaltensweisen im b2c-Bereich ist das Tatbestandsmerkmal des objektiven Zusammenhangs bei Verhaltensweisen im b2b-Bereich entsprechend dem Willen des deutschen Gesetzgebers auszulegen. ${ }^{447}$ Da dieser mit dem Abstellen auf einen objektiven Zusammenhang den Anwendungsbereich erweitern wollte, sind zunächst sämtliche Verhaltensweisen umfasst, bei welchen ein unmittelbarer Zusammenhang i. S. d. UGP-Richtlinie vorliegt. ${ }^{448}$ Unter Rückgriff auf den Willen des deutschen Gesetzgebers liegt ein objektiver Zusammenhang zudem bei Fällen der horizontalen Behinderung wie Absatz- und Werbebehinderungen, Betriebsstörungen (etwa Betriebsspionage) sowie bei unberechtigten Abmahnungen vor. ${ }^{449}$ Denn auch diese Verhaltensweisen wirken sich letztlich — wenn auch mit einer zeitlichen Verzögerung — zugunsten des handelnden Unternehmens aus. ${ }^{450}$

Grundsätzlich ist es für die Annahme eines objektiven Zusammenhangs i. S. d. \ 2 Abs. 1 Nr. 1 UWG ausreichend, aber auch notwendig, dass das Verhalten objektiv geeignet ist, den Absatz oder den Bezug von Waren oder Dienstleistungen oder den Abschluss oder die Durchführung eines Vertrages über Waren und Dienstleistungen zu fördern. Nicht erforderlich ist dahingegen, dass es tatsächlich zu einer Förderung kommt. ${ }^{451}$ Ein diesbezüglich objektiv untaugliches Verhalten reicht damit nicht aus, auch wenn der Handelnde die Förderung der benannten Zwecke beabsichtigt. ${ }^{452}$ Abweichend von der vorherigen Rechtslage verzichtet das UWG 2008 somit vollständig auf ein subjektives Element, da dies dem deutschen Gesetzgeber als nicht mit Art. 2 lit. d) UGP-Richtlinie vereinbar erschien. ${ }^{453}$ Zudem sollte durch die rein objektive Bewertung die Rechtssicherheit gefördert werden. ${ }^{454}$

\section{B. Örtliche Anwendbarkeit}

Die Frage der örtlichen Anwendbarkeit des UWG bedarf bei Sachverhalten im Zusammenhang mit Verhaltenskodizes einer besonderen Beachtung, da diese oftmals einen internationalen Bezug aufweisen ${ }^{455}$ und damit die Anwendung der

\footnotetext{
447 Köhler in: Köhler/Bornkamm, UWG, \2, Rn. 52.

448 Somit liegt bei den Verhaltensweisen im Zusammenhang mit Verhaltenskodizes entsprechend der Nummern 2. bis 5. auch ein objektiver Zusammenhang i. S. d. \2 Abs. 1 Nr. 1 UWG vor.

$449 \mathrm{Vgl}$. Fn. 450.

450 Regierungsentwurf und Begründung zum UWG vom 20.08.2008, BT.-Drs. 16/10145, S. 21.

451 Köhler in: Köhler/Bornkamm, UWG, \2, Rn. 37.

452 Köhler, WRP 2007, 1393, 1394.

453 Regierungsentwurf und Begründung zum UWG vom 20.08.2008, BT.-Drs. 16/10145, S. 20 f.

454 Sosnitza in: Piper/Ohly/Sosnitza, UWG, \2, Rn. 24.

455 So beispielsweise, wenn das den Verhaltenskodex abgebende Unternehmen seinen Sitz im Ausland hat, die gegen den Verhaltenskodex verstoßenden Handlung im Ausland begangen wird oder ein deutsches Unternehmen im Ausland tätig wird.
} 
Rechte verschiedener Staaten in Betracht kommt. Da es sich bei Verstößen gegen das UWG um unerlaubte Handlungen handelt, ${ }^{456}$ bestimmt sich dieses sogenannte Kollisionsrecht aufgrund der „Rom II - Verordnung zur einheitlichen Regelung des internationalen Deliktsrechts in der Europäischen Union“. Diese gilt für sämtliche Mitgliedstaaten der Europäischen Union mit Ausnahme von Dänemark ${ }^{457}$, jedoch nur für Ereignisse nach dem Inkrafttreten der Verordnung am 11.01.2009.458 An der Geltung der Rom-II-Verordnung ändert auch der als Binnenmarktklausel bezeichnete Art. 4 UGP-Richtlinie nichts, wonach , die Mitgliedstaaten den freien Dienstleistungsverkehr und den freien Warenverkehr nicht aus Gründen, die mit dem durch die UGP-Richtlinie angeglichenen Bereich zusammenhängen, einschränken (dürfen, Ergänzung d. d. Verf.).“ Denn bei Art. 4 UGP-Richtlinie handelt es sich lediglich um ein Überbleibsel (vorgesehen als Art. 4 Abs. 2 des Richtlinienentwurfs ${ }^{459}$ ) der ursprünglich angedachten Regelung, mit der die Europäische Kommission versucht hatte, im Rahmen der UGP-Richtlinie das Herkunftslandprinzip (unter der neuen Begrifflichkeit „Binnenmarktprinzip“) einzuführen. ${ }^{460}$ Aufgrund des Widerstandes der Mitgliedstaaten wurde der eigentlich entscheidende Art. 4 Abs. 1 des Entwurfs der Kommission jedoch gestrichen. ${ }^{461}$ Der Widerstand der Mitgliedstaaten gründete dabei nicht zuletzt auf der bereits zum damaligen Zeitpunkt vorbereiteten Rom II - Verordnung.

Deutsche Gerichte haben somit unter Umständen nicht aufgrund des UWG $\mathrm{zu}$ entscheiden, sondern beispielsweise auf Grundlage des schweizerischen ${ }^{462}$ UWG. ${ }^{463}$ Gleichzeitig kann auch ein französisches ${ }^{464}$ Gericht verpflichtet sein, einen Sachverhalt auf Grundlage des deutschen UWG zu entscheiden. Die konkreten Regelungen zur kollisionsrechtlichen Bewertung von ,unlauterem Wettbewerbsverhalten" ${ }^{465}$ finden sich in Art. 6 Abs. 1, Abs. 2 der Rom II - Verordnung und

456 Vgl. nur Urteil des BGH v. 18.10.2001 - I ZR 22/99 (OLG Düsseldorf) (Meißner Decor) in NJW-RR 2002, 832.

457 Art. 1 Abs. 4 der Rom II-Verordnung.

${ }^{458}$ Für Ereignisse vor diesem Zeitpunkt gelten weiterhin die Regelungen der $\$ \int 40 \mathrm{ff}$. EGBGB, vgl. zur Darstellung der Rechtslage für diese Fälle Köhler in: Köhler/Bornkamm, UWG, Einl., Rn. $5.4 \mathrm{ff}$.

459 KOM (2003) 356 endgültig.

${ }^{460}$ Henning-Bodewig, GRURInt 2004, 183, 187.

${ }^{461}$ Dieser bestimmte, dass sich Gewerbetreibende ,auf dem durch diese Richtlinie angeglichenen Gebiet lediglich an die Rechtsvorschriften" des Staates ihrer Niederlassung zu halten haben, KOM (2003) 356 endgültig.

462 Die Maßgaben der Rom II - Verordnung gelten nicht nur im Verhältnis der Mitgliedstaaten untereinander, sondern haben territorial eine universelle Geltung, vgl. Köhler in: Köhler/Bornkamm, UWG, Einl., Rn. 5.28.

${ }^{463}$ Das Kollisionsrecht regelt somit nur die Frage, welches Recht anwendbar ist und nicht, welches Gericht zuständig ist.

${ }^{464}$ Für ein Schweizer Gericht gilt dies nicht, da die Schweiz kein Mitglied der Europäischen Union ist.

${ }^{465}$ Der Begriff des „unlauteren Wettbewerbsverhaltens“ ist dabei gemeinschaftsrechtlich auszulegen. 
sind für die Mitgliedstaaten verbindlich. 466 So ist gemäß deren Art. 6 Abs. 1 grundsätzlich das „Recht des Staates anquwenden, in dessen Gebiet die Wettbewerbsberiebungen oder die kollektiven Interessen der Verbraucher beeinträchtigt worden sind oder wahrscheinlich beeinträchtigt werden. "Ausnahmen hiervon gelten nach Art. 6 Abs. 2 Rom II - Verordnung nur für die Fälle, in denen „ausschließlich die Interessen eines bestimmten Wettbewverbers beeinträcbtigt (werden, Ergänzung d. d. Verf.). “467 Für diese Fälle verweist Art. 6 Abs. 2 Rom II - Verordnung auf die differenzierten Regelungen ihres Art. 4 über das Kollisionsrecht bei allgemeinen deliktischen Handlungen. 468

$\mathrm{Da}$ es sich bei den vorstehend als geschäftliche Handlungen eingeordneten Verhaltensweisen ausschließlich um markt- und nicht um konkurrentenbezogene Verhaltensweisen handelt, bestimmt sich das anzuwendende Recht für diese nach Art. 6 Abs. 1 Rom II - Verordnung. Entscheidend ist somit, auf welchem Staatsgebiet die Wettbewerbsbeziehungen oder die kollektiven Verbraucherinteressen durch die geschäftliche Handlung ${ }^{469}$ beeinträchtigt worden sind oder beeinträchtigt werden können. Dies entspricht im Wesentlichen der bereits zuvor bestehenden deutschen Rechtsprechung unter dem Stichwort Marktortsprinzip, welcher der Gedanke zugrunde liegt, dass „unlauterer Wettbewerb $\{\ldots\}$ in der Regel nur dort begangen werden (kann, Ergänzung d. d. Verf.), wo wettbewerbliche Interessen der Mitbewerber aufeinanderstoßen." 470

Ergänzend zu den Regelungen der Rom II - Verordnung bestehen im Gemeinschaftsrecht noch Sonderregelungen für sogenannte ubiquitäre geschäftliche Handlungen. ${ }^{471}$ Hierbei handelt es sich um solche geschäftliche Handlungen, die nicht an bestimmten Raum gebunden sind, sondern ihre Wirkungen an einer Vielzahl von Orten entfalten. ${ }^{472}$ Als Paradebeispiele hierfür gelten das Internet, dessen Inhalte unabhängig vom Ort der Einspeisung weltweit verfügbar sind sowie Funk und Fernsehen, welche über Satellit ebenfalls über große Distanzen und über

466 Denn Art. 6 Abs. 4 Rom II - Verordnung bestimmt, dass von Artikel 6 Rom II - Verordnung nicht durch eine Vereinbarung nach Art. 14 Rom II - Verordnung, welcher den Mitgliedstaaten grundsätzlich freie Rechtswahl gewährt, abgewichen werden kann.

${ }^{467}$ So beispielsweise im Fall von Industriespionage oder dem rechtswidrigen Abwerben von Mitarbeitern.

468 Nach dessen Abs. 1 ist zunächst grundsätzlich der Erfolgsort einer Handlung maßgeblich. Dies gilt jedoch dann nicht, wenn die geschädigte Person und der Delinquent ihren gewöhnlichen Aufenthalt in demselben Staat haben. In diesem Fall ist dessen Recht anzuwenden, Art. 4 Abs. 2 Rom II - Verordnung. Darüber hinaus kann nach Abs. 3 der Norm auch das Recht eines dritten Staates anzuwenden sein, wenn die zu beurteilende Handlung offensichtlich einen engeren $\mathrm{Zu}$ sammenhang mit diesem Staat hat als mit den Staaten entsprechend der Art. 1 und 2 Rom II Verordnung.

${ }^{469}$ Vgl. Kapitel 4A.

470 Urteil des BGH v. 30.06.1961 - I ZR 39/60 (OLG Hamburg) (Kindersaugflaschen) in BGH GRUR 1962, 243, 245.

471 Beater, Rn. 759.

$472 \mathrm{Vgl}$. Fn. 471. 
Ländergrenzen hinweg zu empfangen sind. ${ }^{473}$ Gerade für diese im Bereich der Werbung äußerst praxisrelevanten Bereiche schreibt der auf Art. 3 Abs. 2 ECommerce-Richtlinie und den Art. 2 und Art. 3 der Richtlinie über audiovisuelle Mediendienste 474 beruhende $\ 3$ TMG jedoch das Herkunftslandprinzip vor. Da der BGH eine höchstrichterliche Entscheidung hierzu ausgelassen hat 475 ist weiterhin umstritten, ob den Regelungen aus diesen Richtlinien und damit auch \ 3 TMG aufgrund der vorzunehmenden richtlinienkonformen Auslegung ein kollisionsrechtlicher Gehalt zukommt. 476

Für Art. 3 Abs. 2 E-Commerce Richtlinie muss dies insbesondere im Hinblick auf deren Art. 1 Abs. 4 und Erwägungsgrund 23 abgelehnt werden, da dieser ausdrücklich keine zusätzlichen Regelungen im internationalen Privatrecht schaffen soll. ${ }^{477}$ Dies bedeutet für den praxisrelevanten Fall der Veröffentlichung von Verhaltenskodizes im Internet, dass der Ort der wettbewerbsrechtlichen Interessenskollision überall dort ist, wo die Webseite bestimmungsgemäß aufgerufen werden kann. ${ }^{478}$ Der BGH hat es Werbenden jedoch zugestanden, das Verbreitungsgebiet ihrer Werbung im Internet durch einen sogenannten Disclaimer einzuschränken, wenn sie in diesem ankündigen, Adressaten in einem bestimmten Land nicht zu beliefern. ${ }^{479}$ Voraussetzung ist jedoch, dass der Disclaimer eindeutig gestaltet und auf Grund seiner Aufmachung als ernst gemeint aufzufassen ist und vom Werbenden auch tatsächlich beachtet wird. 480

Im Gegensatz zur E-Commerce Richtlinie ist der Regelung in der Richtlinie über audiovisuelle Mediendienste ein solcher kollisionsrechtlicher Gehalt wohl zuzusprechen, da diese keine dem Art. 1 Abs. 4 E-Commerce-Richtlinie entsprechende Regelung enthält. ${ }^{481}$ Dies hat aufgrund des begrenzten Anwendungsbereiches der Richtlinie über audiovisuelle Mediendienste jedoch nur eine eingeschränkte praktische Bedeutung. Denn dort gilt das Herkunftslandprinzip ohnehin nur für die Fernsehanbieter selbst und damit nicht für die werbenden Unternehmen und darüber hinaus auch nur für den sogenannten ,koordinierten“ Bereich und

\footnotetext{
${ }^{473}$ Vgl. Fn. 471

${ }^{474} \mathrm{Die}$ Richtlinie über audiovisuelle Mediendienste ersetzte die sog. Fernseh-Richtlinie 98/552/EWG, welche 2007 durch die Richtlinie 2007/65/EG über audiovisuelle Mediendienste grundlegend novelliert wurde.

475 Urteil des BGH v. 30.03.2006 - I ZR 24/03 (KG Berlin) (Arzneimittelwerbung im Internet) in GRUR 2006, 513, welches in Tz. 29 lediglich auf den Diskussionsstand verweist.

476 Zum Streitstand vgl. insbesondere Sack, WRP 2008, 845, 854 ff., der einen kollisionsrechtlichen Gehalt verneint.

477 Götting in: Götting/Nordemann, Handkommentar zum UWG, Einl., Rn. 134.

478 Götting in: Götting/Nordemann, Handkommentar zum UWG, Einl., Rn. 130.

479 Vgl. Fn. 475, Leitsatz 1.

480 Vgl. Fn. 475, Leitsatz 1.

481 Vgl. Fn. 478, Rn. 136.
} 
somit nicht für die nach dem UWG wesentlichen Bereiche der irreführenden und der vergleichenden Werbung. 482

Eine solche Stärkung des Marktortprinzips entspricht auch dem Sinn und Zweck der UGP-Richtlinie, da das Abstellen auf den Ort der Beeinträchtigung den Verbraucher und das Abstellen auf den Handlungsort den Unternehmer bevorteilt. ${ }^{483}$ Denn die Verbraucher sollen sich, soweit sie in ihrem Heimatland angesprochen werden, darauf verlassen können, dass die ihnen vertraute Rechtsordnung Anwendung findet. Darüber hinaus bestehen auch ganz grundsätzliche Argumente gegen das Konzept des Herkunftslandprinzips. So birgt dieses die Gefahr eines Wettkampfes unter den Staaten über das am wenigsten strenge Lauterkeitsrecht, um dadurch gegenüber anderen Staaten einen Wettbewerbsvorteil zu bieten. Dies steht jedoch der Verbesserung des Verbraucherschutzes als Zweck der UGP-Richtlinie ${ }^{484}$ diametral gegenüber. Denn ein solches Race-to-the-Bottom könnte dann nur dadurch verhindert werden, dass ein weltweit einheitliches Lauterkeitsrecht geschaffen würde. Um dies in jedem Fall mittelfristig auszuschließen bedarf es jedoch keiner Seherqualitäten. Denn selbst in Europa gibt es trotz der mit der UGP-Richtlinie angestrebten Vollharmonisierung noch kein einheitliches Lauterkeitsrecht, insbesondere da diese nur das Verhältnis von Unternehmern gegenüber Verbrauchern betrifft. ${ }^{485}$

\section{Persönliche Anwendbarkeit}

Das UWG schützt ausweislich seines $\ 1$ neben Mitbewerbern und Verbrauchern auch die sonstigen Marktteilnehmer sowie das Interesse der Allgemeinheit an der Erhaltung eines unverfälschten Wettbewerbes. Nicht umfasst ist dahingegen der Schutz sonstiger Allgemeininteressen, da dies nicht Aufgabe des Lauterkeitsrechts ist. ${ }^{486}$ Diese umfassende persönliche Anwendbarkeit wird als Schutzzwecktrias bezeichnet und wurde mit dem UWG 2004 gesetzlich verankert. Zwar war dies bereits zuvor durch Rechtsprechung anerkannt, formal schützte das UWG jedoch bis dato nur die einzelnen Mitbewerber. ${ }^{487}$ Das UWG weicht jedoch innerhalb bestimmter Regelung vom Grundsatz der einheitlichen Anwendung des Gesetzes gegenüber Mitbewerbern, Verbrauchern und sonstigen Marktteilnehmern ab, indem es die Anwendbarkeit bestimmter Normen auf Verbraucher beschränkt.

\footnotetext{
482 Köhler in: Köhler/Bornkamm, UWG, Einl., Rn. 5.42.

483 Art. 1 UGP-Richtlinie: „Zweck dieser Richtlinie ist es $\{. .$.$\} zum Erreichen eines bohen Verbraucherschutzes$ beizutragen."

484 Vgl. Fn. 483.

485 Vgl. Kapitel 2B.I.1.a.

486 Begründung der Bundesregierung für den Gesetzentwurf des UWG vom 22.08.2003, BT.Drs. 15/1487, S. 16.

487 Regierungsentwurf und Begründung zum UWG vom 22.08.2004, BT.-Drs. 15/1487, S. 15.
} 
Hierbei handelt es sich um die Regelungen aus den $\iiint_{3}$ Abs. $3^{488}$ und 5a Abs. 2 bis $4 \mathrm{UWG}^{489}$ sowie die sogenannte Verbrauchergeneralklausel aus $\int 3$ Abs. 2 UWG. ${ }^{490}$ Der Gesetzgeber hat diese Abweichungen damit begründet, den Geschäftsverkehr nicht mit den strengen Regelungen der UGP-Richtlinie belasten zu wollen, die für Verbraucher zwingend umzusetzen waren. 491

488 Vgl. Kapitel 5A.I.

${ }^{489}$ Vgl. Kapitel 6C.II.

490 Vgl. 0.

${ }^{491}$ Regierungsentwurf und Begründung zum UWG vom 20.08.2008, BT.-Drs. 16/10145, S. 22, 25. 



\section{Kapitel 5}

\section{Spezialregelungen zu geschäftlichen Handlungen im Zusammenhang mit Verhaltenskodizes}

Das UWG enthält drei Spezialregelungen zu geschäftlichen Handlungen im Zusammenhang mit Verhaltenskodizes. Diese Spezialregelungen wurden — wie die Definition des Verhaltenskodexes ${ }^{492}$ — mit dem UWG 2008 aufgenommen, um die Vorgaben der UGP-Richtlinie zu Verhaltenskodizes umzusetzen. 493 Sie betreffen somit die im Rahmen von geschäftlichen Handlungen getätigten unwahren Angaben, zu den Unterzeichnern eines Verhaltenskodexes zu gehören ${ }^{494}$ beziehungsweise über die Billigung eines Verhaltenskodexes durch eine öffentliche oder eine andere Stelle ${ }^{495}$ sowie Verstöße gegen die Verpflichtungen aus einem Verhaltenskodex. ${ }^{496}$

\footnotetext{
492 Vgl. Kapitel 3.

${ }^{493}$ Vgl. Kapitel 2B.I.2.

$494 \int 3$ Abs. 3 i. V. m. Nr. 1 Anhang zu $\int 3$ Abs. 3 UWG.

$495 \$ 3$ Abs. 3 i. V. m. Nr. 3 Anhang zu $\$ 3$ Abs. 3 UWG.

$496 \int 5$ Abs. 1 Nr. 6 UWG.
} 


\section{A. Geschäftliche Handlungen im Zusammenhang mit Verhaltenskodizes im Anhang zu $\ 3$ Abs. 3 UWG}

\section{Die Grundregelung in $₫ 3$ Abs. 3 UWG}

In Umsetzung des bereits dargestellten ${ }^{497}$ Art. 5 Abs. 5 der UGP-Richtlinie erklärt \ 3 Abs. 3 UWG alle im Anhang zum UWG aufgeführten geschäftlichen Handlungen für stets unzulässig, soweit diese sich gegen Verbraucher richten. Entgegen der missverständlichen Formulierung des $₫ 3$ Abs. 3 UWG sowie des Einleitungssatzes des Anhangs sind die im Anhang genannten Verhaltensweisen nicht per se als geschäftliche Handlungen einzuordnen, sondern nur dann, wenn die Voraussetzungen der geschäftlichen Handlung aus $₫ 2$ Abs. 1 Nr. 1 UWG ebenfalls vorliegen. ${ }^{498}$ Mit der Einordnung dieser geschäftlichen Handlungen als stets unzulässig weicht das UWG von seinem in der Generalklausel des $₫ 3$ Abs. 1 UWG enthaltenen Grundsatz ab, dass eine geschäftliche Handlung nur dann unzulässig ist, wenn sie die Interessen von Mitbewerbern, Verbrauchern oder sonstigen Marktteilnehmern ${ }^{499}$ spürbar beeinträchtigt. Diese sogenannte Relevanz- oder Bagatellklausel wurde mit der Novellierung des UWG 2004 als Teil der Generalklausel des $₫ 3$ UWG eingeführt, um unlautere Verhaltensweisen aus der Verfolgung durch das UWG herauszunehmen, die in der Praxis keine oder nur sehr geringe Auswirkungen auf die durch das UWG geschützten Rechtsgüter hatten. ${ }^{500}$ Mit dem UWG 2008 wurde sie zwar inhaltlich angepasst ${ }^{501}$, jedoch im Wesentlichen beibehalten. ${ }^{502}$ Für die von $\int 3$ Abs. 3 UWG erfassten geschäftlichen Handlungen gilt somit ein per-se-Verbot ohne Relevanzprüfung, so dass die vom Anhang zu $₫ 3$ Abs. 3 UWG erfassten geschäftlichen Handlungen vielfach als „Verbote obne Wertungsvorbebalt" bezeichnet werden. ${ }^{503}$ Entgegen dem Willen des deutschen Gesetz-

\footnotetext{
${ }^{497}$ Vgl. Kapitel 2B.I.2.

498 Schöttle, WRP 2009, 673, 674.

${ }^{499}$ Die sogenannte Schutzzwecktrias, vgl. Kapitel 4C.

500 Begründung der Bundesregierung für den Gesetzentwurf eines Gesetzes gegen den unlauteren Wettbewerb vom 22.08.2003, BT.-Drs. 15/1487, S. 16 f.

${ }^{501}$ Die Bagatellklausel des UWG 2008 stellt auch die Geeignetheit der geschäftlichen Handlung ab, „die Interessen von Mitbewerbern, Verbrauchern oder sonstigen Marktteilnehmern spürbar zu beeinträcbtigen." Dabei wurde das Merkmal der "Spürbarkeit" aus der Definition der „wesentlichen Beeinflussung des wirtschaftlichen Verbaltens des Verbrauchers "in Art. 2 lit. e) UGP-Richtlinie übernommen (Begründung der Bundesregierung für den Gesetzentwurf zur Änderung des UWG vom 20.08.2008, BT-Drs. 16/10145, S. 22). Die Bagatellklausel des UWG 2004 stellte dahingegen noch auf das — oftmals als unklar bezeichnete - Merkmal der ,nicht nur unerbeblichen Beeinträchtigung des Wettbewerbs zum Nacbteil von Marktteilnehmern" ab.

${ }^{502}$ Die Bagatellklausel ist weiterhin Bestandteil der Generalklausel des UWG, welche sich seit 2008 in $₫ 3$ Abs. 1 findet.

503 So unter anderem die Begründung zum Regierungsentwurf des UWG 2008, BT-Drs. 16/10145, S. 30 .
} 
gebers kann von diesem Automatismus auch in Sonderkonstellationen nicht aufgrund des Grundsatzes der Verhältnismäßigkeit abgewichen werden ${ }^{504}$, da dies den Vorgaben der UGP-Richtlinie widersprechen würde. ${ }^{505}$ Denn die UGPRichtlinie hat sich zur Schaffung größtmöglicher Rechtssicherheit gerade entschlossen, diese Verhaltensweisen ,unter allen Umständen“ als unlauter einzuordnen. ${ }^{506} \mathrm{Da}$ die UGP-Richtlinie hiervon keine Ausnahmen zulässt, widerspricht der Wille des deutschen Gesetzgebers der richtlinienkonformen Auslegung und hat somit zurückzutreten. Dies steht auch nicht im Widerspruch zum allgemeinen Verhältnismäßigkeitsprinzip. Denn dieses verhindert nicht das bedingungslose Verbot bestimmter Verhaltensweisen, sondern verpflichtet den diese Verbote aufstellenden Gesetzgeber lediglich dazu, dabei zwischen dem Ziel des Verbotes und den damit verbundenen Beeinträchtigungen abzuwägen. Genau dies haben die Richtliniengeber jedoch mit ihrer Entscheidung für eine größere Rechtssicherheit in diesen Sachverhalten getan, während sie für andere potenziell unlautere Verhaltensweisen zusätzliche Voraussetzungen (Täuschungsmoment und Relevanz für geschäftliche Entscheidung, Belästigung, Nötigung, etc.) 507 aufgenommen haben. Eine solche generelle Anwendung des Verhältnismäßigkeitsprinzips auf alle geschäftlichen Handlungen kann dabei auch nicht aus Erwägungsgrund 22 der UGP-Richtlinie abgeleitet werden. Denn dieser betrifft nicht die Einordnung von geschäftlichen Handlungen als unlauter beziehungsweise unzulässig, sondern die Sanktionierung derselben. ${ }^{508}$ Diese enge Auslegung darf jedoch nicht dazu verleiten, grundsätzlich auf eine Würdigung der Umstände eines jeden zu beurteilenden Einzelfalles zu verzichten. ${ }^{509}$ Denn selbstverständlich bedarf es dieser zur Feststellung, ob die Tatbestände des Anhangs zu \ 3 Abs. 3 UWG erfüllt sind.

\section{Der Anhang zu $\int 3$ Abs. 3 UWG}

Der deutsche Gesetzgeber hat im Rahmen der Umsetzung der UGP-Richtlinie in das UWG nicht nur das System einer Grundregelung zur Bestimmung der Rechtsfolge und der Aufführung der betroffenen Handlungen in einem Anhang übernommen, sondern im Wesentlichen auch den Anhang I zur UGP-Richtlinie. Lediglich die Nummer 26 des Anhangs I der UGP-Richtlinie wurde nicht in den Anhang zu $\int 3$ Abs. 3 UWG aufgenommen, sondern in $\int 7$ Abs. 2 UWG umgesetzt. Hierfür wurde, soweit die in Nr. 26 des Anhangs I der UGP-Richtlinie angeführten Geschäftspraktiken nicht bereits von den Nr. 2 und 3 des $\int 7$

\footnotetext{
504 Vgl. zum Willen des deutschen Gesetzgebers die Begründung zum Regierungsentwurf des UWG 2008, BT-Drs. 16/10145, S. 30.

505 Zweifelnd auch Sosnitza, WRP 2008, 1014, $1020 \mathrm{f}$.

506 Erwägungsgrund 17 der UGP-Richtlinie, Art. 5 Abs. 5 der UGP-Richtlinie.

507 Vgl. Artt. 6, 7, 8 und 9 UGP-Richtlinie.

508 A. A. Schöttle, WRP 2009, 673, 674.

509 Köhler in: Köhler/Bornkamm, Kommentar zum UWG, § 3 Rn. 25.
} 
Abs. 2 UWG umfasst waren, eine Anpassung des $\int 7$ Abs. 2 Nr. 1 UWG vorgenommen. 510 Zudem wurde die Regelung über den Umgang mit Gewinnzusagen, welche im Anhang I der UGP-Richtlinie in der Nummer 31 und damit bei den „aggressiven Geschäftspraktiken“" geregelt ist, im Anhang zum UWG unter der Nummer 17 übernommen, da der deutsche Gesetzgeber sie den ,irreführenden geschäftlichen Handlungen "zuordnete. 511 Darüber hinaus wurde auf die Unterteilung in irreführende und aggressive Geschäftspraktiken verzichtet sowie einige Formulierungen und Begrifflichkeiten (z. B. Unternehmer statt Gewerbetreibender ${ }^{512}$ ) geändert. Durch diese Änderungen sollten jedoch keine inhaltlichen Abweichungen bewirkt werden, sondern lediglich die Formulierungen mit der Terminologie des deutschen Lauterkeitsrechts im Besonderen und der deutschen Sprache im Allgemeinen in Einklang gebracht werden. ${ }^{513}$ Darüber hinausgehenden Änderungen standen dem deutsche Gesetzgeber auch nicht zu, da die UGP-Richtlinie den Katalog ihres Anhangs I als für alle Mitgliedstaaten verbindlich vorgab und festgelegte, dass dieser nur durch die Änderung der UGP-Richtlinie selbst geändert werden dürfe. ${ }^{514}$ Aufgrund ihres Ursprungs aus der UGP-Richtlinie ist damit bei der Auslegung der geschäftlichen Handlungen aus dem Anhang zu \ 3 Abs. 3 UWG das Gebot der richtlinienkonformen Auslegung zu beachten, welches auch der verfassungskonformen Auslegung vorzugehen hat. ${ }^{515}$ Dabei kann keine generelle Aussage dazu getroffen werden, ob die Tatbestände des Anhangs restriktiv oder weit auszulegen sind. ${ }^{516}$ Dies muss vielmehr für jeden Tatbestand einzeln unter Beachtung seiner Systematik und seines Regelungszweckes entschieden werden ${ }^{517}$, wobei die Tendenz aufgrund der strengen Rechtsfolge und der abschließenden Auflistung zur engen Auslegung gehen sollte. ${ }^{518}$ Darüber hinaus sind die Tatbestände des Anhangs zu \ 3 Abs. 3 UWG nicht analogiefähig. ${ }^{519}$

\section{Unwabre Angabe zu den Unterzeichnern eines Verbaltenskodexes zu gebören, Nr. 1 des Anhangs zu 3 Abs. $3 U W G$}

Der Anhang zu \3 Abs. 3 UWG erklärt in seiner Nr. 1 die unwahre Angabe eines Unternehmers, zu den Unterzeichnern eines Verhaltenskodexes i. S. d. \ 2 Abs. 1

\footnotetext{
510 Begründung der Bundesregierung für den Gesetzentwurf zur Änderung des UWG vom 20.08.2008, BT.-Drs. 16/10145, S. 16.

511 Vgl. Fn. 510, S. 33.

512 Vgl. zum Hintergrund Kapitel 3B

513 Vgl. Fn. 510, S. 30.

514 Art. 5 Abs. 5 S. 2 UGP-Richtlinie.

515 Köhler in: Köhler/Bornkamm, Kommentar zum UWG, \ 3, Rn. 26.

516 Sosnitza in: Piper/Ohly/Sosnitza, UWG, Anhang zu \ 3 Abs. 3, Rn. 4, a. A Beater, Unlauterer Wettbewerb, Rn. 1226.

517 Sosnitza in: Piper/Ohly/Sosnitza, UWG, Anhang zu \ 3 Abs. 3, Rn. 4.

518 Sosnitza, WRP 2008, 1014, 1021.

519 Beater, Rn. 1227.
} 
Nr. 5 UWG zu gehören, als unzulässig. Eine Angabe i. S. d. Norm ist dabei jede Aussage ${ }^{520}$, die sich auf Tatsachen bezieht und daher inhaltlich nachprüfbar ist. ${ }^{521}$ Dies entspricht auch den Anforderungen an den in der UGP-Richtlinie verwendeten Begriff der Behauptung 522 , der mit dem Begriff der Angabe umgesetzt wurde, und somit der richtlinienkonformen Auslegung. Keine Angaben sind dahingegen Meinungsäußerungen, deren Wahrheitsgehalt einer objektiven Nachprüfung nicht zugänglich ist. ${ }^{523}$ Jedoch ist die Grenze zwischen Meinungsäußerung und Tatsachenbehauptung oftmals fließend, so dass darauf abgestellt werden muss, ob der Meinungsäußerung ein nachprüfbarer Tatsachenkern zugrunde liegt. Liegt ein solcher nachprüfbarer Tatsachenkern vor, ist dieser die Angabe i. S. d. Nr. 1 des Anhangs zu \3 Abs. 3 UWG. ${ }^{524}$ Entsprechend kann eine Meinungsäußerung als Angabe anzusehen sein, soweit sie eine auf die Richtigkeit ihres Inhaltes nachprüfbare, dem Beweis zugängliche Aussage enthält. ${ }^{525}$ Die Angabe selbst kann dabei sowohl schriftlich als auch mündlich und sogar durch konkludente Handlungen oder Bilder erfolgen. ${ }^{526}$ Dies entspricht sowohl dem Zweck der UGPRichtlinie, zum Erreichen eines hohen Verbraucherschutzniveaus beizutragen ${ }^{527}$, als auch dem Willen des deutschen Gesetzgebers. Für Letzteren ergibt sich dies aus $\int 5$ Abs. 3 UWG, auch wenn sich die Konkretisierung an dieser Stelle auf Angaben i. S. d. $\int 5$ Abs. 1 S. 2 UWG bezieht. Dahingegen übersteigert die Ansicht, die UGP-Richtlinie verstehe unter einer Behauptung nur eine ausdrückliche Äußerung ${ }^{528}$, die Bedeutung des Wortlautes der UGP-Richtlinie. Diese ist in vielerlei Punkten, auch innerhalb ihres Anhang I, nicht in dem Maße konsistent ${ }^{529}$, dass allein aus der jeweiligen Auswahl der Begrifflichkeit der „Behauptung“ gegenüber der Begrifflichkeit des „Erweckens eines fälschlichen Eindruckes“ eine so weitgehende Folgerung statthaft wäre. ${ }^{530}$ Dies insbesondere, da in einigen Nummern des Anhang I der UGP-Richtlinie beide Begrifflichkeiten nebeneinander verwendet werden. 531

\footnotetext{
520 Wortbedeutung 1. nach http://www.duden.de/rechtschreibung/Angabe.

${ }^{521}$ Köhler in: Köhler/Bornkamm, UWG, \5, Rn. 2.37.

522 Eine Behauptung ist ihrer Wortbedeutung nach eine Äußerung, in der etwas als Tatsache hingestellt wird, Wortbedeutung 1a. nach http://www.duden.de/rechtschreibung/Behauptung .

523 Sosnitza in: Piper/Ohly/Sosnitza, UWG, $\int 5$, Rn. 89.

524 Sosnitza in: Piper/Ohly/Sosnitza, UWG, $\ 5$, Rn. 90.

525 Köhler in: Köhler/Bornkamm, UWG, \5, Rn. 2.39.

526 Büllesbach, S. 42 unter Anführung von Beispielen.

527 Art. 1 UGP-Richtlinie.

528 Bornkamm in: Köhler/Bornkamm, UWG, Anhang (zu \ 3 Abs. 3), Rn. 1.4.

${ }^{529}$ Der Anhang der UGP-Richtlinie verwendet unter anderem auch die Begrifflichkeiten „Behauptung, $\{\ldots\}$ soweit dies nicht der Fall ist" (Nr. 1, 3 und 4), ,falsche Behauptung" (Nr. 7 und 17.) sowie ,sachlich falsche Behauptung" (Nr. 12), ohne das hierdurch ein inhaltlicher Unterschied bezweckt wird.

530 Sosnitza in: Piper/Ohly/Sosnitza, UWG, Anhang zu $₫ 3$ Abs. 3, Rn. 5.

531 Nr. 9 und Nr. 22 des Anhangs I der UGP-Richtlinie.
} 
Von Nr. 1 des Anhangs zu $\ 3$ Abs. 3 UWG wird jede Angabe erfasst, mit welcher ein Unternehmer zu verstehen gibt, dass er sich auf einen Verhaltenskodex verpflichtet hat. Es reicht somit aus, wenn ein Unternehmer beispielsweise die Aussage trifft, für ihn gelte der Verhaltenskodex XY. Ein konkreter Hinweis auf die Unterzeichnung ist nicht notwendig. Nicht umfasst ist dahingegen die Aussage, sich an den Verhaltenskodex XY zu halten beziehungsweise dessen Vorgaben einzuhalten. Denn hieraus lässt sich nicht entnehmen, dass der Unternehmer den Verhaltenskodex tatsächlich abgegeben hat, sondern nur, dass er sich an dessen Vorgaben orientiert. Ebenfalls nicht erfasst von Nr. 1 des Anhangs zu $\ 3$ Abs. 3 UWG werden zudem Angaben über die Verpflichtungen durch den Verhaltenskodex oder dessen Inhalt im Allgemeinen. ${ }^{532}$

Unwahr und damit unzulässig sind diese von der Nr. 1 des Anhangs zu $\ 3$ Abs. 3 UWG umfassten Angaben dann, wenn der sie tätigende Unternehmer objektiv nicht zu den Unterzeichnern des Verhaltskodexes gehört. ${ }^{533}$ Auch diesbezüglich ist der Begriff der Unterzeichnung weit auszulegen. So ist kein formeller schriftlicher Unterzeichnungsakt notwendig, sondern es reicht jede tatsächliche Verpflichtung auf einen Verhaltenskodex aus. ${ }^{534}$ Denn auch wenn dies in der Praxis nur in seltenen Einzelfällen vorkommen dürfte, ist grundsätzlich auch eine mündliche Verpflichtung denkbar. ${ }^{535}$ Nicht notwendig ist eine darüber hinausgehende Behauptung des Unternehmers, die Verpflichtungen aus dem Verhaltenskodex würden eingehalten. ${ }^{536}$ Denn dies erwartet der Geschäftsverkehr nach Ansicht des Gesetzgebers bereits aufgrund der bloßen Bezugnahme auf die Unterzeichnereigenschaft. ${ }^{537}$ Entsprechend kann sich der Unternehmer auch nicht damit exkulpieren, dass er alle Verpflichtungen des angeblich unterzeichneten Verhaltenskodexes eingehalten hat.

Eindeutig unwahr ist eine solche Angabe, wenn der Unternehmer den Verhaltenskodex nie unterzeichnet hat oder der Verhaltenskodex überhaupt nicht existiert. Dabei ist allein entscheidend, ob der Unternehmer zu den Unterzeichnern eines Verhaltenskodexes gehört. Dabei ist es bei erwiesener Unwahrheit der Angabe unerheblich ist, ob der für die Angabe Verantwortliche sich der Unwahrheit der Angabe bewusst war beziehungsweise unter Beachtung der Erfordernisse

532 Dreyer, WRP 2007, 1294, 1299.

533 Sosnitza in: Piper/Ohly/Sosnitza, UWG, Anhang zu \ 3 Abs. 3, Rn. 6.

${ }_{534}$ Dreyer, WRP 2007, 1294, 1298.

535 Büllesbach, S. 42 f.

536 A. A. Berlit, II., Rn. 5, der die Behauptung der Einhaltung des Verhaltenskodexes voraussetzt, jedoch direkt im Anschluss feststellt, dass der Unternehmer nicht ausdrücklich behaupten muss, dass er die im Verhaltenskodex verankerten Standards einhält.

537 Begründung der Bundesregierung für den Gesetzentwurf zur Änderung des UWG vom 20.08.2008, BT.-Drs. 16/10145, S. 31. 
seiner beruflichen Sorgfalt eventuell sogar davon ausgehen durfte, dass die Angabe wahr ist. 538

Für diese Absolutheit spricht insbesondere die Ablehnung eines Vorschlages des Europäischen Parlamentes ${ }^{539}$ durch die Richtliniengeber, als zusätzliches Tatbestandsmerkmal die Bösgläubigkeit des Unternehmers vorauszusetzen. Diese strenge Regelung ist aufgrund der Intensität der damit einhergehenden Rechtsfolgen auch nicht unangemessen, da nach dem UWG nur die Unterlassung verschuldensunabhängig geschuldet wird. Die weitaus schärferen Schwerter (Schadensersatz und Gewinnabschöpfung) setzten dahingegen ein Verschulden des Unternehmers voraus. ${ }^{540}$

Anders sind dahingegen die Sachverhaltsvarianten zu bewerten, bei denen ein von einem Unternehmer unterzeichneter Verhaltenskodex — aus welchen Gründen auch immer — unwirksam ist oder wird beziehungsweise die Unterzeichnung selbst beispielsweise aufgrund mangelnder Vertretungsmacht des Unterzeichnenden nicht für das Unternehmen wirksam wird. In diesen Fällen ist mit Hinblick auf den Wortlaut ${ }^{541}$ eine formale Auslegung dergestalt vorzunehmen, dass es allein auf die Unterzeichnung des Verhaltenskodexes beziehungsweise die Verpflichtung auf den Verhaltenskodex ankommt. ${ }^{542}$ Hierfür spricht neben dem Wortlaut und der Vorgabe der engen Auslegung desselben die Praktikabilität der Rechtsanwendung. Denn anderenfalls wären die angerufenen Gerichte aufgefordert, in entsprechenden Verfahren inzident die Wirksamkeit des jeweiligen Verhaltenskodexes zu

538 Urteil d. EuGH v. 19.09.2013 (CHS Tour Services GmbH / Team4 Travel GmbH). Diesem Urteil des EuGH lag die Angabe der Team4 Travel GmbH zugrunde, dass bestimmte Unterkünfte zu bestimmten Zeiträumen exklusiv bei ihr gebucht werden könnten. Tatsächlich hatten die Unterkünfte, entgegen einer anderslautenden wirksamen vertraglichen Verpflichtung gegenüber dert Team4 Travel GmbH, auch anderen Anbietern in diesen Zeiträumen Kontingente zur Verfügung gestellt. Somit war die Angabe der Team4 Travel GmbH objektiv unwahr. Da der Team4 Travel GmbH dies jedoch zum Zeitpunkt der Tätigung ihrer Angabe nicht bewusst war und sie unter Anwendung ihrer beruflichen Sorgfalt aufgrund der wirksamen vertraglichen Vereinbarung mit den Unterkünften auch von der Wahrheit ihrer Angabe ausgehen durfte, legte das mit der Entscheidung befasste österreichische Gericht dem EuGH die Frage vor, ob im Rahmen des Art. 6 der UGP-Richtlinie als ungeschriebenes Tatbestandsmerkmal auch ein Verstoß gegen die berufliche Sorgfalt entsprechend des Grundtatbestandes aus Art. 5 Abs. 2a) UGPRichtlinie vorliegen müsse. Der EuGH hat diesbezüglich klargestellt, dass es bei einer Irreführung gemäß Art. 6 UGP-Richtlinie allein auf die in diesem enthaltenen Tatbestandsmerkmale ankomme.

539 Standpunkt des Europäischen Parlaments vom 20. April 2004, Dokument P5_TC1$\operatorname{COD}(2003) 0134$.

540 \9 Satz 1 UWG (Schadensersatz) und $\int 10$ Abs. 1 UWG (Gewinnabschöpfung).

541 Der UGP-Richtlinie und des UWG.

${ }^{542}$ In dieser Sachverhaltsvariante kann eine entsprechende Angabe jedoch nach $\int 5$ Abs. 1 Satz 1 UWG i. V. m. \5 Abs. 1 Satz 2 UWG zur Unlauterkeit der jeweiligen geschäftlichen Handlung führen, vgl. hierzu nachfolgend Kapitel 6B.II. 
prüfen. ${ }^{543}$ Die damit einhergehende Rechtsunsicherheit steht aber dem von der UGP-Richtlinie verfolgten Ziel der Rechtssicherheit entgegen. ${ }^{544}$ Entgegen der Ansicht der Gegenmeinung widerspricht eine solche formale Auslegung zudem nicht dem Zweck der Regelung, auch wenn der Gegenansicht dahingehend zuzustimmen ist, dass es dem Verbraucher bei Verhaltenskodizes selbstverständlich weniger auf den Unterzeichnungsakt, als vielmehr auf die Verpflichtung durch den Verhaltenskodex ankommt. ${ }^{545}$ Es liegt jedoch in keinem Fall im Interesse der Verbraucher, dass ein Unternehmer, der sich auf einen Verhaltenskodex verpflichtet und diesen auch eingehalten hat, durch das UWG belangt wird, weil der Verhaltenskodex selbst unwirksam ist. Denn es geht zu weit, das zentrale Element der Norm in der fehlenden tatsächlichen Bindung an einen Verhaltenskodex zu sehen. ${ }^{546}$ Zentrales Element der Regelung ist nach deren Sinn und Zweck vielmehr der Schutz von Verbrauchern vor solchen Unternehmern, die sich als Trittbrettfahrer an bestehende Verhaltenskodizes anhängen, ohne sich tatsächlich auf diese verpflichtet zu haben. Auch das fehlende Element der Bösgläubigkeit im Gesetzestext kann nicht als Begründung für die Ansicht der Gegenseite dienen. ${ }^{547}$ Denn dieses hätte sich — wie vorstehend bereits dargestellt — auf die Bösgläubigkeit bezüglich der Unterzeichnung, nicht bezüglich der Wirksamkeit des Verhaltenskodexes bezogen.

2. Unwabre Angabe über die Billigung eines Verbaltenskodexes, Nr. 3 des Anbangs zu $\int 3 A b s .3 U W G$

Ebenfalls in jedem Falle unzulässig ist nach Nr. 3 des Anhangs zu $\int 3$ Abs. 3 UWG die unwahre Angabe ${ }^{548}$ im Rahmen einer geschäftlichen Handlung, dass ein Verhaltenskodex von einer öffentlichen oder einer anderen Stelle gebilligt wurde. ${ }^{549}$ Hintergrund dieser Norm beziehungsweise ihrer Ursprungsnorm in der UGP-Richtlinie ${ }^{550}$ ist, dass in denjenigen europäischen Mitgliedstaaten, in welchen die Selbstregulierung im Lauterkeitsrecht eine besondere Bedeutung hat ${ }^{551}$, oftmals die Möglichkeit besteht, einen Verhaltenskodex durch eine (öffentliche) Stelle prüfen oder anerkennen zu lassen. So können im Vereinigten Königreich Ver-

\footnotetext{
543 Sosnitza in: Piper/Ohly/Sosnitza, UWG, Anhang (zu $₫ 3$ Abs. 3), Rn. 6.

${ }^{544}$ Erwägungsgrund 17 der UGP-Richtlinie.

545 Dreyer, WRP 2007, 1294, 1299.

546 Vgl. Fn. 545.

547 So aber Dreyer, WRP 2007, 1294, 1299.

${ }^{548}$ Hinsichtlich der Auslegung des Begriffs der (unwahren) Angabe sind die Ausführungen zu Nr. 1 des Anhangs des UWG sinngemäß zu übertragen (vgl. Kapitel 5A.II.1). Dabei ist unerheblich, dass der Unternehmer - wie auch in der UGP-Richtlinie der Gewerbetreibende - nicht ausdrücklich als Urheber der Abgabe benannt wird.
}

${ }^{549} \mathrm{Nr} .3$ des Anhangs zu $₫ 3$ Abs. 3 UWG.

$550 \mathrm{Nr} .3$ des Anhangs I der UGP-Richtlinie.

551 Vgl. Kapitel 2B.I.2. 
haltenskodizes vom staatlichen Office of Fair Trading offiziell anerkannt werden und diese Anerkennung auch wieder entzogen werden, wenn die für sie notwendigen Voraussetzungen nicht mehr vorliegen. 552 Die (erfolgreiche) Durchführung eines solchen Verfahrens war dabei für die an dem Verhaltenskodex beteiligten Unternehmen sinnvoll, da mit der Anerkennung ein erheblicher Vertrauensvorschuss der Verbraucher in diese Verhaltenskodizes verbunden war. ${ }^{553}$ Aber auch in Deutschland gibt es ein solches Anerkennungsverfahren für Wettbewerbsregeln durch das Bundeskartellamt 554 , obwohl die Selbstregulierung hierzulande keine ausgeprägte Tradition hat. 555

Sinn und Zweck der Nr. 3 des Anhangs zu $\int 3$ Abs. 3 UWG ist es daher, dass durch eine solche Billigung geschaffene besondere Vertrauen der Verbraucher in entsprechende Verhaltenskodizes zu schützen. Dabei wird ausdrücklich nicht nur die Billigung durch öffentliche Stellen erfasst, sondern auch die Billigung durch andere Stellen. Hierdurch erkennt der Gesetzgeber an, dass nicht nur die Billigung durch eine öffentliche Stelle geeignet ist, ein besonderes Vertrauen in einen Verhaltenskodex zu erzeugen, sondern jede Billigung durch eine von den Verpflichteten beziehungsweise Beteiligten eines Verhaltenskodexes unabhängige Stelle.556 Außerhalb dieser Voraussetzung der Billigung durch einen von den Verpflichteten beziehungsweise Beteiligten eines Verhaltenskodexes unabhängigen Dritten verbietet sich jede einschränkende Auslegung, da sich für eine solche keinerlei Hinweis in der UGP-Richtlinie oder deren Erwägungsgründen findet. Aus diesem Grund ist auch die von Büllesbach vertretene Einschränkung abzulehnen, wonach als andere Stelle i. S. d. Nr. 3 des Anhangs zu $₫ 3$ Abs. 3 UWG nur solche Stellen anzuerkennen sind, „die einen im Verbältnis zu "öffentlichen Stellen" vergleichbaren Glaubwürdigkeitsstatus besitzen “" 557 Dies insbesondere auch aus Gründen der Rechtssicherheit, da die Feststellung einer mit öffentlichen Stellen vergleichbaren Glaubwürdigkeit ein in höchstem Maße subjektives Tatbestandsmerkmal darstellt, welches zudem dem ständigen Risiko des Glaubwürdigkeitsverlustes der jeweiligen Stelle aufgrund besonderer Vorkommnisse unterliegt. ${ }^{558}$

Hinsichtlich der Einordnung als öffentliche Stelle kann auf die Definition des Begriffs im BDSG zurückgegriffen werden. Denn sowohl das BDSG als auch das

\footnotetext{
552 Section 8 (2) Enterprise Act 2002, abrufbar unter http://www.legislation.gov.uk/ukpga/ $2002 / 40 /$ section/8.

553 Büllesbach, S. $52 \mathrm{f}$.

554 \24 Abs. 3 ff. GWB. Zum Begriff der Wettbewerbsregel i. S. d. \$24 GWB vgl. Kapitel 3F.III.

555 Vgl. Kapitel 2A.

556 Dreyer, WRP 2007, 1294, 1300.

557 Büllesbach, S. 52.

${ }^{558}$ Entsprechend vermeidet Büllesbach im Rahmen der Nennung von Beispielen für Stellen mit vergleichbarem Glaubwürdigkeitsstatus wie öffentliche Stelle eine Festlegung und vermutet dies lediglich für Industrie- und Handelskammern, Wirtschaftsverbände, Verbraucherverbände und Gewerkschaften, S. 53.
} 
UWG und die UGP-Richtlinie dienen dem Verbraucherschutz. ${ }^{559}$ In entsprechender Anwendung des $₫ 2$ BDSG sind demnach die folgenden Stellen als öffentliche Stellen i. S. d. Nr. 3 des Anhangs zu \3 Abs. 3 UWG anzusehen: Sämtliche Behörden, Organe der Rechtspflege und andere öffentlich-rechtlich organisierte Einrichtungen des Bundes, der Länder, der Gemeinden und Gemeindeverbände, der bundesunmittelbaren Körperschaften, Anstalten und Stiftungen des öffentlichen Rechts und deren Vereinigungen sowie alle sonstigen juristischen Personen des öffentlichen Rechts und deren Vereinigungen, soweit sie der Aufsicht eines Landes unterstehen, wobei die Rechtsform unbeachtlich ist. Selbst Vereinigungen privaten Rechts sind somit öffentliche Stellen ${ }^{560}$, wenn sie Aufgaben der Verwaltung wahrnehmen und vom Bund beherrscht werden. 561

Im Hinblick auf den Sinn und Zweck der Norm, das Vertrauen der Verbraucher in die Prüfung eines Verhaltenskodexes durch unabhängige Dritte zu schützen, muss der Begriff der Billigung i. S. d. Nr. 3 des Anhangs zu $\ 3$ Abs. 3 UWG zum bestmöglichen Schutz der Verbraucher jegliche Angabe umfassen, die in vergleichbarer Art und Weise vertrauensbildend wirkt. So beispielsweise die Angabe, dass ein Verhaltenskodex befürwortet, unterstützt, gutgeheißen, genehmigt oder anerkannt werde. Diese entspricht auch der Bedeutung des in der englischen Fassung der UGP-Richtlinie verwendeten Begriffs ,endorsement", welcher als Billigung, aber auch als Befürwortung, Anerkennung oder Unterstützung übersetzt werden kann. ${ }^{562}$ Entsprechend wird in der Literatur als Beispiel für eine Billigung durch eine öffentliche Stelle i.S.d. Norm die bereits angeführte Anerkennung einer Wettbewerbsregel durch das Bundeskartellamt genannt. ${ }^{563}$

Wie bei Nr. 1 des Anhangs zu $\ 3$ Abs. 3 UWG $^{564}$ ist für die Unzulässigkeit aufgrund von Nr. 3 des Anhangs zu $\ 3$ Abs. 3 UWG allein entscheidend, ob die Angabe objektiv unwahr ist, also tatsächlich keine Billigung des Verhaltenskodexes durch eine öffentliche oder eine andere Stelle besteht. ${ }^{655}$ Entsprechend ist es unerheblich, ob die Angabe im guten Glauben an eine Billigung erfolgt ist. ${ }^{566}$ Liegt tatsächlich keine entsprechende Billigung vor, ist die geschäftliche Handlung un-

\footnotetext{
${ }^{559}$ Das BDSG betrifft gemäß seines $\int 1$ den Schutz des Einzelnen vor Beeinträchtigungen in seinem Persönlichkeitsrecht, das UWG laut seines $\ 1$ den Schutz der Verbraucher und Verbraucherinnen vor unlauteren geschäftlichen Handlungen und UGP-Richtlinie hat gemäß ihres Art. 1 den Zweck, zur Erreichung eines hohen Verbraucherschutzniveaus beizutragen.

${ }^{560}$ Vgl. $\ 2$ Abs. 3 BDSG.

${ }^{561}$ Eine Beherrschung liegt vor, wenn dem Bund die absolute Mehrheit der Anteile gehört oder die absolute Mehrheit der Stimmen zusteht, vgl. \2 Abs. 3 Nr. 2 BDSG.

$562 \mathrm{http}: / /$ dict.leo.org/ende/index_de.html\#/search=endorsement\&searchLoc=0\&resultOrder= basic\&multiwordShowSingle $=$ on, zuletzt abgerufen am 20.03.2013.

563 Sosnitza in: Piper/Ohly/Sosnitza, UWG, Anhang (zu \3 Abs. 3), Rn. 9, Bornkamm in: Köhler/Bornkamm, UWG, Anhang zu $₫ 3$ Abs. 3, Rn. 3.1.

564 Vgl. Kapitel 5A.II.1.

565 Bornkamm in: Köhler/Bornkamm, UWG, Anhang zu \3 Abs. 3, Rn. 3.3.

566 Vgl. zur Unerheblichkeit der Bösgläubigkeit Kapitel 5A.II.1.
} 
zulässig. Auch ob sich die Angabe auf ein tatsächlich bestehendes Billigungssystem bezieht oder auf eine Billigung durch eine der genannten Stellen verweist, obwohl diese hierfür weder zuständig ist noch eine entsprechende Billigung ausspricht, ist nicht von Bedeutung. ${ }^{567}$ Denn diese Angaben wird der Verbraucher bereits der Angabe, der Verhaltenskodex sei von einer öffentlichen oder einer anderen Stelle gebilligt worden, entnehmen. ${ }^{568}$

Keine unwahre Angabe über eine bestehende Billigung liegt vor, wenn eine öffentliche oder eine andere Stelle einen Verhaltenskodex gebilligt hat, obwohl die Anforderungen, die sie selbst an eine Billigung stellt, nicht vorlagen. Diese Sachverhalte unterfallen nicht dem Anwendungsbereich der Nr. 3 des Anhangs zu $\int 3$ Abs. 3 UWG, da in diesem Fall ja tatsächlich eine Billigung erfolgt ist und somit die entsprechende Angabe wahr ist. Dabei ist es unerheblich, ob die ausgesprochene Billigung nicht hätte erfolgen dürfen.

\section{Zusammenfassung}

Sobald ein Unternehmer gegenüber einem Verbraucher wahrheitswidrig in irgendeiner Art und Weise angibt, sich auf einen Verhaltenskodex verpflichtet zu haben, ist die geschäftliche Handlung, innerhalb welcher der Unternehmer diese Angabe getätigt hat, nach \3 Abs. 3 UWG i. V.m. der Nr. 1 des Anhangs zu \ 3 Abs. 3 UWG ohne Ansehen der Relevanz dieser Angabe für den Verbraucher unzulässig. Zur Feststellung der Unwahrheit dieser Angabe kommt es allein darauf an, ob der Unternehmer sich tatsächlich auf diesen Verhaltenskodex verpflichtet hat. ${ }^{569}$ Ist dies der Fall, ist es sogar unerheblich, dass der Verhaltenskodex selbst unwirksam ist oder die Unterzeichnung selbst beispielsweise aufgrund fehlender Vertretungsmacht des Unterzeichnenden für den Unternehmer unwirksam war.

Gleichfalls in jedem Fall unzulässig ist nach $\int 3$ Abs. 3 UWG i. V. m. der Nr. 3 des Anhangs zu \3 Abs. 3 UWG die im Rahmen einer geschäftlichen Handlung gegenüber einem Verbraucher getätigte unwahre Angabe, ein Verhaltenskodex sei von einer öffentlichen oder einer anderen Stelle gebilligt worden. Dabei muss nicht der Begriff der Billigung verwendet werden, sondern es reicht jede Angabe aus, welche bei einem Verbraucher ein vergleichbares Vertrauen in die Qualität des Verhaltenskodexes schafft. Für die Feststellung, ob eine Stelle eine öffentliche Stelle i. S. d. Nr. 3 des Anhangs zu \ 3 Abs. 3 UWG darstellt, kann auf $\int 2$ BDSG zurückgegriffen werden. Eine andere Stelle i.S. d. Nr. 3 des Anhangs zu \ 3 Abs. 3 UWG ist jede von den Verpflichteten beziehungsweise den Beteiligten des

\footnotetext{
567 Büllesbach, S. 52, Dreyer, WRP 2007, 1294, 1300.

568 Dreyer, WRP 2007, 1294, 1300.

569 Sosnitza in: Piper/Ohly/Sosnitza, UWG, \5, Rn. 90.
} 
Verhaltenskodexes unabhängige Stelle ohne Ansehen von deren Glaubwürdigkeitsstatus. ${ }^{570}$ Auch bei dieser Angabe ist allein entscheidend, ob tatsächlich eine entsprechende Billigung besteht oder nicht. ${ }^{571}$

\section{B. Irreführende Angabe über die Einhaltung eines Verhaltenskodex, $\ 5$ Abs. 1 Satz 2 Nr. 6 UWG}

Eine weitere Spezialregelung zu geschäftlichen Handlungen im Zusammenhang mit Verhaltenskodizes enthält die $\ 5$ Abs. 1 Satz 2 UWG. ${ }^{572}$ Dessen Nr. 6 bestimmt, unter welchen Voraussetzungen der Verstoß gegen Verpflichtungen aus Verhaltenskodizes eine irreführende geschäftliche Handlung darstellt. Auch diese Regelung hat ihren Ursprung in der UGP-Richtlinie ${ }^{573}$ und ist daher richtlinienkonform auszulegen.

I. Systematik der Norm, Prüfungszusammenhang mit $₫ 3$ Abs. 1 UWG

\5 Abs. 1 UWG bestimmt in seinem Satz 1 entsprechend der Systematik der UGP-Richtlinie ${ }^{574}$ die Vornahme irreführender geschäftlicher Handlungen als unlauter, während sein Satz 2 definiert, wann eine solche irreführende geschäftliche Handlung vorliegt. In Abweichung zur Systematik der UGP-Richtlinie ${ }^{575}$ führt die Unlauterkeit einer geschäftlichen Handlungen im UWG jedoch nicht automatisch zur Unzulässigkeit ${ }^{576}$ beziehungsweise zum Verbot ${ }^{577}$ der geschäftlichen Handlung. Hierfür ist nach $₫ 3$ Abs. 1 UWG zusätzlich erforderlich, dass unlautere geschäftliche Handlungen, geeignet sind, die Interessen von Mitbewerbern, Verbrauchern oder sonstigen Marktteilnehmern spürbar zu beeinträchtigen “. .578 Für die Annahme einer solchen spürbaren Beeinträchtigung kommt es nach dem Willen des Gesetzgebers maßgeblich darauf an, „ob die $2 u$ beurteilende geschäftliche Handlung geeignet ist, das wirtschaftliche Verbalten eines Durchschnittsverbrauchers wesentlich zu beeinflussen. "579 Zur Beantwortung der Frage, wann eine solche wesentliche Beeinflussung des wirtschaft-

\footnotetext{
570 Dreyer, WRP 2007, 1294, 1300.

571 Bornkamm in: Köhler/Bornkamm, UWG, Anhang zu \ 3 Abs. 3, Rn. 3.1.

$572 § 5$ UWG, vgl. hierzu nachfolgend auch Kapitel 6B.

573 Art. 6 Abs. 2 lit. b) UGP-Richtlinie, vgl. Kapitel 2B.I.2.

574 Art. 5 Abs. 4 lit. a) UGP-Richtlinie, vgl. auch Kapitel 2B.I.1.b.

575 Vgl. Kapitel 2B.I.1.b

576 So die Terminologie des UWG, $\ 3$ Abs. 1.

577 So die Terminologie der UGP-Richtlinie, Art. 5 Abs. 1.

578 Vgl. bereits die Ausführungen zur Relevanzklausel unter Kapitel 5A.I

579 Begründung der Bundesregierung für den Gesetzentwurf zur Änderung des UWG vom 20.08.2008, BT.-Drs. 16/10145, S. 16.
} 
lichen Verhaltens vorliegt, ist unter Rückgriff auf Art. 2 lit. e) UGP-Richtlinie 580 im Wesentlichen darauf abzustellen, ob durch die geschäftliche Handlung jemand zu einer geschäftlichen Entscheidung veranlasst wurde, die er andernfalls nicht getroffen hätte. Dieses Erfordernis der spürbaren Beeinträchtigung der Interessen durch die unlautere geschäftliche Handlung zur Annahme der Unzulässigkeit ist richtlinienkonform ${ }^{581}$, da die UGP-Richtlinie in ihrem Art. 6 Abs. 1 bereits für die Annahme einer Irreführung voraussetzt, dass die geschäftliche Handlung ,den Durchschnittsverbraucher $\{. .$.$\} in jedem Fall tatsächlich oder voraussichtlich zu einer geschäftli-$ chen Entscheidung veranlasst, die er ansonsten nicht getroffen hätte." Insofern stellt das Erfordernis der spürbaren Beeinträchtigung der Interessen durch die unlautere geschäftliche Handlung aus \3 Abs. 1 UWG zwar eine systematische, aber keine inhaltliche Unterscheidung zur UGP-Richtlinie dar. Denn das UWG verlagert damit das Tatbestandsmerkmal der Eignung zur Veranlassung zu einer geschäftlichen Entscheidung lediglich von der Irreführung ${ }^{582}$ auf die Unzulässigkeit. Dies bedeutet jedoch ${ }^{583}$, dass $\int 5$ UWG nicht isoliert von $\int 3$ Abs. 1 UWG angewendet und geprüft werden darf ${ }^{584}$ und kann. Denn allein die Tatsache, dass eine geschäftliche Handlung irreführend und damit unlauter ist, hat keine Rechtsfolgen nach dem UWG. Voraussetzung hierfür ist immer, dass die geschäftliche Handlung unzulässig ist. ${ }^{585}$

\section{Die Voraussetzungen der Irreführung aus $\int 5$ Abs. 1 Satz 2 UWG}

Voraussetzung einer irreführenden geschäftlichen Handlung ist nach \5 Abs. 1 Satz 2 UWG zunächst, dass diese eine unwahre Angabe ${ }^{586}$ oder eine sonstige zur Täuschung geeignete Angabe über die in den Nummern 1 bis 7 aufgeführten Umstände enthält. Dies entspricht den Voraussetzungen der Irreführung nach Art. 6 Abs. 1 UGP Richtlinie. ${ }^{587}$ In der UGP-Richtlinie gelten diese Voraussetzungen jedoch nur für die in Art. 6 Abs. 1 lit. a) bis g) UGP-Richtlinie genannten Umstände, welche in den Nummern 1 bis 5 und der Nummer 7 von $\int 5$ Abs. 1

\footnotetext{
580 Art. 2 lit. e) UGP-Richtlinie: „,,wesentliche Beeinflussung des wirtschaftlichen Verhaltens des Verbrauchers" die Anwendung einer Geschäftspraxis, um die Fähigkeit des Verbrauchers, eine informierte Entscheidung zu treffen, spürbar zu beeinträchtigen und damit den Verbraucher zu einer geschäftlichen Entscheidung zu veranlassen, die er andernfalls nicht getroffen bätte."

581 Begründung der Bundesregierung für den Gesetzentwurf zur Änderung des UWG vom 20.08.2008, BT.-Drs. 16/10145, S. 16.

582 So in der UGP-Richtlinie, Art. 6 Abs. 1.

583 Beater, Rn. 1288.

584 Ansonsten läge bei geschäftlichen Handlungen gegenüber Verbrauchern ein Verstoß gegen das Gebot der richtlinienkonformen Auslegung vor.

585 \ 3 Abs. 1 UWG.

586 Zum Begriff der Angabe vgl. Kapitel 5A.II.1.

587 Soweit sie durch $\int 5$ Abs. 1 Satz 2 UWG umgesetzt werden, vgl. Kapitel 5B.I.
} 
Satz 2 UWG umgesetzt wurden. ${ }^{588}$ Insoweit ist die Regelung im UWG somit eindeutig richtlinienkonform. Eine Sonderstellung in der Auflistung nimmt hingegen der geschäftliche Handlungen im Zusammenhang mit Verhaltenskodizes betreffende Umstand aus $\int 5$ Abs. 1 Nr. 6 UWG ein. Denn dieser dient der Umsetzung von Art. 6 Abs. 2 lit. b) UGP-Richtlinie ${ }^{589}$ und ist entsprechend - zumindest soweit geschäftliche Handlungen gegenüber Verbrauchern betroffen sind - an diesem zu messen.

Die im Einleitungssatz des Art. 6 Abs. 2 UGP-Richtlinie benannten Voraussetzungen unterscheiden sich jedoch von denen im Einleitungssatz des Art. 6 Abs. 1 UGP-Richtlinie und damit auch von denen des $\int 5$ Abs. 1 Satz 2 UWG. Denn der Einleitungssatz von Art. 6 Abs. 2 UGP-Richtlinie erfordert - insoweit in Übereinstimmung mit dem Einleitungssatz von Art. 6 Abs. 1 UGP-Richtlinie 590 - lediglich, dass die betroffene geschäftliche Handlung ,im konkreten Fall unter Berücksichtigung aller tatsächlichen Umstände einen Durchschnittsverbraucher zu einer geschäftlichen Entscheidung veranlasst oder zu veranlassen geeignet ist, die er ansonsten nicht getroffen bätte" und damit die Voraussetzung, welche im UWG im Rahmen des \3 Abs. 1 geprüft wird.591 Abweichend von dem Einleitungssatz des Art. 6 Abs. 1 UGPRichtlinie (und damit auch von $\int 5$ Abs. 1 Satz 2 UWG) setzt der Einleitungssatz von Art. 6 Abs. 1 UGP-Richtlinie dahingegen für die Irreführung nicht voraus, dass die geschäftliche Handlung, ,falsche Angaben enthält und somit unwahr ist oder $\{\ldots\}$ in irgendeiner Weise $\{\ldots\}$ den Durchschnittsverbraucher $\{\ldots\}$ täuscht oder ibn zu täuschen geeignet ist."

Nach Ansicht des deutschen Gesetzgebers stellt dieser Unterschied zwischen den beiden Einleitungssätzen der Absätze 1 und 2 des Art. 6 UGP-Richtlinie nur eine sprachliche, aber keine inhaltlich Abweichung dar. ${ }^{592}$ Dieser Ansicht muss unter alleiniger Betrachtung der Einleitungssätze widersprochen werden. Denn selbstverständlich stellt die Notwendigkeit einer unwahren oder sonstigen zur Täuschung geeigneten Angabe innerhalb der zu beurteilenden geschäftlichen Handlung als zusätzliche Voraussetzung auch einen inhaltlichen Unterschied dar. Für einen solchen inhaltlichen Unterschied spricht auch die Umsetzung des Art. 6 Abs. 2 lit. a) UGP-Richtlinie durch den deutschen Gesetzgeber in $\int 5$ Abs. 2 UWG. ${ }^{593}$ Denn würde zwischen den beiden Einleitungssätzen der Absät-

\footnotetext{
588 Begründung der Bundesregierung für den Gesetzentwurf zur Änderung des UWG vom 20.08.2008, BT.-Drs. 16/10145, S. 23.

589 Vgl. Fn. 588, S. 23 f.

590 Art. 6 Abs. 1 UGP-Richtlinie: „Eine Geschäftspraxis gilt als irrefübrend, wenn sie $\{\ldots\}$ den Durchschnittsverbraucher $\{. .$.$\} in jedem Fall tatsächlich oder voraussichtlich zu einer geschäftlichen Entscheidung veranlasst,$ die er ansonsten nicht getroffen bätte. "

591 Vgl. Kapitel 5B.I.

592 Vgl. Fn. 588.

593 Begründung der Bundesregierung für den Gesetzentwurf zur Änderung des UWG vom 20.08.2008, BT.-Drs. 16/10145, S. 16 f., S. 24.
} 
ze 1 und 2 des Art. 6 UGP-Richtlinie tatsächlich kein inhaltlicher Unterschied bestehen, hätte der deutsche Gesetzgeber Art. 6 Abs. 2 lit. a) UGP-Richtlinie statt in einem eigenen Absatz auch als Nr. 8 der Auflistung in $\int 5$ Abs. 1 Satz 2 UWG umgesetzten können. Tatsächlich aber hat der deutsche Gesetzgeber Art. 6 Abs. 2 lit. a) UGP-Richtlinie lediglich leicht sprachlich angepasst ${ }^{594}$ als $\int 5$ Abs. 2 UWG übernommen, so dass in diesen Sachverhalten auch nach dem UWG nur die Voraussetzungen des Einleitungssatzes des Art. 6 Abs. 2 UGP-Richtlinie für die Einordnung als irreführende Handlung vorliegen müssen.

Aber auch bei ganzheitlicher Betrachtung des Einleitungssatzes von Art. 6 Abs. 2 UGP-Richtlinie und seiner lit. b) verbleiben noch inhaltliche Unterschiede zum Einleitungssatz von $\int 5$ Abs. 1 Satz 2 UWG i. V. m. seiner Nr. 6. So mag die Nichteinhaltung von Verpflichtungen aus einem Verhaltenskodexes als zentrales Merkmal von lit. b) des Art. 6 Abs. 2 UGP-Richtlinie inhaltlich mit dem Unrechtsgehalt einer unwahren Angabe über die Einhaltung der Verpflichtungen aus einem Verhaltenskodex vergleichbar sein. Dies gilt jedoch nicht gleichermaßen für die zweite Alternative von $\int 5$ Abs. 1 Satz 2 UWG entsprechend des Einleitungssatzes von Art. 6 Abs. 1 UGP-Richtlinie. Denn nach dieser können auch sachlich richtige Angaben für die Annahme einer Irreführung ausreichen, wenn sie geeignet sind, ihren Empfänger zu täuschen. Eine sachlich richtige Angabe über die Einhaltung der Verpflichtungen aus einem Verhaltenskodex und somit schlichtweg die Einhaltung eines Verhaltenskodexes kann jedoch im Hinblick auf ihren Unrechtsgehalt in keinem Fall mit der Nichteinhaltung der Verpflichtungen aus einem Verhaltenskodex gleichgesetzt werden. Dies muss selbst dann gelten, wenn diese sachlich richtige Angabe in irgendeiner Art und Weise geeignet ist, jemanden $\mathrm{zu}$ täuschen. Aus diesem Grund ist es nicht mit der UGP-Richtlinie vereinbar, dass der deutsche Gesetzgeber bewusst auch wahre, aber zur Täuschung geeignete Angaben über die Einhaltung eines Verhaltenskodexes als irreführende geschäftliche Handlung eingestuft hat. ${ }^{595}$

Entsprechend ist $\int 5$ Abs. 1 Satz 2 UWG insoweit, als der Umstand aus seiner Nr. 6 betroffen ist, dahingehend einschränkend auszulegen, dass lediglich geprüft wird, ob ein Verstoß gegen einen Verhaltenskodex vorliegt. Liegt kein solcher Verstoß gegen einen Verhaltenskodex vor, scheidet eine Irreführung aufgrund von $\int 5$ Abs. 1 Satz 2 Nr. 6 UWG aus. Dies ergibt sich im Hinblick auf geschäftliche Handlungen gegenüber Verbrauchern aus dem Gebot der richtlinienkonformen Auslegung. Da der Gesetzgeber den Art. 6 Abs. 2 lit. b) UGP-Richtlinie

\footnotetext{
594 So wird wie im gesamten UWG (vgl. zur Begründung Fn. 426) der Begriff Produkt nicht verwendet, sondern entsprechend der Definition in Art. 2 lit. c) UGP-Richtlinie als Ware und Dienstleistung umschrieben.

595 Begründung der Bundesregierung für den Gesetzentwurf zur Änderung des UWG vom 20.08.2008, BT.-Drs. 16/10145, S. 24.
} 
richtlinienkonform umsetzen wollte ${ }^{596}$, gilt diese einschränkende Auslegung aber auch für geschäftliche Handlungen gegenüber Unternehmern und sonstigen Marktteilnehmern.

III. Die Voraussetzungen der Nr. 6 des $\ 5$ Abs. 1 Satz 2 UWG

Unter welchen Voraussetzungen der Verstoß gegen eine Verpflichtung aus einem Verhaltenskodex nach dem UWG irreführend ist, ergibt sich aus $₫ 5$ Abs. 1 Satz 2 Nr. 6 UWG. Nach diesem können unwahre Angaben 597 über ,die Einhaltung eines Verbaltenskodexes, auf den sich der Unternehmer verbindlich verpflichtet hat, wenn er auf diese Bindung binweist" eine Irreführung bedeuten. Obwohl die Formulierung in Wortlaut und Aufbau erheblich von der Ursprungsnorm in der UGP-Richtlinie ${ }^{598}$ abweicht, ist diese für die Auslegung des $₫ 5$ Abs. 1 Satz 2 Nr. 6 UWG maßgeblich. Dies ergibt sich sowohl aus dem Grundsatz der richtlinienkonformen Auslegung als auch dem vom deutschen Gesetzgeber verfolgten Sinn und Zweck der Regelung. Denn $\int 5$ Abs. 1 Satz 2 Nr. 6 UWG sollte bezüglich der Voraussetzungen mit Art. 6 Abs. 2 lit. b) UGP-Richtlinie übereinstimmen. ${ }^{599}$ Zudem zeigt der Vergleich der Regelungen, dass diese, trotz der sprachlichen Unterschiede, jeweils eine Beschränkung auf Verhaltenskodizes mit einem besonderen Grad der Verpflichtung ${ }^{600}$ enthalten und einen Hinweis auf die Bindung an den Verhaltenskodex erfordern.

\section{Verbindliche Verpflichtung auf Verbaltenskodex}

Im Hinblick auf den besonderen Grad der Verpflichtung durch den Verhaltenskodex fordert die Regelung des UWG, dass sich der Unternehmer „verbindlich verpflicbtet" hat. Entscheidend ist somit zunächst, dass sich der Unternehmer selbst auf den Verhaltenskodex verpflichtet hat. Verpflichtungen aus den Verhaltenskodizes Dritter muss er nicht einhalten. Die Ursprungsnorm in der UGPRichtlinie ${ }^{601}$ setzt diesbezüglich voraus, dass es sich bei dem Verhaltenskodex „nicht um eine Absichtserklärung, sondern um eine eindentige Verpflichtung handelt, deren Einhaltung nachprüfbar ist".602 Im Hinblick auf die richtlinienkonforme Auslegung definiert diese Sequenz aus der UGP-Richtlinie somit den im UWG verwendeten unbestimmten Rechtsbegriff der ,verbindlichen Verpflichtung".

\footnotetext{
596 Vgl. Fn. 593.

$597 \mathrm{Zu}$ den eingeschränkten Voraussetzungen des $₫ 5$ Abs. 1 Satz 2 UWG vgl. Kapitel 5B.II.

598 Art. 6 Abs. 2 lit. b) UGP-Richtlinie, zum Wortlaut der Norm vergleiche Kapitel 2B.I.2.b.

599 Begründung der Bundesregierung für den Gesetzentwurf zur Änderung des UWG vom 20.08.2008, BT.-Drs. 16/10145, S. 24.

${ }^{600} \mathrm{Vgl}$ Kapitel 3D.

601 Vgl. Art. 6 Abs. 2 lit. b) i) UGP-Richtlinie.

${ }^{602}$ Zur Frage des Bezugs der Einschränkung durch Art. 6 Abs. 2 lit. b) i) UGP-Richtlinie auf den betroffenen Verhaltenskodex vgl. die Ausführungen unter Kapitel 5A.II.
} 
Was die Richtliniengeber wiederum unter einer solchen „eindeutigen Verpflichtung, deren Einhaltung nachprüfbar ist" verstanden, erschließt sich aus den Materialien zur Richtlinie. So wollte die Generaldirektion Gesundheit und Verbraucher, welche den Entwurf der UGP-Richtlinie erstellt hatte, bereits nur echte Verpflichtungen als Täuschungen werten. ${ }^{603}$ Dahingegen sollte die Nichteinhaltung einer bloßen Verpflichtungsintention „nach dem Grundsatz des redlichen Bemühens" nicht ausreichen, um den Tatbestand der Irreführung zu erfüllen. ${ }^{604}$ Die hierfür gewählte Formulierung ${ }^{605}$ erschien dem Europäischen Parlament jedoch nicht eindeutig genug, so dass es in seinem Standpunkt zur UGP-Richtlinie forderte, im Rahmen der Begriffsbestimmungenen in Art. 2 UGP-Richtlinie den Begriff der ,festen Verpflichtung" als lit. 1a) aufzunehmen und wie folgt zu definieren: Eine feste Verpflichtung ist ,eine Verpflichtung in einem Verbaltenskodex, die die Unterzeichner bezüglich der Geschäftspraktiken, auf die sich die Richtlinie bezieht, speziell zu einer bestimmten Handlung oder zu Handlungsweisen gegenüber dem Verbraucher verpflichtet. Es sind jegliche Verpflichtungen ausgeschlossen, die im Kodex selbst speziell von der Klassifizierung als feste Verpflichtungen ausgeschlossen sind oder die nur als Ziele oder Bestrebungen bezeichnet werden. "006 Das Europäische Parlament begründete diese Forderung ,mit den Auswirkungen im Zusammenhang mit diesem Begriff" im Rahmen der Anwendung der UGP-Richtlinie auf Verhaltenskodizes und der damit verbundenen Notwendigkeit, „eine feste Verpflichtung deutlich von einer einfachen Bestrebung abzugrenzen". ${ }^{607} \mathrm{Da}$ der Begriff der festen $V$ erpflichtung im Entwurf der Kommission ${ }^{608}$ nicht verwendet wurde, bezog der Europäische Rat ${ }^{609}$ diese Änderungsanregung zu Recht auf den in Art. 6 Abs. 2 lit. b) des Richtlinienvorschlages der Europäischen Kommission ${ }^{610}$ verwendeten Begriff der eindeutigen Verpflichtung und fügte dort zur Verdeutlichung den Begriff der Absichtserklärung als negatives Tatbestandsmerkmal ein. ${ }^{611}$ Dabei beschränkt sich die Bedeutung des negativen Tatbestandsmerkmals der Absichtserklärung

${ }^{603}$ KOM (2002) 289 endgültig, S. 13, Tz. 29,

${ }^{604}$ Vgl. Fn. 603, vgl. hierzu auch die Ausführungen zum Begriff der Absichtserklärung unter Kapitel $3 \mathrm{D}$.

605 Art. 6 Abs. 2 lit. b) des Vorschlags der Europäischen Kommission für eine UGP-Richtlinie (KOM (2003) 356 endgültig) lautete: „,...) die Nichteinhaltung von Verpflichtungen, die der Gewerbetreibende im Rahmen von Verbaltenskodizes, auf die er sich verpflicbtet hat, eingegangen ist,

- sofern es sich um eine eindeutige Verpflichtung handelt, deren Einbaltung nachprïfbar ist (...)“. Er enthielt somit bereits das positive Tatbestandsmerkmal der heutigen Regelung, welches letztlich nur um die Klarstellung, dass Absichtserklärungen nicht ausreichen, erweitert wurde.

606 A5-0188/2004 endgültig, Entwurf einer legislativen Entschließung des Europäischen Parlaments, Änderungsantrag 24, S. 18.

607 Vgl. Fn. 606.

${ }^{608} \mathrm{KOM}(2003) 356$ endgültig.

${ }^{609} \mathrm{KOM}(2004) 753$ endgültig, S. 4.

${ }^{610} \mathrm{KOM}(2003) 356$ endgültig.

${ }^{611}$ Vgl. zur ursprünglichen Fassung von Art. 6 Abs. 2 lit. b) im Vorschlag der Europäischen Kommission für eine UGP-Richtlinie Fn. 605.

„- sofern es sich um eine eindeutige Verpflichtung handelt, deren Einhaltung nachprïfbar ist (...)“. 
jedoch nicht nur auf den Ausschluss dieser Art von Verhaltenskodizes. Vielmehr ist im Umkehrschluss jeder Verhaltenskodex, der keine Absichtserklärung darstellt, als solcher einzuordnen, der die Voraussetzungen von Art. 6 Abs. 2 lit. b) i) UGP-Richtlinie erfüllt. Denn das Tatbestandsmerkmal der eindeutigen Verpflichtung, deren Einhaltung nachprüfbar ist, ist nach dem Willen der Richtliniengeber nichts anderes als das Gegenstück zu einer Absichtserklärung. Insbesondere sollten sich aus dem Merkmal, dass die Einhaltung nachprüfbar zu sein hat, keinerlei weitergehende Anforderungen ergeben. Dies ergibt sich auch aus der Definition des Begriffs der festen Verpflicbtung aus dem Standpunkt des europäischen Parlaments, welche auf dieses überhaupt nicht eingeht. ${ }^{612}$

Im Rahmen der Feststellung, ob der Verstoß gegen eine Verpflichtung aus einem Verhaltenskodex eine Irreführung gemäß $₫ 5$ Abs. 1 Satz 2 Nr. 6 UWG darstellt, ist somit eine Analyse des jeweiligen Verhaltenskodexes dahingehend notwendig, ob er eine echte Verpflichtung auf bestimmte Verhaltensweisen enthält oder deren Einhaltung nur anstrebt. Dies ist im Hinblick auf das mit der UGPRichtlinie verfolgte Anliegen, den Verbraucherschutz zu stärken, insofern problematisch, als die Feststellung dieser Frage nur durch Prüfung des Verhaltenskodexes selbst möglich ist. Eine solche Prüfung nimmt ein durchschnittlicher Verbraucher jedoch erfahrungsgemäß nicht vor, da er sich dabei mit dem Verhaltenskodex in seiner Gesamtheit und dabei insbesondere mit genauen Formulierungen beschäftigen müsste. Dies ist — trotz der Möglichkeiten des mobilen Internets zumindest im Rahmen des alltäglichen Konsums nur schwer möglich. Aber auch sonst wird sich der durchschnittliche Verbraucher vielfach mit der Information des Unternehmers begnügen, er sei durch einen Verhaltenskodex verpflichtet. ${ }^{613}$ So beispielsweise bei Unternehmen, welche sich im Rahmen des UN Global Compact verpflichtet haben. Denn dieser verpflichtet die Unternehmen nicht zur Einhaltung der zehn Prinzipien, sondern lediglich zur Abgabe einer eindeutigen Unterstützungserklärung für den UN Global Compact. ${ }^{614}$ Somit stellt dieser für die beteiligten Unternehmen lediglich eine Absichtserklärung und damit keine verbindliche Verpflichtung i. S. d. $\int 5$ Abs. 1 Satz 2 Nr. 6 UWG dar, so dass ein Verstoß gegen seine Prinzipien keine irreführende geschäftliche Handlung sein kann. Im geschäftlichen Bereich dürfte eine solche Prüfung wohl zumindest dann üblich sein, wenn die Einhaltung bestimmter Verhaltensweisen für das prüfenden Unternehmen - möglicherweise aufgrund seines eigenen Verhaltenskodexes entscheidend für den Geschäftsabschluss sind. Nichtsdestotrotz lässt die eindeutige Formulierung der UGP-Richtlinie diesbezüglich keinen Spielraum, so dass nur

\footnotetext{
612 Vgl. Fn. 606.

613 Was aufgrund der Verpflichtung, sich zu bemühen, ja auch bei Absichtserklärungen der Wahrheit entspricht.

${ }^{614}$ Vgl. Kapitel 1C.III.2.
} 
bei Verhaltenskodizes mit entsprechend verbindlichen Verpflichtungen eine Irreführung über den Umstand aus $\int 5$ Abs. 1 Satz 2 Nr. 6 UWG in Betracht kommt.

\section{Hinweis auf Bindung an Verhaltenskodex}

Kumulativ zur verbindlichen Verpflichtung durch den Verhaltenskodex muss ein Hinweis des Unternehmers vorliegen, dass er an den Verhaltenskodex gebunden ist. Hierfür reicht jeder Hinweis auf die Abgabe des Verhaltenskodexes durch den Unternehmer aus, auch wenn der Wortlaut der Norm aufgrund der Bezugnahme auf ,diese Bindung“ so verstanden werden könnte, dass gerade auf die besondere Verbindlichkeit des Verhaltenskodexes ${ }^{615}$ hingewiesen werden müsste. Zwar würde der Wortlaut der Ursprungsnorm in der UGP-Richtlinie ${ }^{616}$ diese Auslegung ebenfalls zulassen, sie entspricht jedoch nicht dem Willen der Richtliniengeber. Denn Sinn und Zweck dieser Regelung ist die Sanktionierung von Verstößen gegen die Verpflichtungen aus Verhaltenskodizes in Fällen, in welchen zuvor durch einen Hinweis des Unternehmers ein besonderer Vertrauenstatbestand geschaffen wurde. ${ }^{617}$ Dies ergibt sich aus der Gesetzgebungshistorie von Art. 6 Abs. 2 lit. b) ii) UGP-Richtlinie, welcher seine letztlich gültige Fassung erst durch den gemeinsamen Standpunkt erhalten hat. ${ }^{618}$ Hintergrund war ebenfalls ein Änderungsantrag des Europäischen Parlaments ${ }^{619}$, die entsprechende Regelung im Entwurf der Kommission um die Voraussetzung zu erweitern, dass ,der Gewerbetreibende selbst mitteilt, dass er den Verbaltenskodex unterzeichnet hat. " ${ }^{20}$ Ein solcher Vertrauenstatbestand wird jedoch bei jedem Hinweis auf die Unterzeichnung eines Verhaltenskodexes geschaffen, da entsprechende Hinweise in aller Regel keinerlei Ausführungen über die inhaltliche Gestaltung des jeweiligen Verhaltenskodexes enthalten. ${ }^{621}$ Ausreichend ist somit beispielsweise der Hinweis eines Unternehmens in seinem Internetauftritt, durch einen Verhaltenskodex verpflichtet zu sein. ${ }^{622}$

${ }^{615}$ Vgl. Kapitel 5B.III.1.

616 Art. 6 Abs. 2 lit. ii) der UGP-Richtlinie lautet: „der Gewerbetreibende im Rabmen einer Geschäftspraxis darauf binweist, dass er durch den Kodex gebunden ist."

${ }^{617}$ So auch Dreyer, WRP 2007, 1294, 1300, die feststellt, dass dem Unternehmer, anders als bei $₫ 3$ Abs. 3 i. V. $\mathrm{m}$ Nr. 1 des Anhangs (zu $₫ 3$ Abs. 3 UWG), ,nicht der Hinweis auf die Kodexbindung als solches zum Vonvurf gemacht (wird, Ergänzung d. Verf.), sondern sein dazu im Widerspruch stehendes späteres Verhalten, durch das er das aus dem Hinweis resultierende Vertranen des Verkebrs enttäuscbt."

${ }^{618}$ Gemeinsamer Standpunkt (2005/C 38 E/01).

${ }^{619} \mathrm{KOM}$ (2004) 753 endgültig, S. 4.

${ }^{620}$ Standpunkt des Europäischen Parlaments zur UGP-Richtlinie, Art. 6 Abs. 2 lit. b) Spiegelstrich 3 (P5_TC1-COD(2003)0134). Zur Bedeutung des Begriffs der Unterzeichnung in diesem Zusammenhang vgl. Kapitel 5A.II.1.

${ }^{621}$ Vgl. hierzu Kapitel 5B.III.1. bzgl. der bereits mit dem Ausschluss von Absichtserklärungen einhergehenden Problematik im Bezug auf den Verbraucherschutz.

622 Vgl. hierzu Nr. 2 der Auflistung in Kapitel 4A. 
Aufgrund dieses Irreführungsvorwurfes kann es auch nicht von Bedeutung sein, ob der Verhaltenskodex aus irgendwelchen Gründen unwirksam ist und aus diesem Grund tatsächlich keine Bindung des Unternehmers an den Verhaltenskodex besteht. ${ }^{623}$ Denn trotz allem wurde durch den Hinweis beim Verkehr ein entsprechendes Vertrauen hervorgerufen. Zudem würde dies zu einer ungerechtfertigten Besserstellung eines Unternehmers, der eine Verpflichtung aus einem unwirksamen Verhaltenskodex nicht einhält, gegenüber einem Unternehmer führen, der eine Verpflichtung aus einem wirksamen Verhaltenskodex nicht einhält. Diese Besserstellung lässt sich insbesondere nicht mit dem Einwand rechtfertigen, dass ansonsten das Lauterkeitsrecht dem Unternehmer mit dem unwirksamen Verhaltenskodex die Erfüllung unwirksamer Verpflichtungen abverlangen würde. ${ }^{624}$

IV. Zusammenfassung

Mit $₫ 5$ Abs. 1 Satz 2 Nr. 6 UWG besteht seit Geltung des UWG 2008 erstmalig eine Regelung im UWG, die Verstöße gegen Verhaltenskodizes als irreführend einordnet. Liegt kein Verstoß gegen einen Verhaltenskodex vor, kommt eine Irreführung nach $\ 5$ Abs. 1 Satz 2 Nr. 6 UWG entgegen dessen Wortlaut und dem Willen des deutschen Gesetzgebers 625 nicht in Betracht. Dies gilt unabhängig davon, ob trotzdem eine Täuschungseignung gegeben ist. Denn die Norm ist entsprechend der Vorgaben der UGP-Richtlinie auszulegen, welche den Verstoß gegen einen Verhaltenskodex erfordert ${ }^{626}$, auf den sich der die geschäftliche Handlung tätigende Unternehmer zudem selbst verpflichtet haben muss. Eine Irreführung aufgrund eines Verstoßes gegen einen Verhaltenskodex nach $\ 5$ Abs. 1 Satz 2 Nr. 6 UWG scheidet somit ebenfalls aus, wenn der die geschäftliche Handlung tätigende Unternehmer nicht an dem Verhaltenskodex beteiligt ist. Zudem erfasst $₫ 5$ Abs. 1 Satz 2 Nr. 6 UWG nicht Verstöße gegen sämtliche Verhaltenskodizes i. S. d. $\$ 2$ Abs. 1 Nr. 5 UWG, sondern nur Verstöße gegen solche Verhaltenskodizes, die eine verbindliche Verpflichtung enthalten und nicht nur eine Absichtserklärung darstellen. Dieser Ausschluss von Absichtserklärungen steht jedoch in einem gewissen Widerspruch zum Zweck der UGP-Richtlinie, zu einem hohen Verbraucherschutzniveau beizutragen. ${ }^{627}$ Denn der durchschnittliche Verbraucher wird zumindest im Rahmen von Alltagsgeschäften nicht festzustellen können, ob ein Verhaltenskodex, auf welchen er von einem Unternehmer hingewiesen wird, eine verbindliche Verpflichtung i. S. d. $\ 5$ Abs. 1 Satz 2 Nr. 6 UWG

\footnotetext{
${ }^{623}$ A. A. Dreyer, WRP 2007, 1294, 1301.

${ }^{624} \mathrm{Vgl}$. Fn. 623.

${ }^{625}$ Begründung der Bundesregierung für den Gesetzentwurf zur Änderung des UWG vom 20.08.2008, BT.-Drs. 16/10145, S. 24.

626 Art. 6 Abs. 2 lit. b) UGP-Richtlinie erfasst ausdrücklich nur solche Geschäftspraktiken, die „die Nichteinhaltung von Verpflicbtungen" aus Verhaltenskodizes enthalten.

627 Art. 1 UGP-Richtlinie, vgl. Kapitel 2B.I.1.a.
} 
enthält oder ob es sich bei diesem lediglich um eine Absichtserklärung handelt. Des Weiteren setzt $\int 5$ Abs. 1 Satz 2 Nr. 6 UWG voraus, dass der Unternehmer darauf hingewiesen hat, dass er durch den Verhaltenskodex gebunden ist. Hierfür reicht jedoch jeder Hinweis des Unternehmers auf den Verhaltenskodex aus, so dass diese Voraussetzung in aller Regel vorliegen sollte. 



\section{Kapitel 6}

\section{Bewertung geschäftlicher Handlungen im Zusammenhang mit Verhaltenskodizes nach den sonstigen Regelungen des UWG zur Unlauterkeit}

Die Bewertung der Unlauterkeit geschäftlicher Handlungen im Zusammenhang mit Verhaltenskodizes ist nicht auf die vorstehend dargestellten Spezialregelungen ${ }^{628}$ beschränkt. Entsprechend sind sowohl die von den Spezialregelungen erfassten geschäftlichen Handlungen im Zusammenhang mit Verhaltenskodizes, als auch andere geschäftliche Handlungen im Zusammenhang mit Verhaltenskodizes an den sonstigen Regelungen des UWG zur Unlauterkeit zu messen.

$628 \mathrm{Vgl}$. Kapitel 5. 


\section{A. Unlauterkeit von Verhaltensweisen im Zusammenhang mit Verhaltenskodizes gemäß \$ 4 UWG}

$\int 4$ UWG enthält eine Auflistung von Beispielstatbeständen der Unlauterkeit. ${ }^{629}$ Er wurde im Rahmen der Neufassung des UWG im Jahr 2004 eingefügt, um die neu geschaffene Generalklausel zu präzisieren und dadurch eine größere Transparenz zu erreichen. ${ }^{630}$ Inhaltlich betreffen die aufgeführten Beispiele dabei im Wesentlichen die für die alte Generalklausel des UWG 1909 durch Richterrecht entwickelten Fallgruppen und kodifizieren diese. ${ }^{631}$ Systematisch ist $\int 4$ UWG im Unterschied zu der für $\int 5$ Abs. 1 UWG dargestellten Prüfungsreihenfolge ${ }^{632}$ kein der Irreführung entsprechendes subjektives Tatbestandsmerkmal zwischengeschaltet. Die Verwirklichung der in den Nr. 1 bis $11^{633}$ aufgeführten Tatbestände führt vielmehr unmittelbar zur Unlauterkeit einer geschäftlichen Handlung. Unzulässig sind die von $\int 4$ UWG umfassten geschäftlichen Handlungen jedoch ebenfalls nur bei kumulativem Vorliegen der Voraussetzungen des $\ 3$ Abs. 1 UWG. ${ }^{634}$

I. Hinweis auf Verhaltenskodex als Beeinträchtigung der

Entscheidungsfreiheit durch unangemessene, unsachliche Einflussnahme, $\int 4$ Nr. 13 . Alt. UWG

Der Beispieltatbestand Nr. $1{ }^{635}$ von \4 UWG erklärt geschäftliche Handlungen ${ }^{636}$ für unlauter, welche die Entscheidung von Verbrauchern und sonstigen Marktteilnehmern ${ }^{637}$ „durch Ausübung von Druck, in menschenverachtender Weise oder durch sonsti-

${ }^{629} \int 4$ UWG: „Unlauter handelt insbesondere, wer 1. \{... 11. \{... $\}^{“}$.

630 Regierungsentwurf und Begründung zum UWG vom 22.08.2004, BT.-Drs. 15/1487, S. 17.

631 Beater, Rn. 1057.

$632 \mathrm{Vgl}$. Kapitel 5B.I.

${ }^{633}$ Dabei regeln die Nr. 1 bis 6 Tatbestände, die sich ausdrücklich auf Verbraucher beziehen oder sich diesem Schutzzweck zuordnen lassen, während die Nummern 7 bis 10 Tatbestände im Verhältnis zwischen Unternehmern betreffen. Die abschließende Nr. 11 betrifft sodann allumfassend den sogenannten Rechtsbruch, vgl. Beater, Rn. 1057.

634 Vgl. hierzu Kapitel 5B.I.

635 Wortlaut der Norm: „Unlauter handelt insbesondere, wer

1. geschäftliche Handlungen vornimmt, die geeignet sind, die Entscheidung der Verbraucher oder sonstiger Marktteilnehmer durch Ausübung von Druck, in menschenverachtender Weise oder durch sonstigen unangemessenen unsachlichen Einfluss zu beeinträchtigen, "

636 Im Rahmen der Änderung des UWG zur Umsetzung der UGP-Richtlinie im Jahr 2008 wurde lediglich der Begriff der Wettbewerbshandlung durch den Begriff der geschäftlichen Handlung ersetzt (vgl. Regierungsentwurf und Begründung zum UWG vom 20.08.2008, BT.-Drs. 16/10145, S. 22) und damit der Anwendungsbereich entsprechend erweitert (vgl. Kapitel 4A.I).

637 Vom Wortlaut erfasst sind somit nur geschäftliche Handlungen gegenüber Verbrauchern und sonstigen Marktteilnehmern, jedoch ist $\int 4 \mathrm{Nr}$. 1 UWG darüber hinaus auch auf geschäftliche 
gen unangemessenen unsachlichen Einfluss $\{. .$.$\} beeinträchtigen" { }^{638}$ Dabei diente die dritte Alternative („Beeinträchtigung durch sonstige, unangemessene Einflussnahme ${ }^{6}$ ) insbesondere zur Kodifizierung der von der Rechtsprechung entwickelten Fallgruppe der gefühlsbetonten Werbung. ${ }^{639}$ Diese umfasst Werbung, welche ,edle Gefüble wie Mitleid, Hilfsbereitschaft und Mildtätigkeit des Umworbenen auszunutzen sucht $\{\ldots\}$ ".640, an die soziale Hilfsbereitschaft appelliert ${ }^{641}$ oder auf die besondere Art der Herstellung hinweist. ${ }^{642} \mathrm{Da}$ geschäftliche Handlungen im Zusammenhang mit Verhaltenskodizes $^{643}$ zumeist die soziale und ökologische Verantwortung der beteiligten Unternehmen hervorheben und entsprechend an die Verbraucher und sonstigen Marktteilnehmer appellieren, dies bei ihrer Entscheidung zu berücksichtigen, sind sie der Fallgruppe der gefühlsbetonten geschäftlichen Handlungen zuzuordnen.

\section{Rechtsprechung zur gefüblsbetonten Werbung}

Aufgrund der Tatsache, dass die Fallgruppe der gefühlsbetonten Werbung von der Rechtsprechung entwickelt wurde, sind deren Vorgaben für die Einordnung der geschäftlichen Handlungen als unzulässige, unsachliche Beeinträchtigungen der Entscheidungsfreiheit maßgeblich. Da die Rechtsprechung zu dieser Fallgruppe entscheidend geändert wurde, wird zum besseren Verständnis der Fallgruppe nachfolgend zunächst die frühere Rechtsprechung und im Anschluss daran die aktuelle Rechtsprechung dargestellt.

\section{a. Frühere Rechtsprechung zur gefühlsbetonten Werbung}

Nach der früheren Rechtsprechung bestand ein ,grundsätzliches Verbot der „gefühlsbetonten“ Werbung, soweit sie , $\{\ldots\}$, edle Gefühle wie Mitleid, Hilfsbereitschaft und Mildtätigkeit des Umworbenen auszunutzen sucht und damit in unsachlicher, wettbewerbswidriger Weise von den im Leistungswettbewerb für die Willensentschließung des Käufers wesentlichen Umständen ablenkt.“"644 Möglich

Handlungen zwischen Unternehmern auf verschiedenen Wirtschaftsstufen anwendbar, vgl. Regierungsentwurf und Begründung zum UWG vom 22.08.2004, BT.-Drs. 15/1487, S. 17.

638 Aus der Formulierung der Norm ergibt sich somit, dass die Beeinträchtigung der Entscheidung durch Ausübung von Druck sowie in menschenverachtender Weise Unterbegriffe des unangemessenen, unsachlichen Einflusses darstellen.

${ }^{639}$ Nach der neuen Terminologie des UWG: Fallgruppe der gefühlsbetonten geschäftlichen Handlungen.

${ }^{640}$ Urteil des BGH v. 14.11.1958 - I ZR 91/57 (OLG Celle) (Blindenseife) in GRUR 1959, 143, 144.

${ }^{641}$ Urteil des BGH v. 09.02.1995 - I ZR 44/93 (KG) (Arbeitsplätze bei uns) in GRUR 1995, 742, 743.

${ }^{642}$ Köhler in: Köhler/Bornkamm, UWG, \4 Rn. 1.232 ff., der dies als Werbung mit Appellen an die soziale Verantwortung bezeichnet, jedoch auf die Rechtsprechung zur gefühlsbetonten Werbung und auf die Zuordnung zu dieser Kategorie verweist.

${ }^{643} \mathrm{Vgl}$. Kapitel 4A.

${ }^{644}$ Urteil des BGH v. 14.11.1958 - I ZR 91/57 (OLG Celle) (Blindenseife) in GRUR 1959, 143, 144. 
war eine gefühlsbetonte Werbung nur dann, wenn ein sachlicher Zusammenhang zwischen dem für die Werbung genutzten gefühlsbetonten Element und der beworbenen Ware bestand.645 Einen solchen Sachzusammenhang erkannte die Rechtsprechung in einer der wenigen Ausnahmen ${ }^{646}$ für von körperbehinderten Künstlern geschaffene Postkarten an, da deren besondere Arbeitsweise ein besonderes Element der Leistung darstelle. ${ }^{647}$ Diese Rechtsprechung führte der BGH über Jahrzehnte fort und fasste auch Werbung mit Appellen an das soziale Verantwortungsgefühl beziehungsweise den Hinweis auf soziales Engagement des werbenden Unternehmens ${ }^{648}$ sowie dessen Forschungstätigkeit unter diese. ${ }^{649}$

b. Auslöser der Rechtsprechungsänderung zur gefühlsbetonten Werbung

Auslöser der Rechtssprechungsänderung des BGH war die BenettonEntscheidung des BVerfG, einer Grundsatzentscheidung des BVerfG zur Bedeutung des Grundrechts auf Meinungs- und Pressefreiheit im Wettbewerbsrecht. ${ }^{650}$ Dass sich das Urteil auch auf die Pressefreiheit im Wettbewerbsrecht bezieht ist dem Umstand geschuldet, dass entgegen dem sich aufgrund der Bezeichnung als Benetton-Entscheidung aufdrängenden ersten Eindruck nicht die Firma Benetton als Beschwerdeführer aufgetreten ist, sondern das Presseunternehmen Gruner \& Jahr als Herausgeber der Illustrierten „Stern“. Gegenstand der Verfassungsbeschwerden waren zwei Urteile des BGH, mit welchen Gruner \& Jahr die Veröffentlichung von drei Anzeigen der Firma Benetton untersagt wurde. Dabei betraf das erste Urteil651 Anzeigen, welche eine ölverschmierte Ente auf einem Ölteppich beziehungsweise arbeitende Kinder abbildeten und das zweite Urteil ${ }^{652}$ eine Anzeige mit einem unbekleideten menschlichen Gesäß, auf welchem sich ein Stempel mit dem Wortlaut „H.I.V. POSITIV“ befand. Alle Anzeigen enthielten darüber hinaus lediglich den Slogan der Firma Benetton „United Colors of Benetton“ in dem

\footnotetext{
${ }^{645}$ Scherer, GRUR 2008, 490, 491.

${ }^{646}$ Ebenfalls eine Ausnahme sollte gelten, wenn nicht der eigene Warenabsatz gefördert wurde, sondern quasi ein Hilfsgeschäft zur Finanzierung anerkannter karitativer Aufgaben vorlag, vgl. Urteil des BGH v. 16.01.1976 - I ZR 32/75 (OLG Hamburg) (UNICEF-Postkarte) in GRUR 1976, 308, 309.

${ }^{647}$ Urteil des BGH v. 11.11.1958 - I ZR 179/57 (OLG München) (Künstlerpostkarten) in GRUR 1959, 277, $279 \mathrm{f}$.

${ }^{648}$ Urteil des BGH v. 09.02.1995 - I ZR 44/93 (KG) (Arbeitsplätze bei uns) in GRUR 1995, 742, 743.

${ }^{649}$ Urteil des BGH v. 25.03.1999 - I ZR 88/97 (Stuttgart) (Generika-Werbung) in GRUR 1999, $1100 \mathrm{ff}$.

${ }^{650}$ Urteil des BVerfG vom 12.12.2000 - 1 BvR 1762/95; 1 BvR 1787/95 (Benetton) in GRUR 2001, $170 \mathrm{ff}$.

${ }^{651}$ Urteil des BGH v. 06.07.1995 - I ZR 110/93 (LG Frankfurt a. Main) (Kinderarbeit) = NJW 1995, $2490 \mathrm{ff}$.

${ }^{652}$ Urteil des BGH v. 06.07.1995 - I ZR 180/94 (Frankfurt a. Main) (H.I.V.-Positiv) = NJW 1995, $2492 \mathrm{ff}$.
} 
üblichen Schriftzug. Der BGH begründete die Unzulässigkeit der Werbemaßnahmen jeweils damit, dass die Darstellung des Elends der Welt sittenwidrig sei, wenn mit dem Mitleid und Schrecken der Betrachter der Name und das Kennzeichen eines Unternehmens bekanntgemacht werden soll. ${ }^{653}$

Das BVerfG erkannte jedoch in beiden Urteilen des BGH einen Verstoß gegen Art. 5 Abs. 1 Satz 2 1. Alt. GG und hob diese auf. Zur Begründung führte das BVerfG an, dass einem Presseorgan die Veröffentlichung einer fremden Meinungsäußerung nicht verboten werden könne, wenn dem Meinungsträger selbst die Äußerung und Verbreitung zu gestattet sei. ${ }^{654}$ Im Anschluss daran führt das BVerfG aus, dass die Anzeigen der Firma Benetton grundsätzlich eine Meinungsäußerung im Schutzbereich des Art. 5 Abs. 1 Satz 1 GG darstellen ${ }^{65}$ und der BGH die Bedeutung und Tragweite der Meinungsfreiheit verkannt habe. ${ }^{656}$ Denn entgegen der Ansicht des $\mathrm{BGH}^{657}$ erkannte das BVerfG keine hinreichend gewichtigen Gemeinwohlbelange oder schutzwürdige Rechte oder Interessen Dritter, welche für Einschränkungen des nach ständiger Rechtsprechung für eine freiheitlich demokratische Staatsordnung schlechthin konstituierenden Rechts auf freie Meinungsäußerung ${ }^{658}$ notwendig sind. ${ }^{659}$ Insbesondere erkannte das BVerfG solche nicht in der vom BGH angeführten Begründung, dass eine Werbung, welche die Darstellung schweren Leids von Menschen und Tieren zur Erregung von Mitleid und zur Solidarisierung der Verbraucher mit dem Unternehmen bezüglich der Ablehnung solchen Leids ausnutze, sittenwidrig sei. ${ }^{660}$ Zwar bezeichnete das BVerfG dieses Sittenwidrigkeitsurteil als „durchaus billigenswert“, erachtete es jedoch als nicht ohne weiteres erkennbar, dass mit diesem auch hinreichend gewichtige öffentliche und private Belange geschützt würden. ${ }^{661}$

Unter Bezugnahme auf die Benetton-Entscheidung ${ }^{662}$ stellte das BVerfG kurze Zeit später im Rahmen einer weiterer Verfassungsbeschwerde erneut einen Verstoß gegen das Grundrecht der Meinungsfreiheit durch ein sich auf das Verbot der gefühlsbetonten Werbung stützendes Urteil des BGH fest. ${ }^{663}$ Gegenstand des Ursprungsverfahrens war in diesem Fall eine Werbung in Printmedien, in welcher der Werbende und Beklagte des Verfahrens darauf hinwies, dass er die Aktions-

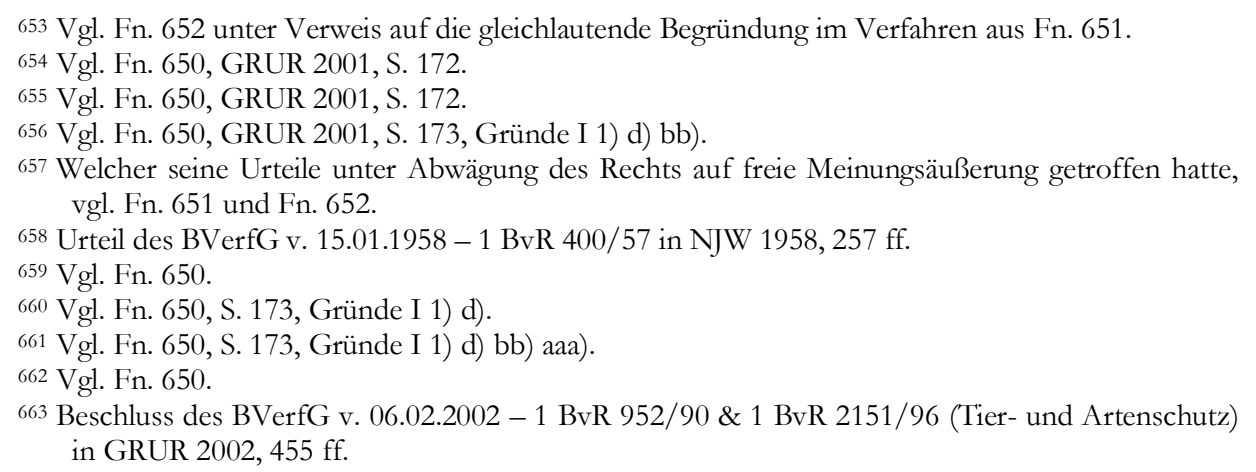


gemeinschaft Artenschutz e. V. unterstütze. ${ }^{664}$ Dabei stellte das BVerfG klar, dass es dem Kaufinteressenten frei stehe, auf Grund welcher Motive er sich zum Kauf anregen lassen wolle, soweit keine Irreführung vorliege. 665

\section{c. Aktuelle Rechtsprechung zur gefühlsbetonten Werbung}

Nachdem der BGH in Sachen Benetton auch nach der Aufhebung seiner Urteile und der Zurückverweisung durch das BVerfG zunächst weiter auf der Unzulässigkeit nach dem UWG und den damit bestehenden Unterlassungsansprüchen beharrte, gab er mit der Entscheidungstroika Artenschutz ${ }^{666}$, Regenwaldprojekt I ${ }^{667}$ und Regenwaldprojekt II ${ }^{668}$ seine bisherige Rechtsprechung zur gefühlsbetonten Werbung auf. Zur Begründung berief sich der $\mathrm{BGH}^{669}$ auf den damals neugefassten $\int 4$ Nr. 1 UWG ${ }^{670}$, welcher die Fallgruppe der gefühlsbetonten Werbung im neuen UWG regele. ${ }^{671}$ Aus diesem ergebe sich, dass es wettbewerbsrechtlich grundsätzlich unbedenklich sei, „wenn sich die Werbung nicht auf Sachangaben, insbesondere über die Eigenschaften und den Preis der Erz̨eugnisse, beschränkt, sondern Gefüble anspricht." 672 Entsprechend könne ,eine Werbemaßnabme nicht schon als unlauter angeseben werden, wenn das Kaufinteresse durch Ansprechen des sozialen Verantwortungsgefühls, der Hilfsbereitschaft, des Mitleids oder des Umweltbewusstseins geweckt werden soll, obne das ein sachlicher Zusammenhang zwischen dem in der Werbung angesprochenen Engagement und der beworbenen Ware besteht, und nur zielbewusst und planmäßig an die Gefühle appelliert wird, um diese im eigenen wirtschaftlichen Interesse als entscheidende Kaufmotivation auszunutzen. "“73 Im Hinblick auf die Bedeutung dieser Feststellungen für die Fallgruppe der gefühlsbetonten Werbung stellte der BGH zudem ausdrücklich fest: „An der gegentei-

\footnotetext{
${ }^{664}$ Zuvor war der Beklagte sowohl vor dem LG Stuttgart als auch in der Berufung vor dem OLG Stuttgart (Urteil des OLG Stuttgart v. 01.12.1995 - 2 U 72/95 in NJWE-WettbR 1996, 144 ff.) mit dem Verweis auf die Unzulässigkeit der gefühlsbetonten Werbung unterlegen. Die Revision gegen das Urteil des OLG Stuttgart hatte der BGH nicht angenommen, da er keine grundsätzliche Bedeutung sah und die Revision keine Aussicht auf Erfolg habe.

665 Vgl. Fn. 663, GRUR 2002, 455, 457.

666 Urteil des BGH v. 22.09.2005 - I ZR 55/02 (OLG Stuttgart) (Artenschutz) in GRUR 2006, S. $75 \mathrm{ff}$.

667 Urteil des BGH v. 26.10.2006 - I ZR 33/04 (OLG Hamm) (Regenwaldprojekt I) in GRUR 2007, S. $247 \mathrm{ff}$.

${ }^{668}$ Urteil des BGH v. 26.10.2006 - I ZR 97/04 (LG Gießen) (Regenwaldprojekt II) in GRUR 2007, S. $251 \mathrm{ff}$.

${ }^{669}$ Vgl. Fn. 666, GRUR 2006, S. 75, 76, Tz. 18.

${ }^{670}$ Zum Wortlaut vgl. Fn. 635, wobei zum Entscheidungszeitraum statt auf geschäftliche Handlungen noch auf Wettbewerbshandlungen abgestellt wurde.

${ }^{671}$ Vgl. Fn. 666, Tz. 15 ff.

672 Vgl. Fn. 666, GRUR 2006, S. 75, 76, Tz. 17.

673 Vgl. Fn. 666, GRUR 2006, S. 75, 76, Tz. 18.
} 
ligen Beurteilung in früheren Senatsentscheidungen (unter dem Gesichtspunkt der sogenannten gefüblsbetonten Werbung) ist nicht mehr festzubalten. "674

\section{Verbliebener Anwendungsbereich des $\int 4$ Nr. 13 . Alt. UWG}

Mit der Aufgabe der Rechtsprechung zur gefühlsbetonten Werbung hat die Regelung des $\int 4$ Nr. 13 . Alt. UWG bereits unter Geltung des UWG 2004 einem Großteil ihres Anwendungsbereiches verloren. Denn eine unangemessene unsachliche Einflussnahme i. S. d. Norm wollte der BGH nur noch dann anerkennen, „wenn der Einfluss ein solches Ausmaß erreicht, dass er die freie Entscheidung des Verbrauchers zu beeinträchtigen vermag. " 775 Unabhängig von dieser Auslegungsmaßgabe des BGH hat die Auslegung der Norm seit In-Kraft-Treten der UGP-Richtlinie und der damit einhergehenden Vollharmonisierung des europäischen Lauterkeitsrechts im Hinblick in jedem Fall richtlinienkonform zu erfolgen. Dies gilt aufgrund eines fehlenden abweichenden Willens des deutschen Gesetzgebers nicht nur für geschäftliche Handlungen gegenüber Verbrauchern, sondern auch für solche gegenüber sonstigen Marktteilnehmern sowie zwischen Unternehmern.

Die UGP-Richtlinie kennt jedoch weder die Begrifflichkeiten ,sachlich“ beziehungsweise „unsachlich“ als Merkmal der Einordnung geschäftlicher Praktiken als unlauter, sondern lediglich im Zusammenhang mit sachlichen richtigen beziehungsweise sachlich falschen Angaben ${ }^{676}$, Behauptungen ${ }^{677}$ oder Informationen $^{678}$, noch enthält sie eine dem $\int 4$ Nr. 1 UWG entsprechende Regelung. ${ }^{679}$ Inhaltlich stehen dieser jedoch die Regelungen zur Beeinflussung von Verbrauchern durch Belästigung, Nötigung, einschließlich der Anwendung körperlicher Gewalt und durch unzulässige Beeinflussung aus den Artikeln 8 und 9 UGPRichtlinie nahe. ${ }^{60}$ Denn sowohl $₫ 4$ Nr. 1 UWG als auch Artikel 8 UGPRichtlinie betreffen die Beeinträchtigung der Entscheidungsfreiheit 681 der Verbraucher ${ }^{682}$ in besonders schwerwiegender, eventuell sogar strafrechtlich relevanter Art und Weise und erklären entsprechende Geschäftspraktiken beziehungswei-

\footnotetext{
${ }^{674}$ Vgl. Fn. 666, GRUR 2006, S. 75, 76, Tz. 18.

675 Vgl. Fn. 667, GRUR 2007, 247, 249, Tz. 21.

676 Art. 6 Abs. 1 UGP-Richtlinie.

677 Anlage I Nr. 12 UGP-Richtlinie.

678 Anlage I Nr. 18 UGP-Richtlinie.

${ }^{679}$ Die UGP-Richtlinie enthält eine Auflistung von Beispielen für unlautere Handlungen nur im Rahmen ihrer Anlage I, wobei diese im Regelfall deutlich konkreter formuliert sind.

${ }^{680}$ Stuckel in: Harte-Bavendamm/Henning-Bodewig, UWG, $\ 4$ A, Rn. 2 ff.

${ }^{681}$ Bzw. der Verhaltensfreiheit, Art. 8 UGP-Richtlinie.

${ }^{682}$ So betrifft $\int 4$ Nr. 1 UWG geschäftliche Handlungen, welche „die Entscheidungsfreibeit der Verbraucher $\{\ldots\}$ beeinträ̈btigen" und Art. 8 UGP-Richtlinie eine Geschäftspraktik, welche „die Entscheidungs- oder Verbaltensfreibeit des Durchschnittsverbrauchers $\{\ldots\}$ beeinträchtigt".
} 
se geschäftliche Handlungen als unlauter. ${ }^{683}$ Darüber hinaus sind auch die für eine solche Beeinträchtigung genannten konkret angeführten Mittel ${ }^{684}$ zwar nicht deckungsgleich, jedoch hinsichtlich ihres Unrechtsgehaltes vergleichbar. Zudem verwenden beide Normen neben den konkreten Rechtsbegriffen als Auffanglösung jeweils einen unbestimmten Rechtsbegriff. Auch wenn sich aus der Formulierung der UGP-Richtlinie, anders als im UWG für den unsachlichen, unangemessenen Einfluss, nicht ergibt, dass der unbestimmte Rechtsbegriff der unzulässigen Beeinflussung als Oberbegriff der konkret genannten Verhaltensweisen fungiert, legt dessen Legaldefinition als „Ausnutzung einer Machtposition gegenüber dem Verbraucher zur Ausübung von Druck, auch obne die Anwendung oder Androbung von körperlicher Gewalt, in einer Weise, die die Fähigkeit des Verbrauchers zu einer informierten Entscheidung wesentlich einschränkt"685 dies nahe. Denn hierunter kann sowohl die Belästigung als auch die Nötigung gefasst werden. Aus diesem Grund ist \4 Nr. 1 3. Alt. UWG insoweit richtlinienkonform i. S. d. Art. 8 UGP-Richtlinie auszulegen, als eine unangemessene unsachliche Einflussnahme dem Unrechtsgehalt der in diesem genannten Verhaltensweisen beziehungsweise der Definition der unzulässigen Beeinflussung in Art. 2 lit. j) UGP-Richtlinie entsprechen muss. Eine solche Art der Beeinflussung kann jedoch bei der Fallgruppe der gefühlsbetonten geschäftlichen Handlungen nicht erkannt werden, so dass diese gänzlich aufzugeben ist.

\section{Zusammenfassung}

Geschäftliche Handlungen im Zusammenhang mit Verhaltenskodizes sowie speziell die Werbung mit Verhaltenskodizes und damit mit dem besonderen Engagement eines Unternehmens fallen unter die von der Rechtsprechung entwickelte Fallgruppe der gefühlsbetonten Werbung und sind damit grundsätzlich zulässig. Dies gilt unabhängig davon, ob im Rahmen einer Produktwerbung auf die Verhaltenskodex hingewiesen wird oder ob die geschäftliche Handlung nur aus dem Hinweis auf den Verhaltenskodex besteht. Zwar beeinflussen entsprechende geschäftliche Handlungen die Entscheidungsfreiheit der Verbraucher, jedoch nicht in unangemessener Weise. Sie sind somit nicht unlauter i.S. d. \4 Nr. 1 3. Alt. UWG. Bei der heutigen Schwemme an Hinweisen von Unternehmen auf ihr gutes Tun und Handeln sowie die nachhaltige Herstellung ihrer Produkte wird sich diese Frage für viele unbeteiligte Beobachter der Marktgeschehens daher gar nicht mehr stellen. Entsprechend kann leicht in Vergessenheit geraten, dass sich

\footnotetext{
$683 \int 4$ Nr. 1 UWG direkt. Art. 8 UGP-Richtlinie bestimmt die betroffenen geschäftlichen Praktiken selbst nur als aggressiv, jedoch sind aggressive Geschäftspraktiken nach Art. 8 UGP-Richtlinie aufgrund von Art. 5 Abs. 4 lit. b) UGP-Richtlinie unlauter.

${ }^{684}$ In $\int 4$ Nr. 1 UWG ,durch Ausübung von Druck“" und ,in menschenveracbtender Weise“, in Art. 8 UGPRichtlinie „Belästigung" und „Nötigung, einschließlich der Anwendung körperlicher Gewalt".

685 Art. 3 lit. j) UGP-Richtlinie.
} 
die Rechtsprechung lange Zeit sehr schwer getan hat, entsprechende Verhaltensweisen zu tolerieren.

II. Gezielte Behinderung von Mitbewerbern durch Verhaltenskodizes, $\int 4$ Nr. 10 UWG

Der Beispieltatbestand aus $\int 4$ Nr. $10 \mathrm{UWG}^{686}$ betrifft geschäftliche Handlungen, die darauf gerichtet sind, einen Mitbewerber gezielt zu behindern. Dabei ist unter einer Behinderung eines Mitbewerbers die Beeinträchtigung seiner wettbewerblichen Entfaltungsmöglichkeiten zu verstehen ${ }^{687}$, wobei der Begriff der wettbewerblichen Entfaltungsmöglichkeiten weit zu fassen ist. ${ }^{688}$ Dabei ist eine Behinderung dann als gezielt anzusehen, wenn die Maßnahme bei objektiver Würdigung aller Umstände in erster Linie nicht auf die Förderung der eigenen wettbewerblichen Entfaltung, sondern auf die Beeinträchtigung der wettbewerblichen Entfaltung eines Mitbewerbers gerichtet ist. ${ }^{689}$

Eine solche gezielte Behinderung kommt im Zusammenhang mit Verhaltenskodizes in Betracht, wenn eine Vielzahl von Unternehmen einer Branche oder zumindest die größten Unternehmen einer Branche einen Verhaltenskodex aufstellen, der Anforderungen an die fachliche Sorgfalt in dieser Branche definiert, mit denen beispielsweise bestimmte Werbemaßnahmen eines anderen Branchenunternehmens nicht vereinbar sind. ${ }^{690}$

Fraglich ist jedoch, ob in diesen Fällen eine Behinderung vorliegt. Dies wäre abzulehnen, wenn eine Behinderung nur bei der realistischen Möglichkeit einer lauterkeitsrechtlichen Sanktionierung beispielsweise in Form einer Unterlassungsverpflichtung anzunehmen wäre. Denn lauterkeitsrechtlich hat ein Verstoß gegen einen Verhaltenskodex nur für denjenigen unmittelbare Konsequenzen, der sich selbst auf diesen verpflichtet hat ${ }^{691}$, da aufgrund der aktuellen Rechtsprechung eine lauterkeitsrechtliche Sanktionierung eines Verstoßes gegen einen von einem Unternehmen selbst nicht unterzeichneten Verhaltenskodexes aufgrund der Generalklauseln des $\int 3$ UWG faktisch nicht mehr in Betracht kommt. Grund hierfür

686 Wortlaut der Norm:

"Unlauter handelt insbesondere, wer

10. Mitbewerber gezielt behindert;"

${ }^{687}$ Urteil des BGH v. 17.05.2001 - I ZR 216/99 (Hamburg) in GRUR 2001, 1061, 1062.

688 Ausweislich des Urteils des BGH v. 24.06.2004 - I ZR 26/02 (KG) in GRUR 2004., 877, 879 umfasst er "alle Wettbewerbsparameter [...] wie Absatz, Bezug, Werbung, Produktion, Finanzierung und Personal."

${ }^{689}$ BGH, Urteil v. 20.01.2005 - I ZR 29/02 (Düsseldorf) (The Colour of Elegance) in WRP 2005, 881, 884, Köhler in: Nornkamm/Köhler, UWG, \ 4, Rn. 10.7.

${ }^{690}$ Nach Ansicht von Sosnitza in: FS für Griss, S. 515 f., werden Verhaltenskodizes "gan₹ offensicbtlich auch immer wieder dazu eingesetət, um besonders wettbewerbsaktive Konkurrenten zu zügeln [...]."

${ }^{691}$ Denn $\int 5$ Abs. 1 Nr. 6 UWG betrifft nur die Nichteinhaltung eigener Verhaltenskodizes, vgl. Kapitel 5B.III. 
ist, dass Verhaltenskodizes auf die dort wesentliche Einordnung einer geschäftlichen Handlung als unlauter beziehungsweise als Verstoß gegen die fachliche Sorgfalt keinen direkten Einfluss, sondern können lediglich als Auslegungshilfen herangezogen werden können. ${ }^{692}$

Nach Ansicht des Verfassers führt dies jedoch nicht zur Ablehnung einer Behinderung i. S. d. \4 Nr.10 UWG. Schon die indirekte Wirkung eines solchen Verhaltenskodexes hindert die Unternehmen daran, sich im Rahmen der rechtlich zulässigen Möglichkeiten wettbewerblich zu entfalten. So beispielsweise aufgrund der unabhängig davon bestehenden Möglichkeit einer rechtlichen Auseinandersetzung oder einer aufgrund der Nichteinhaltung der "Branchenstandards" negativen Berichterstattung in der Presse.

Inwieweit eine durch einen solchen Verhaltenskodex eingetretene Behinderung gezielt ist, muss dabei in jedem Einzelfall entschieden werden. Ein eindeutiges Indiz für eine solche Zielgerichtetheit wäre dabei in jedem Fall, wenn der jeweilige Verhaltenskodex offensichtlich und nachträglich auf ein bestimmtes Verhalten eines Konkurrenzunternehmens ausgerichtet wäre.

III. Verstoß gegen eine gesetzliche Vorschrift, \4 Nr. 11 UWG

Der Beispieltatbestand aus $\int 4$ Nr. 11 UWG $^{693}$ betrifft den Verstoß gegen eine gesetzliche Vorschrift, die zumindest auch das Marktverhalten regelt. Obwohl nicht ausdrücklich erwähnt, werden selbstverständlich nur solche Verstöße erfasst, die im Rahmen einer geschäftlichen Handlung erfolgen. ${ }^{694}$

\section{Richtlinienkonforme Auslegung}

Wie das gesamte UWG ist auch $\int 4$ Nr. 11 UWG richtlinienkonform auszulegen. Die UGP-Richtlinie enthält jedoch keine entsprechende Regelung über die Unlauterkeit von Geschäftspraktiken, die gegen gesetzliche Vorschriften verstoßen. Unabhängig davon ist \ 4 Nr. 11 UWG aber insoweit anwendbar, als die UGPRichtlinie aufgrund der Öffnungsklauseln in ihrem Art. 3 zurücksteht. ${ }^{695}$ So lässt die UGP-Richtlinie nach ihrem Art. 3 Abs. 3 Rechtsvorschriften der Gemeinschaft oder der Mitgliedstaaten in Bezug auf die Gesundheits- und Sicherheitsaspekte von Produkten ausdrücklich unberührt. Entsprechend bleibt \4 Nr. 11 UWG für diese Bereiche betreffende Marktverhaltensregelungen anwend-

\footnotetext{
692 Vgl. Kapitel 6D.II.

693 Wortlaut der Norm:

„Unlauter handelt insbesondere, wer

11. einer gesetzlichen Vorschrift zuwiderhandelt, die auch dazu bestimmt ist, im Interesse der Marktteilnehmer das Mark.tverbalten zu regeln."

694 Vgl. Kapitel 4A.

695 Vgl. Kapitel 2B.I.1.a.
} 
bar. ${ }^{696}$ Gleiches gilt aufgrund von Art. 3 Abs. 8 UGP Richtlinie für Standesregelungen. ${ }^{697}$ Zudem bestimmt die UGP-Richtlinie in Art. 3 Abs. 4, dass im Falle der Kollision ihrer Rechtsvorschriften mit anderen Rechtsvorschriften der Gemeinschaft, die besondere Aspekte unlauterer Geschäftspraktiken regeln, die anderen Rechtsvorschriften denen der UGP-Richtlinie vorgehen. Unter Verweis auf diesen Vorrang hat der BGH zu Recht erkannt, dass deutsche Regelungen, die europäische Richtlinien umsetzen, weiterhin wettbewerbsrechtlich unter Berufung auf $\int 4$ Nr. 11 UWG durchgesetzt werden könnten. ${ }^{698}$

Aber auch innerhalb des Anwendungsbereiches der UGP-Richtlinie ist $\int 4$ Nr. 11 UWG anwendbar. So wenn durch die gesetzliche Vorschrift Informationspflichten durchgesetzt werden, die auf dem Gemeinschaftsrecht beruhen. Denn deren Durchsetzung erlaubt die UGP-Richtlinie ausdrücklich. ${ }^{699}$ Dass eine Sanktionierung nach dem System der UGP-Richtlinie im Wege einer Irreführung durch Unterlassen erfolgt, steht der grundsätzlichen Anwendbarkeit des $\ 4$ Nr. 11 UWG nicht entgegen. Denn die Art und Weise der Umsetzung einer Richtlinie obliegt allein den Mitgliedstaaten. Jedoch ist die Anwendung des $\int 4$ Nr. 11 UWG bezüglich geschäftlicher Handlungen gegenüber Verbrauchern auf den Regelungsumfang der UGP-Richtlinie beschränkt.

\section{Begriff der gesetzlichen Vorschrift}

Unabhängig von einer Einschränkung des Anwendungsbereiches des $\int 4$ Nr. 11 UWG durch eine richtlinienkonforme Auslegung ist für die Anwendung der Norm auf geschäftliche Handlungen im Zusammenhang mit Verhaltenskodizes und insbesondere solchen, die einen Verstoß gegen einen Verhaltenskodex beinhalten, der Begriff der gesetzlichen Vorschrift entscheidend. Denn nur wenn Verhaltenskodizes eine solche gesetzliche Vorschrift i. S.d. \4 Nr. 11 UWG darstellen, wäre ein Verstoß gegen sie als unlauter einzuordnen.

Der Begriff der gesetzlichen Vorschrift ist weder durch das UWG definiert, noch enthält die Gesetzesbegründung zum UWG 2004, mit welchem die Norm eingeführt wurde, Hinweise zur Bestimmung des Begriffs. ${ }^{700}$ Der BGH hat den Begriff ohne besondere Begründung unter Ableitung aus Art. 2 EGBGB als jede Rechts-

\footnotetext{
${ }^{696}$ Urteil des BGH v. 15.01.2009 - I ZR 141/06 (OLG Köln) (Überregionaler Krankentransport) in GRUR 2009, 881, 883, Tz. 16.

${ }^{697}$ Urteil des BGH v. 09.07.2009 - I ZR 13/07 (OLG Celle) (Brillenversorgung) in GRUR 2009, 977, 978, Tz, 12.

${ }^{698}$ Urteil des BGH v. 22.04.2009 - I ZR 216/06 (OLG Dresden) (Internet-Videorekorder) in GRUR 2009, 845, 848 f., Tz. 38.

${ }^{699}$ Ohly in: Piper/Ohly/Sosnitza, UWG, \ 11, Rn. 11.7.

700 Begründung der Bundesregierung für den Gesetzentwurf des UWG vom 22.08.2003, BT.Drs. 15/1487.
} 
norm bestimmt. ${ }^{701}$ Dieser Begriffsbestimmung hat sich die Literatur trotz der fehlenden Begründung angeschlossen. ${ }^{702}$ Voraussetzung einer Rechtsnorm nach Art. 2 EGBGB ist, dass die Regelung normative und tatsächliche Geltung besitzt. ${ }^{703}$ Dies bedeutet, dass ihre Verbindlichkeit von staatlicher Autorität gewollt und ihre Durchsetzung von dieser zumindest in der Mehrzahl der Fälle gewährleistet wird. ${ }^{704}$ Indiz für die normative Geltung ist dabei die staatliche Durchsetzung oder Sanktionierung von Verstößen. ${ }^{705}$ Nicht ausreichend ist dahingegen, wenn die Regelung nur aufgrund von ethischen oder psychischen Gründen befolgt wird.706 Entsprechend können Verhaltenskodizes i. S. v. \ 2 Abs. 1 Nr. 5 UWG nicht als gesetzliche Vorschriften i. S. d. \ 4 Nr. 11 UWG anerkannt werden. ${ }^{707}$

Dahingegen handelt es sich bei $\int 312 \mathrm{a}$ Abs. 2 BGB i. V. m. Art. 246a \ 1 Nr. 10 EGBGB ${ }^{708}$ zweifellos um eine solche gesetzliche Vorschrift. Da diese Unternehmen gegenüber Verbrauchern bei außerhalb von Geschäftsräumen geschlossenen Verträgen und Fernabsatzverträgen zur Information über gegebenenfalls bestehende einschlägige Verhaltenskodizes gemäß Art. 2 lit. f. UGPRichtlinie und der Möglichkeit des Bezugs von Exemplaren dieser Verhaltenskodizes einen Verstoß gegen eine gesetzliche Vorschrift verpflichtet, ist das Unterlassen $^{709}$ dieser Information gemäß $\int 4$ Nr. 11 UWG unlauter. ${ }^{710}$

\section{Zusammenfassung}

Verstöße gegen Verhaltenskodizes sind nicht nach \ 4 Nr. 11 UWG unlauter, da Verhaltenskodizes keine rechtlichen Vorschriften i. S. d. Norm darstellen. Allerdings stellt das Unterlassen der Informationen, dass ein Verhaltenskodex besteht und wie ein Exemplar von diesem erlangt werden kann, einen nach $\int 4$

${ }^{701}$ Urteil des BGH v. 21.07.2005 - I ZR 170/02 (OLG München) (Friedhofsruhe) in GRUR 2005, 960, 961.

702 Vgl. hierzu die Einschätzung der gängigen Kommentarliteratur, u.a. Ohly in: Piper/Ohly/Sosnitza, UWG, \11, Rn. 11,12, Köhler in: Köhler/Bornkamm, UWG, \4, Rn. 11.24 sowie eingehend von Walter, S. 53 ff.

${ }^{703}$ Merten in: Staudinger, BGB und EGBGB, Art. 2, Rn. 7.

704 Vgl. Fn. 703, Rn. 7.

705 Vgl. Fn. 703, Rn. 8.

706 Vgl. Fn. 703, Rn. 7.

${ }^{707}$ Urteil des BGH v. 09.09.2010 - I ZR 157/08 (FSA-Kodex) (OLG München) in GRUR 2011, 431, 432, Ohly in: Piper/Ohly/Sosnitza, UWG, \11, Rn. 11.13, Köhler in: Köhler/Bornkamm, UWG, \ 4, Rn. 11.30, Schmidhuber, WRP 2010, 593, 595, Beater, Rn. 2496.

${ }^{708}$ In der ab dem 13.06.2014 geltenden Fassung. Gesetz zur Umsetzung der Verbraucherrechterichtlinie und zur Änderung des Gesetzes zur Regelung der Wohnungsvermittlung, BGBl. 2013, Teil I, Nr. 58, S. 3644 (n. F. \312a BGB) bzw. S. 3655 (n. F.Art. 246a EGBGB).

709 Vgl. zur Irreführung durch Unterlassung Kapitel 6C.

710 Alexander, WRP 2014, 501, 507. 
Nr.11 UWG unlauteren Verstoß gegen die gesetzliche Vorschrift \312a Abs. 2 BGB i. V. m. Art. 246a \ 1 Nr. 10 EGBGB 711 dar.

\section{B. Irreführung durch aktives Handeln, $\$ 5$ UWG}

Für die Einordnung geschäftlicher Handlungen als irreführend aufgrund eines aktiven Handelns stellt $\int 5$ Abs. 1 Satz 2 UWG die zentrale Regelung dar. Er bestimmt geschäftliche Handlungen als irreführend, wenn sie unwahre Angaben oder sonstige zur Täuschung geeignete Angaben ${ }^{712}$ über die in seinen Nummern 1 bis 7 genannten Umstände ${ }^{713}$ enthalten. ${ }^{714}$ Entsprechend unterliegen auch sämtliche Angaben im Zusammenhang mit Verhaltenskodizes in geschäftlichen Handlungen den Maßgaben des $\int 5$ Abs. 1 Satz 2 UWG, soweit sie nicht den Verstoß gegen einen Verhaltenskodex betreffen. ${ }^{715}$ Wann aufgrund eines Verstoßes gegen einen Verhaltenskodex eine Irreführung vorliegt, bestimmt sich allein durch $\int 5$ Abs. 1 Satz 2 Nr. 6 UWG. ${ }^{716}$ Denn andernfalls könnte beispielsweise der Verstoß gegen einen als Absichtserklärung einzuordnenden Verhaltenskodex irreführend sein, obwohl dies eindeutig dem Willen der Richtliniengeber und des Gesetzgebers widersprechen würde. ${ }^{717}$ Gleichfalls wäre eine darüber hinausgehende Sperrwirkung des $\int 5$ Abs. 1 Satz 2 Nr. 6 UWG nicht mit dem Willen der Richtliniengeber zu vereinbaren, da mit dieser Regelung nur der Grundstein für eine Stärkung des Vertrauens der Verbraucher in Verhaltenskodizes gelegt werden

${ }^{711}$ In der ab dem 13.06.2014 geltenden Fassung. Gesetz zur Umsetzung der Verbraucherrechterichtlinie und zur Änderung des Gesetzes zur Regelung der Wohnungsvermittlung, BGBl. 2013, Teil I, Nr. 58, S. 3644 (n. F. $\int 312$ a BGB) bzw. S. 3655 (n. F.Art. 246a EGBGB).

712 Zum Begriff der Angabe vgl. Kapitel 5A.II.1.

713 Die Frage, ob die aktive Irreführung auf die in $\ 5$ Abs. 1 Satz 2 Nr. 1 bis 7 UWG genannten Umstände beschränkt ist, ist im Rahmen dieser Arbeit nicht von Belang, da sämtliche Angaben im Zusammenhang mit Verhaltenskodizes unter diese gefasst werden können. Für eine abschließende Regelung durch $\int 5$ Abs. 1 Satz 2 UWG: Nordemann, S. 138, Rn. 189, Dreyer, WRP 2007, 1294, 1301, die sich diesbezüglich jedoch auf den zu diesem Zeitpunkt allein vorliegenden Referentenentwurf des BMJ vom 27.07.2007 bezog, welcher dies auf S. 41 ausdrücklich vorsah. A. A.: Bornkamm in: Köhler/Bornkamm, UWG, S 5, Rn. 1.25b, Wirring, NJW 2010, 580, $581 \mathrm{f}$. Für die Ansicht von Bornkamm und Wirring spricht, dass der Gesetzgeber die entsprechende Aussage aus dem Referentenentwurf des Bundesministeriums der Justiz vom 27.07.2007 nicht in seine Gesetzesbegründung übernommen hat, vgl. Regierungsentwurf und Begründung zum UWG vom 20.08.2008, BT.-Drs. 16/10145, S. 23.

714 Eine solche irreführende geschäftliche Handlung ist sodann nach $\ 5$ Abs. 1 Satz 1 UWG unlauter und unter den Voraussetzungen des $\ 3$ Abs. 1 UWG auch unzulässig, vgl. Kapitel 5B.I.

715 So auch Dreyer, WRP 2007, 1294, $1301 \mathrm{f}$.

716 Dies betrifft jedoch allein die Irreführung eines Verstoßes gegen einen Verhaltenskodex. Selbstverständlich kann ein solcher jedoch (zusätzlich) aus anderen Gründen unlauter sein. So bspw. aufgrund von $₫ 4$ UWG oder aufgrund der Generalklauseln in $₫ 3$ UWG.

717 Vgl. Kapitel 5B.III.1. 
sollte. ${ }^{718}$ Zudem würde eine solche Sperrwirkung auch zu nicht akzeptablen Wertungswidersprüchen zwischen geschäftlichen Handlungen im b2c-Bereich und solchen im b2b-Bereich führen, da die nach $₫ 3$ Abs. 3 UWG i. V. m Nr. $1^{719}$ und Nr. $3^{720}$ des Anhangs zu $₫ 3$ Abs. 3 UWG im b2c-Bereich stets unzulässigen Angaben in diesem Fall im b2c-Bereich nicht einmal als irreführend einzuordnen wären. Da $₫ 3$ Abs. 3 UWG für geschäftliche Handlungen im b2b-Bereich nicht anwendbar ${ }^{721}$ und ein solcher Wertungswiderspruch unbedingt zu vermeiden ist, kommt $\int 5$ Abs. 1 Satz 2 UWG bezüglich dieser Angaben im b2b-Bereich eine besondere Bedeutung zu. Denn ansonsten würde eine geschäftliche Handlung, welche im b2c-Bereich sogar stets unzulässig ist, im b2b-Bereich keine Rechtsfolgen aufgrund des UWG nach sich ziehen. Aber $₫ 5$ Abs. 1 Satz 2 UWG kommt unabhängig davon auch im Bezug auf die Angaben aus den Nr. 1 und Nr. 3 des Anhangs zu $₫ 3$ Abs. 3 UWG im b2c-Bereich eine Bedeutung zu, da $\ 5$ Abs. 1 Satz 2 UWG nicht wie diese nur unwahre Angaben erfasst, sondern auch solche, die zwar objektiv richtig, aber trotzdem zur Täuschung geeignet sind.

I. Irreführung durch unwahre Angaben aus Nr. 1 und Nr. 3 des Anhangs zu \3 Abs. 3 UWG im b2b-Bereich

\section{Grundsätzliches zur Irreführung bei unwabren Angaben}

Geschäftliche Handlungen, die unwahre Angaben über einen der in den Nr. 1 bis 7 des $\ 5$ Abs. 1 Satz 2 UWG genannten Umstände enthalten, sind nach dem allein maßgeblichen Wortlaut der Norm immer irreführend. Dies hat zunächst zur Folge, dass es bei erwiesener Unwahrheit einer Angabe unerheblich ist, ob der für die Angabe Verantwortliche sich der Unwahrheit der Angabe bewusst war beziehungsweise unter Beachtung der Erfordernisse seiner beruflichen Sorgfalt eventuell sogar davon ausgehen durfte, dass die Angabe wahr ist. ${ }^{722}$

Bezogen auf Angaben im Zusammenhang mit Verhaltenskodizes bedeutet dies, dass es für die Beurteilung einer Angabe bezüglich der Einhaltung eines Verhaltenskodexes allein darauf ankommt, ob der Verhaltenskodex eingehalten wird. Unerheblich ist dahingegen, ob der Verhaltenskodex beispielsweise von einem Subunternehmer nicht eingehalten wurde, obwohl der Verantwortliche der Angabe diesen vertraglich wirksam auf die Einhaltung des Verhaltenskodexes verpflichtet hat.

\footnotetext{
718 Dreyer, WRP 2007, 1294, 1302.

${ }^{719}$ Die Angabe, zu den Unterzeichnern eines Verhaltenskodexes zu gehören, vgl. Kapitel 5A.II.1.

${ }^{720}$ Die Angabe, ein Verhaltenskodex sei von einer öffentlichen oder einer anderen Stelle gebilligt, vgl. Kapitel 5A.II.2.

${ }_{721}$ Vgl. Kapitel 5A.

722 Urteil d. EuGH v. 19.09.2013 (CHS Tour Services GmbH / Team4 Travel GmbH). Zum Inhalt der Entscheidung siehe Fn. 538.
} 
Es bedeutet darüber hinaus, dass unwahre Angaben auch dann irreführend sind, wenn sie nicht geeignet sind, den Geschäftsverkehr zu täuschen. ${ }^{723}$ Hierfür spricht neben der Formulierung der Norm $^{724}$ auch der immer gebotene Blick auf die UGP-Richtlinie. Denn diese unterscheidet wie das UWG in ihrem Wortlaut deutlich zwischen Geschäftspraktiken, die aufgrund unwahrer Angaben irreführend sind und solchen, die irreführend sind, weil sie täuschen oder zur Täuschung geeignet sind. ${ }^{725}$ Eine entsprechende teleologische Reduktion des Wortlautes ist auch nicht notwendig, um eine unangemessene Beschränkung der unternehmerischen Handlungsfreiheit zu vermeiden. ${ }^{726}$ Denn unwahre Angaben ohne Täuschungseignung sind zwar irreführend und damit unlauter ${ }^{727}$, aber nicht zwangsläufig unzulässig. ${ }^{728}$ Denn hierfür ist wie bereits dargestellt ${ }^{729}$ notwendig, dass die geschäftliche Handlung die Interessen von Verbrauchern, Mitbewerbern oder sonstigen Marktteilnehmern spürbar beeinträchtigt. ${ }^{730}$ Eine solche spürbare Beeinträchtigung liegt jedoch dann nicht vor, wenn trotz unwahrer Angaben keine Eignung zur Täuschung vorliegt, so dass schlussendlich keine Sanktionierung der unwahren Angabe nach dem UWG erfolgt.

Dies entspricht auch der Systematik der UGP-Richtlinie ${ }^{731}$. Denn diese sieht nur dann das Verbot einer eine unwahre Angabe enthaltenden Geschäftspraktik vor, wenn die Geschäftspraktik einen Verbraucher ,in jedem Fall tatsächlich oder voraussichtlich zu einer geschäftlichen Entscheidung veranlasst, die er ansonsten nicht getroffen hätte. "732 Liegen diese beiden Voraussetzungen kumulativ 733 (unwahre Angabe und

723 A. A. Wiring, NJW 2010, 580, 581, der den Wortlaut teleologisch so reduzieren will, dass auch unwahre Angaben nur dann irreführend sind, wenn sie zur Täuschung geeignet sind.

724 Die doppelte Verwendung des Wortes „enthält" spricht dafür, dass unwahre Angaben nicht als hervorgehobener Spezialfall von zur Täuschung geeigneten Angaben einzuordnen ist, sondern eine eigene Kategorie darstellt. Denn andernfalls hätte der Gesetzgeber die Norm wie folgt formuliert: „Eine geschäftliche Handlung ist irrefübrend, wenn sie unwabre Angaben oder sonstige zur Täuschung geeignete Angaben enthält."

725 So lautet Art. 6 Abs. 1 der UGP-Richtlinie „Eine Geschäftspraxis gilt als irreführend, wenn sie falsche Angaben enthält und somit unwahr ist oder (Hervorhebung d. d. Verf.) wenn sie in irgendeiner Weise, einschließlich sämtlicher Umstände ihrer Präsentation, selbst mit sachlich richtigen Angaben den Durchschnittsverbraucher in Bezug auf einen oder mehrere der nachstehend aufgeführten Punkte täuscht oder ihn zu täuschen geeignet ist $\{\ldots\}$..“

726 So aber Wiring, NJW 2010, 580, 581.

727 \5 Abs. 1 Satz 1 UWG.

728 Regierungsentwurf und Begründung zum UWG vom 20.08.2008, BT.-Drs. 16/10145, S. 23.

${ }^{729}$ Vgl. Kapitel 5B.I.

730 \3 Abs. 1 UWG.

731 Vgl. Kapitel 2B.I.1.b.

732 Art. 6 Abs. 1 letzter HS UGP-Richtlinie.

733 Urteil d EuGH v. 19.12.2013 (Trento Sviluppo srl Centrale Adriatica Soc. coop. arl / Autorita Garante della Concorrenza e del Mercato). In diesem Urteil hat der EuGH auf die Vorlage des Consiglio di Stato (Italien) klargestellt, dass es sich bei der Veranlassung zu einer geschäftlichen Entscheidung nicht um einen eigenen Unterfall der irreführenden Geschäftspraktik handelt, sondern dass diese Tatbestandsvoraussetzung kumulativ zu den alternativen Tatbestandsvoraussetzun- 
Geeignetheit zur Veranlassung einer geschäftlichen Entscheidung) vor, ist nach den Auslegungsmaßgaben der UGP-Richtlinie immer von einer irreführenden Geschäftspraktik auszugehen. Ist jedoch eine der Voraussetzungen nicht erfüllt, ist die Geschäftspraktik bereits nicht irreführend und somit eine unwahre Angabe dann nicht irreführend, wenn sie einen Verbraucher nicht zu einer geschäftlichen Entscheidung veranlasst, die dieser ansonsten nicht getroffen hätte. Dabei muss jedoch beachtet werden, dass die Einordnung einer Geschäftspraktik als irreführend diese aufgrund der Wertungen des Art. 5 Abs. 4a) UGP-Richtlinie i. V. m. Art. 5 Abs. 1 UGP-Richtlinie in jedem Fall als verboten einstuft.

\section{Unwahre Angabe, zu den Unterzeichnern eines Verhaltenskodexes zu gehören}

Die unwahre Angabe, zu den Unterzeichnern eines Verhaltenskodexes zu gehören, welche bei geschäftlichen Handlungen gegenüber Verbrauchern nach \ 3 Abs. 3 i. V. m. Nr. 1 des Anhangs zu \3 Abs. 3 UWG stets unzulässig ist, stellt eine unternehmensbezogene Angabe dar. Denn mit der Angabe, einen Verhaltenskodex unterzeichnet zu haben, vermittelt ein Unternehmen den Eindruck, dass es sich freiwillig über die gesetzlichen Voraussetzungen hinaus zu bestimmten Verhaltensweisen verpflichtet hat. Erfolgt eine solche Angabe im Rahmen von geschäftlichen Handlungen im b2b-Verhältnis, unterfällt sie damit der Beurteilung gemäß \ 5 Abs. 1 Satz 2 Nr. 3 UWG. Dieser regelt sämtliche Angaben, welche ,die Person, Eigenschaften oder Rechte des Unternehmers" betreffen ${ }^{734}$ wie beispielsweise „Identität, Vermögen einschließlich der Rechte des geistigen Eigentums, den Umfang von Verpflichtungen, Befähigung, Status, Zulassung, Mitgliedschaften oder Beziehungen, Auszeichnungen oder Ehrungen, Beweggründe für die geschäftliche Handlung oder die Art des Vertriebs. "735

Zum besseren Verständnis können die genannten Beispiele in fünf Kategorien eingeordnet werden. ${ }^{736}$ Die erste erfasst die tatsächliche Identität des Unternehmens, die zweite die Kerndaten des Unternehmens wie Größe, Vermögensverhältnisse, Historie sowie Stellung im Markt. Die dritte betrifft die dem Unternehmen zuordbaren Schutzrechte aufgrund geistigen Eigentums, die vierte die tatsächlichen und rechtlichen Befähigungen, Qualifikationen, Mitgliedschaften und Beziehungen des Unternehmens, seines Inhabers sowie der Mitarbeiter und die fünfte Kategorie dem Unternehmen verliehene Auszeichnungen und Ehrungen. Weder die Auflistung der Beispiele noch die Einteilung in fünf Kategorien ist aber abschließend ${ }^{737}$, was im Hinblick auf die Beispiele durch die Verwendung der Formulierung ,wie“ vor der Aufzählung ausgedrückt wird. Entsprechend bietet

gen falsche und somit unwahre Angabe oder täuschende bzw. zur Täuschung geeignete Angabe vorliegen muss.

${ }^{734}$ Regierungsentwurf und Begründung zum UWG vom 20.08.2008, BT.-Drs. 16/10145, S. 24.

735 S 5 Abs. 1 Satz 2 Nr. 3 UWG.

736 Bornkamm in: Köhler/Bornkamm, UWG, \ 5, Rn. 5.2.

737 Dreyer in: Harte-Bavendamm/Henning-Bodewig, UWG, §5, E, Rn. 1. 
sich eine weite Auslegung der als Beispiele benannten Bezugspunkte an, da hierdurch die Bildung weiterer Untergruppen vermieden und damit die Übersichtlichkeit erhalten werden kann. ${ }^{738}$

Unter Beachtung dieser Kategorisierung ist die unwahre Angabe, zu den Unterzeichnern eines Verhaltenskodexes zu gehören, im Rahmen der vierten Kategorie als unwahre Angabe über die Mitgliedschaft eines Unternehmens anzusehen. Denn es stellt eine zu enge Auslegung des Begriffs der Mitgliedschaft i. S. d. I 5 Abs. 1 Satz 2 Nr. 3 UWG dar, wenn diese nur bei ,einer mit Rechten und Pflichten versehenen Stellung des Unternehmens in einem Verband" anerkannt wird. ${ }^{739}$ Auch wenn die Angabe über die Mitgliedschaft in einem Verband eine bedeutende Fallgruppe darstellt, ist eine solche Begrenzung der möglichen Mitgliedschaften weder mit dem Wortlaut der Norm, noch mit Sinn und Zweck der Einordnung einer Mitgliedschaft als unternehmensbezogene Angabe vereinbar. Letzterer ist darin zu sehen, dass die Angabe von Mitgliedschaften dazu führt, dass das Renommee, die Seriosität beziehungsweise die Reputation der jeweiligen Institution auf das Mitglied abstrahlt und der Verkehr aus ihr eine Vernetzung und Anerkennung des Unternehmens mit und durch andere entnimmt. ${ }^{740}$ Dies gilt jedoch nicht nur für die Mitgliedschaft in Verbänden, sondern beispielsweise auch für solche in Institutionen aus dem kulturellen, sozialen oder wissenschaftlichen Bereich. ${ }^{741}$ So profitieren beispielsweise die Teilnehmer des UN Global Compact ${ }^{742}$ in erheblichem Maße von dem Renommee der Vereinten Nationen sowie dem aufgrund ihrer Teilnahme erweckten Eindruck einer internationalen Bedeutung, auch wenn es sich bei dem UN Global Compact weder um einen Verband handelt noch um eine ,formal membership organization". ${ }^{743}$ Aber es bedarf nicht zwangsläufig eines großen Namens, um von einer Mitgliedschaft zu profitieren. Vielmehr reicht es bereits aus, dass aus der Mitgliedschaft positive Rückschlüsse auf die Mitglieder gezogen werden. Dies trifft jedoch auch auf Verhaltenskodizes zu, da diese ja als Wesenskern enthalten, dass sich die durch ihn Verpflichteten über die gesetzlichen Anforderungen hinaus den Werten der Gesellschaft entsprechend engagieren ${ }^{744}$ und „vielfach bereits ihre pure Existenz als positiver Hinweis auf eine positive Ausgestaltung

\footnotetext{
738 Vgl. Fn. 737.

739 A. A.: Dreyer in: Harte-Bavendamm/Henning-Bodewig, UWG, \ 5, E, Rn. 172.

740 Pfeifer in: Fezer, UWG, \5, Rn. 402.

${ }^{741}$ Beispielsweise aufgrund einer Fördermitgliedschaft für Oper, Theater, Ballett oder sonstige kulturelle Einrichtungen.

742 Vgl. Kapitel 1C.III.2.

743 Antwort auf die FAQ Nr. 7 auf www.unglobalcompact.com , wobei diese Aussage im Bezug darauf erfolgt, dass keine Mitgliedsgebühren (, ,any financial obligations") erhoben werden, abrufbar unter http://www.unglobalcompact.org/AboutTheGC/faq.html, zuletzt abgerufen am 19.04.2013.

744 Vgl. Kapitel 1A und Kapitel 3.
} 
der Unternehmenspolitik gewertet wird ${ }^{\star} .745$ Zudem erscheint es auch zu weitgehend, eine Mitgliedschaft i. S. d. \5 Abs. 1 Satz 2 Nr. 3 UWG nur anzuerkennen, wenn sich aus dieser tatsächlich Rechte und Pflichten ergeben. ${ }^{746}$ Denn bei den mit der Mitgliedschaft in einem Verband verbundenen Rechten und Pflichten handelt es sich oftmals nur um die Pflicht zur Leistung eines Mitgliedsbeitrages und der allgemeingehaltenen Unterstützung der Verbandsinteressen beziehungsweise um das Recht der Teilnahme an Verbandsveranstaltungen, der Nutzung von Verbandseinrichtungen und Zeichen sowie der Teilnahme an der verbandsinternen Willensbildung. Ob solche Rechte und Pflichten bestehen, hat aber keinen Einfluss auf das, was der Verkehr der Angabe über eine Mitgliedschaft entnimmt. Selbst wenn bestimmte Verhaltenskodizes wie insbesondere unilaterale Erklärungen von Unternehmen keine Mitgliedschaft i. S. d. \5 Abs. 1 Satz 2 Nr. 3 UWG darstellen sollten, stellt die Angabe, zu ihren Unterzeichnern zu gehören, trotzdem eine unternehmensbezogene Angabe i. S. d. Norm dar. Denn in jedem Fall bestehen die dargestellten erheblichen Übereinstimmungen, so dass aufgrund der Vergleichbarkeit der Sachverhalte eine unterschiedliche rechtliche Behandlung nicht gerechtfertigt wäre.

Dahingegen stellt die alleinstehende Angabe eines Unternehmens, zu den Unterzeichnern eines Verhaltenskodexes zu gehören, keine Angabe über ein wesentliches Merkmal einer Ware oder Dienstleistung i.S. d. $\$ 5$ Abs. 1 Satz 2 Nr. 1 UWG dar. Denn eine solche Angabe ist primär als unternehmensbezogene Angabe einzuordnen und lässt keine direkten Rückschlüsse auf bestimmte Merkmale der Waren und Dienstleistungen des Unternehmens zu. Grund hierfür ist die Freiheit der Unternehmen bei der inhaltlichen Ausgestaltung ihrer Verhaltenskodizes sowie die unterschiedlichen Ausrichtungen der Verhaltenskodizes. So dürfte zwar jeder Verhaltenskodex aus dem Bereich der sozialen Mindeststandards einen Ausschluss von Kinderarbeit enthalten, während ein solcher aufgrund des fehlenden Sachzusammenhangs in Verhaltenskodizes aus dem Umweltbereich im Regelfall nicht enthalten sein dürfte. Gleichfalls wird der Durchschnittverbraucher der Angabe, dass ein Unternehmen einen Verhaltenskodex unterzeichnet hat, keine konkrete Verpflichtung auf eine bestimmte Verhaltensweise entnehmen, sondern lediglich ein grundsätzliches Bekenntnis des Unternehmens zu einem verantwortungsvollen und den Werten der Gesellschaft entsprechenden Verhalten. Hierunter versteht der Durchschnittsverbraucher jedoch wiederum eine breite Palette, welche von sozialem Engagement über ein gutes Preis-Leistungsverhältnis bis hin zu Umweltschutzmaßnahmen reicht. ${ }^{747}$ Dabei hat eine Untersuchung des Instituts

745 IMUG Arbeitspapier 17/2007, Corporate Social Responsibility als Verbraucherinformation, S. 25.

746 A. A.: Dreyer in: Harte-Bavendamm/Henning-Bodewig, UWG, \5, E, Rn. 172.

${ }^{747}$ Ergebnisse der repräsentativen Haushaltsbefragung „CSR-Informationsbedarf von Verbrauchern Informations- und Einkaufsverbalten im Kontext von CSR" des imug, S. 9, abrufbar unter 
für Markt-Umwelt-Gesellschaft e. V. der Universität Hannover gezeigt, dass die meisten Verbraucher immer nur wenige Verhaltensweisen aus den vorbenannten Handlungsfeldern mit verantwortungsvollem Unternehmenshandeln assoziieren. ${ }^{748}$ Notwendig für die Annahme einer irreführenden Angabe über ein wesentliches Merkmal einer Ware oder Dienstleistung i. S. d. \5 Abs. 1 Satz 2 Nr. 1 UWG ist bei Angaben im Zusammenhang mit Verhaltenskodizes somit immer, dass auf konkrete Inhalte des Verhaltenskodexes Bezug genommen wird. ${ }^{749}$ In diesen Fällen liegt eine mögliche Irreführung bei einem der Angabe widersprechenden Verhalten aber nicht in der Angabe, dass ein entsprechender Verhaltenskodex unterzeichnet wurde, sondern in der unwahren Abgabe über die Einhaltung der ausdrücklich benannten Verhaltensweisen selbst ${ }^{750}$ und, wenn die Voraussetzungen des $\int 5$ Abs. 1 Satz 2 Nr. 6 UWG erfüllt sind ${ }^{751}$, zusätzlich in dem Verstoß gegen den Verhaltenskodex.

\section{Unwabre Angabe über Billigung eines Verhaltenskodex}

Die unwahre Angabe, ein Verhaltenskodex sei von einer öffentlichen oder einer anderen Stelle gebilligt, welche bei geschäftlichen Handlungen gegenüber Verbrauchern nach $\int 3$ Abs. 3 i. V. m. Nr. 3 des Anhangs zu \3 Abs. 3 UWG stets unzulässig ist, stellt wie die Angabe, zu den Unterzeichnern eines Verhaltenskodexes zu gehören, eine unternehmensbezogene Angabe dar und unterfällt damit im b2b-Verhältnis der Beurteilung nach $\int 5$ Abs. 1 Satz 2 Nr. 3 UWG. Dabei ist es unschädlich, dass der Begriff der Billigung in dessen Auflistung nicht ausdrücklich benannt wird, da die Billigung unter den in der Auflistung genannten Begriff der Auszeichnung gefasst werden kann. Dieser ist weit auszulegen, da die Auszeichnung selbst auch nur ein Beispiel für eine unternehmensbezogene Angabe ist ${ }^{752}$ und alles umfasst, was ein Unternehmen aus der Menge der Mitbewerber hervorhebt und von dritter Seite verliehen wurde. ${ }^{753}$ Hintergrund dieser weiten Auslegung ist die Tatsache, dass Auszeichnungen nach allgemeiner Meinung ein beson-

http://www.imug.de/pdfs/verbraucher/hp_imug_CSR_Verbraucher_Repraesentativbefragung _2006_03_16.pdf, zuletzt abgerufen am 29.04.2013.

748 Imug Arbeitspapier 17/2007, Corporate Social Responisibility als Verbraucherinformation, S. 9.

749 So beispielsweise durch eine Aussage wie „Unser Spezialkaffee wird auf Grundlage unseres Verhaltenskodexes hergestellt, welcher ein Verbot der Kinderarbeit sowie einen Verzicht auf den Einsatz, von Pflanzenschutzmitteln vorsieht".

750 So müsste bei der in Fn. 749 als Beispiel genannten Aussage geprüft werden, ob es sich bei dem Verzicht auf Kinderarbeit beziehungsweise auf den Einsatz von Pflanzenschutzmittel um wesentliche Merkmale der Ware Kaffee i. S. d. \5 Abs. 1 Satz 2 Nr. 1 UWG handelt.

751 Vgl. zu den Voraussetzung einer Irreführung nach \5 Abs. 1 Satz 2 Nr. 6 UWG Kapitel 5B.

752 Weidert in: Harte-Bavendamm/Henning-Bodewig, UWG, \5, E, Rn. 176, vgl. zur grundsätzlich gebotenen weiten Auslegung der Beispiele in $₫ 5$ Abs. 1 Satz 2 Nr. 3 UWG auch 0.

753 Bornkamm in: Köhler/Bornkamm, UWG, \5, Rn. 5.158, Weidert in: HarteBavendamm/Henning-Bodewig, UWG, $\ 5$, E, Rn. 176, der jedoch das Merkmal der Verleihung durch Dritte nicht explizit erwähnt. 
derer Werbewert und somit ein besonderes Missbrauchspotenzial zugesprochen wird. ${ }^{754}$ Dies gilt insbesondere dann, wenn der Empfänger der geschäftlichen Handlung zum Zeitpunkt der Entscheidung die Qualität der Ware oder Dienstleistung nicht beurteilen kann und sich daher auf die Eigenschaften des anbietenden Unternehmers fokussieren muss. Entsprechend sind beispielsweise Empfehlungen von Dritten, Zertifizierungen, behördliche Anerkennungen, Medaillen, Urkunden als Auszeichnungen i. S. d. \5 Abs. 1 Satz 2 Nr. 3 UWG anerkannt. ${ }^{755}$ Wie mit diesen verbindet der Verbraucher mit der Billigung des Verhaltenskodexes eines Unternehmens durch eine öffentliche oder eine andere Stelle eine Hervorhebung gegenüber anderen Unternehmen.

II. Irreführung trotz objektiver Richtigkeit der Angaben aus Nr. 1 und Nr. 3 des Anhangs zu $₫ 3$ Abs. 3 UWG

\5 Abs. 1 Satz 2 UWG beschränkt sich in richtlinienkonformer Art und Weise anders als Nr. 1 und Nr. 3 des Anhangs zu $\ 3$ Abs. 2 UWG nicht auf den Schutz der Entscheidungsfreiheit durch unwahre Angaben, sondern umfasst auch objektiv richtige Angaben, soweit sie trotz ihrer objektiven Richtigkeit zur Täuschung geeignet sind. ${ }^{756}$ Es ist somit nach dem Wortlaut der Norm keine Voraussetzung, dass die objektiv richtige Angabe tatsächlich zu einer Täuschung im Geschäftsverkehr führt. Ausreichend ist vielmehr die grundsätzliche Eignung hierzu. ${ }^{757}$ Entsprechend kann auch eine geschäftliche Handlung, welche die objektiv richtige Angabe, dass ein Unternehmer zu den Unterzeichnern eines Verhaltenskodizes gehört ${ }^{758}$ oder das ein Verhaltenskodex durch eine öffentliche oder eine andere Stelle gebilligt wurde ${ }^{759}$, irreführend sein, wenn sie geeignet ist, den mit ihr angesprochenen Verkehrskreis zu täuschen. Anders als die Irreführung nach $₫ 5$ Abs. 1 Satz 2 UWG aufgrund unwahrer Angaben 760 ist die Irreführung aufgrund einer Täuschungseignung trotz objektiver Richtigkeit der Angabe somit bei den Angaben aus den Nummern 1 und 3 des Anhangs zu $\ 3$ Abs. 3 UWG auch für den b2c-Bereich von Bedeutung.

$\mathrm{Da}$ es sich bei dem Tatbestandsmerkmal der Täuschungseignung einer objektiv richtigen Angabe um ein in besonderem Maße subjektives Tatbestandsmerk-

754 Peifer in: Fezer, UWG. \5, Rn. 400.

755 Peifer in: Fezer, UWG. \5, Rn. 400 ff., Weidert in: Harte-Bavendamm/Henning-Bodewig, UWG, $\int 5$, E, Rn. $176 \mathrm{ff}$.

756 Die UGP-Richtlinie schützt die Entscheidungsfreiheit auch vor sachlich richtigen Angaben, wenn durch diese eine Täuschung erfolgt oder sie zur Täuschung geeignet ist, Art. 6 Abs. 1 UGPRichtlinie. Entsprechend muss in diesem Punkt keine Unterscheidung zwischen geschäftlichen Handlungen im b2b-Bereich und solchen im b2c-Bereich vorgenommen werden.

${ }_{757}$ Bornkamm in: Köhler/Bornkamm, UWG, \ 5, Rn. 2.65.

758 Zur Auslegung des Umfangs dieser Angabe vgl. Kapitel 5A.II.1.

759 Zur Auslegung des Umfangs dieser Angabe vgl. Kapitel 5A.II.2.

${ }^{760}$ Vgl. Kapitel 6B.I. 
mal handelt, muss bei seiner Ermittlung darauf abgestellt werden, an wen sich die Angabe und damit die geschäftliche Handlung richtet. ${ }^{761}$ Denn ein und dieselbe Angabe kann von verschiedenen Verkehrskreisen (beispielsweise einerseits Fachleute, andererseits Durchschnittsverbraucher) extrem unterschiedlich verstanden werden. Entsprechend richtet sich die Täuschungseignung einer Angabe nach dem Verständnis der angesprochenen Verkehrskreise. ${ }^{762}$ Im Rahmen von geschäftlichen Handlungen im Zusammenhang mit Verhaltenskodizes wird es sich dabei zwar zumeist um die Allgemeinheit handeln, jedoch erfolgen Angaben zu Verhaltenskodizes selbstverständlich auch im Rahmen von geschäftlichen Handlungen gegenüber bestimmten Verkehrskreisen.

\section{Irreführung trotz tatsächlicher Billigung eines Verhaltenskodexes durch eine öffentliche oder eine andere Stelle}

Im Hinblick auf die Angabe, dass ein Verhaltenskodex von einer öffentlichen oder einer anderen Stelle gebilligt wurde, kann eine Irreführung nach $\int 5$ Abs. 1 Satz 2 Nr. 3 UWG trotz tatsächlich vorliegender entsprechender Billigung gegeben sein. Voraussetzung einer Irreführung ist in diesem Fall jedoch, dass die objektiv richtige Angabe geeignet ist, ihren Empfänger zu täuschen. Eine gerichtliche Entscheidung darüber, wann die objektiv richtige Angabe der Billigung eines Verhaltenskodexes eine solche Täuschungseignung aufweist, liegt bisher nicht vor. Das LG Darmstadt hat zwar über einen Sachverhalt entschieden, der auch als Billigung eines Verhaltenskodexes angesehen werden könnte, hat diesen jedoch allein unter dem Gesichtspunkt der irreführenden Verwendung eines Gütezeichens beurteilt. ${ }^{763}$ Gegenstand dieses Verfahrens vor dem LG Darmstadt war die Verwendung des Gütesiegels ${ }^{764}$ des BVDVA ${ }^{765}$. Dieses Gütesiegel erteilte der BVDVA behördlich zugelassene Versandapotheken ${ }^{766}$, die eine vorformulierte Selbstverpflichtung unterschrieben hatten und vom BVDVA überprüft worden waren. ${ }^{767}$ Trotz der alleinigen Einordnung als Gütezeichen bleiben die Ausführungen des LG Darmstadt bereits deshalb von Belang für die hier vorzunehmende Bewertung, da das LG Darmstadt den Sachverhalt nicht gemäß $\int 5$ Abs. 2 Nr. 1

\footnotetext{
${ }^{761}$ Bornkamm in: Köhler/Bornkamm, \5, Rn. 2.75 f.

762 Vgl. Fn. 761.

763 Urteil des LG Darmstadt v. 22.11.2008 - 22 O 100/08 („BVDVA-geprüft“), redigierte Leitsätze und Gründe in MMR 2009, 277, 278 f.

${ }^{764}$ Eigenbezeichnung, vgl Pressemitteilung des BVDVA vom 16.05.2007 auf http://www.presseportal.de/pm/43693/987327/guetesiegel-schuetzt-vor-arzneifaelschungenarzneiversandhandel-guetesiegel-sorgt-fuer-sicherheit, zuletzt abgerufen am 12.06.2013.

765 Bundesverband Deutscher Versandapotheken.

766 Und somit an seine potenziellen Mitglieder.

${ }^{767} \mathrm{Zu}$ den Anforderungen vgl. die Pressemitteilung des BVDVA vom 16.05.2007 auf http://www.presseportal.de/pm/43693/987327/guetesiegel-schuetzt-vor-arzneifaelschungenarzneiversandhandel-guetesiegel-sorgt-fuer-sicherheit, zuletzt abgerufen am 12.06.2013.
} 
UWG 2004 als Angabe über die Merkmale einer Ware oder Dienstleistung, sondern gemäß $\ 5$ Abs. 2 Nr. 1 UWG 2004 als Auszeichnung eines Unternehmens und damit als unternehmensbezogene Angabe behandelt hat. ${ }^{768}$

Unabhängig davon sind die Rechtsausführungen im Hinblick auf Gütezeichen auch für die Beurteilung von Angaben über die Billigung eines Verhaltenskodexes immer von Bedeutung, da zwischen beiden eine grundsätzliche Vergleichbarkeit besteht. Denn wie die Billigung eines Verhaltenskodexes im Speziellen und die Auszeichnung i. S. d. $\ 5$ Abs. 1 Satz 2 Nr. 3 UWG im Allgemeinen ${ }^{769}$ versteht der Verkehr Gütezeichen als Kennzeichen dafür, dass eine Überprüfung durch einen neutralen Dritten mit entsprechender Kompetenz nach objektiven Kriterien durchgeführt wurde. ${ }^{770}$ Dabei ist es für die Vergleichbarkeit unschädlich, dass Prüf- und Gütezeichen im Regelfall Waren- und Dienstleistungen betreffen ${ }^{771}$, während sich Auszeichnungen auf das dahinterstehende Unternehmen beziehen. Dies gilt insbesondere, da sich mit guten Argumenten vertreten lässt, Gütesiegel ebenfalls als Auszeichnung i. S. d. \5 Abs. 1 Satz 2 Nr. 3 UWG einzuordnen. 772

Für Gütezeichen hat der BGH ${ }^{773}$ schon unter Geltung des UWG 1909 festgestellt, dass allein der Umstand, dass eine Prüfbescheinigung ${ }^{774}$ ergangen ist und der Hinweis darauf insoweit richtig war, die Annahme einer irreführenden Werbung nicht ausschließt. ${ }^{775}$ Dabei war es für den BGH sogar unerheblich, dass die Prüfbescheinigung einen Verwaltungsakt darstellte, welchem selbst bei Rechtswidrigkeit ein besonderer Vertrauensschutz zukommt. ${ }^{776}$ Zur Begründung führte der

${ }^{768}$ Urteil des LG Darmstadt v. 22.11.2008 - 22 O 100/08 („BVDVA-geprüft“) in MMR 2009, $277 \mathrm{ff}$.

${ }^{769}$ Vgl. Kapitel 6B.I.3.

770 Weidert in: Harte-Bavendamm/Henning-Bodewig, UWG, \5, C, Rn. 260, Baldauf, S. 4.

${ }^{771}$ Für einen strengen Bezug auf Waren und Dienstleistungen Peifer in: Fezer, UWG, \5, Rn. 400, A. A. Baldauf, S. 4, die auch unternehmensbezogene Zeichen als Gütezeichen zulässt, den Begriff des Gütezeichens im Rahmen ihrer Definition dann aber auf Zeichen beschränkt, die anzeigen, dass eine Ware oder Dienstleistung von einem neutralen Dritten mit entsprechender Kompetenz und Objektivität beurteilt wurde. Damit verbleiben als unternehmensbezogene Gütezeichen nur solche, die sämtliche Produkte eines Unternehmens betreffen.

772 Urteil des LG Darmstadt v. 22.11.2008 - 22 O 100/08 („BVDVA-geprüft“) in MMR 2009, 277 ff., zustimmend Blasek, MMR 2009, 279, Baldauf, S. 70, Weidert in: HarteBavendamm/Henning-Bodewig, UWG, \5, E, Rn. 178 unter Verweis auf Urteil des BGH v. 04.10.1990 - I ZR 39/39 (OLG Köln) (Zaunlasur) in GRUR 1991, 550, 551.

${ }^{773}$ Urteil des BGH v. 23.10.1997 - I ZR 98/95 (OLG Frankfurt a. M.) (GS-Zeichen) in GRUR 1998, 1043, 1044.

${ }^{774}$ Zur synonymen Verwendung der Begrifflichkeiten Gütezeichen, Prüfzeichen, Prüfbescheinigung etc., Baldauf, S. 4.

775 Die Bezugnahme des BGH auf eine irreführende Werbung anstatt auf eine irreführende geschäftliche Handlung ergibt sich aus dem zum Zeitpunkt der Entscheidung entsprechend beschränkten Anwendungsbereich des UWG (vgl. hierzu Kapitel 4A). Der Grundsatz der Irreführung durch objektiv richtige Angaben ist jedoch hiervon nicht betroffen.

$776 \int 48$ Abs. 2 VwVfG 
BGH an, dass das Vorliegen einer entsprechenden Prüfbescheinigung nicht von der grundsätzlichen Verpflichtung befreie, irreführende Angaben zu unterlassen. ${ }^{777}$ Voraussetzung für eine Irreführung war demnach (wie heute durch $\int 5$ Abs. 1 Satz 2 UWG i. V. m. \3 Abs. 1 UWG kodifiziert), dass ,der Verkehr, für den sie (die objektiv richtige Angabe, Ergänzung d. d. Verf.) bestimmt ist, ibr etwas Unrichtiges entnimmt und die dadurch geweckte Feblvorstellung geeignet ist, das Kaufverhalten eines nicht unbeachtlichen Teils des Verkehrs zu beeinflussen." 778

a. Irreführung wegen Fehlens der Voraussetzungen der Billigung

Wenn eine öffentliche oder eine andere Stelle einen Verhaltenskodex billigt, obwohl die Anforderungen, die sie selbst an eine Billigung stellt, nicht vorliegen und damit eine Billigung eigentlich nicht hätte erfolgen dürfen, unterfällt eine Angabe über die erfolgte Billigung des Verhaltenskodex nicht dem Anwendungsbereich der Nr. 3 des Anhangs zu $\int 3$ Abs. 3 UWG. Denn dieser erfasst nur die unwahre Angabe über das Vorliegen einer entsprechenden Billigung. ${ }^{779}$ Dahingegen erfasst \5 Abs. 1 Satz 2 UWG auch wahre Angabe, die geeignet sind, den Verkehr zu täuschen. Diese Täuschungseignung besteht im Hinblick auf Billigungen, die aufgrund des Fehlens der Voraussetzungen der Billigung nicht hätten erteilt werden dürfen, darin, dass Verkehr gerade glaubt, dass die Verhaltenskodizes diese Voraussetzungen erfüllen.

Für die Verwendung eines tatsächlich verteilten Gütezeichens ist anerkannt, dass eine zur Täuschung geeignete Angabe vorliegt, wenn die der Erteilung zugrundeliegende Prüfung in entscheidenden Punkten unvollständig war und das Gütezeichen nicht hätte verliehen werden dürfen. ${ }^{780}$ Dabei kommt es nach Ansicht des BGH nicht darauf an, ob die betroffenen Verkehrskreise wissen, welche Voraussetzungen für die Erteilung des Gütezeichens vorliegen müssen. ${ }^{781}$ Denn auch ohne dieses Detailwissen gehen diese davon aus, dass das Produkt die Voraussetzungen, die das Gütesiegel erfordert, vorweist und keine Mängel aufweist, die zur Rücknahme des Siegels führen müssten. ${ }^{782}$ Ebenfalls unerheblich ist, ob der die Angabe tätigende Unternehmer Kenntnis oder zumindest schuldhafte Unkenntnis von der Tatsache haben muss, dass seine Ware oder Dienstleistung die Voraussetzung des Gütezeichens nicht erfüllt. ${ }^{783}$

\footnotetext{
777 Vgl. Fn. 773.

778 Vgl. Fn. 773.

${ }_{779}$ Vgl. Kapitel 5A.II.2.

${ }^{780}$ Vgl. Fn. 773, entsprechend auch Wiebe, WRP 1993, 71, 80 f., Baldauf, S. 67 f.

781 Vgl. Fn. 773, entsprechend auch Wiebe, WRP 1993, 71, 80 f., Baldauf, S. 67 f.

782 Vgl. Fn. 773, entsprechend auch Wiebe, WRP 1993, 71, 80 f., Baldauf, S. 67 f.

783 Baldauf, S. 67 f., die zu Recht auf die Verschuldensunabhängigkeit des Unterlassungsanspruchs verweist.
} 
Überträgt man diese Maßgaben auf die erfolgte Billigung eines Verhaltenskodexes durch eine öffentliche oder eine andere Stelle, liegt eine Irreführung vor, wenn die Voraussetzungen, an welche die billigende Stelle die Billigung geknüpft hat, tatsächlich bei Erteilung der Billigung nicht vorlagen oder zu einem späteren Zeitpunkt weggefallen sind. ${ }^{784}$ So beispielsweise im Hinblick auf die in Deutschland gesetzlich vorgesehene Billigung eines Verhaltenskodexes beziehungsweise Anerkennung einer Wettbewerbsregel nach $\ 24$ Abs. 3 ff. GWB durch das Bundeskartellamt ${ }^{785}$, obwohl die Voraussetzungen für diese Billigung beziehungsweise Anerkennung nicht vorlagen. Eine Übertragung dieser Maßgaben ist angemessen, da das Vertrauen des Verkehrs und damit das Täuschungspotenzial bei diesen Sachverhaltsvarianten vergleichbar ist. Denn sowohl bei der Billigung eines Verhaltenskodexes als auch bei der Erteilung eines Prüf- oder Gütezeichens für eine Ware oder Dienstleistung geht der Verkehr davon aus, dass diese an bestimmte Voraussetzungen geknüpft sind und diese Voraussetzungen von dem Verhaltenskodex beziehungsweise der Ware oder Dienstleistung erfüllt werden. Ist dies nicht der Fall, ist eine entsprechende Angabe unabhängig davon, ob der Verkehr die konkreten Voraussetzungen der Billigung beziehungsweise der Verleihung des Prüf- oder Gütezeichens kennt, geeignet, den Verkehr zu täuschen. Gleiches gilt auch für die Sachverhaltsalternativen, in welchen die Voraussetzungen für die Billigung zwar zum Zeitpunkt der Billigung vorlagen, jedoch zu einem späteren Zeitpunkt weggefallen sind beziehungsweise die Anforderungen der erteilten Billigung verändert wurden. Denn in diesen Fällen liegt eine identische Täuschungseignung vor, da der Verhaltenskodex gleichfalls nicht die Voraussetzungen erfüllt, die der Verkehr aufgrund der angegebenen Billigung ${ }^{786}$ voraussetzt. ${ }^{787}$

\section{b. Irreführung aufgrund fehlender Neutralität der billigenden Stelle}

Gibt ein Unternehmer in einer geschäftlichen Handlung an, ein Verhaltenskodex sei von einer öffentlichen oder einer anderen Stelle gebilligt, entnimmt der Geschäftsverkehr dieser Aussage, dass die billigende Stelle neutral ist. Für Gütezeichen wurde die dort auch vorausgesetzte Neutralität der verleihenden Stelle beispielsweise verneint, wenn die Verleihung des Gütezeichens erkauft wurde. ${ }^{788}$ Ein solches Erkaufen eines Gütezeichens liegt jedoch nicht automatisch vor, sobald Zahlungen von dem das Gütezeichen Erhaltenden an den Verleihenden geleistet

\footnotetext{
${ }^{784}$ Entscheidend kann nur sein, ob die Voraussetzungen der Billigung zum Zeitpunkt der Angabe der Billigung vorlagen oder nicht, den nur dieser Zeitpunkt ist für die Beurteilung der Täuschungshandlung relevant. Entsprechend auch für eine Irreführung bei einer nachträglichen Veränderung der Merkmale eines Gütezeichens, Wiebe, WRP 1993, 71, 80.

785 Vgl. zum Begriff der Wettbewerbsregel i. S. d. \$24 GWB Kapitel 3F.III.

${ }^{786}$ Nämlich das Erfüllen der Anforderungen der Billigung.

${ }^{787}$ Entsprechend für diese Sachverhaltsalternativen bei Gütezeichen, Baldauf, S. 68.

788 Beschluss des OLG Frankfurt a. M. v. 08.03.1994 - 6 W 16/94 (Touristik-Gütesiegel) in NJWRR 1994, 523, Baldauf, S. 65.
} 
werden. ${ }^{789}$ Voraussetzung für die Zulässigkeit solcher Zahlungen ist jedoch, dass die Zahlungen einer konkreten Leistung des Verleihenden zugeordnet werden können, die unabhängig von der Frage der Verleihung selbst ist. So schließt eine Gebühr, durch welche die Überprüfung und Kontrolle finanziert wird, die Neutralität der verleihenden Stelle nicht aus, soweit diese angemessen ist und einheitlich erhoben wird. ${ }^{790}$ Nicht zulässig ist dahingegen eine jährlich zu leistende Lizenzgebühr für die Nutzung des Gütezeichens sowie die Zahlung eines jährlichen Mitgliedsbeitrages in der Gütezeichengemeinschaft. ${ }^{791} \mathrm{Zu}$ weit geht es aber, wenn im Hinblick auf die Annahme der Neutralität gefordert wird, dass die verleihende Stelle aufgrund eines Anerkennungsverfahrens zur Erteilung des Gütesiegels berechtigt ist. ${ }^{792}$ Denn dies wird vom Geschäftsverkehr nicht erwartet. Gleichfalls spricht es nicht grundsätzlich gegen die Neutralität im Hinblick auf die Bewertung eines Gütesiegels, wenn es sich bei der bewertenden Stelle um den Interessensverband derjenigen handelt, denen das Gütezeichen verliehen wird. ${ }^{793}$ Auch diese Auslegungsmaßgaben zur notwendigen Neutralität können auf die den Verhaltenskodex billigende Stelle übertragen werden. Denn der Geschäftsverkehr geht davon aus, dass die Billigung des Verhaltenskodexes ohne wirtschaftliche Gegenleistung erfolgt.

c. Irreführung aufgrund der Bezeichnung der billigenden Stelle

Unabhängig davon, ob es sich um die Billigung eines Verhaltenskodexes oder die Verleihung eines Gütezeichens handelt, ist eine entsprechende Angabe dann zur Täuschung des Geschäftsverkehrs geeignet, wenn dieser aufgrund der Bezeichnung der billigenden oder verleihenden Stelle einen falschen Eindruck von deren Bedeutung oder Qualifizierung bekommt. So beispielsweise dann, wenn der Verkehr aufgrund der Bezeichnung der billigenden Stelle den Eindruck gewinnen kann, es handele sich um eine Körperschaft des öffentlichen Rechts oder zumindest um eine Institution, die von einer solchen Körperschaft beaufsichtigt oder

\footnotetext{
${ }^{789}$ Beschluss des OLG Frankfurt a. M. v. 08.03.1994 - 6 W 16/94 (Touristik-Gütesiegel) in NJWRR 1994, 523.

790 Urteil des BGH v. 04.10.1990 - I ZR 39/39 (OLG Köln) (Zaunlasur) in NJW 1991, 1229, 1230, Baldauf, S. 65, die diesbezüglich einen Vergleich zur Werbung mit Testergebnissen zieht, wo eine Finanzierung des Test durch die Getesteten ebenfalls gestattet ist.

${ }^{791}$ Beschluss des OLG Frankfurt a. M. v. 08.03 .1994 - 6 W 16/94 (Touristik-Gütesiegel) in NJWRR 1994, 523, Urteil des LG Darmstadt v. 22.11.2008 - 22 O 100/08 („BVDVA-geprüft“) in MMR 2009, 277, 279. A. A. Baldauf, S. 65.

792 Weidert, GRURPrax 2010, 351, 353, A. A. Urteil des LG Darmstadt v. 22.11.2008 - 22 O 100/08 (,BVDVA-geprüft“) in MMR 2009, 277, 278 f.

793 Blasek, Anmerkungen zum Urteil des LG Darmstadt v. 22.11.2008 - 22 O 100/08 („BVDVAgeprüft") in MMR 2009, 279, 280, A. A. Urteil des LG Darmstadt v. 22.11.2008 - 22 O 100/08 (,BVDVA-geprüft“) in MMR 2009, 277, 278 f.
} 
gefördert wird. ${ }^{794}$ Ausreichend ist aber auch, dass sich ein Verband als Verbraucherverband bezeichnet, seine Mitglieder aber zu einem erheblichen Anteil Unternehmen sind oder dass sich ein Verband als Bundesverband bezeichnet, obwohl er nicht bundesweit tätig ist. ${ }^{795}$

\section{Irrefübrung trotz tatsäcblicher Unterzeichnung eines Verbaltenskodexes}

Weniger umfangreich sind die Möglichkeiten einer Irreführung gemäß $\ 5$ Abs. 1 Satz 2 Nr. 3 UWG trotz der objektiv richtigen Angabe, zu den Unterzeichnern eines Verhaltenskodexes zu gehören. ${ }^{796}$ Diese Angabe versteht der Geschäftsverkehr dahingehend, dass der Unternehmer den Verhaltenskodex nicht nur unterzeichnet hat, sondern durch seine Unterzeichnung auch an diesen gebunden ist. Eine Täuschungseignung liegt somit nur dann vor, wenn der Verhaltenskodex zwar unterzeichnet wurde, die Unterzeichnung oder der Verhaltenskodex selbst tatsächlich aber unwirksam ist. ${ }^{797}$ Dahingegen erwartet der Geschäftsverkehr aufgrund der Angabe, zu den Unterzeichnern eines Verhaltenskodexes zu gehören, nicht, dass sich der Unternehmer beziehungsweise das Unternehmen durch den Verhaltenskodex in justiziabler Art und Weise verpflichtet hat. Denn die rechtliche Unverbindlichkeit von Verhaltenskodizes ist Teil des Verständnisses des Begriffs im allgemeinen Sprachgebrauch. Aus diesem Grund stellt es keine Irreführung dar, wenn der Unterzeichner einer Absichtserklärung ${ }^{798}$ angibt, einen Verhaltenskodex unterzeichnet zu haben.

III. Irreführung durch weitere Angaben im Zusammenhang mit Verhaltenskodizes

Selbstverständlich sind nicht nur die Angaben aus den Nr. 1799 und Nr. 3800 des Anhangs zu $₫ 3$ Abs. 3 UWG an den Vorgaben von $\ 5$ Abs. 1 Satz 2 UWG zu messen, sondern sämtliche im Rahmen von geschäftlichen Handlungen im b2bund b2c-Bereich getätigten Angaben im Zusammenhang mit Verhaltenskodizes. Täuschungsgeeignet sind hierbei sämtliche Angaben über den Inhalt eines Verhaltenskodexes sowie über die Überprüfung seiner Einhaltung. Betreffen diese Angaben einen Umstand aus $₫ 5$ Abs. 1 Satz 2 UWG und sind unwahr, ist die zu-

\footnotetext{
${ }^{794}$ Urteil des OLG Dresden v. 29.02.2000 - 14 U 3716/99, Leitsätze in WRP 2010, 1202 betreffend der Werbung mit dem Gütesiegel einer „Europäischen Wirtschaftskammer“.

795 Urteil des OLG Hamm v. 10.10.2002 - 4 U 64/02 in BeckRS 2010, 09440 ff.

${ }^{796} \mathrm{Zu}$ den von dieser Angabe erfassten Sachverhaltsalternativen vgl. Kapitel 5A.II.1.

${ }^{797}$ Zur Frage, ob in diesem Fall eine entsprechende Angabe nicht bereits unwahr ist vgl. Kapitel 5A.II.1.

798 Zur Bedeutung des Begriffs vgl. Kapitel 3D.

${ }^{799}$ Die Angabe, zu den Unterzeichnern eines Verhaltenskodexes zu gehören.

800 Die Angabe, ein Verhaltenskodex sei von einer öffentlichen oder einer anderen Stelle gebilligt.
} 
grundeliegende geschäftliche Handlung irreführend. ${ }^{801}$ Gleiches gilt auch, wenn die Angabe zwar wahr ist, aber trotzdem zur Täuschung geeignet ist. 802

\section{Irreführung durch Angaben über den Inbalt eines Verbaltenskodexes}

Als Angaben über den Inhalt eines Verhaltenskodexes sind alle Angaben einzuordnen, welche auf die mit dem Verhaltenskodex eingegangenen Verpflichtungen abstellen. Dabei ist es unerheblich, ob es sich um eine tatsächliche Verpflichtung handelt oder um eine Absichtserklärung. So beispielsweise, wenn ein Unternehmer angibt, sich im Rahmen eines Verhaltenskodexes auf die Einhaltung der ILOKernarbeitsnormen verpflichtet zu haben, tatsächlich aber eine der Regelungen nicht oder nicht vollständig in seinen Verhaltenskodex übernommen hat. Gleiches gilt in dem Fall der Erweiterung der ILO-Kernarbeitsnormen, wenn der Verhaltenskodex nicht ebenfalls entsprechend erweitert wird. Ebenfalls kann es zur Täuschung des Geschäftsverkehrs geeignet sein, wenn es sich bei dem Verhaltenskodex um eine Absichtserklärung handelt, der Unterzeichner jedoch den Eindruck erweckt, sich unmittelbar zur Einhaltung der von der Absichtserklärung umfassten Verhaltensweisen verpflichtet zu haben.

Entsprechende Angaben über den Inhalt eines Verhaltenskodexes sind dabei als Angabe über den Umfang von Verpflichtungen des Unternehmers im Rahmen der Beispiele des $\int 5$ Abs. 1 Satz 2 Nr. 3 UWG einzuordnen. Für dessen Auslegung widerspricht es der Systematik der UGP-Richtlinie und dem Willen der Richtliniengeber, diesen Umstand in der Tradition des deutschen Lauterkeitsrechts vor der Novellierung des UWG im Jahr 2008 ausschließlich auf das Vermögen des Unternehmers zu beziehen und darunter allein Angaben über seine Verbindlichkeiten im finanziellen Sinne wie beispielsweise nicht oder nicht vollständig getilgte Darlehen zu verstehen. ${ }^{803}$ Denn in der UGP-Richtlinie wird dieser Umstand nicht im Rahmen der unternehmensbezogenen Angaben in Art. 6 Abs. 2 lit. f) gemeinsam mit dem Vermögen genannt, sondern in Art. 6 Abs. 2 lit. c) im Zusammenhang mit den Beweggründen des Unternehmers für die geschäftliche Handlung, dem Vertriebsverfahren ${ }^{804}$ sowie den Aussagen und Symbolen im Zusammenhang mit Sponsoring. 805 Zwar geht es aufgrund der Nähe dieses Umstands zum Unternehmer zu weit, die Einordnung in $\int 5$ Abs. 1 Satz 2 Nr. 3 UWG als falsch zu bezeichnen ${ }^{806}$, jedoch muss die Auslegung dort richtlinienkonform erfolgen. Der

\footnotetext{
801 Vgl. Kapitel 6B.I.1

802 Vgl. Kapitel 6B.III.

803 A. A. Bornkamm in: Köhler/Bornkamm, UWG, \ 5, Rn. 5.114a sowie Pfeifer in: Fezer, UWG, $\int 5, \mathrm{Rn} .384$.

804 Vom deutschen Gesetzgeber beide ebenfalls in $\int 5$ Abs. 1 Satz 2 Nr. 3 UWG übernommen.

805 Vom deutschen Gesetzgeber in $\int 5$ Abs. 1 Satz 2 Nr. 4 UWG übernommen.

806 So aber Nordemann, Rn. 300, der diesen Umstand inhaltlich $\int 5$ Abs. 1 Satz 2 Nr. 2 UWG zuordnet, da er nach seiner Ansicht die Bedingungen der Leistung betrifft.
} 
UGP-Richtlinie ist jedoch kein Hinweis zu entnehmen, dass der dortige Wortlaut („Umfang der Verpflichtungen des Gewerbetreibenden") ${ }^{807}$ in irgendeiner Art und Weise einschränkend auszulegen ist. Insbesondere rechtfertigt eine Einordnung des Art. 6 Abs. 2 lit. c) UGP-Richtlinie als Regelung zur Irreführung im Bezug auf vertraglich geschuldete Leistungen keine Einschränkung auf Verpflichtungen des Gewerbetreibenden im Hinblick auf den Umfang der Bindungen zum (potenziellen) Vertragspartner und somit die vertraglichen Verpflichtungen. ${ }^{808}$

\section{2. $\quad$ Irrefübrung durch Angaben zur Überprüfung der Einhaltung eines Verhaltenskodexes}

Neben Angaben zum Inhalt eines Verhaltenskodexes sind zudem solche Angaben praxisrelevant, welche die Überprüfung der Einhaltung eines Verhaltenskodexes betreffen. Denn wie die Billigung eines Verhaltenskodexes steigert es das Vertrauen des Verkehrs, wenn festinstallierte Kontrollmechanismen bezüglich der Einhaltung des Verhaltenskodexes bestehen. ${ }^{809}$ Dies gilt in besonderem Maße für den Fall, dass die Überwachung der Einhaltung des Verhaltenskodexes durch unabhängige Dritte erfolgt. Bestehen die angegebenen Kontrollmechanismen tatsächlich nicht, sind entsprechende Angaben unwahr und damit irreführend. Insbesondere bei Angaben über die Überwachung der Einhaltung durch Dritte ist zudem eine Täuschungseignung auch dann möglich, wenn die Angabe objektiv richtig ist. So beispielsweise dann, wenn der Dritte nicht neutral ist ${ }^{810}$ oder der Geschäftsverkehr aufgrund der Bezeichnung des Dritten einen falschen Eindruck von dessen Bedeutung oder Qualifizierung bekommt. 811 Zudem erwartet der Geschäftsverkehr, dass die mit der Überwachung der Einhaltung des Verhaltenskodexes Beauftragten durch den aufgrund des Verhaltenskodexes Verpflichteten bei der Ausführung ihrer Tätigkeit unterstützt oder zumindest nicht eingeschränkt werden. Insofern ist eine solche Angabe trotz objektiver Richtigkeit täuschungsgeeignet, wenn der durch den Verhaltenskodex verpflichtete dem überwachenden Dritten Dokumente oder Informationen vorenthält. Gleiches gilt, wenn dem überwachenden Dritten der Zutritt beispielsweise zu Produktionsstätten verweigert wird beziehungsweise der durch den Verhaltenskodex Verpflichtete ihn diesbezüglich nicht unterstützt, soweit die Produktion durch Subunternehmer erfolgt. Systematisch sind solche Angabe über die Überprüfung der Einhaltung eines Verhaltenskodexes ebenfalls als Angaben über den Umfang der Verpflichtungen des Unternehmers gemäß \5 Abs. 1 Satz 2 Nr. 3 UWG anzusehen. Denn unter den Begriff des

\footnotetext{
${ }^{807}$ In der englischen Fassung heißt es „extent oft he trader's commitment“, in der französischen „l'etendue des engagements du professionel“.

808 Dreyer in: Harte-Bavendamm/Henning-Bodewig, UWG, \5, E, Rn. 48.

${ }^{809}$ Sogenanntes Monitoring.

810 Vgl. Kapitel 6B.II.1.b.

811 Vgl. Kapitel 6B.II.1.c.
} 
Umfangs der Verpflichtungen eines Unternehmers sind nicht nur Verpflichtungen zu einer bestimmten Handlung zu fassen, sondern auch solche zur Duldung einer Maßnahme.

IV. Irreführung aufgrund einer Verwechslungsgefahr

Eine geschäftliche Handlung ist nach \5 Abs. 2 UWG zudem irreführend, „wenn sie im Zusammenhang mit der Vermarktung von Waren oder Dienstleistungen einschließlich vergleichender Werbung eine Verwechslungsgefahr mit einer anderen Ware oder Dienstleistung oder mit der Marke oder einem anderen Kennzeichen eines Mitbewerbers hervorruft." \5 Abs. 2 UWG wurde ebenfalls im Rahmen der Umsetzung der UGP-Richtlinie in das UWG eingefügt und hat die bis dahin geltende lauterkeitsrechtliche Gesetzeslage zur Irreführung aufgrund einer Verwechslungsgefahr erheblich verändert. ${ }^{812}$ Im Hinblick auf die Regelungen zur Irreführung durch aktives Handeln aus $\int 5$ UWG stellt sie eine Besonderheit dar, da sie ohne das Tatbestandsmerkmal der Täuschungseignung auskommt. ${ }^{813}$ Grund hierfür ist, dass die Täuschungseignung den von ihr geregelten Sachverhalten aufgrund des notwendigen Tatbestandsmerkmales der Verwechslungsgefahr ${ }^{814}$ immanent ist. Die Relevanz der Norm für geschäftliche Handlungen im Zusammenhang mit Verhaltenskodizes ergibt sich insbesondere daraus, dass die Logos von Verhaltenskodizes oftmals als Marken eingetragen sind ${ }^{815}$ und damit eine entsprechende Verwechslungsgefahr besteht. Die Unlauterkeit einer nach \5 Abs. 2 UWG irreführenden geschäftlichen Handlung ergibt sich aus $\int 5$ Abs. 1 Satz 1 UWG, ihre Unzulässigkeit aus $\int 3$ Abs. 1 UWG. ${ }^{816}$

\section{Beschränkung der betroffenen geschäftlichen Handlungen}

\ 5 Abs. 2 UWG erfasst entsprechend der Vorgabe aus Art. 6 Abs. 2 lit. a) UGPRichtlinie nicht alle geschäftlichen Handlungen i. S. d. \ 2 Abs. 1 Nr. 1 UWG ${ }^{817}$, sondern nur solche, die „im Zusammenhang mit der Vermarktung von Waren oder Dienstleistungen" stehen. ${ }^{818}$ Damit ist begrifflich im Regelfall zunächst einmal der Zeitbereich nach Abschluss eines Geschäfts ausgeschlossen, da die Vermarktung diesem

\footnotetext{
812 Bornkamm in: Köhler/Bornkamm, UWG, \ 5, Rn. 1.85.

813 Vgl. zum Unterschied zu den nach $\int 5$ Abs. 1 Satz 2 UWG irreführenden Handlungen Kapitel 5B.II.

814 Vgl. nachfolgend Kapitel 6B.IV.2.

815 So ist das Logo des Responsible Care Programms (vgl. Kapitel 1C.II.2) unter der Markennummer 47803 in das Register des DPMA eingetragen.

816 Vgl. zu den Voraussetzungen der Unzulässigkeit Kapitel 5B.I.

817 Vgl. Kapitel 4A.

818 Eine solche Beschränkung einer Norm auf bestimmte geschäftliche Handlungen findet sich auch in $\int 5$ a Abs. 3 UWG, vgl. nachfolgend Kapitel 6C.II.1.a.aa.
} 
immer vorgelagert ist. ${ }^{819}$ Gegeben ist ein solcher Zusammenhang bei jeglicher Art der Vermarktung und somit bei allen Formen der Werbung, aber auch bei der Produktbeschreibung und sogar der Produktgestaltung, da Letztere einen erheblichen Anteil an der Vermarktung und Platzierung hat. ${ }^{820}$ Keine Beschränkung besteht dahingegen hinsichtlich des persönlichen Anwendungsbereiches ${ }^{821}$, so dass sowohl geschäftliche Handlungen im b2b- als auch im b2c-Bereich erfasst werden. ${ }^{822}$ Nicht erfasst werden dahingegen rein redaktionelle Berichte, die verschiedene Waren oder Dienstleistungen vergleichen. ${ }^{823}$ Dieser Ausschluss beruht aber nicht auf dem eingeschränkten sachlichen Anwendungsbereich des \5 Abs. 2 UWG, sondern auf der Unanwendbarkeit des UWG auf redaktionelle ÄuBerungen von Unternehmen oder anderen Personen. ${ }^{824}$

\section{Verwechslungsgefahr}

Ausreichend für die Annahme der Irreführung nach $\int 5$ Abs. 2 UWG ist das Bestehen einer Verwechslungsgefahr und somit der Gefahr, dass der von einer geschäftlichen Handlung betroffene Adressatenkreis die mit der geschäftlichen Handlung vermarktete Ware oder Dienstleistung mit Waren oder Dienstleistungen, Marken oder anderen Kennzeichen eines anderen Unternehmens verwechselt. Es ist somit nicht notwendig, dass es tatsächlich zu einer Verwechslung kommt. ${ }^{825}$ Evident gegeben ist eine solche Verwechslungsgefahr, wenn die Waren oder Dienstleistungen identisch sind. ${ }^{826}$ Ausreichend ist aber ebenfalls, dass die betroffenen Verkehrskreise aufgrund der Verwendung vom Marken und anderen Kennzeichen den Eindruck gewinnen können, dass die Waren oder Dienstleistungen aus demselben Unternehmen stammen, wobei hierfür keine rechtliche Einheit vorausgesetzt wird, sondern organisatorisch oder wirtschaftlich enge Beziehungen

${ }^{819}$ Dreyer in: Harte-Bavendamm/Henning-Bodewig, UWG, \5, J, Rn. 19, die zu Recht darauf hinweist, dass dies nicht gilt, wenn in diesem Zeitbereich die Vermarktung anderer Waren oder Dienstleistungen angestrebt wird.

${ }^{820}$ Dreyer in: Harte-Bavendamm/Henning-Bodewig, UWG, \5, J, Rn. 19.

821 Vgl. Kapitel 4C.

822 Dreyer in: Harte-Bavendamm/Henning-Bodewig, UWG, \5, J, Rn. 19.

${ }^{823}$ Sosnitza in: Piper/Ohly/Sosnitza, UWG, \5, Rn. 704.

${ }^{824}$ Regierungsentwurf und Begründung zum UWG vom 20.08.2008, BT.-Drs. 16/10145, S. 21.

825 Dreyer in: Harte-Bavendamm/Henning-Bodewig, UWG, \5, J, Rn. 20, Sosnitza in: Piper/Ohly/Sosnitza, UWG, \5, Rn. 703, a. A. Bornkamm in: Köhler/Bornkamm, UWG, \5, Rn. 4.238, der im Hinblick auf den Wortlaut der UGP-Richtlinie in der englischen (,which creates confusion") und der französischen (, ,créant une confusion") abstellt.

826 Sosnitza in: Piper/Ohly/Sosnitza, UWG, \5, Rn. 703. 
ausreichen. ${ }^{827}$ Es besteht somit eine erhebliche Schnittmenge mit irreführenden Angaben über die betriebliche Herkunft gemäß \ 5 Abs. 1 Satz 2 Nr. 1 UWG. ${ }^{828}$

Von besonderer Relevanz für geschäftliche Handlungen im Zusammenhang mit Verhaltenskodizes ist die Einschränkung, dass nicht jede Verwechslungsgefahr irreführend ist, sondern nur die mit Waren und Dienstleistungen sowie Marken und anderen Kennzeichen von Mitbewerbern und damit von Unternehmern. Während Waren und Dienstleistungen in aller Regel Unternehmern zuzuordnen sind, gilt dies nicht in gleichem Maße für Marken und andere Kennzeichen. Denn diese können auch (juristischen) Personen zuzuordnen sein, die nicht unter den Unternehmerbegriff des UWG fallen. ${ }^{829}$ Nicht von $\int 5$ Abs. 2 UWG umfasst ist somit die Verwendung eines Logos, welches aufgrund einer Wort-/Bildmarke oder einer Bildmarke nach \3 Abs. 1 MarkenG für einen Verband oder eine NRO geschützt ist. Gerade im Hinblick auf Verhaltenskodizes werden die Logos, welche die durch den Verhaltenskodex verpflichteten Unternehmen führen dürfen, aber oftmals durch Verbände oder NROen vergeben und von diesen auch markenrechtlich geschützt. ${ }^{830}$ Selbst wenn man diese noch unter ein weites Verständnis des Unternehmerbegriffs fassen könnte, wären sie jedoch in jedem Fall keine Mitbewerber. Denn als solche erkennt das UWG nur einen Unternehmer an, „der mit einem oder mehreren Unternehmern als Anbieter oder Nachfrager von Waren oder Dienstleistungen in einem konketen Wettbewerbsverhältnis steht.“831

\section{Zusammenfassung}

\5 UWG als allgemeinem Irreführungsparagraphen kommt auch bei der Bewertung von geschäftlichen Handlungen im Zusammenhang mit Verhaltenskodizes eine besondere Bedeutung zu. So sind die Angaben im Zusammenhang mit Verhaltenskodizes aus Nr. 1 und Nr. 3 des Anhangs zu $\int 3$ Abs. 3 UWG, wenn sie im b2b-Verhältnis getätigt werden und objektiv unwahr sind, nach $\int 5$ Abs. 1 Satz 2 Nr. 3 UWG irreführend und damit unlauter. Hierdurch wird ein zu großes Ungleichgewicht in der Bewertung identischer geschäftlicher Handlungen zwischen den Bereichen b2b und b2c verhindert. Darüber hinaus ermöglicht $\int 5$ Abs. 1 Satz 2 Nr. 3 UWG die Einordnung von geschäftlichen Handlungen sowohl im

827 Sosnitza in: Piper/Ohly/Sosnitza, UWG, \ 5, Rn. 703.

828 Dreyer in: Harte-Bavendamm/Henning-Bodewig, UWG, $\int 5$, J, Rn. 18, weitergehend Sosnitza in: Piper/Ohly/Sosnitza, UWG, $\int 5$, Rn. 703, dahingehend, dass der Begriff der Verwechslungsgefahr aus $₫ 5$ Abs. 2 nichts anderes meint als eine Irreführung über die betriebliche Herkunft.

829 Zum Begriff des Unternehmers vgl. die Legaldefinition in $\$ 2$ Abs. 1 Nr. 6 UWG sowie Kapitel 3B.

830 So ist beispielsweise das Logo des Responsible Care Programms (vgl. Kapitel 1C.II.2) unter der Markennummer 47803 für das European Chemical Industry Council in das Register des DPMA eingetragen.

831 \ 2 Abs. 1 Nr. 3 UWG. Die UGP-Richtlinie enthält keine Definition des Begriffs des Mitbewerbers. 
b2b-, als auch im b2c-Bereich, welche die Angaben im Zusammenhang mit Verhaltenskodizes aus Nr. 1 und Nr. 3 des Anhangs zu $₫ 3$ Abs. 3 UWG enthalten, als irreführend, wenn diese Angaben zwar objektiv richtig, jedoch aufgrund besonderer Umstände trotzdem zur Täuschung geeignet sind. Auch hierdurch wird ein Wertungswiderspruch innerhalb des UWG verhindert. Denn eine geschäftliche Handlung, die stets unzulässig ist, weil sie eine bestimmte unwahre Angabe enthält, muss zumindest auch unlauter sein, wenn die entsprechende Angabe zwar objektiv richtig ist, aber trotzdem irreführend.

Darüber hinaus ist $\int 5 \mathrm{UWG}$ von überragender Bedeutung für sämtliche im Zusammenhang mit Verhaltenskodizes getätigte unwahre oder sonstige zur Täuschung geeignete Angaben in geschäftlichen Handlungen, die über die Angaben aus Nr. 1 und Nr. 3 des Anhangs zu $₫ 3$ Abs. 3 UWG hinausgehen. So erfasst $\ 5$ Abs. 1 Satz 2 Nr. 3 UWG Angaben über den Inhalt von Verhaltenskodizes und damit die für das Unternehmen bestehenden Verpflichtungen aus diesen sowie Angaben zur Überprüfung der Einhaltung der Verpflichtungen aus Verhaltenskodizes. Entsprechende Angaben sind irreführend und damit unlauter, wenn sie unwahr oder aus sonstigen Gründen zur Täuschung geeignet sind.

Eingeschränkt ist die Bedeutung von $\ 5$ UWG dahingegen für die Beurteilung der Irreführung von geschäftlichen Handlungen im Zusammenhang mit Verhaltenskodizes im Hinblick auf die durch diese hervorgerufene Verwechslungsgefahr mit anderen Verhaltenskodizes. Grund hierfür ist, dass $\ 5$ Abs. 2 UWG nur die Verwechslungsgefahr mit Waren oder Dienstleistungen sowie Marken und anderen Kennzeichen von Mitbewerbern erfasst, die im Zusammenhang mit Verhaltenskodizes geschützten Marken und verwendeten Kennzeichen jedoch oftmals Verbänden, NROen oder anderen Institutionen zuzuordnen sind, die nicht als Mitbewerber i. S. d. $\$ 2$ Abs. 1 Nr. 3 UWG eingeordnet werden können. In dem verbleiben Anwendungsbereich trägt $₫ 5$ Abs. 2 UWG jedoch zum Schutz des Wettbewerbes bei, indem er die bei Trittbrettfahrern verbreitete Vorgehensweise, sich bei der Gestaltung ihrer Zeichen an bestehenden und renommierten Marken oder anderen Kennzeichen zu orientieren, um von diesen zu profitieren, als irreführend einordnet.

\section{Irreführung durch Unterlassen bei geschäftlichen Handlungen im Zusammenhang mit Verhaltenskodizes, $\mathbb{5}$ a UWG}

Das UWG schützt die Entscheidungsfreiheit nicht nur vor Irreführungen durch aktives Handeln, sondern auch vor solchen durch das Verschweigen einer Tatsache beziehungsweise das Vorenthalten einer wesentlichen Information und damit durch Unterlassen. Hatte das UWG 2004 die Irreführung durch Unterlassen noch eher beiläufig mit einem Satz im Rahmen des allgemeinen Irreführungsparagra- 
phen $^{832}$ geregelt ${ }^{833}$, wurde für die Thematik im Rahmen der Änderung des UWG zur Umsetzung der UGP-Richtlinie im Jahr 2008 mit \5a UWG ein eigener Paragraph eingeführt, um die ausführliche Regelung aus Art. 7 UGP-Richtlinie umzusetzen. ${ }^{834}$ Abweichend vom Grundsatz der einheitlichen Geltung des UWG sollten die Vorgaben der UGP-Richtlinie jedoch nur für geschäftliche Handlungen gegenüber Verbrauchern Geltung erhalten, ${ }^{835}$ um den kaufmännischen Verkehr nicht mit in erster Linie dem Verbraucherschutz dienenden Informationsanforderungen zu belasten. ${ }^{836} \mathrm{Um}$ klarzustellen, dass für geschäftliche Handlungen gegenüber Unternehmern die alte Rechtslage im Wesentlichen weiterhin gelten soll, wurde der Regelung als $\int 5$ a Abs. 1 UWG die nur um das Notwendigste ${ }^{837}$ angepasste Regelung aus \5 Abs. 2 Satz 2 UWG 2004 vorangestellt. ${ }^{838}$ Die Vorgaben der UGP-Richtlinie für geschäftliche Handlungen gegenüber Verbrauchern wurden dahingegen durch die Absätze 2 bis 4 umgesetzt. ${ }^{839}$ Dabei hat es der Gesetzgeber jedoch versäumt, diese Unterscheidung im Gesetzestext ausdrücklich hervorzuheben, so dass Köhler zuzustimmen ist, wenn er das Verhältnis von \5a Abs. 1 und Abs. 2 UWG als „nicht sehr glücklich“ bezeichnet. ${ }^{840}$ Denn die Regelung ist nicht aus sich selbst heraus verständlich, sondern nur unter Berücksichtigung der Normentwicklung.

Unabhängig von der Unterscheidung zwischen geschäftlichen Handlungen im b2b- sowie im b2c-Bereich, welche auch der Untersuchung der Irreführung durch Unterlassen in dieser Arbeit zugrunde liegt, ist für die Anwendbarkeit des $\int 5 a$ UWG entscheidend, dass die Beeinflussung der Entscheidungsfreiheit tatsächlich auf einem Unterlassen beruht. Insofern ist oftmals eine Abgrenzung dahingehend vorzunehmen, ob die Beeinflussung durch Verschweigen einer Tatsache beziehungsweise Vorenthaltung einer Information erfolgt oder nicht doch

832 \ 5 Abs. 2 Satz 2 UWG 2004.

833 Köhler, NJW 2008, 3032, 3034, Regierungsentwurf und Begründung zum UWG vom 20.08.2008, BT.-Drs. 16/10145, S. 17.

834 Regierungsentwurf und Begründung zum UWG vom 20.08.2008, BT.-Drs. 16/10145, S. 17.

835 Puchinger, S. 78.

836 Regierungsentwurf und Begründung zum UWG vom 20.08.2008, BT.-Drs. 16/10145, S. 25.

837 Ausgetauscht wurde lediglich das vormals geltende Merkmal „Bedentung für die Entscheidung zum Vertragsschluss" durch das neue Merkmal „Bedentung für die geschäftliche Entscheidung“. Dies war aufgrund des erweiterten zeitlichen Anwendungsbereiches des UWG auf nachvertragliche geschäftliche Handlungen notwendig, Regierungsentwurf und Begründung zum UWG vom 20.08.2008, BT.-Drs. 16/10145, S. 25.

838 Zwar umfasst \5a Abs. 1 UWG auch das Verschweigen von Tatsachen gegenüber Verbrauchern, jedoch sind geschäftliche Handlungen gegenüber Verbrauchern immer richtlinienkonform auszulegen. Entsprechend beschränkt sich die Anwendbarkeit des $\int 5 \mathrm{a}$ Abs. 1 UWG faktisch auf Unternehmer und sonstige Marktteilnehmer. Dies gilt aufgrund der Vollharmonisierung durch die UGP-Richtlinie sowohl für den Fall, dass $\int 5$ a Abs. 1 UWG über den Regelungsumfang der UGP-Richtlinie hinausgeht, als auch wenn er hinter diesem zurückbleibt, vgl. Kapitel 2B.I.1.

839 Regierungsentwurf und Begründung zum UWG vom 20.08.2008, BT.-Drs. 16/10145, S. 25.

840 Bornkamm in: Köhler/Bornkamm, UWG, \ 5a, Rn. 6. Entsprechend auch Beater, Rn. 1443. 
aufgrund einer missverständlichen positiven Information. ${ }^{841}$ Denn in letzterem Fall muss die missverständliche Angabe nach den Vorgaben des $\int 5 \mathrm{UWG}^{842}$ geprüft werden. ${ }^{843} \mathrm{Da}$ eine mögliche Irreführung aufgrund des Verschweigens einer Tatsache beziehungsweise dem Vorenthalten einer wesentlichen Information nur anhand einer bestimmten Tatsache beziehungsweise Information beurteilt werden kann, beschränkt sich diese Arbeit in diesem Bereich auf die Tatsache beziehungsweise Information, ob eine Bindung an einen Verhaltenskodex besteht oder nicht besteht sowie auf die Information, ob gegen einen Verhaltenskodex verstoBen wurde.

\section{Geschäftliche Handlungen gegenüber Unternehmern}

Bei der Betrachtung des für die Irreführung durch Unterlassen gegenüber Unternehmern maßgeblichen $\int 5 \mathrm{a}$ Abs. $1 \mathrm{UWG}$ fällt zunächst auf, dass dieser selbst keinen Unlauterkeitstatbestand enthält ${ }^{844}$, sondern dem Wortlaut nach nur eine Bewertungsmaßgabe für die Frage, ob das Verschweigen einer Tatsache irreführend ist. Dies beruht auf der Tatsache, dass die Regelung — lediglich im Hinblick auf den zeitlichen Anwendungsbereich angepasst ${ }^{845}$ — wörtlich aus $\int 5$ Abs. 2 Satz 2 UWG 2004 übernommen wurde und der Gesetzgeber darauf verzichtet hat, eine Formulierung voranzustellen, welche dem bis dahin für $\int 5$ Abs. 2 Satz 2 UWG 2004 geltende Grundtatbestand aus \5 Abs. 1 UWG 2004 entsprach. ${ }^{846}$ Infolgedessen muss zur Herstellung einer durchgängigen Rechtsfolgenkette $^{847}$ nunmehr auf $\int 5$ Abs. 1 Satz 1 UWG zurückgegriffen werden. ${ }^{848}$ Dass $\$ 5a Abs. 1 UWG seinem Wortlaut nach auch keine tatbestandliche Festlegung einer irreführenden Handlung enthält ${ }^{849}$ ist dahingegen unerheblich, da die Formulierung entsprechend ausgelegt werden kann.

Hinsichtlich der Frage, wann das Verschweigen einer Tatsache irreführend ist, kann aufgrund der bewussten Übernahme des $\int 5$ Abs. 2 Satz 2 UWG 2004 die zu diesem ergangene Rechtsprechung ebenso zur Auslegung von \5a Abs. 1 UWG

\footnotetext{
841 Nordemann, Rn. 376.

$842 \mathrm{Vgl}$. Kapitel 6B.

843 A. A: Bornkamm in: Köhler/Bornkamm, UWG, \5a, Rn. 6, der die von \5a Abs. 2 UWG erfassten Tatbestände nicht als Unterlassungsdelikte ansieht, da die Irreführung bei Ihnen auch unmittelbar durch ein positives Tun begründet würde.

${ }^{844}$ Bornkamm in: Köhler/Bornkamm, UWG, \5a, Rn. 6a. Es fehlt eine Formulierung wie „,unlauter bandelt, wer...".

845 Vgl. vorstehende Einleitung unter Kapitel 6C, insbesondere auch Fn. 837.

846 Dieser bestimmte wie sinngemäß der heutige $\int 5$ Abs. 1 Satz 1 UWG das , unlauter $\{\ldots\}$ handelt, wer irreführend wirbt."

${ }^{847}$ Irreführend $\rightarrow$ Unlauter $\rightarrow$ Unzulässig.

848 Bornkamm in: Köhler/Bornkamm, UWG, \5a, Rn. 6a.

${ }^{849}$ So beispielsweise durch eine Formulierung wie in $\int 5$ Abs. 1 Satz 2 UWG „Eine geschäftliche Handlung ist irrefübrend, wenn $\{\ldots\}$. .
} 
herangezogen werden ${ }^{850}$ wie die Rechtsprechung zur Irreführung durch Unterlassen nach dem UWG 1909, da diese mit \5 Abs. 2 Satz 2 UWG 2004 als Rechtssatz in das UWG übernommen werden sollte. ${ }^{851}$ Dementsprechend stellt das Verschweigen einer Tatsache i. S. d. S $5 \mathrm{a}$ Abs. 1 UWG nur dann ein irreführendes Unterlassen dar, wenn für den Verantwortlichen der geschäftlichen Handlung eine Aufklärungspflicht besteht. ${ }^{852}$ Eine solche Aufklärungspflicht kann sich aus Gesetz, Vertrag, vorangegangenem Tun oder aber aus der besonderen Bedeutung ergeben, die der verschwiegenen Tatsache nach Auffassung des Verkehrs für die geschäftliche Entscheidung zukommt. ${ }^{853}$ Ein generelles Informationsgebot sollte durch die Norm dahingegen ausdrücklich nicht geschaffen werden. ${ }^{854}$ Denn Informationspflichten sollten nach dem UWG 2004 nur bei Verkaufsförderungsmaßnahmen einschließlich Preisausschreiben und Gewinnspielen bestehen. ${ }^{85}$ Dies erachtete der historische Gesetzgeber als ausreichend, da er davon ausging, dass der Geschäftsverkehr ,nicht ohne weiteres die Offenlegung aller, insbesondere der weniger vorteilhaften Eigenschaften einer W are oder Leistung erwartet." ${ }^{\text {"856 }}$

\section{Aufklärungspflicht über die bestehende oder nicht (mehr) bestehende Bindung an einen Verhaltenskodex}

Hinsichtlich der Tatsache, dass ein Unternehmen an einen Verhaltenskodex gebunden ist oder nicht, besteht keine gesetzliche Aufklärungspflicht. ${ }^{857}$ Ebenfalls kommt dieser Tatsache keine solche besondere Bedeutung für geschäftliche Entscheidung eines Unternehmers zu, so dass keine allgemeingültige Verpflichtung diesbezüglich besteht. Denn auch wenn die Bindung an einen Verhaltenskodex (teilweise) im Rahmen von geschäftlichen Entscheidungen von Unternehmern Beachtung findet, kann die notwendige besondere Bedeutung für die geschäftliche Entscheidung nur wirtschaftlichen Merkmalen wie dem Preis und der Qualität zugestanden werden. So hat der BGH selbst für die stärker auf die Entscheidungsfreiheit einwirkenden Sachverhalte aus der Fallgruppe der Kopplungsangebote ${ }^{858}$

\footnotetext{
850 Beater, Rn. 1443, Bornkamm in: Köhler/Bornkamm, UWG, \5a, Rn. 8.

851 Regierungsentwurf und Begründung zum UWG vom 22.08.2004, BT.-Drs. 15/1487, S. 20.

852 Vgl. Fn. 851, so bspw. festgestellt durch Urteil des BGH v. 03.12.1998 - I ZR 63/96 (OLG Hamburg) (Auslaufmodelle I) in GRUR 1999, $757 \mathrm{ff}$.

853 Regierungsentwurf und Begründung zum UWG vom 22.08.2004, BT.-Drs. 15/1487, S. 20, welche statt auf die geschäftliche Entscheidung auf den Kaufentschluss abstellt.

854 Regierungsentwurf und Begründung zum UWG vom 22.08.2004, BT.-Drs. 15/1487, S. 20.

855 Geregelt in \ 4 Nr. 4 und Nr. 5 UWG 2004, vgl. Regierungsentwurf und Begründung zum UWG vom 22.08.2004, BT.-Drs. 15/1487, S. 20.

856 Regierungsentwurf und Begründung zum UWG vom 22.08.2004, BT.-Drs. 15/1487, S. 19.

857 Andernfalls wären entsprechende Verhaltensweisen auch als Rechtsbruch nach $\$ 4$ Nr. 11 UWG zu behandeln, vgl. Kapitel 6A.

${ }^{858}$ Kopplungsangebote sind solche Angebote, bei welchen verschiedene Waren und Dienstleistungen in ein Gesamtangebot zusammengefasst werden. Oftmals durch Zugabe einer kostenfreien
} 
entschieden, dass eine Verpflichtung zu aufklärenden Maßnahmen über das an die geschäftliche Entscheidung gekoppelte soziale, sportliche, kulturelle oder ökologische Engagement nur dann besteht, „wenn andernfalls die Gefabr einer unlauteren Beeinflussung des Verbrauchers durch Täuschung über den tatsäcblichen Wert des Angebots, insbesondere über den Wert der angebotenen Zusatzleistung, gegeben ist. "859 Wenn jedoch bereits bei geschäftlichen Handlungen gegenüber Verbrauchern nur eine Täuschung über den finanziellen Wert des Angebots anerkannt wird und damit wirtschaftliche Erwägungen herausgestellt werden, so muss dies auch beziehungsweise erst Recht für geschäftliche Handlungen gegenüber Unternehmern gelten.

Eine Aufklärungspflicht über die Bindung an einen Verhaltenskodex kann somit nur in Einzelfällen aufgrund einer entsprechenden vertraglichen Verpflichtung oder aufgrund von Ingerenz bestehen. Im Hinblick auf eine entsprechende vertragliche Verpflichtung kommt es dabei auf die konkrete Ausgestaltung der Verpflichtung an. Ausreichend wäre beispielsweise die Verpflichtung in einem als Vertrag einzuordnenden Verhaltenskodex ${ }^{860}$, im Rahmen von (bestimmten) geschäftlichen Handlungen auf die Bindung an den Verhaltenskodex hinzuweisen beziehungsweise nach Ende der Bindung an den Verhaltenskodex darauf hinzuweisen, dass diese Bindung nicht mehr besteht. Selbstverständlich muss sich eine solche Verpflichtung aber nicht direkt aus dem Verhaltenskodex ergeben. Vielmehr ist jede entsprechende Verpflichtung in einem Vertrag ausreichend. Denkbar erscheint eine solche Verpflichtung beispielsweise in Franchiseverträgen, Vertriebsvereinbarungen und anderen Konstellationen, in denen das öffentliche Tätigwerden des einen Vertragspartners unmittelbare Auswirkungen auf den anderen hat oder sogar als dessen Tätigwerden verstanden wird.

Eine Aufklärungspflicht aus Ingerenz setzt voraus, dass im Rahmen vorheriger geschäftlicher Handlungen eine besondere Erwartungshaltung geschaffen wurde. ${ }^{861}$ So beispielsweise durch die Herausstellung bestimmter Umstände oder Merkmale, welche sich jedoch in der Folgezeit verändert haben. ${ }^{862}$ Hinsichtlich der Frage, ob eine Erwartungshaltung des Verkehrs entstanden ist, muss berücksichtigt werden, dass Verhaltenskodizes Teil des grundsätzlichen Selbstverständnisses eines Unternehmens sind und aus diesem Grund gerade als unveränderliche Maßgabe für sämtliche, auch zukünftige Verhaltensweisen des Unternehmens wahrgenommen werden. Nicht ohne Grund wird im Zusammenhang mit Verhal-

oder besonders kostengünstigen Ware oder Dienstleistung zum Hauptprodukt des Anbietenden.

${ }^{859}$ Urteil des BGH v. 26.10.2006 - I ZR 33/04 (OLG Hamm) (Regenwaldprojekt I) in GRUR 2007, S. 247,250, Tz. $24 \mathrm{f}$.

${ }^{860} \mathrm{Vgl}$. Kapitel 1D.

${ }^{861}$ Dreyer in: Harte-Bavendamm/Henning-Bodewig, UWG, \5, C, Rn. 798, 1. Nachdruck 2004.

862 Dreyer in: Harte-Bavendamm/Henning-Bodewig, UWG, S 5, C, Rn. 798, 1. Nachdruck 2004 unter Verweis auf Urteil des BGH v. 31.05.1957 - I ZR 163/55 (OLG Düsseldorf) (Außenleuchte) $=$ GRUR 1958, 30, 31 . 
tenskodizes oftmals auch von der DNA eines Unternehmens gesprochen. ${ }^{863}$ Entsprechend dürfte bereits ein einmaliger Hinweis auf die Bindung an einen Verhaltenskodex beim Verkehr die Erwartungshaltung begründen, dass die Bindung auch in Zukunft besteht. Das Bestehen einer solchen Erwartungshaltung des Verkehrs bedeutet jedoch nicht, dass von nun an bei jeder geschäftlichen Handlung auf die Bindung an den Verhaltenskodex hingewiesen werden muss. Denn die Erwartung des Verkehrs betrifft nur die Bindung an den Verhaltenskodex, nicht aber den dauerhaften Hinweis auf diese. Eine Aufklärungspflicht aus vorangegangenem Tun kann vielmehr nur dann bestehen, wenn sich eine Änderung bezüglich der besonders herausgestellten Umstände oder Merkmale ergibt. Dies ist im Bezug auf die Bindung an einen Verhaltenskodexes nur insoweit relevant, als die Bindung ausdrücklich herausgestellt wurde und später nicht mehr besteht. Denn die andere Alternative würde voraussetzen, dass ein Unternehmen die fehlende Bindung an einen Verhaltenskodex in besonderer Weise herausstellt und sich eine Erwartungshaltung des Verkehrs entwickelt hat, nach welcher das Unternehmen nicht an einen Verhaltenskodex gebunden ist.

Selbstverständlich darf sich aus einem einmaligen Hinweis auf die Bindung an einen Verhaltenskodex nach Beendigung dieser Bindung jedoch keine dauerhafte Verpflichtung eines Unternehmens ergeben, im Rahmen von sämtlichen nachfolgenden geschäftlichen Handlungen darüber aufzuklären, nunmehr nicht mehr an den Verhaltenskodex gebunden zu sein. Vielmehr muss sowohl im Hinblick auf den Zeitraum als auch im Hinblick auf die von der Aufklärungspflicht betroffenen geschäftlichen Handlungen eine den Interessen des Geschäftsverkehrs und den Interessen des Unternehmens angemessene Einschränkung getroffen werden. Dabei muss zugunsten des Unternehmens berücksichtigt werden, dass es unverhältnismäßig wäre, dieses zu verpflichten, die negative Merkmale der eigenen Waren oder Dienstleistungen mit der gleichen Intensität zu kommunizieren wie die positiven Merkmale. Entsprechend muss es grundsätzlich ausreichen, dass der Hinweis auf die Beendigung der Bindung an einen Verhaltenskodex (nur) im Rahmen einer Pressemitteilung oder im Internetauftritt des Unternehmens erfolgt, obwohl zuvor beispielsweise auch in Werbemaßnahmen auf den Verhaltenskodex hingewiesen worden ist. Eine Ausnahme hiervon ist nur dann berechtigt, wenn das Unternehmen zuvor in besonderer Intensität auf die Bindung an den Verhaltenskodex hingewiesen hat. So beispielsweise, wenn der Verhaltenskodex in den Mittelpunkt einer Werbemaßnahme oder -kampagne gestellt wurde. Im Hinblick auf den Zeitraum hat die Interessensabwägung dahingegen zugunsten des Ge-

863 So beispielsweise die Studie von Anna Peters für die Bertelsmann Stiftung „Die Wege aus der Krise - CSR als strategisches Rüstzeng für die Zukunft", S. 11, abrufbar unter http://www.bertelsmannstiftung.de/cps/rde/xchg/SID-8493BAF0-D1E80665/bst/hs.xsl/downloads.htm?sort $=$ Titel\&sortorder $=$ asc\&chunk $=2 \&$ suchbegriff $=$ anna $\% 20$ peters $\% 20$ wege $\% 20$ aus $\% 20 \mathrm{der} \% 20 \mathrm{kri}$ se\&suchrubrik, zuletzt abgerufen am 16.04.2013. 
schäftsverkehrs auszufallen, so dass ein einmaliger Hinweis nicht ausreichend ist. Über welchen genauen Zeitraum eine Aufklärungspflicht besteht, muss jedoch im Einzelfall unter Berücksichtigung der Intensität und Dauer des vorherigen Hinweises auf die Bindung an den Verhaltenskodex ermittelt werden. Als Obergrenze sollte hierfür jedoch der Zeitraum der Bindung an den Verhaltenskodex festgesetzt werden.

\section{Aufklärungspflicht über den Verstoß gegen einen Verhaltenskodex}

Ebenso wenig wie für den Hinweis auf die Bindung an einen Verhaltenskodex besteht eine gesetzliche Aufklärungspflicht über die Tatsache, gegen einen Verhaltenskodex verstoßen zu haben. Denn bei dem Verstoß gegen einen solchen handelt es sich maximal um einen Vertragsverstoß ${ }^{864}$, für welchen keine gesetzlichen Offenbarungspflichten bestehen. Gleichfalls hat der Verstoß gegen einen Verhaltenskodex auch keine besondere Bedeutung für die geschäftliche Entscheidung von Unternehmern, da diese im Wesentlichen auf wirtschaftlichen Erwägungen beruht. ${ }^{865}$ Selbstverständlich kann sich eine dahingehende Aufklärungspflicht jedoch aus dem Verhaltenskodex, falls dieser Vertragsqualität hat, oder einem von diesem unabhängigen Vertrag ergeben, wenn jeweils eine entsprechende Verpflichtung vorgesehen ist. 866 Im Hinblick auf eine Aufklärungspflicht aus Ingerenz ist festzustellen, dass für eine solche allein der vorherige Hinweis auf die Bindung an einen Verhaltenskodex nicht ausreichen kann. Denn der Geschäftsverkehr erwartet aufgrund dessen nur, dass die Bindung fortbesteht und nicht, dass er über einen Verstoß gegen den Verhaltenskodex informiert wird. Eine Aufklärungspflicht über Verstöße gegen einen Verhaltenskodex kann daher nur bestehen, wenn durch das vorangegangene Tun ein besonderer Vertrauenstatbestand geschaffen wurde, dass die Verpflichtungen aus einem Verhaltenskodex immer eingehalten werden.

\section{Geschäftliche Handlungen gegenüber Verbrauchern}

Auch wenn $\int 5 a$ Abs. 1 UWG seinem Wortlaut nach auch für irreführende Unterlassungen in geschäftlichen Handlungen gegenüber Verbrauchern Anwendung findet, richtet sich deren Unlauterkeit ausschließlich nach den Absätzen 2 bis 4 des $\int 5 \mathrm{a}$ UWG. Denn die Irreführung durch Unterlassen wird durch den mit diesen in das deutsche Recht umgesetzten Art. 7 UGP-Richtlinie aufgrund der mit der UGP-Richtlinie angestrebten Vollharmonisierung ${ }^{867}$ umfassend geregelt. Abweichungen von dieser vollständigen europäischen Angleichung sind im Hinblick

\footnotetext{
864 Vgl. Kapitel 1D.

865 Vgl. Kapitel 6C.I.1.

866 Vgl. Kapitel 6C.I.1.

867 Vgl. Kapitel 2B.I.1.
} 
auf die Irreführung durch Unterlassen nur insoweit zulässig, als Mitgliedstaaten ,für bestimmte Produkte, zum Beispiel Sammlungsstücke oder elektrische Geräte, die wesentlichen Kennzeichen festlegen (dürfen, Ergänzung d .d. Verf.), deren Weglassen bei einer Aufforderung zum Kauf rechtserbeblich wäre."868 Von dieser Ermächtigung hat der deutsche Gesetzgeber jedoch keinen Gebrauch gemacht. ${ }^{869}$ Daher sind die Absätze 2 bis 4 des $\int 5$ a UWG so auszulegen, dass sie weder hinter den Maßgaben des Art. 7 UGP-Richtlinie zurückbleiben noch über diese hinausgehen. \5a Abs. 1 UWG kann daher lediglich als ergänzende Rechtsgrundlage herangezogen werden, wenn sowohl seine als auch die Voraussetzungen der Abs. 2 bis 4 des $\int 5 \mathrm{a}$ UWG erfüllt sind. Ein eigener Anwendungsbereich für geschäftliche Handlungen gegenüber Verbrauchern verbleibt für ihn jedoch nicht.

Im Rahmen dieses für irreführende Unterlassungen gegenüber Verbrauchern maßgeblichen Regelungskonvolutes stellt \5a Abs. 2 UWG die Grundregelung dar. Dieser enthält sämtliche Tatbestandsvoraussetzungen und setzt als Rechtsfolge ohne den Zwischenschritt der Irreführung die Unlauterkeit der geschäftlichen Handlung fest. Diese Rechtsfolge rechtfertigt die Norm wie \5a Abs. 1 UWG mit dem Schutz der Entscheidungsfreiheit und setzt aus diesem Grund eine Beeinflussung der Entscheidungsfähigkeit von Verbrauchern i. S. d. \3 Abs. 2 UWG voraus. ${ }^{870} \mathrm{Im}$ Gegensatz zu $\int 5 \mathrm{a}$ Abs. $1 \mathrm{UWG}$ stellt $\int 5 \mathrm{a}$ Abs. $2 \mathrm{UWG}$ aber nicht auf das Verschweigen einer entscheidungsrelevanten Tatsache ab, sondern auf das Vorenthalten ${ }^{871}$ einer wesentlichen Information. ${ }^{872}$ Die Einordnung einer Information als wesentlich i. S. d. \5a Abs. 2 UWG stellt somit den Dreh- und Angelpunkt bei der Beurteilung der Unlauterkeit einer geschäftlichen Handlung gegenüber

${ }^{868}$ Erwägungsgrund 14 der UGP-Richtlinie.

${ }^{869}$ A. A. Pfeifer in: Fezer, UWG, $\int 5$ a, Rn. 36, der seine Ansicht damit begründet, dass die Gesetzesbegründung ausführt, dass die Richtlinie ,eine nicht abschließende Liste von Informationen “" enthält (Regierungsentwurf und Begründung zum UWG vom 20.08.2008, BT.-Drs. 16/10145, S. 25). Diese Aussage in der Gesetzesbegründung bezieht sich aber allein auf die Liste in $\int 5 \mathrm{a}$ Abs. 3 UWG. Zudem wollte der Gesetzgeber damit keine zusätzlichen wesentlichen Kennzeichen für bestimmte Produkte aufnehmen, sondern allein Art. 7 Abs. 4 UGP-Richtlinie umsetzen, vgl. Regierungsentwurf und Begründung zum UWG vom 20.08.2008, BT.-Drs. 16/10145, S. 25 f.

870 Dieser Verweis auf $₫ 3$ Abs. 2 UWG setzt die in der UGP-Richtlinie enthaltenen zusätzlichen Voraussetzungen neben der Vorenthaltung einer wesentlichen Information zwar nicht eins-zueins um, jedoch können diese vollständig aus $₫ 3$ Abs. 2 UWG sowie $₫ 3$ Abs. 1 UWG im Hinblick auf die Relevanzklausel herausgelesen werden, so auch der Regierungsentwurf und Begründung zum UWG vom 20.08.2008, BT.-Drs. 16/10145, S. 17 f., S. 25 f.

871 Unter den Begriff des Vorenthaltens fällt dabei nach Ansicht des historischen Gesetzgebers auch das Verheimlichen von Informationen, die unklare, unverständliche, zweideutige oder verspätete Bereitstellung von Informationen sowie die fehlenden Kenntlichmachung des kommerziellen Zwecks des geschäftlichen Handlung, so dass diese in Art. 7 Abs. 2 UGP-Richtlinie ausdrücklich benannten Verhaltensweisen keine Aufnahme in das UWG gefunden haben, Regierungsentwurf und Begründung zum UWG vom 20.08.2008, BT.-Drs. 16/10145, S. 25.

872 „Unlauter handelt, wer die Entscheidungsfreibeit von Verbrauchern $\{. .$.$\} dadurch beeinflusst, dass er eine Infor-$ mation vorenthält, die $\{\ldots\}$ wesentlich ist", $₫ 5$ a Abs. 2 UWG. 
Verbrauchern aufgrund eines Unterlassens dar. ${ }^{873}$ Erst im Anschluss daran stellt sich die Frage, ob diese dem Verbraucher i. S. d. UWG vorenthalten wurde und welche Relevanz das Vorenthalten der wesentlichen Information für die Entscheidung des Verbrauchers hatte. ${ }^{874}$ Dabei muss jedoch berücksichtigt werden, dass auch die Übermittlung von wesentlichen Informationen aufgrund der allgemeinen Umstände und des verwendeten Kommunikationsmittels nur eingeschränkt möglich sein kann. Scheitert die Übermittlung einer wesentlichen Information hieran, geht dies zu Lasten des von der geschäftlichen Handlung betroffenen Verbrauchers. ${ }^{875}$ Zudem scheidet eine Irreführung durch Unterlassen immer dann aus, ,wenn sich die betreffenden Tatsachen bereits unmittelbar aus den Umständen ergeben." ${ }^{\prime 876}$

Entsprechend ist zunächst zu untersuchen, ob die Informationen über die Bindung oder Nichtbindung an einen Verhaltenskodex beziehungsweise den Verstoß gegen einen Verhaltenskodex wesentlich i. S. d. S 5a Abs. 2 UWG sind. Dies wäre unter anderem dann der Fall, wenn diese Informationen von Absatz 3 und 4 erfasst würden. Denn die in diesen benannten Informationen sind, wie von Art. 7 Abs. 4 und 5 UGP-Richtlinie vorgeben, als wesentlich anzusehen. ${ }^{877}$ Aber auch nicht von den Absätzen 3 und 4 erfasste Informationen können wesentlich sein, da diese keine abschließende Regelung darstellen. ${ }^{878}$ In diesem Fall muss sich die Wesentlichkeit direkt aus der Grundregelung des $\int 5 \mathrm{a}$ Abs. 2 UWG ergeben.

\section{Wesentlichkeit der Informationen}

a. Wesentlichkeit nach \5a Abs. 3 UWG

Die nach $\int 5 \mathrm{a}$ Abs. $3 \mathrm{UWG}$ als wesentlich geltenden Informationen werden entgegen der amtlichen Begründung zum Regierungsentwurf ${ }^{879}$ abschließend durch die aus den Nummern 1 bis 5 bestehende Auflistung bestimmt. ${ }^{880}$ Dies ergibt sich aus der richtlinienkonformen Auslegung der Norm, da eine solche Erweiterungsmöglichkeit nicht mit dem Wortlaut von Art. 7 Abs. 4 UGP-Richtlinie vereinbar ist. Denn diesem sind keinerlei Hinweise darauf zu entnehmen, dass die Richtlini-

\footnotetext{
873 Zum Verhältnis der Tatbestandsmerkmale „wesentliche Information“ und „Vorenthalten“ Dreyer in: Harte-Bavendamm/Henning-Bodewig, UWG, \5a, Rn. 29.

${ }^{874}$ Zur Notwendigkeit der Unterscheidung zwischen Wesentlichkeit und dem Vorenthalten einer Information, welche sich aus $\int 5 \mathrm{a}$ Abs. 2 UWG im Gegensatz zur Gesamtkonzeption von Art. 7 UGP-Richtlinie nicht ergibt, Dreyer in: Harte-Bavendamm/Henning-Bodewig, UWG, $\int 5 \mathrm{a}, \mathrm{Rn}$. $29 \mathrm{ff}$.

875 Dreyer in: Harte-Bavendamm/Henning-Bodewig, UWG, \5a, Rn. 38 ff.

876 Regierungsentwurf und Begründung zum UWG vom 20.08.2008, BT.-Drs. 16/10145, S. 25.

877 Regierungsentwurf und Begründung zum UWG vom 20.08.2008, BT.-Drs. 16/10145, S. 18.

878 Dreyer in: Harte-Bavendamm/Henning-Bodewig, UWG, \5a, Rn. 56.

879 Regierungsentwurf und Begründung zum UWG vom 22.08.2004, BT.-Drs. 15/1487, S. 25 f.

880 Dreyer in: Harte-Bavendamm/Henning-Bodewig, UWG, \5a, Rn. 56.
} 
engeber die genannten Informationen nur als Beispiele benennen wollte. Vielmehr spricht gerade das Fehlen einer entsprechenden Formulierung für den abschlieBenden Charakter der Auflistung. Dies hatte der deutsche Gesetzgeber auch anerkannt, da er feststellte, dass sich eine Erweiterungsfähigkeit der Auflistung nicht aus Art. 7 Abs. 4 UGP-Richtlinie ergibt. ${ }^{881}$ Entgegen der vom deutschen Gesetzgeber angeführten Begründung ergibt sich die Erweiterungsfähigkeit des Kataloges aus $\int 5 \mathrm{a}$ Abs. 3 UWG aber auch nicht daraus, dass Art. 7 Abs. 5 UGPRichtlinie ausdrücklich keine abschließende Regelung trifft. ${ }^{882}$ Denn die Ansatzpunkte für die Einordnung einer Information als wesentlich unterscheiden sich bei den Absätzen 4 und 5 von Art. 7 UGP-Richtlinie so erheblich, dass entgegen der Ansicht des deutschen Gesetzgebers eine unterschiedliche Beurteilung nicht nur anders sein kann, sondern vielmehr sein muss. Denn während Art. 7 Abs. 4 UGP-Richtlinie selbst eine Auswahl von Informationen trifft, die als wesentlich einzuordnen sind, verweist Art. 7 Abs. 5 UGP-Richtlinie auf im Gemeinschaftsrecht festgelegte Informationsanforderungen. Dass diese nur im Rahmen einer nicht erschöpfenden Liste aufgeführt werden ist bereits dadurch gerechtfertigt, dass andernfalls nach Erlass der UGP-Richtlinie festgelegte Informationsanforderungen nicht automatisch ebenfalls als wesentlich anzusehen wären, sondern zunächst eine Änderung der UGP-Richtlinie notwendig wäre. Hinzu kommt, dass die sich aus dem Gemeinschaftsrecht ergebenden Informationsanforderungen auch ohne abschließende Auflistung ermitteln lassen und die Verantwortlichen einer geschäftlichen Handlung somit eindeutig wissen, welche Informationen sie zur Verfügung stellen müssen. Dies wäre bei einer nicht abschließenden Auflistung in $\int 5 \mathrm{a}$ Abs. 3 UWG nicht der Fall. Insofern spricht gerade dieser Unterschied dafür, dass die Richtliniengeber im Rahmen von Art. 7 Abs. 4 UGPRichtlinie bewusst auf eine solche Formulierung verzichtet haben, während sie in Absatz 5 eine solche verwendet haben.

Der Begrenzung der unwiderlegbaren Wesentlichkeitsvermutung des \5a Abs. 3 UWG auf die in den Nummern 1 bis 5 genannten Informationen steht auch nicht entgegen, dass die Richtliniengeber mit der Regelung Basisinformationen festlegen wollte, die der Verbraucher für eine informierte geschäftliche Entscheidung benötigt. ${ }^{883}$ Denn auch wenn das Wort Basisinformationen so ausgelegt werden kann ${ }^{884}$, dass neben diesen noch weitere Informationen wesentlich sind, ergibt sich doch aus der Gesamtbetrachtung, dass die Richtliniengeber für die Fallgruppe der Aufforderung zum Kauf die unwiderlegbare Vermutung der Wesentlichkeit nach

\footnotetext{
${ }^{881}$ Regierungsentwurf und Begründung zum UWG vom 22.08.2004, BT.-Drs. 15/1487, S. 26. Dort heißt es: „Dies (die Einordnung der Auflistung als nicht abschließend, Anmerkung d Verf.) ergibt sich zwar nicht unmittelbar aus Artikel 7 Abs. 4 der Ricbtlinie."

882 Regierungsentwurf und Begründung zum UWG vom 22.08.2004, BT.-Drs. 15/1487, S. 26.

883 Erwägungsgrund 14 der UGP-Richtlinie.

884 Sosnitza in: Piper/Ohly/Sosnitza, UWG, \5a, Rn. 22.
} 
\5a Abs. 3 UWG nur für bestimmte Informationen, nämlich die ausdrücklich benannten, festlegen wollten. 885 Insofern ist der Begriff der Basisinformationen dahingehend zu verstehen, dass genau diese Informationen immer als Basis der geschäftlichen Entscheidung vorliegen müssen. Dies bedeutet jedoch nicht, dass für die Fallgruppe der Aufforderung zum Kauf nur die $\ 5$ a Abs. 3 UWG benannten Informationen wesentlich sein können ${ }^{886}$, sondern nur, dass nur die dort benannten Informationen als wesentlich gelten. Darüber hinaus kann sich die Wesentlichkeit anderer Informationen natürlich aus $₫ 5 \mathrm{a} A b s .2 \mathrm{UWG}$ ergeben.

\section{aa. Erfasste geschäftliche Handlungen}

Die von $₫ 5 \mathrm{a}$ Abs. 3 UWG erfassten Informationen gelten nicht für alle geschäftlichen Handlungen ${ }^{887}$ gegenüber Verbrauchern als wesentlich, sondern nur für solche, in denen „Waren oder Dienstleistungen unter Hinweis auf deren Merkmale und Preis in einer dem vervvendeten Kommunikationsmittel angemessenen Weise so angeboten (werden, Ergänzung d. d. Verf.), dass ein durchschnittlicher Verbraucher das Geschäft abschließen kann." "888 Der deutsche Gesetzgeber hat sich bezüglich dieser Begrenzung des sachlichen Anwendungsbereiches ${ }^{889}$ bewusst gegen die sprachlich prägnantere Formulierung einer Aufforderung zum Kauf aus der UGP-Richtlinie entschieden. Er hat deren Legaldefinition aus Art. 2 lit. i) UGP-Richtlinie stattdessen direkt in den Gesetzestext integriert ${ }^{890}$, um terminologische Abgrenzungsprobleme mit dem deutschen Recht zu vermeiden. ${ }^{891}$ Denn nach den deutschen Rechtsbegrifflichkeiten wären von der Formulierung der UGP-Richtlinie nur Kaufverträge nach

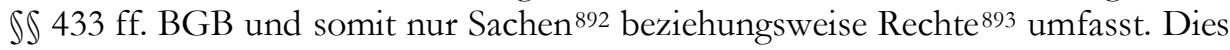
würde jedoch den Anwendungsbereich gegenüber der UGP-Richtlinie beschränken, was mit der durch die UGP-Richtlinie angestrebten Vollharmonisierung nicht zu vereinbaren wäre. Denn eine Aufforderung zum Kauf betrifft nach Art. 2 lit. i) UGP-Richtlinie, jede kommerzielle Kommunikation, die die Merkmale des Produkts $\{. .$. angibt", wobei der Begriff des Produkts i. S. d. UGP-Richtlinie wiederum neben Waren auch Dienstleistungen umfasst. ${ }^{894}$

885 Erwägungsgrund 14 der UGP-Richtlinie.

${ }^{886}$ Urteil des EuGH v. 12.05.2011 C-122/10 Ving Sverige AB, Tz. 24, A. A. Köhler, WRP 2009, S. 109, 116, der $\ 5$ a Abs. 3 UWG als ,in doppelter Hinsicbt abschließender Natur" einordnet.

887 Eine solche Beschränkung auf bestimmte geschäftliche Handlungen findet sich auch im Rahmen der Irreführung aufgrund einer Verwechslungsgefahr, vgl. zu dieser Kapitel 6B.IV.1.

888 \5a Abs. 3 UWG.

889 Vgl. Kapitel 4A.

890 Art. 2 lit. i) UGP-Richtlinie: ,„,Aufforderung zum Kauf"jede kommerzielle Kommunikation, die die Merkmale des Produkts und den Preis in einer Weise angibt, die den Mitteln der verwendeten kommerziellen Kommunikation angemessen ist und den Verbraucher dadurch in die Lage versetzt, einen Kauf zu tätigen;"

${ }^{891}$ Regierungsentwurf und Begründung zum UWG vom 20.08.2008, BT.-Drs. 16/10145, S. 25.

$892 \int 433$ Abs. 1 BGB.

$893 \$ 453$ BGB.

894 Art. 2 lit. c) UGP-Richtlinie. 
Grundvoraussetzung eines Waren- oder Dienstleistungsangebotes gemäß \5a Abs. 3 UWG ist somit entsprechend der Legaldefinition der Aufforderung zum Kauf in Art. 2 lit. i) UGP-Richtlinie, dass es eine kommerzielle Kommunikation ${ }^{895}$ darstellt. Insofern wird der grundsätzliche weite sachliche Anwendungsbereich des UWG auf geschäftliche Handlungen 896 im Rahmen von \5a Abs. 3 UWG merklich eingeschränkt. So stellen beispielsweise die Mitteilung auf der Homepage eines Unternehmens oder eine Pressemitteilung über die Abgabe eines Verhaltenskodexes keine kommerzielle Kommunikation dar. ${ }^{897}$ Aber auch nicht jede kommerzielle Kommunikation stellt eine Aufforderung zum Kauf dar. ${ }^{898}$ Denn eine solche liegt nur vor, wenn die kommerzielle Kommunikation über die wesentlichen Merkmale des Produkts und seines Preises informiert. ${ }^{899}$ Somit kann die reine Image- oder Aufmerksamkeitswerbung keine Aufforderung zum Kauf darstellen ${ }^{900}$, da sie kein konkretes Produkt bewirbt und somit auch nicht über dessen Merkmale aufklären kann. Insoweit unterliegt auch die Werbung eines Unternehmens, welche sich auf das Verhalten eines Unternehmens oder seinen Verhaltenskodex beschränkt, nicht den Informationspflichten nach $\int 5 \mathrm{a}$ Abs. 2 i. V.m. Abs. 3 UWG. 901 Aber auch innerhalb der verbleibenden konkreten Produktwerbung reicht es nicht aus, dass ein Produkt und seine jeweiligen Vorzüge beworben werden, um als Aufforderung zum Kauf eingeordnet zu werden. Denn Art. 2 lit. i) UGP-Richtlinie setzt ergänzend voraus, dass die kommerzielle Kommunikation Angaben zum Preis der Ware oder Dienstleistung enthält und „den Verbraucher in die Lage versetət, den Kauf zu tätigen". ${ }^{902}$ Die geschäftliche Handlung muss somit wohl jedenfalls ${ }^{903}$ die essentialia negotii des abzuschließenden Geschäfts enthalten ${ }^{904}$, damit ein Durchschnittverbraucher das Angebot unmittelbar annehmen kann. ${ }^{905}$ Insofern scheidet auch ein nicht unerheblicher, wenn nicht sogar ein Großteil der Produktwerbungen aus. ${ }^{906}$ Nämlich nahezu alle Produktwerbungen, die unmittelbar von den Herstellern der Waren geschaltet werden. Denn in diesen wird in aller Regel nur dann ein Preis genannt, wenn der Hersteller

\footnotetext{
895 Zum Begriff der kommerziellen Kommunikation vgl. Kapitel 4A.II.1.

896 Vgl. Kapitel 4A.

${ }^{897}$ Vgl. Kapitel 4A.

898 Erwägungsgrund 14 der UGP-Richtlinie.

899 Art. 2 lit. i) UGP-Richtlinie.

900 Regierungsentwurf und Begründung zum UWG vom 20.08.2008, BT.-Drs. 16/10145, S. 25, Bornkamm in: Köhler/Bornkamm, UWG, \5a, Rn. 30c, Wiebe, JBl. 2007, 67, 76.

901 Und somit die als Nr. 3 dargestellt geschäftliche Handlung im Zusammenhang mit Verhaltenskodizes unter Kapitel 4A..

902 Der deutsche Gesetzgeber hat dies unter der Voraussetzung, „,dass ein durchschnittlicher Verbraucher das Geschäft abschließen kann" in \5a Abs. 3 UWG übernommen.

903 Wiebe, JBl. 2007, 69, 76.

904 Bornkamm in: Köhler/Bornkamm, UWG, \ 5a, Rn. 30a, Veelken, WRP, 1, 22.

905 Regierungsentwurf und Begründung zum UWG vom 20.08.2008, BT.-Drs. 16/10145, S. 25.

906 Vgl. Fn. 904.
} 
über ein eigenes Händlernetz verfügt. ${ }^{907}$ Somit verbleiben im Bereich der Werbung als klassischem Anwendungsbereich des UWG im Wesentlichen nur die Werbemaßnahmen von Händlern. Darüber hinaus sind die in $\ 5$ a Abs. 3 UWG genannten Informationen aber natürlich auch im Rahmen von individuellen Absatzförderungsmaßnahmen wesentlich.

bb. Informationen im Zusammenhang mit Verhaltenskodizes als wesentliche Information gemäß $\ 5 \mathrm{a}$ Abs. $3 \mathrm{UWG}$

Für die Einordnung von Informationen im Zusammenhang mit Verhaltenskodizes wie die bestehende oder nichtbestehende Bindung oder den Verstoß gegen einen solchen als wesentlich, müssten diese Informationen unter einen der Umstände aus der Auflistung in $\ 5 \mathrm{a}$ Abs. 3 UWG gefasst werden können. Konkret handelt es sich hierbei um die wesentlichen Merkmale einer Ware oder Dienstleistung (Nr. 1), Informationen zum handelnden Unternehmer (Nr. 2), zum Preis beziehungsweise zur Preisberechnung (Nr. 3), zu Zahlungs-, Liefer- und Leistungsbedingungen sowie zu Verfahren im Umgang mit Beschwerden (Nr. 4) und über Rücktritts- oder Widerrufsrechte (Nr. 5). Da es sich bei den Information, ob ein Unternehmen einen Verhaltenskodex unterzeichnet oder gegen diesen verstoBen hat, um unternehmensbezogene Angaben handelt ${ }^{908}$, müsste sich ihre Wesentlichkeit somit aus $\ 5$ a Abs. 3 Nr. 2 UWG ergeben. Dieser erkennt aber nach seinem Wortlaut nur "die Identität und Anschrift des Unternehmers, gegebenenfalls die Identität und Anschrift des Unternebmers, für den er handelt" als wesentliche unternehmensbezogene Informationen an, nicht aber, ob ein Unternehmen einen Verhaltenskodex unterzeichnet oder gegen diesen verstoßen hat.

Jedoch wird im Hinblick auf die Einordnung unter $\int 5 \mathrm{a}$ Abs. 3 UWG vertreten, dass es sich bei CSR-Kriterien um eine wesentliche Information gemäß $\ 5 \mathrm{a}$ Abs. 3 Nr. 1 UWG handelt, wobei CSR-Kriterien als Unternehmensstandards bestimmt werden, ,welche die soziale und ethische Verantwortung der Unternehmen konkretisieren. "909 \5a Abs. 3 Nr. 1 UWG bestimmt in Umsetzung ${ }^{910}$ von Art. 7 Abs. 4 lit. a) UGP-Richtlinie ${ }^{911}$, alle wesentlichen Merkmale der (angebotenen, Ergänzung d. d. Verf.) Ware oder Dienstleistung" als wesentliche Informationen. ${ }^{912}$ Was als wesent-

\footnotetext{
907 Vgl. Fn. 904, so beispielsweise bei Automobilherstellern, die in Werbungen regelmäßig den Einstiegspreis (,ab EUR XXX“) abgeben.

908 Vgl. Kapitel 6B.I.

909 Puchinger, S. 99 ff.

910 Dabei wurde wie im Rahmen der gesamten Umsetzung lediglich der Begriff des Produktes aus der UGP-Richtlinie durch die Begriffe Ware und Dienstleistung ersetzt, vgl. zum Hintergrund Kapitel 4A.II.2.

911 Regierungsentwurf und Begründung zum UWG vom 20.08.2008, BT.-Drs. 16/10145, S. 26.

912 Entscheidend für die Feststellung der Wesentlichkeit einer Information für die Entscheidung eines Verbrauchers über den Erwerb einer Ware oder Dienstleistung ist somit, ob die Information ein wesentliches Merkmal der angebotenen Ware oder Dienstleistung betrifft.
} 
liches Merkmal einer Ware oder Dienstleistung i. S. d. \5a Abs. 3 Nr. 1 UWG gilt, ist somit aufgrund des Gebots der richtlinienkonformen Auslegung durch die Feststellung dessen zu ermitteln, was nach Art. 7 Abs. 4 lit. a) UGP-Richtlinie als wesentliches Merkmal eines Produktes gilt. Nach Ansicht von Puchinger kann hierfür ohne Einschränkung auf $\ 5$ Abs. 1 Satz 2 Nr. 1 UWG zurückgegriffen werden, so dass die dort genannten Beispiele „Verfügbarkeit, Art, Ausführung, Vorteile, Risiken, Zusammensetzung, Zubebör, Verfahren oder Zeitpunkt der Herstellung, Lieferung oder Erbringung, Zwecktauglichkeit, Verwendungsmöglichkeit, Menge, Beschaffenheit, Kundendienst und Beschwerdeverfahren, geographische oder betriebliche Herkunft, von der Verwendung zu erwartende Ergebnisse oder die Engebnisse oder wesentlichen Bestandteile von Tests der Waren oder Dienstleistungen" allesamt auch gemäß $\int 5 \mathrm{a}$ Abs. $3 \mathrm{Nr}$. 1 UWG als wesentliche Merkmale einer Ware oder Dienstleistung anzusehen wären.913 Dem ist jedoch bereits aufgrund des Gebots der richtlinienkonformen Auslegung insoweit entgegenzutreten, als die in $\int 5$ Abs. 1 Satz 2 Nr. 1 UWG benannten Beispiele nicht vollständig identisch sind mit den in Art. 6 Abs. 1 lit. b) UGP-Richtlinie benannten Beispielen für wesentliche Merkmale von Produkten. Denn der deutsche Gesetzgeber hat in seinem $\ 5$ Abs. 1 Satz 2 Nr. 1 UWG die lit. a) und b) des Art. 6 Abs. 1 UGP-Richtlinie zusammengefasst. Insofern ist die Art des Produkts kein wesentliches Merkmal i. S. d. Art. 6 Abs. 1 lit. b) UGP-Richtlinie. Unabhängig von dieser Nuance ist jedoch der vollständigen Gleichstellung der wesentlichen Merkmale eines Produktes nach den Art. 6 Abs. 1 lit. b) und Art. 7 Abs. 4 lit. a) UGP-Richtlinie auch im Grundsatz entgegenzutreten. Denn die lange und nicht abschließende - Beispielliste der wesentlichen Merkmale in Art. 6 Abs. 1 lit. b) UGP-Richtlinie, welche die Einschränkung auf wesentliche Merkmale in gewisser Weise ad absurdum führt, da offensichtlich alles wesentlich sein kann, ${ }^{914}$ mag zwar im Rahmen der Irreführung durch aktives Handeln angemessen sein, nicht jedoch im Rahmen der Irreführung durch Unterlassen. So ist nicht jedes Merkmal, über das theoretisch irregeführt werden kann, im Rahmen einer Aufforderung zum Kauf auch erforderlich, um eine informierte geschäftliche Entscheidung zu treffen. ${ }^{915}$ Für eine unterschiedliche Auslegung des Begriffs der wesentlichen Merkmale spricht zudem, dass die Richtliniengeber bei Art. 7 Abs. 4 lit. a) UGP-Richtlinie auf eine Bezugnahme auf Art. 6 Abs. 1 lit. b) UGPRichtlinie beziehungsweise eine Übernahme der dort angeführten Beispiele verzichtet haben. Darüber hinaus zeigt ein Blick in den ursprünglichen Richtlinienvorschlag, dass nur Informationen über die „wichtigsten Merkmale" eines Produkts wesentlich sein sollten. ${ }^{916}$ Die mit dem Gemeinsamen Standpunkt eingeführte Änderung des Wortlautes in wesentliche Merkmale kann aufgrund der vielfältigen

\footnotetext{
913 Puchinger, S. 92.

914 Micklitz in: Münchner Kommentar zum Lauterkeitsrecht, EG F, Rn. 180.

915 Pfeifer in: Fezer, UWG, \5a, Rn. 42.

916 Art. 7 Abs. 3 lit. a) des Vorschlags für eine UGP-Richtlinie, KOM (2003) 356 endgültig.
} 
Verwendung des Begriffs der Wesentlichkeit in der UGP-Richtlinie und dem Fehlen jeglicher Begründung für diese Änderung nicht als bewusste Maßnahme angesehen werden, um eine inhaltliche Angleichung mit Art. 6 Abs. 1 lit. b) UGPRichtlinie herbeizuführen. ${ }^{917}$ Was i. S. d. Art. 7 Abs. 4 lit. a) UGP-Richtlinie und somit auch i. S. d. \5a Abs. 3 Nr. 1 UWG als wesentliches Produktmerkmal anzusehen ist, muss daher eigenständig und schutzbezogen ermittelt werden. ${ }^{918}$ Hierbei muss insbesondere Berücksichtigung finden, welche Informationen ein Verbraucher, der kurz vor einem Vertragsschluss steht ${ }^{919}$, über eine Ware oder Dienstleistung benötigt. Köhler schlägt hierzu vor, Art. 7 Abs. 4 UGP-Richtlinie im Lichte der an die berufliche Sorgfalt aus Art. 2 lit. h) UGP-Richtlinie ${ }^{920}$ gestellten Anforderungen auszulegen. ${ }^{921}$ Dies würde bedeuten, dass solche Informationen nicht vorenthalten werden dürften, ,bei denen billigerweise davon ausgegangen werden kann, dass der Gewerbetreibende sie gegenüber dem Verbraucher gemäß den anständigen Mark.tgepflogenheiten und/oder dem allgemeinen Grundsatz von Treu und Glauben in seinem Tätigkeitsbereich" angibt.

Im Hinblick auf die hier wesentliche Fragestellung, ob die Bindung an einen Verhaltenskodex ein wesentliches Merkmal einer Ware oder Dienstleistung i. S. d. \5a Abs. 3 Nr. 1 UWG darstellt, ist es jedoch unerheblich, ob der Katalog des $\int 5$ Abs. 1 Satz 2 Nr. 1 UWG zur Bestimmung der wesentlichen Merkmale i. S. d. $\int 5 \mathrm{a}$ Abs. 3 Nr. 1 UWG herangezogen werden kann oder ob die nach $\int 5$ a Abs. 3 Nr. 1 UWG wesentlichen Merkmale einer Ware oder Dienstleistung unabhängig von diesem eigenständig ermittelt werden müssen. Denn das Bestehen oder Nichtbestehen eines Verhaltenskodexes in einem Unternehmen stellt als solches überhaupt kein Merkmal der Waren oder Dienstleistungen des Unternehmens dar, sondern eine Information über das Unternehmen. ${ }^{922}$ So lässt sich aus der Information, dass ein Unternehmen einen Verhaltenskodex unterzeichnet hat, kein allgemeingültiger Hinweis darauf entnehmen, welche Verpflichtungen der Verhaltenskodex enthält und ob diese wiederum als Merkmal der Produkte oder Dienstleistungen des Unternehmens anzusehen sind.923 Aus diesem Grund kann aber auch der Verstoß gegen einen Verhaltenskodex kein wesentliches Merkmal einer Ware oder Dienstleistung sein. Bei genauer Betrachtung steht dies auch nicht im Widerspruch zu Puchinger, da diese nicht auf die Information abstellt, dass ein Unternehmen einen Verhaltenskodex unterzeichnet hat beziehungsweise sich auf sonstige Weise im Rahmen der CSR engagiert, sondern auf die dabei oftmals

\footnotetext{
917 A. A.: Dreyer in: Harte-Bavendamm/Henning-Bodewig, UWG, \5a, Rn. 58.

918 Pfeifer in: Fezer, UWG, S 5a, Rn. 42.

${ }^{919}$ Da $\int 5 a$ Abs. 3 Nr. 1 UWG nur diese Situation betrifft, vgl. Kapitel 6C.II.1.a.aa.

920 Dieser Begriff wurde im UWG als fachliche Sorgfalt umdeklariert (vgl. nachfolgend Kapitel 6D.I) und in $\int 2$ Abs. 1 Nr. 7 UWG definiert.

921 Köhler, WRP 2009, 109, 116.

922 Vgl. Kapitel 6B.I.

923 Vgl. zu den Gründen Kapitel 6B.I.
} 
enthaltenen Verpflichtungen zu einem Verzicht auf Kinderarbeit oder auf gesundheitsgefährdende Produktionsbedingungen. ${ }^{924}$ Diese konkreten Verpflichtungen — und nicht die Information über die Unterzeichnung eines Verhaltenskodexes oder das sonstige Engagement eines Unternehmens im Bereich CSR - ordnet Puchinger als wesentliche Merkmale einer Ware oder Dienstleistung i. S. d. S 5a Abs. 3 Nr. 1 UWG ein. ${ }^{925}$ Ob dies der Fall ist oder ob mit Köhler nur solche Umstände als wesentliche Merkmale einer Ware oder Dienstleistung i. S. d. S $5 \mathrm{a}$ Abs. 3 Nr. 1 UWG anzuerkennen sind, die mit der Qualität und Brauchbarkeit einer Ware oder Dienstleistung zu tun haben, nicht aber deren Vertriebs- und Herstellungsbedingungen, ${ }^{926}$ ist im Hinblick auf die hier untersuchte Informationspflicht über die bestehende Bindung an einen Verhaltenskodex nicht relevant und wird daher nicht vertieft untersucht. Der Verfasser neigt jedoch dazu, sich der Ansicht von Köbler anzuschließen, da dessen Beschränkung auf Qualität und Preis einer Ware als entscheidungsrelevante Kriterien sich mit dem Ergebnis von Untersuchungen des Instituts für Markt-Umwelt-Gesellschaft e. V. an der Universität Hannover deckt. ${ }^{927}$ Dieses hat in einer repräsentativen Haushaltsbefragung ${ }^{928}$ ermittelt, dass zwar 60 Prozent der Befragten ,ein latentes Interesse an CSRInformationen" haben ${ }^{929}$, allerdings nur für 21 Prozent der Befragten ein hoher Bedarf für CSR-Informationen besteht. ${ }^{930}$ Zudem hat dieses Institut im Rahmen einer weiteren Untersuchung ermittelt, dass 100 Prozent der Befragten bei den konkret ausgewählten Produkten Joggingschuhe und Waschmaschine die CSR des Anbieters nachrangig im Vergleich zu Produktqualität und zum Preis gewichten. ${ }^{931}$

Darüber hinaus spricht auch die Gesetzgebungshistorie dagegen, die Bindung an einen Verhaltenskodex als wesentliche Information i. S. d. Art. 7 Abs. 4 UGPRichtlinie einzuordnen. Denn das Europäische Parlament hatte vorgeschlagen, die

\footnotetext{
924 Puchinger, S. $99 \mathrm{ff}$.

925 Puchinger, S. 99 ff.

926 Köhler, WRP 2009, 109, 116, zustimmt hierzu Sosnitza in: Piper/Ohly, Sosnitza, UWG, \5a, Rn. 26.

927 Auch Puchinger, S. 99, dort Fn. 353, stützt sich auf diese Untersuchungen des imug, entnimmt diesen jedoch konträre Schlussfolgerungen.

928 Welche allerdings bereits im Februar 2006 durchgeführt wurde.

${ }^{929}$ Ergebnisse der repräsentativen Haushaltsbefragung des imug „CSR-Informationsbedarf von Verbrauchern - Informations- und Einkaufsverbalten im Kontext von CSR“, S. 16, abrufbar unter http://www.imug.de/pdfs/verbraucher/hp_imug_CSR_Verbraucher_Repraesentativbefragung _2006_03_16.pdf, zuletzt abgerufen am 29.04.2013. Auf diesen Wert bezieht sich Puchinger, S. 99, dort Fn. 353, allerdings unter Verzicht auf die Einschränkung, dass das Interesse nur latent besteht.

${ }^{930}$ Ergebnisse der repräsentativen Haushaltsbefragung des imug „CSR-Informationsbedarf von Verbrauchern - Informations- und Einkaufsverhalten im Kontext von CSR", S. 8, S. 32.

931 Imug Arbeitspapier 17/2007, Corporate Social Responisibility als Verbraucherinformation, S. 16, dort Fn. 12.
} 
in diesem enthaltende Auflistung der wesentlichen Informationen unter anderem insoweit zu erweitern, dass, gegebenenfalls die Beteiligung an einem Verbaltenskodex“" als wesentliche Information einzuordnen ist. ${ }^{932}$ Der Verzicht auf eine entsprechende Erweiterung der Auflistung muss damit auch als Zurückweisung der Begründung des Europäischen Parlamentes angesehen werden, welches mit dieser Ergänzung sicherstellen wollte, „dass die Verbraucher klar und ordnungsgemäß unterrichtet werden.“933

b. Wesentlichkeit nach $₫ 5$ a Abs. 4 UWG

Als wesentlich nach $\int 5 a$ Abs. 4 UWG gelten alle „Informationen, die dem Verbraucher auf Grund gemeinschaftsrechtlicher Verordnungen oder nach Rechtsvorschriften zur Umsetzung gemeinschaftsrechtlicher Richtlinien für kommerzielle Kommunikation einschließlich Werbung und Marketing nicht vorenthalten werden dürfen." Informationen im Zusammenhang mit Verhaltenskodizes wie die bestehende oder nichtbestehende Bindung oder der Verstoß gegen einen solchen gelten somit als wesentlich i. S. d. \5a Abs. 4 UWG, wenn das europäische Recht entsprechende Informationsanforderungen bei einer kommerziellen Kommunikation vorsieht.

Während weder die in Anhang II der UGP-Richtlinie benannten Normen aus vierzehn europäischen Richtlinien noch andere europäische Rechtsakte Informationspflichten bezüglich der Nichteinhaltung eines Verhaltenskodexes oder dem Nichtbestehen eines Verhaltenskodexes enthalten, sieht Art. 6 Abs. 1 lit. n.) Verbraucherrechte-Richtlinie ${ }^{934}$ für im Fernabsatz oder außerhalb geschlossener Geschäftsräume geschlossene Verträge eine Informationspflicht über bestehende Verhaltenskodizes sowie darüber, wie man Exemplare von diesem Verhaltenskodex erhalten kann, vor. ${ }^{935}$

Da die Gespräche bezüglich des konkreten Abschlusses eines Vertrages eine kommerzielle Kommunikation darstellen ${ }^{936}$, dürfen Unternehmen, die einen Verhaltenskodex i. S. d. $\ 2$ Abs. 1 Nr. 5 UWG abgegeben haben, diese Information Verbrauchern nicht vorenthalten.

\footnotetext{
932 Standpunkt des Europäischen Parlaments, P5_TC1-COD(2003)0134.

933 Begründung zum Änderungsantrag 53 des Entwurfs einer legislativen Entschließung des Europäischen Parlamentes zum Vorschlag der Kommission für die UGP-Richtlinie, A5-0188/2004 endgültig.

934 Richtlinie 2011/83/EU des Europäischen Parlaments und des Rates vom 25. Oktober 2011.

935 Wird eine entsprechende Information unterlassen, ergibt sich die Unlauterkeit der jeweiligen geschäftlichen Handlung zudem direkt aus dem Rechtsbruchtatbestand des $₫ 4 \mathrm{Nr}$. 11 UWG, Köhler, NJW 2008, 3032, 3034, vgl. zu \ 4 Nr. 11 UWG, vgl Kapitel 6A.III.

936 Zum Begriff der kommerziellen Kommunikation vgl. 1. Kapitel 4A.II.1.
} 
c. Wesentlichkeit nach $\int 5$ a Abs. 2 UWG

Auch Informationen, die nicht von den Absätzen 3 und 4 erfasst werden, können wesentliche Informationen i. S. d. \5a Abs. 2 UWG sein. ${ }^{937}$ Denn die Absätze 3 und 4 bestimmten nicht abschließend, welche Informationen wesentlich sind ${ }^{938}$, sondern enthalten nur eine unwiderlegbare gesetzliche Vermutung für die Wesentlichkeit der von ihnen erfassten Informationen. ${ }^{939}$ Allerdings enthält das UWG weder die Definition einer wesentlichen Information noch sonstige Anhaltspunkte zur Bestimmung einer Information als wesentlich. Aus Art. 7 Abs. 1 UGPRichtlinie lässt sich jedoch entnehmen, dass es sich bei wesentlichen Informationen um solche handelt, „die der durchschnittliche Verbraucher je nach den Umständen benötigt, um eine informierte geschäftliche Entscheidung zu treffen". ${ }^{940}$ Diese Schlussfolgerung aus dem Wortlaut von Art. 7 Abs. 1 UGP-Richtlinie stellt weder eine Überstrapazierung desselben dar noch wird durch sie das Merkmal der „Wesentlichkeit überflüssig. ${ }^{941}$ Vielmehr handelt es sich um eine Begriffserklärung, welche entgegen der Systematik der UGP-Richtlinie nicht bei den Definitionen in Art. 2 UGPRichtlinie aufgeführt ist, sondern direkt im Anschluss an den unbestimmten Rechtsbegriff. Problematisch ist aber die Begriffserklärung selbst, da sie unbestimmte Rechtsbegriffe verwendet, die wiederum selbst der Auslegung bedürfen. So definiert die UGP-Richtlinie zwar der Begriff der geschäftlichen Entscheidung ${ }^{942}$, enthält aber keine Festlegungen darüber, welche Anforderungen an eine informierte geschäftliche Entscheidung zu stellen sind, obwohl sie auch im Rahmen der Definition der ,wesentlichen Beeinflussung des wirtschaftlichen Verhaltens des Verbraucher" auf eine solche abstellt.943 Den Erwägungsgründen der UGP-Richtlinie lässt sich im Bezug auf informierte geschäftliche Entscheidungen nur entnehmen, dass die Richtlinie nach dem Willen der Richtliniengeber auch solche „Praktiken \{...\} umfasst, die den Verbraucher durch Täuschung davon abhalten, eine informierte und damit effektive Wabl zu treffen. "944 Diese bringt jedoch ebenfalls keinen Mehrwert, da nur eine Verlagerung auf einen weiteren unbestimmten Rechtsbegriff erfolgt.

Diese Unbestimmtheit der wesentlichen Begrifflichkeiten ${ }^{945}$ ist jedoch insoweit gewollt und unvermeidlich, da durch $\int 5$ a Abs. 2 UWG, anders als durch die Ab-

\footnotetext{
${ }^{937}$ Dreyer in: Harte-Bavendamm/Henning-Bodewig, UWG, \5a, Rn. 56.

938 A.A. Köhler, NJW 2008, 3032, 3034.

939 Dreyer in: Harte-Bavendamm/Henning-Bodewig, UWG, \ 5a, Rn. 32.

940 Diese Schlussfolgerung zieht auch Dreyer in: Harte-Bavendamm/Henning-Bodewig, UWG, \5a, Rn. 35.

941 A. A.: Köhler, WRP 2009, S. 109, 116.

942 Art. 2 lit. k) UGP-Richtlinie.

943 Zwar stellt die Definition der „wesentlichen Beeinflussung des wirtschaftlichen Verbaltens des Verbraucher" in Art. 2 lit. e) UGP-Richtlinie auf die informierte Entscheidung des Verbrauchers ab, jedoch ist hiermit keine inhaltliche Unterscheidung verbunden.

944 Erwägungsgrund 14 der UGP-Richtlinie.

945 Wesentliche Information und informierte geschäftliche Entscheidung.
} 
sätze 3 und 4, nicht bestimmte Informationen als in jedem Falle wesentlich eingeordnet werden sollen. So sind nach dem Willen der Richtliniengeber selbst so grundlegende Informationen aus Art. 7 Abs. 4 UGP-Richtlinie ${ }^{946}$ wie der Preis einer Ware oder Dienstleistung oder die Identität des handelnden Unternehmers nicht bei allen geschäftlichen Handlungen grundsätzlich als wesentlich anzusehen. Wörtlich heißt es hierzu in Erwägungsgrund 14 der UGP-Richtlinie: „Solche Informationen (die in Art. 7 Abs. 4 UGP-Richtlinie genannten, Anmerkung d. Verf.) müssen nicht notwendigerweise in jeder Werbung enthalten sein." Wenn aber bereits so grundlegende Informationen wie der Preis einer Ware oder Dienstleistung oder der Identität des handelnden Unternehmers nicht zwangsläufig notwendig sind, um eine informierte Entscheidung zu treffen, so kann dies erst Recht nicht für die Informationen, ob eine Bindung an einen Verhaltenskodex besteht oder gegen einen solchen Verstoßen wurde, angenommen werden. ${ }^{947}$

Vielmehr ist zur Ermittlung der Wesentlichkeit einer Information aufgrund von \5a Abs. 2 UWG immer eine Interessensabwägung unter Berücksichtigung des Leitbildes vom informierten, verständigen und angemessen aufmerksamen Durchschnittsverbrauchers ${ }^{948}$ vorzunehmen ${ }^{949}$, bei welcher alle tatsächlichen Umstände Beachtung finden. ${ }^{950}$ Für diese Interessensabwägung lässt sich aus der Bezugnahme der UGP-Richtlinie zur Bestimmung der Wesentlichkeit einer Information auf ihre Notwendigkeit für eine informierte geschäftliche Entscheidung der Grundsatz entnehmen, dass die Informationsanforderungen um so mehr steigen, je enger der Zusammenhang der zu bewertenden geschäftlichen Handlung mit der konkreten geschäftlichen Entscheidung eines Verbrauchers ist. ${ }^{951}$ Dies steht auch nicht im Widerspruch zur Einordnung der Auflistung in $\int 5 \mathrm{a}$ Abs. $3 \mathrm{UWG}$ als abschließend. ${ }^{952}$ Denn diese schließt nicht aus, dass in diesem nicht genannte Informationen als wesentlich gelten können, sondern verwehrt anderen Informationen allein die für die Informationen aus $\int 5 \mathrm{a}$ Abs. 3 UWG geltende unwiderlegbare gesetzliche Wesentlichkeitsvermutung. Andererseits führt diese Maßgabe dazu, dass Image- oder Aufmerksamkeitswerbung nur in absoluten Ausnahmefällen aufgrund des Vorenthaltens von Informationen unlauter sind. Dies ist jedoch angemessen, da der verständige, aufmerksame und durchschnittlich informierte Verbraucher erkennt, dass es Unternehmen bei diesen darum geht, ihr Ansehen zu verbessern beziehungsweise seine Aufmerksamkeit zu erlangen, nicht aber ihn

\footnotetext{
946 Umgesetzt in $\int 5$ a Abs. 3 UWG, vgl. Kapitel 6C.II.1.

947 A. A. Fezer, WRP 2009, S. 577, 584 f., WRP 2007, 1021, 1029 f., der hierfür jedoch ein allgemeines Informationsgebot zugrunde legt.

948 Regierungsentwurf und Begründung zum UWG vom 20.08.2008, BT.-Drs. 16/10145, S. 22.

949 Nordemann, Wettbewerbsrecht Markenrecht, S. 240, Rn. 383.

950 Art. 7 Abs. 1 UGP-Richtlinie.

951 Micklitz in: Münchner Kommentar zum Lauterkeitsrecht, EG F, Rn. 199.

952 Vgl. Kapitel 6C.II.1.a.
} 
konkret zu einem Vertragsschluss zu bewegen. ${ }^{953}$ Entsprechend scheidet die Annahme der Unlauterkeit aufgrund $\int 5 \mathrm{a}$ Abs. 2 UWG für in dieser Beziehung noch unter der Image- oder Aufmerksamkeitswerbung anzusiedelnde geschäftliche Handlungen wie beispielsweise eine Pressemitteilung nahezu aus. ${ }^{954}$ Aber auch für näher am Vertragsschluss liegenden geschäftliche Handlungen wie beispielsweise die Produktwerbung muss im Rahmen der Interessensabwägung ein besonderer Umstand hinzukommen, damit die Informationen, ob ein Unternehmer einen Verhaltenskodex unterzeichnet oder gegen einen Verhaltenskodex verstoßen hat, als wesentlich i. S. d. \5a Abs. 2 UWG eingeordnet werden können. In Bezug auf diese Informationen im Zusammenhang mit Verhaltenskodizes stellt es dabei einen zu beachtenden tatsächlichen Umstand dar, ob und in welcher Art und Weise der Unternehmer zuvor auf seine Bindung an einen Verhaltenskodex hingewiesen beziehungsweise einen besonderen Vertrauenstatbestand dahingehend geschaffen hat, dass er seinen Verhaltenskodex zu jeder Zeit einhält. Dabei kann zur Bestimmung, wann ein solcher besonderer Vertrauenstatbestand besteht, auf die für $\int 5 \mathrm{a}$ Abs. 1 UWG entwickelten Maßgaben zurückgegriffen werden. ${ }^{955}$ Dabei ist jedoch ebenfalls zu beachten, dass CSR-Informationen nur bei einem geringen Teil der Verbraucher ein hoher Stellenwert bei ihren geschäftlichen Entscheidungen zukommt, während sie die geschäftlichen Entscheidungen des Großteils der Verbraucher nicht beeinflussen. ${ }^{956}$ Zudem ist im Rahmen der vorzunehmenden Interessenabwägung zu berücksichtigten, dass die Einschränkung auf das Vorenthalten von wesentlichen Informationen für eine Irreführung durch Unterlassen nicht nur dem Informationsbedarf der Verbraucher Rechnung tragen sollte, sondern ,dem Umstand, dass ein Übermaß an Information genauso ein Problem darstellen kann wie unzureichende Angaben."957 Hierzu hat das Institut für Markt-UmweltGesellschaft e. V. an der Universität Hannover es als offensichtlich bezeichnet, „dass Kaufentscheidungen, bei denen sowohl die Produkte (Preise und Qualität) als auch die Unternehmen (sozial-ökologische Verantwortung) geachtet wird, nicht unbedingt einfacher werden."958

953 Dreyer in: Harte-Bavendamm/Henning-Bodewig, UWG, \5a, Rn. 35.

954 Vgl. die Auflistung der geschäftlichen Handlungen im Zusammenhang mit Verhaltenskodizes in Kapitel 4A.

955 Vgl. Kapitel 6C.I.

956 Vgl. Kapitel 6C.II.1.a.bb unter Bezugnahme auf die Ergebnisse der repräsentativen Haushaltsbefragung des imug „CSR-Informationsbedarf von Verbrauchern — Informations- und Einkaufsverhalten im Kontext von CSR“, S. 8, S. 32.

957 Erwägungsgrund 65 des Vorschlages der Kommission für die UGP-Richtlinie KOM (2003) 0356 endgültig.

958 Imug Arbeitspapier 17/2007, Corporate Social Responisibility als Verbraucherinformation, S. 16. 


\section{2. $\quad$ Vorenthalten einer wesentlichen Information}

Der Begriff des Vorenthaltens umfasst nach Ansicht des deutschen Gesetzgebers auch das Verheimlichen von Informationen, das unklare, unverständliche, zweideutige und nicht rechtzeitige Bereitstellen von Informationen sowie die Nichtkenntlichmachung des kommerziellen Zwecks einer geschäftlichen Handlung. ${ }^{959}$ Der Begriff umfasst dementsprechend aus sich selbst heraus die in Art. 7 Abs. 2 UGP-Richtlinie benannten Verhaltensweisen, so dass der Gesetzgeber für diese keinen eigenen Umsetzungsbedarf erkannte. ${ }^{960}$ Dabei setzen alle diese vom Begriff des Vorenthaltens erfassten Verhaltensweisen voraus, dass der Vorenthaltende im Besitz der jeweiligen Information ist. ${ }^{961}$ Dies schließt eine Verpflichtung des Unternehmers aus, sich vom Verbraucher erbetene Informationen zu beschaffen. ${ }^{962}$ Dies ist im Zusammenhang mit Verhaltenskodizes relevant, wenn Waren von Dritten angeboten oder deren Dienstleistungen vermittelt werden. So muss ein (großes) Handelsunternehmen sich nicht für jedes Produkt in seinem Sortiment darüber informieren, ob dessen Hersteller einen Verhaltenskodex unterzeichnet hat, geschweige denn, ob gegen diesen verstoßen wurde. Gleichfalls erlangen auch Hersteller von Waren oftmals nicht unmittelbar Kenntnis von einem Verstoß gegen ihren Verhaltenskodex, da die hierfür notwendigen Monitoringmaßnahmen regelmäßig nur in bestimmten Zeitabständen durchgeführt werden. Problematisch ist dies insbesondere dann, wenn die Produktion der Waren, wie bei Konsumgütern oftmals üblich, von Subunternehmern übernommen wird.

Für die Annahme eines Vorenthaltens i. S. d. $\int 5$ a Abs. 2 UWG ist aber auch die Beschränkungen des für die geschäftliche Handlung verwendeten Kommunikationsmittels zu berücksichtigen, so dass nicht jedes Unterlassen der Angabe wesentlicher Informationen ausreicht. ${ }^{963}$ Diese Einschränkung trägt „dem Umstand Rechnung, dass die Möglichkeiten zur Vermittlung von Informationen in räumlicher oder zeitlicher Hinsicht beschränkt sein können."964 Dabei unterliegen die Medien Fernsehen und Rundfunk sowohl räumlichen als auch zeitlichen Beschränkungen, während gedruckte Medien im Regelfall nur räumlichen Beschränkungen unterliegen. ${ }^{965} \mathrm{Je}$ doch kann in Einzelfällen auch bei gedruckten Medien eine zeitliche Begrenzung bestehen. So beispielsweise bei Plakatwerbung an Autobahnen, für welche nur ein eingeschränkter Wahrnehmungszeitraum besteht. Werden der geschäftlichen Handlung dabei durch das verwendete Kommunikationsmittel entsprechende

\footnotetext{
${ }^{959}$ Regierungsentwurf und Begründung zum UWG vom 20.08.2008, BT.-Drs. 16/10145, S. 25.

960 Regierungsentwurf und Begründung zum UWG vom 20.08.2008, BT.-Drs. 16/10145, S. 25.

961 Köhler, WRP 2009, 109, 116, Sosnitza in: Piper/Ohly/Sosnitza, UWG, \5a, Rn. 6.

962 Köhler, WRP 2009, 109, 116, Sosnitza in: Piper/Ohly/Sosnitza, UWG, \ 5a, Rn. 6.

963 Dreyer in: Harte-Bavendamm/Henning-Bodewig, UWG, \5a, Rn. 38.

964 Regierungsentwurf und Begründung zum UWG vom 20.08.2008, BT.-Drs. 16/10145, S. 25 in Übernahme von Art. 7 Abs. 3 UGP-Richtlinie.

965 Pfeifer in: Fezer, UWG, \5a, Rn. 33 f.
} 
räumliche oder zeitliche Beschränkungen auferlegt, so werden nicht nur diese Beschränkungen in die Interessenabwägung einbezogen, sondern auch alle Maßnahmen, die der Unternehmers veranlasst, um den Verbrauchern die Information anderweitig zur Verfügung zu stellen. ${ }^{966}$ Dies ist \5a Abs. 2 UWG zwar nicht direkt zu entnehmen, jedoch ist der Begriff des Vorenthaltens aufgrund der sogenannten Medienklausel in Art. 7 Abs. 3 UGP-Richtlinie entsprechend auszulegen. ${ }^{967}$ Eine solche anderweitige Mitteilung ist jedoch nur dann als Ersatz für eine unmittelbare Information im Rahmen der geschäftlichen Handlung anzuerkennen, wenn sich in dieser ein deutlich erkennbarer Hinweis auf die Stelle enthält, an welcher der wesentlichen Informationen zur Verfügung gestellt sind. ${ }^{968}$ Denkbar ist hier zum Beispiel der Verweis auf eine Webseite. ${ }^{969}$

\section{Zusammenfassung}

Die Bedeutung der Irreführung durch Unterlassen im Zusammenhang mit Verhaltenskodizes ist im Hinblick auf geschäftliche Handlungen im b2b-Verhältnis trotz der Einführung des $\int 5 \mathrm{a} U \mathrm{UWG}$ weiterhin gering. Grund hierfür ist, dass für diese nicht die zur Umsetzung der Vorgaben der UGP-Richtlinie neu eingeführten Absätze 2 bis 4 des $\int 5 a$ UWG gelten, sondern nur der nun als $\int 5 a$ Abs. 1 UWG bezeichnete $\int 5$ Abs. 2 Satz 2 UWG 2004.

Somit gilt für geschäftliche Handlungen im b2b-Verhältnis weiterhin, dass ein irreführendes Unterlassen nur dann in Betracht kommt, wenn für den Verantwortlichen der geschäftlichen Handlung eine entsprechende Aufklärungspflicht besteht. Eine solche wird für die Tatsache, ob ein Unternehmen an einen Verhaltenskodex gebunden ist oder gegen diesen verstoßen hat, jedoch nur in Ausnahmefällen vorliegen. Beispielsweise wenn eine solche Aufklärungspflicht vertraglich vereinbart wurde oder das Vorverhalten des Verantwortlichen der geschäftlichen Handlung eine entsprechende Aufklärung erforderlich macht.

Dahingegen hat die Irreführung durch Unterlassen im Zusammenhang mit Verhaltenskodizes bei geschäftlichen Handlungen im b2c-Bereich durch die Neueinführung insofern an Bedeutung gewonnen, als zumindest bei im Fernabsatz oder außerhalb geschlossener Geschäftsräume geschlossenen Verträgen das Bestehen eines Verhaltenskodexes eine wesentliche Information gemäß \5a Abs. 4 UWG i. V. m. Art. 6 Abs. 1 lit. n.) Verbraucherrechterichtlinie ist, die einem Verbraucher nicht vorenthalten werden darf.

Abseits von im Fernabsatz oder außerhalb geschlossener Geschäftsräume geschlossener Verträge stellt das Bestehen und Nichtbestehen eines Verhaltensko-

\footnotetext{
966 Art. 7 Abs. 3 UGP-Richtlinie.

967 Dreyer in: Harte-Bavendamm/Henning-Bodewig, UWG, \5a, Rn. 40.

968 Pfeifer in: Fezer, UWG, $\ 5$ a, Rn. 35.

969 Urteil des EuGH v. 12.05.2011 C-122/10 Ving Sverige AB, Tz. 24.
} 
dexes beziehungsweise die Einhaltung eines Verhaltenskodexes jedoch auch im b2c-Bereich keine wesentliche Information i. S. d. \$5a UWG dar. Insbesondere stellen diese Informationen kein wesentliches Merkmal einer Ware oder Dienstleistung nach $\int 5 \mathrm{a}$ Abs. 3 Nr. 1 UWG dar. Auch wenn einzelne oftmals in Verhaltenskodizes enthaltene Verpflichtungen wie beispielsweise der Verzicht auf Kinderarbeit oder der Verzicht auf Pflanzenschutzmittel möglicherweise als wesentliche Merkmale von Waren und Dienstleistungen einzuordnen sind, so gilt dies nicht für die das gesellschaftliche Engagement von Unternehmen im Allgemeinen, unter welches auch Verhaltenskodizes zu fassen sind. Gleichfalls ergibt sich die Wesentlichkeit dieser Informationen in diesem Bereich nicht direkt aus $\int 5 \mathrm{a}$ Abs. 2 UWG. Denn entsprechende Informationen haben nur für einen geringen Teil der Verbraucher einen für die Annahme der Wesentlichkeit notwendigen hohen Stellenwert. Insofern kann nicht davon ausgegangen werden kann, dass der durchschnittliche Verbraucher diese Informationen benötigt, um eine von Art. 7 Abs. 1 UGP-Richtlinie vorausgesetzte informierte geschäftliche Entscheidung zu treffen.

\section{Unlauterkeit beziehungsweise Unzulässigkeit von Verstößen gegen Verhaltenskodizes gemäß der Generalklauseln aus $\mathbb{} 3$ Abs. 1 und Abs. 2 Satz 1 UWG}

Die Unlauterkeit beziehungsweise Unzulässigkeit einer geschäftlichen Handlung kann sich nicht nur aus den Beispielsfällen der $\$ \int 4 \mathrm{ff}$. UWG, sondern auch unmittelbar aufgrund der Generalklauseln aus $₫ 3$ Abs. 1 und Abs. 2 Satz 1 UWG ergeben. Allerdings ist zu beachten, dass die Generalklauseln nicht ohne weiteres als Auffangtatbestände verwendet werden können, wenn eine geschäftliche Handlung die Voraussetzungen eines der Beispieltatbestände des UWG nicht erfüllt.970 Denn andernfalls würden die mit der Kodifizierung dieser Beispielsfälle verbundenen Wertungen des Gesetzgebers untergraben, da auch das Nichterfüllen der Tatbestandsvoraussetzungen Ausdruck dieser Wertungen sein kann. ${ }^{971}$ So ist im Hinblick auf die Unlauterkeit von Verstößen gegen Verpflichtungen aus Verhaltenskodizes zu berücksichtigen, dass entsprechende Verhaltensweisen durch

\footnotetext{
970 Urteil des BGH v. 09.09.2010 - I ZR 157/08 (FSA-Kodex) (OLG München) in GRUR 2011, 431, 432, Köhler in: Köhler/Bornkamm, UWG, \ 4, Rn. 0.6, a. A. Sack, WRP 2005, 531, 532, der $\$ 3$ Abs. 1 UWG weiterhin eine ,umfassende lückenfüllende Funktion“" zuspricht.

971 Urteil des OLG Frankfurt/Main v. 04.08.2005 - 6 U 224/04 in GRUR 2005, 1064, 1066, welches auch unter Geltung des UWG 2008 durch das Urteil des BGH v. 17.07.2008 - I ZR 160/05 (OLG Frankfurt/M) (Lion-Schokoriegel) in GRUR 2009, 71 ff. bestätigt wurde, Köhler in: Köhler/Bornkamm, UWG, $\int 4$, Rn. 0.6.
} 
$\int 5$ Abs. 1 Satz 1 i. V. m. $\int 5$ Abs. 1 Satz 2 Nr. 6 UWG geregelt werden und der Gesetzgeber beziehungsweise die Richtliniengeber besondere Voraussetzungen für die Irreführung (und damit die Unlauterkeit) entsprechender geschäftlicher Handlungen vorausgesetzt haben. ${ }^{972}$ Allerdings werden durch $\int 5$ Abs. 1 Satz 1 i. V. m. $\int 5$ Abs. 1 Satz 2 Nr. 6 UWG nur Sachverhalte geregelt, in welchen derjenige, der gegen die Verpflichtungen aus einem Verhaltenskodex verstoßen hat, sich selbst auf diesen verpflichtet hat. Insoweit besteht eine Regelungslücke, welche die Anwendung der Generalklauseln rechtfertigt. ${ }^{973}$ Diese betrifft die Frage, inwieweit Verhaltenskodizes allgemein zur Bestimmung des lauteren Verhaltens beitragen. Hierbei ist jedoch zu beachten, dass ein Rückgriff auf die Generalklausel nur dann in Betracht kommt, wenn das mit der zu bewertenden geschäftlichen Handlung verbundene Verhalten im Hinblick auf seinen Unlauterkeitsgehalt den Beispielsfällen des UWG entspricht. ${ }^{974}$

\section{Verhältnis der Generalklauseln}

Das UWG enthält seit seiner Reformierung zur Umsetzung der UGP-Richtlinie im Jahr 2008 zwei Generalklauseln. Erstens die gegenüber dem UWG 2004 nur leicht angepasste ${ }^{975}$ Generalklausel in $\ 3$ Abs. 1 UWG, welche unlautere geschäftliche Handlungen für unzulässig erklärt, soweit sie die in der Norm festgeschriebene Relevanzschwelle überschreiten, und zweitens die zur Umsetzung von Art. 5 Abs. 2 Satz 1 UGP-Richtlinie aufgenommene sogenannte Verbrauchergeneralklausel ${ }^{976}$ in $₫ 3$ Abs. 2 Satz 1 UWG. Letztere erklärt unter Verzicht auf das Merkmal der Unlauterkeit geschäftliche Handlungen direkt für unzulässig, die nicht der anzuwendenden fachlichen Sorgfalt entsprechen, soweit ebenfalls die in der Norm enthaltene Relevanzschwelle überschritten ist. Dieses Nebeneinander von zwei Generalklauseln hat zu einer heftigen Kontroverse in der Literatur über die Bedeutung von $₫ 3$ Abs. 2 S. 1 UWG und das Verhältnis der beiden Generalklauseln geführt. 977

Bei genauerer Betrachtung lösen sich die auf den ersten Blick zwischen den beiden Generalklauseln bestehenden Unterschiede jedoch weitestgehend auf, da die Verbrauchergeneralklausel letztlich lediglich unter ausführlicher Beschreibung

\footnotetext{
972 Vgl. zu diesen besonderen Voraussetzungen 0.

973 Köhler in: Köhler/Bornkamm, UWG, \ 4, Rn. 0.6.

974 Urteil des BGH v. 09.09.2010 - I ZR 157/08 (FSA-Kodex) (OLG München) in GRUR 2011, 431, 432, Schünemann in: Harte-Bavendamm/Henning-Bodewig, UWG, $₫ 3$, Rn. $54 \mathrm{ff}$.

975 Das im UWG 2004 verwendete Merkmal der „Beeinträchtigung des Wettbewerbs zum Nacbteil von Marktteilnebmern“ wurde durch das Merkmal der „spürbaren Beeinträcbtigung der Interessen von Mitbewerbern, Verbrauchern und sonstigen Marktteilnehmern" ersetzt, vgl. Regierungsentwurf und Begründung zum UWG vom 20.08.2008, BT.-Drs. 16/10145, S. 22.

976 Für viele: Beater, Rn. 1114, Scherer, WRP 2010, 586.

977 Eine gute Darstellung der vertretenen Positionen gibt Scherer, WRP 2010, 586, 587 f.
} 
der Tatbestandsmerkmale des $\int 3$ Abs. 1 UWG dieselbe Rechtsfolge anordnet wie dieser. ${ }^{978}$ Denn der Gesetzgeber versteht ausweislich der Begründung der Novellierung des UWG im Jahr 2004, auf welche er in seiner Begründung zur Reformierung des UWG im Jahr 2008 ausdrücklich Bezug genommen hat ${ }^{979}$, solche Handlungen als unlauter, ,die den anständigen Gepflogenheiten in Handel, Gewerbe und Handwerk oder selbständiger beruflicher Tätigkeit zuwiderlaufen. "980 Wesentlich für die Erfüllung des Tatbestandsmerkmales der Unlauterkeit aus \ 3 Abs. 1 UWG ist somit ein Verstoß gegen die anständigen Gepflogenheiten. Gleiches gilt für \3 Abs. 2 Satz 1 UWG. Zwar stellt dieser als wesentliches Tatbestandsmerkmal auf einen Verstoß gegen die fachliche Sorgfalt ab, jedoch handelt es sich hierbei lediglich um einen sprachlichen, nicht aber um einen inhaltlichen Unterschied. Denn die Definition der fachlichen Sorgfalt als der „Standard an Fachkenntnissen und Sorgfalt, von dem billigerweise angenommen werden kann, dass ein Unternehmer ibn in seinem Tätigkeitsbereich gegenüber Verbrauchern nach Treu und Glauben unter Berücksichtigung der Marktgepflogenheiten einhält ${ }^{* 981}$ stellt letztlich, neben dem allgemeinen Grundsatz von Treu und Glauben, ebenfalls im Wesentlichen auf die anständigen Marktgepflogenheiten ab. Dies ergibt sich aus der Definition des Begriffs der beruflichen Sorgfalt in Art. 2 lit. h) UGP-Richtlinie982, welcher laut dem deutschen Gesetzgeber dem Begriff der fachlichen Sorgfalt entspricht. ${ }^{983}$ Denn die berufliche Sorgfalt nach Art. 2 lit. h) UGP-Richtlinie bestimmt sich zuerst nach Maßgaben der anständigen Marktgepflogenheiten und erst im Anschluss daran nach dem allgemeinen Grundsatz von Treu und Glauben.

Auch die im Hinblick auf die jeweils zweite Tatbestandsvoraussetzung der Relevanz bestehenden Unterschiede der Generalklauseln ${ }^{984}$ führen inhaltlich nicht zu unterschiedlichen Ergebnissen. So setzten beide Generalklauseln einheitlich die

978 Scherer, WRP 2010, 586, 592.

${ }^{979}$ Regierungsentwurf und Begründung zum UWG vom 20.08.2008, BT.-Drs. 16/10145, S. 15.

980 Regierungsentwurf und Begründung zum UWG vom 22.08.2004, BT.-Drs. 15/1487, S. 16.

$981 § 2$ Abs. 1 Nr. 7 UWG.

982 Art. 2 lit. h) UGP-Richtlinie: ,,, berufliche Sorgfalt" der Standard an Fachkenntnissen und Sorgfalt, bei denen billigerweise davon ausgegangen werden kann, dass der Gewerbetreibende sie gegenüber dem Verbraucher gemäß den anständigen Mark.tgepflogenheiten und/oder dem allgemeinen Grundsatz,von Treu und Glauben in seinem Tätigkeitsbereich anwendet."

983 Inhaltlich wurde lediglich der Begriff der beruflichen Sorgfalt aus der UGP-Richtlinie durch den Begriff der fachlichen Sorgfalt ersetzt. Dies war notwendig, da Berufe nach deutschem Rechtsverständnis nur von natürlichen Personen ausgeübt werden könne, der Anwendungsbereich jedoch auch juristische Personen umfassen sollte, Regierungsentwurf und Begründung zum UWG vom 20.08.2008, BT.-Drs. 16/10145, S. 22 f.

984 So verlangt $\int 3$ Abs. 1 UWG, dass die geschäftliche Handlung geeignet ist, die Interessen von Mitbewerbern, Verbrauchern oder sonstigen Marktteilnehmern spürbar zu beeinträchtigen, während \ 3 Abs. 2 Satz 1 UWG voraussetzt, dass die geschäftliche Handlung geeignet ist, die Fähigkeit des Verbrauchers, sich auf Grund von Informationen zu entscheiden, spürbar zu beeinträchtigen und den Verbraucher damit zu einer geschäftlichen Entscheidung zu veranlassen, die er andernfalls nicht getroffen hätte. 
Eignung der unlauteren geschäftlichen Handlung beziehungsweise der gegen die fachliche Sorgfalt verstoßenden geschäftlichen Handlung zur spürbaren Beeinträchtigung voraus. Auch wenn die Verbrauchergeneralklausel diese spürbare Beeinträchtigung konkret auf die Fähigkeit des Verbrauchers bezieht, sich aufgrund von Informationen zu entscheiden und kumulativ voraussetzt, dass die geschäftliche Handlung einen Durchschnittsverbraucher zu einer geschäftlichen Entscheidung veranlasst, stellt dies keinen inhaltlichen Unterschied zur Generalklausel des $₫ 3$ Abs. 1 UWG dar, welche allgemein auf die Interessen der Mitbewerber, Verbraucher und sonstigen Marktteilnehmer abstellt. Denn der deutsche Gesetzgeber hat den Begriff der Spürbarkeit gewählt, weil die UGP-Richtlinie diesen in der Definition der wesentlichen Beeinflussung des wirtschaftlichen Verbaltens des Verbrauchers ${ }^{985}$ verwendet ${ }^{986}$, auf welche wiederum die UGP-Richtlinie in der Ursprungsnorm der Verbrauchergeneralklauseln ${ }^{987}$ abstellt. ${ }^{988}$ Der deutsche Gesetzgeber wollte somit die damit verbundenen Vorgaben auf den von ihm verwendeten Begriff der spürbaren Beeinträchtigung übertragen.

Auch wenn ein solches Nebeneinander von zwei Regelungen, die jeweils für weitestgehend identische Sachverhalte die gleiche Rechtsfolge anordnen, sicherlich kein Paradebeispiel der Kunst der Gesetzgebung darstellt, ist die Regelung des $₫ 3$ Abs. 2 Satz 1 UWG entgegen der Ansicht von Scherer nicht überflüssigg ${ }^{989}$, soweit darunter ersatzlos verzichtbar zu verstehen ist. Denn dass mit der Einfügung von $\int 3$ Abs. 2 Satz 1 UWG keine wesentliche Änderung der Rechtslage verbunden sein würde, war auch dem Gesetzgeber bewusst. ${ }^{990}$ Dieser hat sich jedoch bewusst für eine Parallelregelung entschieden, da er den mit Art. 3 Abs. 2 Satz 1 UWG umgesetzten Art. 5 Abs. 2 UGP-Richtlinie als ein so wesentliches Element des Richtlinienkonzeptes erachtete, für welchen ein Bedürfnis zur Klarstellung gegenüber Marktteilnehmern aus anderen Mitgliedstaaten bestehe. ${ }^{991}$ Gerade vor diesem Hintergrund erscheint es jedoch unverständlich, warum der Gesetzgeber die Regelung des Art. 5 Abs. 2 lit. a) UGP-Richtlinie nicht als Legaldefinition der Unlau-

\footnotetext{
985 Art. 2 lit. e) UGP-Richtlinie: „,,wesentliche Beeinflussung des wirtschaftlichen Verbaltens des Verbrauchers “ die Anwendung einer Geschäftspraxis, um die Fähigkeit des Verbrauchers, eine informierte Entscheidung zu treffen, spürbar zu beeinträchtigen und damit den Verbraucher zu einer geschäftlichen Entscheidung zu veranlassen, die er andernfalls nicht getroffen bätte;"

986 Regierungsentwurf und Begründung zum UWG vom 20.08.2008, BT.-Drs. 16/10145, S. 22.

987 Der deutsche Gesetzgeber hat dahingegen in $\$ 3$ Abs. 2 Satz 1 UWG nicht nur Art. 5 Abs. 2 lit. a) und b) UGP-Richtlinie aufgenommen, sondern auch die Definition der ,wesentliche Beeinflussung des wirtschaftlichen Verbaltens des Verbrauchers" aus Art. 2 lit. e) UGP-Richtlinie (vgl. zu dessen Inhalt Fn. 985), Regierungsentwurf und Begründung zum UWG vom 20.08.2008, BT.-Drs. 16/10145, S. 22.
}

988 Art. 5 Abs. 2 lit. b) UGP-Richtlinie.

989 Scherer, WRP 2010, 586, 592.

990 Regierungsentwurf und Begründung zum UWG vom 20.08.2008, BT.-Drs. 16/10145, S. 15.

991 Vgl. Fn. 990. 
terkeit ${ }^{992}$ umgesetzt hat. Dies hätte nicht nur die Klarstellungswirkung für Marktteilnehmer aus anderen Mitgliedstaaten verstärkt, sondern auch zu einer erheblichen Verbesserung der Verständlichkeit des UWG im Allgemeinen beigetragen. Denn schließlich handelt es sich beim Begriff der Unlauterkeit um einen der zwei zentralen Begriffe des UWG ${ }^{993}$, dessen Definition im Gesetz selbst besser aufgehoben wäre als in der Gesetzesbegründung. ${ }^{994}$

II. Bedeutung von Verstößen gegen Verhaltenskodizes im Rahmen der Generalklauseln

\section{Ansatz der Rechtsprechung: Indizwirkung für Unlauterkeit}

Wie bereits unter Kapitel 2A dargestellt, hat der BGH bereits unter Geltung des UWG 1909 einen Automatismus (Verstoß gegen Wettbewerbsregeln beziehungsweise Wettbewerbsrichtlinien = Unlauterkeit) verneint, Wettbewerbsregeln beziehungsweise Wettbewerbsrichtlinien jedoch eine Indizwirkung bei der Beurteilung der Unlauterkeit von Verhaltensweisen zugestanden. ${ }^{995}$ Diese Indizwirkung wurde unter Geltung des UWG 2004 und der Änderung der Generalklausel ${ }^{996}$ zwar reduziert („allenfalls eine indizielle Bedeutung für die Frage der Unlauterkeit"), jedoch nicht vollständig aufgegeben. ${ }^{997} \mathrm{Da}$ mit der Änderung der Generalklausel in $\int 3$ Abs. 1 UWG und der Einführung der Verbrauchergeneralklausel in $₫ 3$ Abs. 2 Satz 1 UWG nach dem Willen und der Ansicht des deutschen Gesetzgebers keine Änderung der Rechtslage einhergehen sollte und musste ${ }^{998}$, stellt es keine Überraschung dar, dass der BGH unter Verweis auf die Probeabonnemententscheidung 999 und die dortige Begründung ${ }^{1000}$ diese, nun mehr ,(lediglich) indizielle Bedeutung“, auch unter dem UWG 2008 weiterhin anerkannt hat. ${ }^{1001}$ Dass der BGH aufgrund der Änderungen des UWG 2008 und der Einfügung verschiedener Regelungen zu Verhaltenskodizes keinen Anlass zur Änderung seiner Rechtsprechung gesehen hat, zeigt sich auch daran, dass er diese Rechtsfrage ausweislich des

\footnotetext{
992 So bezeichnet Schünemann die Regelung des Art. 5 Abs. 2 UGP-Richtlinie in HarteBavendamm/Henning-Bodewig, UWG, \&3, Rn. 5.

993 Neben dem Begriff der geschäftlichen Handlung, vgl. Kapitel 4A, dort Fn. 375.

994 Regierungsentwurf und Begründung zum UWG vom 22.08.2004, BT.-Drs. 15/1487, S. 16.

995 Urteil des BGH 08.11.1990 - I ZR 48/89 (OLG Hamm) (Wettbewerbsrichtlinie der Privatwirtschaft) in GRUR 1991, 462.

996 Vgl. Kapitel 2A.

997 Urteil des BGH v. 7. 2. 2006 - KZR 33/04 (OLG Hamburg) (Probeabonnement), in NJW 2006, 2627, 2629, Tz. 19.

998 Vgl. Fn. 990.

999 Urteil des BGH v. 7. 2. 2006 - I .ZR 33/04 (OLG Hamburg) (Probeabonnement) in NJW 2006, $2627 \mathrm{ff}$.

1000 Zur Begründung der Probeabonnement-Entscheidung vgl. Kapitel 2A.

1001 Urteil des BGH v. 09.09.2010 - I ZR 157/08 (OLG München) (FSA-Kodex) in GRUR 2011, 431, 432, Tz. 14.
} 
diesbezüglichen Einleitungssatzes in seinen Entscheidungsgründen für \ 3 UWG 2004 und \3 Abs. 1 UWG 2008 einheitlich beurteilt. ${ }^{1002}$ Allerdings hat der BGH bezüglich der konkreten Bedeutung auch festgestellt, dass „Regeln, die sich ein Verband oder ein sonstiger Zusammenschluss von Verkebrsbeteiligten gegeben hat, nur eine begrenəte Bedeutung" bei der Beurteilung der Unlauterkeit zugestanden werden kann. 1003

Über die bis dahin bestehende Rechtsprechung hinaus hat der BGH die bereits (lediglich) indizielle Bedeutung eines Verstoßes gegen eine selbstgesetzte Regel für die Annahme der Unlauterkeit nach $₫ 3$ Abs. 1 UWG jedoch dadurch weiter eingeschränkt, dass er selbst diese Indizwirkung nur dann zugesteht, „wenn sich die aus dem festgestellten Kodexverstoß abgeleitete Regelwidrigkeit des betreffenden Verbaltens gerade auch als eine wettbewerbsberogene, d.h. von den Schutzzwecken des Gesetzes gegen den unlauteren Wettbewerb (vgl. \1 UWG) erfasste Unzulässigkeit erweist." 1004 Zur Begründung verwies der BGH auf seine Zweckbetrieb-Entscheidung ${ }^{1005}$, in welcher er festgestellt hatte, dass es nicht die Aufgabe des Lauterkeitsrechtes sei, „alle nur denkbaren Regelverstöße im Zusammenhang mit geschäftlichen Handlungen auch lauterkeitsrechtlich zu sanktionieren." 1006 Auffällig ist, dass der BGH in diesem Zusammenhang den zum Zeitpunkt seiner FSA-Kodex-Entscheidung bereits im UWG definierten Begriff des Verhaltenskodexes vermeidet, obwohl der streitgegenständliche FSAKodex in den Kommentierungen des Urteils einstimmig als Verhaltenskodex eingeordnet wird ${ }^{1007}$ und auch der BGH selbst wohl von einer entsprechenden Einordnung ausgeht. ${ }^{1008}$ Grund hierfür dürfte sein, dass der BGH selbst diese begrenzte Bedeutung nicht sämtlichen Verhaltenskodizes i.S.d. $\int 5$ Abs. 1 Nr. 5 UWG zugesteht, sondern nur solchen mit einem gewissen Gewicht im Hinblick auf die Beschreibung der anständigen Gepflogenheiten wie beispielsweise Verhaltenskodizes von Verbänden.

Unabhängig von den Beweggründen des BGH, den Begriff des Verhaltenskodexes zu vermeiden, bedeutet diese zusätzliche Voraussetzung, dass ein Verstoß

1002 Urteil des BGH v. 09.09.2010 - I ZR 157/08 (OLG München) (FSA-Kodex) in GRUR 2011, 431, 432, Tz. 13.

1003 Urteil des BGH v. 09.09.2010 - I ZR 157/08 (OLG München) (FSA-Kodex) in GRUR 2011, 431, 432, Tz. 13.

1004 Urteil des BGH v. 09.09.2010 - I ZR 157/08 (OLG München) (FSA-Kodex) in GRUR 2011, 431, 432, Tz. 14.

1005 Urteil des BGH v. 02.12.2009 - I ZR 152/07 (OLG Stuttgart) (Zweckbetrieb) in GRUR 2010, 654, 657, Tz. 25.

1006 Urteil des BGH v. 09.09.2010 - I ZR 157/08 (OLG München) (FSA-Kodex) in GRUR 2011, 431, 432, Tz. 14.

1007 Fritzsche, LMK 2011, 316398, Nemeczek, GRUR 2011, 432 ff.

1008 Denn im darauffolgenden Absatz stellt der BGH fest, dass die UGP-Richtlinie im Hinblick auf die Bewertung der Unlauterkeit von Verstößen gegen Verhaltenskodizes keine andere Wertung enthält, Urteil des BGH v. 09.09.2010 - I ZR 157/08 (OLG München) (FSA-Kodex) in GRUR 2011, 431, 432, Tz. 15. 
gegen einen Verhaltenskodex nach der Rechtsprechung des BGH nur dann für die Unlauterkeit beziehungsweise Unzulässigkeit aufgrund der Generalklauseln relevant sein kann, wenn der Unrechtsgehalt des Verstoßes dem Unrechtsgehalt der Unlauterkeitstatbestände des UWG entspricht. Ausgeschlossen wären damit Verstöße gegen Verhaltenskodizes wie den in den „Leitlinien zur Umsetzung/Anwendung der Richtlinie 2005/29/EG über unlautere Geschäftspraktiken“" als Beispiel angeführten Verhaltenskodex über nachhaltige Holznutzung. ${ }^{1009}$ Denn die Verwendung von Holz aus Rodungsgebieten entspricht in ihrem Unrechtsgehalt keinem der Unlauterkeitstatbestände des UWG. ${ }^{1010}$ Gleiches dürfte für eine Vielzahl der Verpflichtungen in Verhaltenskodizes aus den Bereichen Ökologie, Ethik und soziale Mindeststandards gelten.

Verstößt eine geschäftliche Handlung gegen einen Verhaltenskodex und entspricht dieser Verstoß von seinem Unlauterkeitsgehalt den gesetzlichen Unlauterkeitstatbeständen, bedeutet dies jedoch nach der Rechtsprechung des BGH nicht zwangsläufig dessen Unlauterkeit. Vielmehr sieht der BGH Verhaltenskodizes lediglich als Mittel an, um festzustellen, „ob innerbalb der in Rede stehenden Verkehrskreise eine bestimmte tatsächliche Übung herrscht."1011 Selbst wenn eine entsprechende tatsächliche Übung angenommen werden muss, folgt aus dieser aber nicht zwangsläufig, dass die anständigen Gepflogenheiten in dieser Branche der in dem Verhaltenskodex dargestellten tatsächlichen Übung entsprechen. ${ }^{1012}$ Denn eine solche Gleichsetzung von tatsächlicher Übung und anständiger Gepflogenheiten würde das Übliche zur Norm erheben und dadurch den Wettbewerb in bedenklicher Weise beschränken. ${ }^{1013}$

Mit seinen Entscheidungen Probeabonnement und FSA-Kodex hat der BGH damit die Bedeutung von Verstößen gegen Verhaltenskodizes für die Unlauterkeit in einer solchen Art und Weise verkürzt, dass die weitere Verwendung des Begriffs der Indizwirkung nur noch als Reminiszenz zu werten ist. Denn mit seiner Entscheidung Wettbewerbsrichtlinie der Privatwirtschaft, welche die Indizwirkung begründete, hatte der BGH noch vorgegeben, dass, wenn eine Wettbewerbsrichtlinie ein Verhalten als unzulässig bezeichnet, zu prüfen ist, ob dies auch vom

${ }^{1009}$ Leitlinien zur Umsetzung der UGP-Richtlinie, (2009)1666, Punkt 2.5.6., S. 51 f., vgl. für das Beispiel Kapitel 3C.

1010 Unabhängig davon ist, dass natürlich die Angabe, ein Möbel sei aus entsprechendem Holz gefertigt, irreführend ist, wenn dies tatsächlich nicht der Fall ist.

1011 Urteil des BGH v. 09.09.2010 - I ZR 157/08 (OLG München) (FSA-Kodex) in GRUR 2011, 431, 432, Tz. 13.

1012 Urteil des BGH v. 09.09.2010 - I ZR 157/08 (OLG München) (FSA-Kodex) in GRUR 2011, 431, 432, Tz. 13.

1013 Urteil des BGH v. 7. 2. 2006 - KZR 33/04 (OLG Hamburg) (Probeabonnement), Tz. 19 in NJW 2006, 2627 mit weiteren Nennungen, Urteil des BGH v. 09.09.2010 - I ZR 157/08 (OLG München) (FSA-Kodex) in GRUR 2011, 431, 432, Tz. 13. 
Standpunkt der betroffenen Allgemeinheit als unlauter einzuordnen ist. ${ }^{1014}$ Es bestand somit eine echte Indizwirkung, da entsprechende Verhaltensweisen zunächst einmal als unlauter galten und diese Unlauterkeit lediglich überprüft wurde. Nach der heutigen Rechtsprechung kommt Verhaltenskodizes aber keine solche Indizwirkung mehr zu. Vielmehr kommt ihnen nur noch die Funktion zu, Hinweise auf die in einer Branche bestehende tatsächliche Übung zu liefern, welche sodann bei der Ermittlung der anständigen Gepflogenheiten Berücksichtigung finden können. Insofern erscheint selbst die Anerkennung einer allenfalls indizielle Bedeutung ${ }^{1015}$ missverständlich, auch wenn erkennbar ist, dass der BGH die Begrifflichkeit nur vereinzelt verwendet und insbesondere in seinem Einleitungssatz vollständig auf sie verzichtet. ${ }^{1016}$

\section{Bewertung der Rechtsprechung, insbesondere im Hinblick auf ihre Vereinbarkeit mit der UGP-Richtlinie}

Da die Rechtsprechung des UWG im Grundsatz noch aus der Zeit vor der Vollharmonisierung des europäischen Lauterkeitsrechts im b2c-Bereich herrührt, kommt der Überprüfung ihrer Vereinbarkeit mit der UGP-Richtlinie eine besondere Bedeutung zu. Ansatzpunkt für die Ermittlung der Bedeutung von Verstößen gegen Verhaltenskodizes für die Unlauterkeit ist Erwägungsgrund 20 der UGPRichtlinie, welcher die Hintergründe der Regelungen zu Verhaltenskodizes in der UGP-Richtlinie beleuchtet. Dessen Satz 2 stellt einen Bezug zwischen der beruflichen Sorgfalt als wesentliches Merkmal der Lauterkeit i. S. d. UGP-Richtlinie mit Verhaltenskodizes her. ${ }^{1017}$ Demnach ist es nach Ansicht der Richtliniengeber in „Branchen, in denen es sperifische zwingende Vorschriften gibt, die das Verhalten von Gewerbetreibenden regeln, $\{. .$.$\} zweckmäßig, dass aus diesen auch die Anforderungen an die berufliche$ Sorgfalt in dieser Branche ersichtlich sind."

\section{a. Beschränkung auf Branchenregelungen}

Satz 2 von Erwägungsgrund 20 der UGP-Richtlinie lässt sich somit entnehmen, dass im Hinblick auf die Bedeutung von Verhaltenskodizes zur Bestimmung der beruflichen Sorgfalt nur Verhaltenskodizes in Betracht kommen, die für eine gesamte Branche gelten. Hinsichtlich dieser Beschränkung auf Branchenregelungen

\footnotetext{
1014 Urteil des BGH 08.11.1990 - I ZR 48/89 (OLG Hamm) (Wettbewerbsrichtlinie der Privatwirtschaft) in GRUR 1991, 462, 463.

1015 Urteil des BGH v. 09.09.2010 - I ZR 157/08 (OLG München) (FSA-Kodex) in GRUR 2011, 431, 432, Tz. $13 \mathrm{f}$.

1016 In diesem spricht der BGH nicht von einer allenfalls indiziellen Bedeutung, sondern von einer nur begreñten Bedeutung, Urteil des BGH v. 09.09.2010 - I ZR 157/08 (OLG München) (FSAKodex) in GRUR 2011, 431, 432, Tz. 13.

1017 Zur Vergleichbarkeit der Begrifflichkeiten berufliche bzw. fachliche Sorgfalt und Lauterkeit bzw. anständige Gepflogenheiten vgl. Kapitel 6D.I.
} 
ist festzustellen, dass die Rechtsprechung des BGH ebenfalls nicht sämtliche Verhaltenskodexes i. S. d. $\int 5$ Abs. 1 Nr. 5 UWG betrifft, sondern lediglich „Regeln, die sich ein Verband oder ein sonstiger Zusammenschluss von Verkebrsbeteiligten gegeben bat".1018 Die Rechtsprechung des BGH stimmt damit insoweit mit der UGP-Richtlinie überein, als sie für die Ermittlung der anständigen Gepflogenheiten nur auf Verhaltenskodizes zurückgreift, denen aufgrund ihrer Urheber oder Beteiligten ein gewisses Gewicht für die Ermittlung der anständigen Gepflogenheiten in einer Branche beizumessen ist. Eine solche Beschränkung ist sinnvoll, da beispielsweise die von einem Unternehmer in einem nur für ihn geltenden Verhaltenskodex ${ }^{1019}$ selbst aufgestellten Angaben zu den anständigen Gepflogenheiten in seiner Branche diesbezüglich untauglich sind. ${ }^{1020}$ Gleiches gilt für einen Verhaltenskodex, der nicht die gesamte Branche repräsentiert. ${ }^{1021}$

Dem zweiten Satz von Erwägungsgrund 20 der UGP-Richtlinie können jedoch noch über die Einschränkungen der Rechtsprechung des BGH hinausgehende Anforderungen an Verhaltenskodizes entnommen werden. Denn dieser beschränkt sich nicht auf Branchenregelungen, sondern setzt bezüglich deren Inhalt voraus, dass diese sperifische zwingende Vorschriften über das Verhalten vorgeben. Dabei darf der Begriff der zwingenden Vorschrift nicht im Sinne eines gesetzlichen Zwangs verstanden werden, da dies im Widerspruch zum Wesensmerkmal der Freiwilligkeit eines Verhaltenskodexes steht. ${ }^{1022}$ Vielmehr ist dieser ausdrückliche Hinweis auf die Notwendigkeit zwingender spezifischer Vorschriften im Hinblick auf die Maßgaben aus Art. 6 Abs. 2 lit. b) UGP-Richtlinie so zu verstehen, dass der Verhaltenskodex bestimmte Verhaltensweisen verpflichtend vorgeben muss, so dass Handlungsempfehlungen und Absichtserklärungen wiederum ${ }^{1023}$ nicht als ausreichend anzuerkennen sind. ${ }^{1024}$

Auch die Beschränkung des BGH, nur solchen Verstößen gegen Verhaltenskodizes indizielle Bedeutung zuzugestehen, die von ihrem Unrechtsgehalt her den gesetzlichen Unlauterkeitstatbeständen entsprechen, steht im Einklang mit der UGP-Richtlinie. Denn auch wenn der Begriff des Verhaltenskodexes nach der UGP-Richtlinie im Hinblick auf den Gegenstand der Verpflichtung weit zu fassen ist ${ }^{1025}$, erfasst der Erwägungsgrund 20 der UGP-Richtlinie, welcher die Bedeutung von Verhaltenskodizes für die Bestimmung der beruflichen Sorgfalt betrifft, nur solche Verhaltenskodizes, „die es Gewerbetreibenden ermöglichen, die Grundsätze dieser

\footnotetext{
1018 Urteil des BGH v. 09.09.2010 - I ZR 157/08 (OLG München) (FSA-Kodex) in GRUR 2011, 431, 432, Tz. 13.

1019 Vgl. Kapitel 1C.I.

1020 Erdmann in: Gloy/Loschelder/Erdmann, [ 37, Rn. 8.

1021 Alexander, GRURInt 2012, 965, 972.

1022 Vgl. Kapitel 1A.

1023 Vgl. Kapitel 5B.III.1.

1024 Alexander, GRURInt 2012, 965, 972.

1025 Vgl. Kapitel 3C.
} 
Richtlinie in spezifischen Wirtschaftsbranchen wirksam anzuwenden." Die UGP-Richtlinie setzt somit für die Zulassung von Verhaltenskodizes zur Bestimmung der beruflichen Sorgfalt ebenfalls voraus, dass sie Verpflichtungen enthalten, die mit den Tatbeständen der UGP-Richtlinie und damit ihren Grundsätzen vergleichbar sind. Zudem ist der Begriff der beruflichen Sorgfalt als lauterkeitsrechtlicher Maßstab der Unlauterkeit inhaltlich durch die Schutzzwecke des Lauterkeitsrechts begrenzt. ${ }^{1026}$ Eine Verhaltensweise, die das Lauterkeitsrecht nicht betrifft, kann aus diesem Grund auch niemals eine Verletzung der beruflichen Sorgfalt darstellen und damit auch nicht aufgrund von Art. 5 Abs. 2 UGP-Richtlinie unlauter sein. 1027 Verstoßen entsprechende Verhaltensweisen gegen Verhaltenskodizes kommt eine lauterkeitsrechtliche Sanktionierung nur in Betracht, wenn die Voraussetzungen des Art. 6 Abs. 2 lit. b) UGP-Richtlinie vorliegen.

b. Kein Automatismus zwischen dem Verstoß einer geschäftlichen Handlung gegen einen Verhaltenskodex und deren Unlauterkeit

Der Rechtsprechung des BGH ist dahingehend zuzustimmen, dass in keinem Fall ein Automatismus anerkannt werden darf, nach dem Verhaltensweisen, welche gegen „Regeln, die sich ein Verband oder ein sonstiger Zusammenschluss von Verkehrsbeteiligten gegeben hat" verstoßen, als unlauter einzuordnen sind. ${ }^{1028}$ Denn da die Unlauterkeit einer geschäftlichen Handlung sich auf einen Verstoß gegen die anständigen Gepflogenheiten gründet ${ }^{1029}$, würde ein solcher Automatismus bedeuten, dass die anständigen Gepflogenheiten in einer bestimmten Branche durch solche Regelwerke festgesetzt würden. Dies ist jedoch aus mehreren Gründen abzulehnen. Zunächst einmal ist bezüglich der damit einhergehenden Rechtssetzung durch Private den verfassungsrechtlichen Bedenken des BGH zuzustimmen ${ }^{1030}$, da Verhaltenskodizes in aller Regel Verhaltensvorgaben enthalten, die über die gesetzlichen Verhaltensvorgaben hinausgehen. ${ }^{1031}$ Denn es ist Aufgabe des Gesetzgebers festzulegen, wo im Einzelfall die Grenze zwischen erlaubten und verbotenen Verhalten verläuft. ${ }^{1032}$ Zudem wäre ein solcher Automatismus auch im Hinblick auf das besondere Verhältnis von UWG und GWB1033 bedenklich. ${ }^{1034}$ Denn sobald aufgrund solcher Verhaltenskodizes geschäftliche Handlungen verboten würden,

\footnotetext{
1026 Alexander, GRURInt, 2012, 965, 972.

1027 Alexander, GRURInt, 2012, 965, 972.

1028 Urteil des BGH v. 09.09.2010 - I ZR 157/08 (OLG München) (FSA-Kodex) in GRUR 2011, 431, 432, Tz. 13.

1029 Vgl. Kapitel 6D.I.

1030 Urteil des BGH v. 09.09.2010 - I ZR 157/08 (OLG München) (FSA-Kodex) in GRUR 2011, 431, 432, Tz. 14.

1031 Die Ausnahme bilden die sogenannten deklaratorischen Erklärungen, vgl. Kapitel 3A.

1032 Sosnitza in: MünchKomm, Grundl., Rn. 55.

1033 Sosnitza in: FS für Bechtold, S. 517.

1034 Bornkamm in: Köhler/Bornkamm, UWG, \ 5, Rn. 5.165 f., vgl. Kapitel 3F.
} 
die nach dem UWG zulässig sind, wären diese Verhaltenskodizes als wettbewerbsbeschränkende Vereinbarungen anzusehen, welche nach \ 1 GWB verboten sind. 1035

Darüber hinaus widerspricht ein solcher Automatismus den Wertungen und Regelungszielen der UGP-Richtlinie. Dies zeigt sich bereits dadurch, dass schon die zur Vorbereitung des Grünbuchs Verbraucherschutz. der Europäischen Kommission von der Generaldirektion Gesundheit und Verbraucher beauftragte ${ }^{1036}$ und unter der Federführung von Micklitz erstellte Studie des Instituts für Europäisches Wirtschafts- und Verbraucherrecht e. V. eine solche Verlagerung der Bestimmung der Anforderungen an die berufliche Sorgfalt und die damit einhergehende Rechtssetzung durch Private ablehnte. Zwar schlug diese vor, sogenannte Marketingstandards ${ }^{1037}$ zur Ausfüllung der Generalklausel heranzuziehen. ${ }^{1038}$ Sie betont aber gleichfalls, „dass die endguittige Entscheidung dariber, was anständige Gepflogenheiten sind, nicht denjenigen wiberlassen werden dürfe, die sie herausbilden."1039 Zudem ergibt sich aus Art. 6 Abs. 2 lit. b) UGP-Richtlinie, dass die Richtliniengeber Verstöße gegen Verpflichtungen aus Verhaltenskodizes nur unter Vorliegen besonderer Voraussetzungen ${ }^{1040}$ als irreführend und damit unlauter ${ }^{1041}$ erachtet haben. Diese Wertung würde jedoch leerlaufen, wenn jeder Verstoß gegen einen Verhaltenskodex als Verstoß gegen die anständigen Gepflogenheiten und damit als unlauter anzusehen wäre. Zudem betrifft Art. 6 Abs. 2 lit. b) UGP-Richtlinie nur diejenigen, welche sich selbst auf den Verhaltenskodex verpflichtet haben und auf ihre Bindung an diesen hingewiesen haben. Von einer Festsetzung der anständigen Gepflogenheiten durch Verhaltenskodizes wäre jedoch der gesamte Geschäftsverkehr betroffen, so dass letztlich derjenige, welcher sich selbst überhaupt nicht durch einen Verhaltenskodex auf ein bestimmtes Verhalten verpflichtet hat, weitergehenden Verpflichtungen unterliegen würde, als die UGP-Richtlinie in Art. 6 Abs. 2 lit. b) für denjenigen vorsieht, der sich selbst freiwillig auf einen Verhaltenskodex verpflichtet hat. Eine solche Verpflichtung Unbeteiligter durch Verhaltenskodizes widerspricht zudem dem Grundsatz der Freiwilligkeit der Verpflichtung durch einen Verhaltenskodex. ${ }^{1042}$ Vor diesem Hintergrund hat die Europäi-

\footnotetext{
1035 Bornkamm in: Köhler/Bornkamm, UWG, \5, Rn. 5.165.

1036 Glöckner in: Harte-Bavendamm/Henning-Bodewig, UWG, Einl. B., Rn. 221.

1037 Diese Marketingstandards sind jedoch eher mit den Regelungen im Vereinigten Königreich vergleichbar (vgl. Kapitel 2B.I.2.b.) und stellen damit nur einen Teilbereich der vom Begriff des Verhaltenskodexes i. S. d. UWG-Richtlinie umfassten Regelungen dar (vgl. Kapitel 3.), so dass der Studie diesbezüglich ohnehin nur begrenzte Aussagekraft zugestanden werden kann.

1038 Micklitz-Studie, Band I, S. 59.

1039 Zusammenfassung der Micklitz-Studie, S. 6, abrufbar unter http://ec.europa.eu/ consumers/rights/studies_reports_en.print.htm\#studies, zuletzt abgerufen am 28.03.2013.

1040 Vgl. zu den Voraussetzungen Kapitel 5B.

1041 Art. 4 lit. a) UGP-Richtlinie.

1042 Vgl. Kapitel 1A
} 
sche Kommission bereits in ihrer Mitteilung zu den Folgemaßnahmen zum Grünbuch über Verbraucherschutz, in der EU und damit schon vor Erstellung des ersten Vorschlages für die UGP-Richtlinie feststellt, dass Verhaltenskodizes keine Anwendung auf Wirtschaftsakteure finden sollen, „die derartigen Selbstregelungen nicht beigetreten sind.“"1043

c. Ermittlungshilfe zur Bestimmung der fachlichen Sorgfalt / Indizwirkung von Verstößen gegen Verhaltenskodizes

Die vom BGH als allenfalls beziehungsweise lediglich indiriell bezeichnete Bedeutung von Verstößen gegen Verhaltenskodizes für die Ermittlung der Unlauterkeit von geschäftlichen Handlungen aufgrund der sich eventuell aus den Verhaltenskodizes ergebenden tatsächlichen Übung beziehungsweise der anständigen Gepflogenheiten in einer Branche ${ }^{1044}$ entspricht der Bedeutung von Verhaltenskodizes für die Bestimmung der beruflichen Sorgfalt ${ }^{1045}$ aus der UGP-Richtlinie. Deren Verständnis der dahingehenden Bedeutung von Verhaltenskodizes ergibt sich ebenfalls aus Satz 2 von Erwägungsgrund 20 der UGP-Richtlinie. Nach diesem erachten es die Richtliniengeber als zweckmäßig, dass aus Verhaltenskodizes „anch die Anforderungen an die berufliche Sorgfalt in dieser Branche ersichtlich sind." Hieraus lässt sich entnehmen, dass die Richtliniengeber Verhaltenskodizes mitnichten zugestehen, die berufliche Sorgfalt zu definieren. Sie erkennen diese vielmehr lediglich als eine von vielen Quellen an, die Erkenntnisse darüber liefern, welches Verständnis die jeweilige Branche von der für sie bestehenden berufliche Sorgfalt hat. ${ }^{1046} \mathrm{Um}$ diese als Erkenntnisquelle nutzen zu können, fordert die UGP-Richtlinie die Urheber entsprechender Verhaltenskodizes auf, ihr Verständnis der beruflichen Sorgfalt in ihrer Branche zu kodifizieren. ${ }^{1047}$ Etwas weitergehender war diesbezüglich noch die Formulierung in dem Richtlinienvorschlag der Generaldirektion Gesundheit und Verbraucher. ${ }^{1048}$ In dessen Erwägungsgrund 14, aus welchem der Erwägungsgrund 20 der UGP-Richtlinie hervorgegangen ist, hieß es, Verhaltenskodizes ,können den nationalen Bebörden die Feststellung erleichtern, welche Anforderungen in der konkreten Branche an die berufliche Sorgfalt ₹u stellen sind." ${ }^{1049}$ Auch wenn es wohl zu weit geht, aus dieser Formulierung eine Indizwirkung abzuleiten, so ist ihr doch zumindest die Empfehlung zu entnehmen, zur Ermittlung der beruflichen Sorgfalt auf Verhaltenskodizes zurückzugreifen. Eine Auslegung der UGP-Richtlinie unter

\footnotetext{
1043 KOM (2002) 289 endgültig, S. 13, Tz. 29.

1044 Vgl. Kapitel 6D.II.1.

1045 Für die Definition des Begriffs vgl. Fn. 982.

1046 Erdmann in: Gloy/Loschelder/Erdmann, \$ 37, Rn. 8.

1047 A. A. Heidinger in: Wiebe/Kodek, UWG, $\int 1$, Rn. 43, der dieser Formulierung entnimmt, dass lediglich angeregt wird, dass sich die Urheber von Verhaltenskodizes an der UGP-Richtlinie orientieren.

1048 Vgl. Kapitel 2B.I.1.a.

1049 KOM (2003) 356 endgültig, Erwägungsgrund 14.
} 
Rückgriff auf diese Formulierung kommt jedoch nicht in Betracht. Denn die fehlende Übernahme spricht gerade dafür, dass die Richtliniengeber sich dem Vorschlag nicht angeschlossen haben. Dies gilt neben der grundsätzlich geringen Bedeutung von Gesetzgebungsmaterialien für die Auslegung des Gemeinschaftsrechts vorliegend im Besonderen für die Auslegung der UGP-Richtlinie im Hinblick auf den Komplex der Selbstregulierung, da sich das von der Generaldirektion Gesundheit und Verbraucher vorgeschlagene Regelungskonzept nicht durchgesetzt hat. ${ }^{1050}$ So beruht die Auslegungsmaßgabe aus Erwägungsgrund 14 des Vorschlages für die UGP-Richtlinie auf der Annahme, dass ,es ein Potenzial für EUweit anwendbare Kodizes zur Förderung der Konvergenz. binsichtlich der an die berufliche Sorgfalt zu stellenden Erwartungen" gibt. ${ }^{1051}$ Diese Verhaltenskodizes auf Gemeinschaftsebene, welche der Vorschlag für die UGP-Richtlinie noch als eigene Kategorie von Verhaltenskodizes mit eigener Definition in Art. 2 lit. h) ${ }^{1052}$ enthielt, wurden jedoch überhaupt nicht in die UGP-Richtlinie übernommen.

Die Bedeutung von Verhaltenskodizes zur Bestimmung der beruflichen Sorgfalt beschränkt sich damit auf die Funktion als Erkenntnisquelle über das Verständnis der jeweiligen Branche über die Anforderungen an die berufliche Sorgfalt. Dementsprechend geht es bereits zu weit, wenn Verhaltenskodizes zugestanden wird, die Sorgfaltsanforderungen in Bezug auf bestimmte Wirtschaftszweige oder einzelne geschäftliche Handlungen konkretisieren und präzisieren zu können. ${ }^{1053}$ Denn die von einem Unternehmen anzuwendende berufliche Sorgfalt kann nicht individuell durch die von dem Unternehmen im Rahmen eines Verhaltenskodexes abgegebenen Verpflichtungen auf ein bestimmtes Verhalten für dieses Unternehmen konkretisiert werden, da sie für alle Unternehmen einer Branche gleich ist. ${ }^{1054}$ In keinem Fall vereinbar mit der UGP-Richtlinie wäre daher eine tatsächliche Indizwirkung entsprechend der mittlerweile vom BGH geänderten Rechtsprechung aus seiner Entscheidung Werberichtlinien der Privatwirtschaft. ${ }^{1055}$

\footnotetext{
1050 Micklitz in: MünchKomm zum Lauterkeitsrecht, EG, E, Rn. 208, vgl. Kapitel 2B.I.2.a.

1051 KOM (2003) 356 endoültig, Begründung Nr. 73.

1052 Art. 2 lit. h) des Vorschlags für die UGP-Richtlinie (KOM (2003) 356 endgültig): ,,, Verbaltenskodex auf Gemeinschaftsebene" ein Verbaltenskodex, der es jedem Gewerbetreibenden aus jedem Mitgliedstaat, der die Anforderungen des Kodex erfüllt, erlaubt, sich obne jede Diskriminierung an diesem Kodex zu beteiligen, und der geeignete und wirksame Verfabren für die Überwachung und Durchsetzung seiner Bestimmungen entbält;“"

1053 Heidinger in: Wiebe/Kodek, UWG, \1, Rn. 43, der Verhaltenskodizes nicht mehr als die Funktion als Orientierungshilfe bei der Ausfüllung der Unlauterkeit bzw. der fachlichen/beruflichen Sorgfalt zugesteht, a. A. Alexander, GRURInt 2012, 965, 972, Fezer in: Fezer, UWG, $₫ 2$ Nr. 5 , Rn. 36.

1054 A. A. Fezer in: Fezer, UWG, $\$ 2$ Nr. 5, Rn. 36.

1055 Alexander, GRURInt 2012, 965, 972.
} 


\section{Zusammenfassung}

Geschäftliche Handlungen, die gegen einen Verhaltenskodex verstoßen, sind wie jede andere geschäftliche Handlung nur dann nach der Generalklausel des \ 3 Abs. 1 UWG unlauter, wenn der Verstoß gegen den Verhaltenskodex den anständigen Gepflogenheiten widerspricht. Wird eine solche geschäftliche Handlung gegenüber einem Verbraucher vorgenommen, setzt eine Unzulässigkeit nach der Verbrauchergeneralklausel des $₫ 3$ Abs. 2 Satz 1 UWG zunächst die Nichteinhaltung der fachlichen Sorgfalt voraus. Bei der Ermittlung der Frage, ob eine geschäftliche Handlung gegen die anständigen Gepflogenheiten beziehungsweise die fachliche Sorgfalt verstößt, kommt der Tatsache, dass die geschäftliche Handlung gegen einen Verhaltenskodex verstößt, keine direkte Bedeutung zu. ${ }^{1056}$ Vielmehr beschränkt sich die Bedeutung von Verhaltenskodizes in diesem Zusammenhang darauf, dass diese als Erkenntnisquelle herangezogen werden (können), wenn es darum geht, die anständigen Gepflogenheiten beziehungsweise die fachliche Sorgfalt zu bestimmen. ${ }^{1057}$ Voraussetzung hierfür ist jedoch, dass es sich um einen Verhaltenskodex handelt, der für eine gesamte Branche bestimmte Verhaltensweisen verpflichtend vorschreibt. ${ }^{1058}$ Andere Verhaltenskodizes wie beispielsweise unternehmenseigene Verhaltenskodizes können dahingegen nicht herangezogen werden.

Eine geschäftliche Handlung, die gegen einen Verhaltenskodex verstößt, ist somit niemals aufgrund des Verstoßes gegen den Verhaltenskodex aufgrund der Generalklauseln unlauter. Dies ist nur unter der Voraussetzung der Fall, dass die durch den Verhaltenskodex vorgeschriebene Verhaltensweise zufälligerweise exakt der fachlichen Sorgfalt beziehungsweise den anständigen Gepflogenheiten entspricht und ein Verstoß gegen den Verhaltenskodex damit einen Verstoß gegen diese darstellt. In Betracht kommen hierfür zudem nur solche Verstöße gegen Verhaltenskodizes, die von ihrem Unrechtsgehalt her den Unlauterkeitstatbeständen des UWG entsprechen. ${ }^{1059}$ Denn die Begriffe der fachlichen Sorgfalt sowie der anständigen Gepflogenheiten sind inhaltlich durch die Schutzzwecke des UWG begrenzt. 1060 Ausgeschlossen sind damit Verstöße gegen Verhaltenskodizes wie den in den „Leitlinien zur Umsetzung/Anwendung der Richtlinie 2005/29/EG über unlautere Geschäftspraktiken" als Beispiel angeführten Verhaltenskodex über nach-

\footnotetext{
1056 Urteil des BGH v. 09.09.2010 - I ZR 157/08 (OLG München) (FSA-Kodex) in GRUR 2011, 431, 432, Tz. 13.

1057 Erwägungsgrund 20 der UGP-Richtlinie, Urteil des BGH v. 09.09.2010 - I ZR 157/08 (OLG München) (FSA-Kodex) in GRUR 2011, 431, 432, Tz. 13 f.

1058 Erwägungsgrund 20 der UGP-Richtlinie.

1059 Urteil des BGH v. 09.09.2010 - I ZR 157/08 (OLG München) (FSA-Kodex) in GRUR 2011, 431, 432, Tz. 14.

1060 Urteil des BGH v. 09.09.2010 - I ZR 157/08 (OLG München) (FSA-Kodex) in GRUR 2011, 431, 432, Tz. 14, Alexander, GRURInt 2012, 965, 972.
} 
haltige Holznutzung. ${ }^{1061}$ Denn die Verwendung von Holz aus Rodungsgebieten entspricht in ihrem Unrechtsgehalt keinem der Unlauterkeitstatbestände des UWG. ${ }^{1062}$ Gleiches dürfte für eine Vielzahl der Verpflichtungen in Verhaltenskodizes aus den Bereichen Ökologie, Ethik und soziale Mindeststandards gelten.

Dies entspricht auch der Rechtsprechung des BGH im Hinblick auf die Bedeutung von Verstößen gegen Verhaltenskodizes für die Unlauterkeit aufgrund der Generalklausel des $\ 3$ Abs. 1 UWG. Dabei ist unerheblich, dass der BGH weiterhin die Formulierung der indiziellen Bedeutung verwendet. ${ }^{1063}$ Denn tatsächlich hat der BGH diese Indizrechtsprechung, welche den Maßgaben der UGPRichtlinie widersprechen würde, bereits mit seiner Probeabonnemententscheidung ${ }^{1064}$ aufgegeben. Seither nutzt der BGH Verhaltenskodizes in Übereinstimmung mit den Maßgaben der UGP-Richtlinie im Rahmen der Generalklausel nur noch zur Ermittlung möglicherweise bestehender tatsächlicher Übungen, die jedoch, selbst wenn sie bestehen, nicht zwangsläufig die anständigen Gepflogenheiten definieren. ${ }^{1065}$

${ }^{1061}$ Leitlinien zur Umsetzung der UGP-Richtlinie, SEK(2009)1666, Punkt 2.5.6., S. 51 f., vgl. für das Beispiel Kapitel 3C.

1062 Unabhängig davon ist natürlich die Angabe, ein Möbel sei aus entsprechendem Holz gefertigt, irreführend, wenn dies tatsächlich nicht der Fall ist.

1063 Urteil des BGH v. 09.09.2010 - I ZR 157/08 (OLG München) (FSA-Kodex) in GRUR 2011, 431, 432, Tz. $13 \mathrm{f}$.

1064 Urteil des BGH v. 7. 2. 2006 - KZR 33/04 (OLG Hamburg) (Probeabonnement) in NJW 2006, $2627 \mathrm{ff}$.

1065 Urteil des BGH v. 09.09.2010 - I ZR 157/08 (OLG München) (FSA-Kodex) in GRUR 2011, $431 \mathrm{ff}$. 


\section{Kapitel 7}

\section{Schlussbetrachtung}

Die im Rahmen der Umsetzung der UGP-Richtlinie in das UWG von Dreyer mit Skepsis aufgeworfene Frage, ob das Lauterkeitsrecht von jetzt an für die Einhaltung von Selbstverpflichtungen zuständig sei und damit zum Motor für deren Durchsetzung werde ${ }^{1066}$, muss mit Jein beantwortet werden. Ja insoweit, als sich mit $\ 5$ Abs. 1 Satz 2 Nr. 6 UWG nunmehr eine Regelung im UWG befindet, die den Verstoß gegen einen Verhaltenskodex unter bestimmten Voraussetzungen als irreführende geschäftliche Handlung und damit als unlauter einordnet. Nein insoweit, als sich an der grundsätzlichen Bedeutung von Verhaltenskodizes für die Beurteilung der Unlauterkeit beziehungsweise der Unzulässigkeit von geschäftlichen Handlungen aufgrund der Generalklauseln des $₫ 3$ UWG auch unter dem UWG 2008 nichts geändert hat.

Bezüglich des auf Verhaltenskodizes i. S. d $\ 2$ Abs. 1 Nr. 5 UWG beschränkten Anwendungsbereiches der mit dem „Ersten Gesetz, zur Änderung des Gesetzes gegen den unlauteren Wettbewerb" in das UWG eingeführten Spezialregelungen zu Verhal-

1066 Dreyer, WRP 2007, 1294, 1295 die bereits in der Unterzeile der Überschrift ihres Artikels die Frage stellt, ob dass Wettbewerbsrecht mit dem „Ersten Gesetz zur Änderung des Gesetzes gegen den unlauteren Wettbewerb“ „zum Motor für die Durchsetzung vertraglicher Verpflicbtungen wird“. 
tenskodizes ${ }^{1067}$ ist festzustellen, dass der Wortlaut der Definition eines Verhaltenskodexes in $\ 2$ Abs. 1 Nr. 5 UWG zu weit gefasst ist. ${ }^{1068}$ Denn unter diesen können nahezu sämtliche Verträge gefasst werden, durch welche sich ein Unternehmer verpflichtet. Würde man somit allein auf den Wortlaut abstellen, wären auch Regelwerke wie beispielsweise Vertriebsbindungssysteme als Verhaltenskodizes i. S. d. UWG anzusehen, die nichts mit den Verhaltenskodizes zu tun haben, an welchen sich die Richtliniengeber orientiert haben. ${ }^{1069}$ Verhaltenskodizes i. S. d. UWG können daher unter Einschränkung des weiten Wortlauts von $₫ 2$ Abs. 1 Nr. 5 UWG aufgrund des Willens der Richtliniengeber in Orientierung am allgemeinen Sprachgebrauch nur Regelwerke sein, die über die jeweils geltenden gesetzlichen Anforderungen hinaus die Verpflichtung zur Einhaltung gesellschaftliche Werte einzuhalten. Insofern scheiden gewöhnliche Verträge mit wirtschaftlichem Hintergrund und wirtschaftlich äquivalenten Ansprüchen der Parteien aus. Ein lauterkeitsrechtlicher Bezug der Verpflichtung ist dahingegen nicht erforderlich, so dass auch Verpflichtungen aus dem ökologischen, ethischen oder sozialen Bereich ausreichen. ${ }^{1070}$

Hinsichtlich des Grades der Verpflichtung bestehen dahingegen keine Vorgaben, so dass auch Absichtserklärungen ${ }^{1071}$ Verhaltenskodizes i.S. d. \2 Abs. 1 Nr. 5 UWG sind. Allerdings sind entsprechende Absichtserklärungen im Rahmen des UWG insoweit Verhaltenskodizes zweiter Klasse, als ein Verstoß gegen sie nicht zur Irreführung nach $\ 5$ Abs. 1 Satz 2 Nr. 6 UWG führen kann. Denn dieser erfasst nur Verstöße gegen Verhaltenskodizes, auf die sich ein Unternehmer ,verbindlich verpflichtet hat", was im Rahmen der richtlinienkonformen Auslegung der Norm den Ausschluss von Absichtserklärungen bedeutet. ${ }^{1072}$ Fehlt es an einem Verstoß gegen einen solchen Verhaltenskodex, kommt eine Irreführung nach $\ 5$ Abs. 1 Satz 2 Nr. 6 UWG entgegen dessen Wortlaut und dem Willen des deutschen Gesetzgebers 1073 nicht in Betracht, auch wenn trotzdem eine Täuschungseignung gegeben ist. Denn die Norm ist entsprechend der Vorgaben der UGP-Richtlinie auszulegen, welche den Verstoß gegen einen Verhaltenskodex zwingend voraussetzt. Aus diesem Grund wäre es sinnvoll, die Eingliederung der Ursprungsnorm aus Art. 6 Abs. 2 lit. b) UGP-Richtlinie in die Auflistung der irre-

\footnotetext{
1067 Nr. 1 und 3 des Anhangs zu \3 Abs. 3 UWG und \5 Abs. 1 Satz 2 Nr. 6 UWG, vgl. Kapitel 5.

1068 Hoeren, WRP 2009, 789, 793, Dreyer, WRP 2007, 1294, 1295 ff., Schmidbauer, WRP 2010, 593, 597.

1069 Schmidbauer, WRP 2010, S. 593, 597, Lamberti/Wendel, WRP 2009, 1479, 1481, wobei Letztere davon unabhängig für eine wortlautgetreue Auslegung plädieren.

1070 A. A. Dreyer, WRP 2007, 1294,1296, Büllesbach, S. 43.

1071 Zum Begriff der Absichtserklärung vgl. Kapitel 3D.

1072 Weitere Voraussetzung ist, dass der Unternehmer zuvor auf seine Bindung an den Verhaltenskodex hingewiesen hat. Diese Tatbestandsvoraussetzung dürfte jedoch nur in absoluten Ausnahmefällen nicht gegeben sein.

1073 Regierungsentwurf und Begründung zum UWG vom 20.08.2008, BT.-Drs. 16/10145, S. 24.
} 
führenden Umstände des $\int 5$ Abs. 1 Satz 2 UWG rückgängig zu machen und diesen stattdessen — wie im UWG Österreich erfolgt ${ }^{1074}$ — gemeinsam mit Art. 6 Abs. 2 lit. a) UGP-Richtlinie in $\int 5$ Abs. 2 UWG aufzunehmen. Dies wäre nicht nur aus Gründen der Rechtsklarheit und Rechtsvergleichbarkeit, sondern auch aus systematischen Gründen vorteilhaft. Denn Art. 6 Abs. 2 UGP-Richtlinie regelt „zwei besondere Fallgruppen irrefübrender geschäftlicher Handlungen"1075 mit immanentem Unrechtsgehalt. Dahingegen betreffen die lit. a) - g) von Art. 6 Abs. 1 UGPRichtlinie, welche den Umständen aus $\int 5$ Abs. 1 Satz 2 UWG entsprechen, wertneutrale Angaben in Geschäftspraktiken, die grundsätzlich zulässig und teilweise sogar verpflichtend sind. ${ }^{1076}$ Entsprechend bedarf es nur bei Letzteren der Festsetzung von Voraussetzungen, unter welchen diese Angaben irreführend sind. ${ }^{1077}$ Die von Art. 6 Abs. 2 UGP-Richtlinie erfassten Geschäftspraktiken besitzen jedoch bereits aus sich selbst heraus einen Unwertgehalt, so dass es für ihr Verbot keiner weiteren Voraussetzungen außer der Überschreitung der Relevanzschwelle bedarf, wie es im Einleitungssatz des Art. 6 Abs. 2 UGP-Richtlinie beziehungsweise in $\int 3$ Abs. 1 UWG bestimmt ist.

Nicht anwendbar ist $\int 5$ Abs. 1 Satz 2 Nr. 6 UWG auch dann, wenn ein Unternehmer gegen einen Verhaltenskodex verstößt, auf den er sich nicht verpflichtet hat. Aus diesem Grund muss sich die Unlauterkeit beziehungsweise Unzulässigkeit von solchen Verstößen gegen fremde Verhaltenskodizes unmittelbar aufgrund der Generalklauseln aus \3 Abs. 1 und Abs. 2 Satz 1 UWG ergeben. ${ }^{1078}$ Der hierfür notwendige Verstoß gegen die anständigen Gepflogenheiten ( 3 Abs. 1 UWG) beziehungsweise die fachliche Sorgfalt (\$ 3 Abs. 2 Satz 1 UWG) folgt jedoch weder unmittelbar aus dem Verstoß gegen einen Verhaltenskodex, noch ist er durch einen solchen indiziert. Vielmehr beschränkt sich die Bedeutung von Verhaltenskodizes in diesem Zusammenhang darauf, dass diese als Erkenntnisquelle herangezogen werden (können), wenn es darum geht, die anständigen Gepflogenheiten beziehungsweise die fachliche Sorgfalt zu bestimmen. Dies jedoch zudem nur unter der Voraussetzung, dass es sich um einen Verhaltenskodex handelt, welcher für eine gesamte Branche bestimmte Verhaltensweisen verpflichtend vorschreibt. Andere Verhaltenskodizes, wie beispielsweise unternehmenseigene Verhaltenskodizes, können dahingegen noch nicht einmal als eine solche Erkenntnisquelle herangezogen werden. Dies entspricht inhaltlich der Rechtspre-

1074 \ 2 Abs. 3 Nr. 2 ÖUWG.

1075 So wörtlich der deutsche Gesetzgeber, Begründung der Bundesregierung für den Gesetzentwurf zur Änderung des UWG vom 20.08.2008, BT.-Drs. 16/10145, S. 16.

1076 Wie beispielsweise zu den wesentlichen Merkmalen eines Produkts, Art. 6 Abs. 1 lit. b) UGPRichtlinie und Art. 7 Abs. 4 lit. a) UGP-Richtlinie, wobei die wesentlichen Merkmale nach den beiden Vorschriften nicht deckungsgleich sind, vgl. hierzu Kapitel 6C.II.1.a.bb.

1077 Unwahrheit oder sonstige Eignung zur Täuschung, Art. 6 Abs. 1 UGP-Richtlinie.

1078 Eine Unlauterkeit aufgrund eines Rechtsbruchs nach \4 Nr. 11 UWG kommt nicht in Betracht, da Verhaltenskodizes keine rechtlichen Vorschriften in dessen Sinne sind, vgl. Kapitel 6A.II. 
chung des BGH im Bezug auf die Bedeutung von Verstößen gegen Verhaltenskodizes für die Unlauterkeit aufgrund der Generalklausel des $₫ 3$ Abs. 1 UWG, auch wenn der BGH weiterhin die Formulierung der indiziellen Bedeutung verwendet. Tatsächlich hat der BGH diese Indizrechtsprechung, welche den Maßgaben der UGP-Richtlinie widersprechen würde, bereits mit seiner Probeabonnemententscheidung ${ }^{1079}$ aufgegeben und nutzt Verhaltenskodizes in Übereinstimmung mit den Maßgaben der UGP-Richtlinie seither im Rahmen der Generalklausel nur noch zur Ermittlung möglicherweise bestehender tatsächlicher Übungen. Diese tatsächlichen Übungen definieren jedoch, selbst wenn sie bestehen, nicht zwangsläufig die anständigen Gepflogenheiten. ${ }^{1080}$

Eine geschäftliche Handlung, die gegen einen fremden Verhaltenskodex verstößt, ist somit niemals aufgrund des Verstoßes gegen den Verhaltenskodex unlauter, sondern immer nur dann, wenn ein Verstoß gegen fachliche Sorgfalt beziehungsweise die anständigen Gepflogenheiten vorliegt.

Damit kommen aber bereits nur solche Verstöße gegen Verhaltenskodizes in Betracht, deren Unrechtsgehalt den Unlauterkeitstatbeständen des UWG entsprechen. Denn die Begriffe der fachlichen Sorgfalt sowie der anständigen Gepflogenheiten sind inhaltlich durch die Schutzzwecke des UWG begrenzt. Ausgeschlossen sind damit Verstöße gegen Verhaltenskodizes wie den in den „Leitlinien zur Umsetzung/Anwendung der Richtlinie 2005/29/EG über unlautere Geschäftspraktiken" als Beispiel für eine Irreführung nach Art. 6 Abs. 2 lit. b) UGP-Richtlinie angeführten Verhaltenskodex über nachhaltige Holznutzung. ${ }^{1081}$ Denn die Verwendung von Holz aus Rodungsgebieten entspricht in ihrem Unrechtsgehalt keinem der Unlauterkeitstatbestände des UWG. ${ }^{1082}$ Gleiches dürfte für die Mehrheit der Verpflichtungen in Verhaltenskodizes aus den Bereichen Ökologie, Ethik und soziale Mindeststandards gelten.

Im Gegensatz zu \5 Abs. 1 Satz 2 Nr. 6 UWG, der eine neue irreführende geschäftliche Handlung geschaffen hat, beschränkt sich die Auswirkung der als weitere Spezialregelungen zu geschäftlichen Handlungen im Zusammenhang mit Verhaltenskodizes in das UWG 2008 aufgenommenen Nr. 1 und Nr. 3 des Anhangs zu $₫ 3$ Abs. 3 UWG auf die besondere Rechtsfolge der unmittelbaren Unzulässigkeit der jeweiligen geschäftlichen Handlung nach \3 Abs. 3 UWG. Denn die unwahre Angabe, zu den Unterzeichnern eines Verhaltenskodexes zu gehören, ist nach der allgemeinen Regelung des $₫ 5$ Abs. 1 Satz 2 UWG ebenso irreführend

1079 Urteil des BGH v. 7. 2. 2006 - I .ZR 33/04 (OLG Hamburg) (Probeabonnement) in NJW 2006, $2627 \mathrm{ff}$.

1080 Urteil des BGH v. 09.09.2010 - I ZR 157/08 (OLG München) (FSA-Kodex) in GRUR 2011, 431 ff., vgl. Kapitel 6D.II.1.

${ }^{1081}$ Leitlinien zur Umsetzung der UGP-Richtlinie, SEK(2009)1666, Punkt 2.5.6., S. 51 f., vgl. für das Beispiel Kapitel 3C.

1082 Unabhängig davon ist natürlich die Angabe, ein Möbel sei aus entsprechendem Holz gefertigt, irreführend, wenn dies tatsächlich nicht der Fall ist. 
und damit unlauter wie die unwahre Angabe, ein Verhaltenskodex sei von einer öffentlichen oder einer anderen Stelle gebilligt worden. ${ }^{1083}$ Der Unterschied liegt somit allein darin, dass die aufgrund dieser unwahren Angaben nach den allgemeinen Regelungen des UWG irreführenden und damit unlauteren geschäftlichen Handlungen nach \3 Abs. 1 UWG zusätzlich noch geeignet sein müssen, die Interessen der Mitbewerber, Verbraucher oder sonstigen Marktteilnehmer spürbar zu beeinträchtigen, um als unzulässig eingestuft zu werden. An einer solchen Eignung fehlt es jedoch bei unwahren Angaben nur in absoluten Ausnahmefällen.

Aufgrund der allgemeinen Regelung des \5 Abs. 1 Satz 2 UWG können die Angaben aus den Nr. 1 und Nr. 3 des Anhangs zu $\int 3$ Abs. 3 UWG zudem nicht nur irreführend sein, wenn sie unwahr sind, sondern auch dann, wenn sie zwar objektiv richtig, aber trotzdem zur Täuschung geeignet sind. Im Hinblick auf die Angabe, zu den Unterzeichnern eines Verhaltenskodexes zu gehören, ist dies der Fall, wenn die Unterzeichnung oder der Verhaltenskodex unwirksam ist, so dass tatsächlich keine Bindung an diesen besteht. ${ }^{1084}$ Bei der objektiv korrekten Angabe, ein Verhaltenskodex sei von einer öffentlichen oder einer anderen Stelle gebilligt, liegt eine Täuschungseignung vor, wenn die Voraussetzungen der Billigung tatsächlich nicht vorliegen ${ }^{1085}$, die billigende Stelle irreführend bezeichnet ist ${ }^{1086}$ oder es ihr an der vom Empfänger der Angabe vorausgesetzten Neutralität fehlt. ${ }^{1087}$ Neben den Angaben aus Nr. 1 und Nr. 3 des Anhangs zu \ 3 Abs. 3 UWG kommen als weitere praxisrelevante Angaben im Zusammenhang mit Verhaltenskodizes Angaben über den Inhalt eines Verhaltenskodexes und somit die Verpflichtungen des jeweiligen Unternehmens sowie solche über die Überprüfung der Einhaltung in Betracht. Diese Angaben sind ebenfalls irreführend und damit unlauter, wenn sie unwahr oder auf sonstige Art und Weise zur Täuschung geeignet sind. ${ }^{1088}$

Im Hinblick auf die Irreführung durch Unterlassen im b2b-Bereich hat die Einführung des \5a UWG keine Veränderungen gebracht. Für geschäftliche Handlungen im b2c-Bereich bestehen besondere Informationspflichten lediglich im Rahmen von im Fernabsatz oder außerhalb geschlossener Geschäftsräume geschlossenen Verträgen. Denn für diese sieht Art. 6 Abs. 1 lit. n.) Verbraucherrechte-Richtlinie eine Informationspflicht über bestehende Verhaltenskodizes und über die Möglichkeit des Bezugs von Exemplaren dieses Verhaltenskodexes vor, so dass diese Information gemäß \5a Abs. 4 UWG für Verbraucher unwiderlegbar als wesentlich gilt. $\mathrm{Da}$ diese Informationspflicht in Art. 246a \$1

\footnotetext{
1083 Vgl. Kapitel 6B.I.

1084 Vgl. Kapitel 6B.II.2.

1085 Vgl. Kapitel 6B.II.1.a.

1086 Vgl. Kapitel 6B.II.1.c.

1087 Vgl. Kapitel 6B.II.1.b.

1088 Vgl. Kapitel 6B.III.
} 
Nr. 10 EGBGB übernommen wurde, stellt die fehlende Information gleichzeitig die Nichteinhaltung einer gesetzlichen Vorschrift dar. Entsprechend sind diese geschäftlichen Handlungen unlauter gemäß $\ 4$ Nr. 11 UWG.

Abseits von im Fernabsatz oder außerhalb geschlossener Geschäftsräume geschlossener Verträge stellt das Bestehen und Nichtbestehen eines Verhaltenskodexes beziehungsweise die Einhaltung eines Verhaltenskodexes jedoch im b2cBereich keine wesentliche Information i. S. d. \5a UWG dar. 


\section{Literaturverzeichnis}

Alexander, Christian: Verhaltenskodizes im europäischen und deutschen Lauterkeitsrecht, GRURInt 2012, S. 965-973.

- Die Umsetzung der Verbraucherrechte-Richtlinie und die Auswirkungen auf das Lauterkeitsrecht, WRP 2014, S. 501-509.

Arndt, Domenik E.: Sinn und Unsinn von Soft Law — Prolegomena zur Zukunft eines indeterminierten Paradigmas, Baden-Baden, 2011.

Beater, Axel: Unlauterer Wettbewerb, Tübingen, 2011.

Bechtold, Rainer, Bosch Wolfgang: Kartellgesetz, Gesetz gegen Wettbewerbsbeschränkungen (\$S 1-96, 130, 131), Kommentar, 7. Auflage 2013.

Belanger, Jean, Topalovic Peter, Krantžberg Gail: Responsible Care: History \& Development, 2009.

Berlit, Wolfgang: Wettbewerbsrecht, 8. Auflage, München, 2011.

- Der öffentlich-rechtliche Vertrag, informales Handeln der Behörden und Selbstverpflichtungen Privater als Instrumente des Umweltschutz, Köln, 1986.

Biedermann, Reinhard: Sozialstandards durch Private Governance - Zwei-StufenKooperation in der globalen Spielzeugbranche, Baden-Baden, 2007.

Birk, Axel: Corporate Responsibility, unternehmerische Selbstverpflichtungen und unlauterer Wettbewerb, GRUR 2011, S. 196-203.

Blasek, Katrin: Anmerkungen zum Urteil des LG Darmstadt v. 22.11.2008 - 22 O 100/08 („,BVDVA-geprüft“) in MMR 2009, S. 279- 280.

Büllesbach, Eikee: Auslegung der irreführenden Geschäftspraktiken des Anhangs I der Richtlinie 2005/29/EG über unlautere Geschäftspraktiken, München, 2008.

Bornkamm, Joachim: Das Verhältnis von Kartellrecht und Lauterkeitsrecht: Zwei Seiten derselben Medaille? in: Festschrift für Irmgard Griss, herausgegeben von Brigitte Schenk, Elisabeth Lovrek, Gottfried Musger und Matthias Neumayer, 2011, S. 79- 93.

Daniel, Hans F.: Die Entwicklung einer Initiative — Gestartet als Idee — angekommen als Erfolg, Chemie Report Special, 09.2011.

Dreyer, Gunda: Verhaltenskodizes im Referentenentwurf eines Ersten Gesetzes zur Änderung des Gesetzes gegen unlauteren Wettbewerb - Wird das Wettbewerbsrecht zum Motor für die Durchsetzung vertraglicher Verpflichtungen?, WRP 2007, S. 1294-1303.

Emmerich, Volker: Unlauterer Wettbewerb, 8. Auflage, München, 2009.

Fenner, Christoph: Der Globale Pakt der Vereinten Nationen, Freiburg, 2004. 
Fezer, Karl-Heinæ: Lauterkeitsrecht, Kommentar zum Gesetz gegen den unlauteren Wettbewerb (UWG), Band 1, 2. Auflage, München, 2010.

- Lauterkeitsrecht, Kommentar zum Gesetz gegen den unlauteren Wettbewerb (UWG), Band 2, 2. Auflage, München, 2010.

Frenæ, Walter: Selbstverpflichtungen der Wirtschaft, Tübingen, 2001.

Fritzsche, Jörg: Anmerkungen zum BGH Urteil FSA-Kodex, LMK 2011, 316398.

Glöckner, Jochen: Der gegenständliche Anwendungsbereich des Lauterkeitsrechts nach der UWG-Novelle 2008 — ein Paradigmenwechsel mit Folgen, WRP 2009, S. 1175-1188.

Gloy, Wolfgang, Loschelder, Michael, Erdmann, Willi: Handbuch des Wettbewerbsrechts, 4. völlig neu bearbeitete Auflage, München, 2010.

Götting, Horst-Peter, Nordemann, Axel: UWG Handkommentar, 1. Auflage, BadenBaden, 2010.

Harte-Bavendamm, Henning, Henning-Bodenig, Frauke: Gesetz gegen den unlauteren Wettbewerb, Kommentar, 1. Nachdruck, München, 2004.

- Gesetz gegen den unlauteren Wettbewerb, Kommentar, 2. Auflage, München, 2009.

Henning-Bodewig, Frauke: Richtlinienvorschlag über unlautere Geschäftspraktiken und UWG, GRURInt 2004, S. 183-193.

- Die Richtlinie 2005/29/EG über unlautere Geschäftspraktiken, GRUR Int. 2005, S. 629-634.

Hobe, Stephan: Einführung in das Völkerrecht, 9. aktualisierte und erweiterte Auflage, Tübingen, 2008.

Hoeren, Thomas: Das neue UWG und dessen Auswirkungen auf den B2B-Bereich, WRP 2007, S. 789-794.

- Das neue UWG — der Regierungsentwurf im Überblick, BB 2008, S. 1182-1191.

Huismann, Wilfried: Schwarzbuch WWF — Dunkle Geschäfte im Zeichen des Pandas, München, 2012.

Igbanugo, Herbert A.: The Late Rev. Leon H Sullivan \& the Sullivan Principles: The Greatest Underrated Catalyst for Socio-Economic Development in SubSaharan Africa, abrufbar unter http:/ /www.igbanugolaw.com/resources / 133011_1.pdf, zuletzt abgerufen am 12.08.2013.

Institut für Europäisches Wirtschafts- und V erbraucherrecht e. V.: Studie zur Realisierbarkeit eines allgemeinen Rechtsrahmens zur Lauterkeit im Handelsverkehr, abrufbar unter http:/ / ec.europa.eu/consumers/rights/studies_reports_ en.print.htm\#studies, zuletzt abgerufen am 12.08.2013, zitiert als MicklitzStudie. 
Keßler, Jürgen, Micklitz, Hans-Wolfgang: Die Richtlinie 2005/29/EG über unlautere Geschäftspraktiken im binnenmarktinternen Geschäftsverkehr zwischen Unternehmern und Verbrauchern, Special zu Betriebsberater 2005, Beilage 13, S. 1-23.

Knauff, Matthias: Der Regelungsverbund: Recht und Soft Law im Mehrebenensystem, Tübingen, 2010.

Kocher, Eva: Unternehmerische Selbstverpflichtungen im Wettbewerb - Die Transformation von ,,soft law“ in ,hard law“ durch das Wettbewerbsrecht, GRUR 2005, S. 647-652.

Köhler, Helmut: „,Wettbewerbshandlung“ und „Geschäftspraktiken — Zur richtlinienkonformen Auslegung des Begriffs der Wettbewerbshandlung und zu seiner Definition im künftigen UWG, WRP 2007, S. 1393-1397.

- Vom deutschen zum europäischen Lauterkeitsrecht — Folgen der Richtlinie über unlautere Geschäftspraktiken für die Praxis, NJW 2008, S. 3032-3037.

- Die UWG-Novelle 2008, WRP 2009, S. 109-177.

Köhler, Helmut, Bornkamm, Joachim: Gesetz gegen den unlauteren Wettbewerb, Kommentar, 31. neu bearbeitete Auflage, München, 2013.

Köhler, Helmut, Lettl, Tobias: Das geltende europäische Lauterkeitsrecht, der Vorschlag fu"r eine EG-Richtlinie u ber unlautere Geschäftspraktiken und die UWG-Reform, WRP 2003, S. 1019-1057.

Köpke, Ronald, Röhr, Wolfgang: Codes of Conduct - Verhaltensnormen für Unternehmen und ihre Überwachung, Köln, 2003.

Kübler, Friedrich und Kübler, Johanna: Werbefreiheit nach Benetton, in: Festschrift für Peter Ulmer zum 70. Geburtstag am 2. Januar 2003, hrsg. v. Mathias Habersach, Peter Hommelhoff, Uwg Hüffer, Kartsten Schmid, De Gruyter Recht, 2003, S. 907-928.

Lamberti, Andrea, Wendel, A. Domenik: Verkäufe außerhalb von Vertriebsbindungssystemen - Bringt die UWG-Reform neue Handlungsmöglichkeiten gegen „Außenseiter“?, WRP 2009, S. 1479-1488.

Münchner Kommentar zum Lauterkeitsrecht: Band I, München, 2006.

— Band II, München, 2006.

Nemeczek, Heinrich: Kein lauterkeitsrechtlicher ,per-se“- Schutz bei Verstoß gegen Verhaltenskodex. GRUR 2011, S. 431-433.

Nordemann, Axel, Nordemann Jan Bernd, Nordemann-Schiffel, Anke: Wettbewerbsrecht, Markenrecht, 11. Auflage, Baden-Baden, 2012.

Peters, Anna: Wege aus der Krise - CSR als strategisches Rüstzeug für die Zukunft, 2009, abrufbar unter http:/ / www.bertelsmann-stiftung.de/cps/rde/ xchg/SID-8493BAF0-D1E80665/bst/hs.xsl/downloads.htm?sort= Titel\&sortorder $=$ asc\&chunk $=2 \&$ suchbegriff $=$ anna $\% 20$ peters $\% 20$ wege $\% 20$ aus $\% 20 \mathrm{der} \% 20 \mathrm{krise} \& s u c h r u b r i k$, zuletzt abgerufen am 16.08.2013. 
Penkert, Helge: Social Accountability 8000: Der Weg zu einer menschengerechten Weltsozialordnung? In: Das ethische in der Ökonomie, Festschrift für Hans G. Nutzinger, Hrsg. von Thomas Beschorner und Thomas Eger, Marburg 2005, S. 403-430.

Piper, Henning, Obly, Ansgar, Sosnitza, Olaf: Gesetz gegen den unlauteren Wettbewerb, Kommentar, 5. neu bearbeitete Auflage, München, 2010.

Puchinger, Yvonne: Irreführung durch Informationspflichtverletzung, Konstanz, 2010.

Röns, Benjamin: Verhaltenskodizes und die Mitbestimmung nach dem Betriebsverfassungsgesetz, München/Ravenburg, 2007.

Rosner, Christian: Marken- und wettbewerbsrechtlicher Schutz selektiver Vertriebsbindungssysteme, Frankfurt am Main, 2005.

Sack, Rolf: Die lückenfüllende Funktion der Generalklausel des \ 3 UWG, WRP 2005, S. 531-544.

- Internationales Lauterkeitsrecht nach der Rom-II-VO, WRP 2008, S. 845-865.

Scherer, Inge: Das Ende des Verdikts der ,gefühlsbetonten“ Werbung - Aufgabe der „Sachlichkeits“-Doktrin?, GRUR 2008, S. 490-495.

- Die „Verbrauchergeneralklausel“ des $\int 3$ II 1 UWG - eine überflüssige Norm, WRP 2010, S. 586-592.

Scherrer, Christoph, Greven, Thomas: Instrumente zur Durchsetzung fundamentaler Arbeiterrechte, erschienen in: Andreas Georg Scherer, Karl-Hermann Blickle, Daniel Dietzfelbinger, Gerhard Hütter (Hg.), Globalisierung und Sozialstandards, Deutsches Netzwerk Wirtschaftsethik, dnwe Schriftenreihe Folge 9, München, 2002, Rainer Hampp Verlag, S. 163-178.

Schmidhuber, Martin: Verhaltenskodizes im nationalen und grenzüberschreitenden elektronischen Geschäftsverkehr — Zur Frage der Integration der Selbstregulierung durch Private in die staatliche Rechtsordnung, Frankfurt am Main, 2004.

- Verhaltenskodizes im neuen UWG — Überlegungen zur Bedeutung für die lauterkeitsrechtliche Praxis in Deutschland, WRP 2010, S. 593-599.

Schoenheit, Ingo, Bruns, Maren, Grünewald, Markus: Corporate Social Responsibility als Verbraucherinformation, imug Arbeitspapier 17/2007.

Schöttle, Hendrik: Die Schwarze Liste — Übersicht über die neuen Spezialtatbestände des Anhangs zu \ 3 Abs. 3 UWG, WRP 2009, S. 673-683.

Schulze, Reiner, Schulte-Nölke, Hans. Analysis of National Fairness Laws (Deutsche Übersetzung: Rechtsvergleichende Untersuchung des verbraucherschützenden Lauterkeitsrechts der Mitgliedstaaten), abrufbar unter http:/ / ec.europa. eu/consumers/rights/studies_reports_en.print.htm\#studies, zuletzt abgerufen am 12.08.2013, zitiert als Schulze/Schulte-Nölke. 
Senden, Linda: Soft Law in European Community Law, Portland, 2004.

Sosnitza, Olaf: Wettbewerbsbeschränkungen durch die Rechtsprechung — Erscheinungsformen und Ursachen auf dem Gebiet des Lauterkeitsrechts, Baden-Baden, 1995

- Der Gesetzentwurf zur Umsetzung der Richtlinie über unlautere Geschäftspraktiken, WRP 2008, S. 1014-1034.

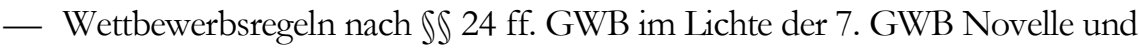
des neuen Lauterkeitsrechts, in: Recht und Wettbewerb, Festschrift für Rainer Bechtold, hrsg. v. Ingo Brinker, Dieter H. Scheuing, Kurt Stockmann, München 2006, S. 515-528.

Staudinger, Julius von: Kommentar zum Bürgerlichen Gesetzbuch mit Einführungsgesetz und Nebengesetzen, Band 2, Recht der Schuldverhältnisse, Neubearbeitung, 2009.

- Kommentar zum Bürgerlichen Gesetzbuch mit Einführungsgesetz und Nebengesetzen, Art. 1, 2, 50 - 218 EGBGB, Neubearbeitung, 2005.

Veelken, Winfried: Kundenfang gegenüber dem Verbraucher — Bemerkungen zum EG-Richtlinienentwurf über unlautere Geschäftspraktiken, WRP 2004, S. 1-30.

Walter, Axel von: Rechtsbruch als unlauteres Marktverhalten — Tatbestand und Anwendungsbereich des $\int 4$ Nr. 11 UWG in Abgrenzung zur Fallgruppe „Vorsprung durch Rechtsbruch“, Tübingen, 2007.

Walter, Robert: Soft Law aus rechtstheoretischer und verfassungsrechtlicher Sicht, in: Michael Lang/Josef Schuch/Claus Staringer (Hrsg.), Soft Law in der Praxis. Corporate Governance Codes, internationale Bilanzierungsregeln, Richtlinien und Rechtsauskünfte der Finanzverwaltung, Code of Conduct der EU, Kommentare der OECD. Beiträge anlässlich eines Symposiums zu Ehren Wolfgang Gassners, Wien 2005, S. 21-28.

Weidert, Stefan: In „Bio“ we trust: Werbung mit Genehmigungen, Gütesiegeln und anderen Qualitätskennzeichen, GRURPrax, 2010, S. 351-355.

Werner, Klaus, Weiss, Hans: Das neue Schwarzbuch Markenfirmen — Die Machenschaften der Weltkonzerne, Wien, 2006.

Wiebe, Andreas, Kodek, Georg E.: Kommentar zum UWG - Gesetz gegen den unlauteren Wettbewerb, Wien, 2009.

Wiebe, Andreas: Wettbewerbs- und zivilrechtliche Rahmenbedingungen der Vergabe und Verwendung von Gütezeichen, WRP 1993, S. 74-90.

Wiebe, Andreas: Die ,guten Sitten“ im Wettbewerb - eine europäische Regelungsaufgabe?, WRP 2002, S. 283-293.

- Umsetzung der Geschäftspraktikenrichtlinie und Perspektiven für eine UWG-Reform, JBl. 2007, S. 67-81. 
Willemsen, Alexander: Einführung und Inhaltskontrolle von Ethikrichtlinien, Freiburg, 2009.

Wiring, Roland: \5 UWG über irreführende geschäftliche Handlungen: Eine Norm, die Irreführt?, NJW 2010, S. 580-582.

Zimmer, Reingard: Soziale Mindeststandards und ihre Durchsetzungsmechanismen - Sicherung internationaler Mindeststandards durch Verhaltenskodizes, 2008. 
Verhaltenskodizes gehören mittlerweile - zumindest bei international tätigen Unternehmen der westlichen Welt - zum Standard. Aus diesem Grund haben die in das UWG eingefügten Regelungen zur Unlauterkeit von geschäftlichen Handlungen im Zusammenhang mit Verhaltenskodizes zu einer gewissen Aufregung in der Wirtschaft geführt. Dies gilt insbesondere für $\S 5$ Abs. 1 Satz 2 Nr. 6 UWG, welcher den Verstoß gegen Verhaltenskodizes als irreführende und damit in aller Regel unzulässige geschäftliche Handlung einordnet.

Droht nunmehr eine Sanktionierung der Nichteinhaltung der von den Unternehmen freiwillig abgegebenen und von diesen als rechtlich unverbindlich angesehenen Verhaltenskodizes durch das Lauterkeitsrecht und damit eine faktische Verpflichtung zu deren Einhaltung?

Die vorliegende Arbeit beantwortet nicht nur diese Frage, sondern untersucht vollumfänglich, welche Verhaltensweisen im Zusammenhang mit Verhaltenskodizes nach dem UWG unzulässig sind. 